\title{
New insights from the international registry for extracranial carotid artery aneurysms
}

Perspectives on etiology and follow-up

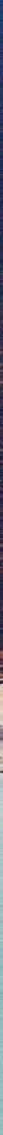

Constance J.H.C.M. van Laarhoven 

New insights from the international registry for extracranial carotid artery aneurysms

Perspectives on etiology and follow-up

Constance J.H.C.M. van Laarhoven 
New insights from the international registry for extracranial carotid artery aneurysms Perspectives on etiology and follow-up

PhD thesis, Utrecht University, The Netherlands.

(c) Constantia Josepha Henrina Christina Maria van Laarhoven, Utrecht 2020.

ISBN 978-94-6375-973-1

Cover Arie Onnink | Onnink Grafische Communicatie

Cover image "The Big Lagoon in the World Heritage listed Shark Bay region", by Sharkbaypixels (https://commons.wikimedia.org/wiki/File:The_Big_Lagoon_in_the_ World_Heritage_listed_Shark_Bay_region.jpg\#filelinks, licensed CC BY-SA 4.0 International)

Lay-out Wendy Schoneveld | www.wenzid.nl

Printing Ridderprint $\mid$ www.ridderprint.nl

Financial support by the Dutch Heart Foundation for the publication of this thesis is gratefully acknowledged.

Publication of this thesis was additionally supported and gratefully acknowledged by Boehringer Ingelheim, Chipsoft, Chirurgisch Fonds UMC Utrecht, Pie Medical Imaging, Stichting TWIN, and Terumo Aortic Nederland. 


\title{
New insights from the international registry for extracranial carotid artery aneurysms \\ Perspectives on etiology and follow-up
}

\author{
Nieuwe inzichten van de internationale registratie \\ voor aneurysmata van de extracraniële arteria carotis \\ Perspectieven op etiologie en follow-up \\ (met een samenvatting in het Nederlands)
}

\section{Proefschrift}

ter verkrijging van de graad van doctor aan de

Universiteit Utrecht

op gezag van de

rector magnificus, prof. dr. H.R.B.M. Kummeling,

ingevolge het besluit van het college voor promoties

in het openbaar te verdedigen op

donderdag 24 september 2020 des middags te 12.45 uur

door

Constantia Josepha Henrina Christina Maria van Laarhoven

geboren op 24 september 1991

te Tilburg 
Promotoren Prof. dr. G.J. de Borst

Prof. dr. D.P.V. de Kleijn 
Aan mijn familie 


\section{Contents}

Chapter 1 General introduction and thesis outline

\section{Part I | Contributing factors in aneurysmal disease}

Chapter 2 Delayed development of aneurysmal dilatations in patients with extracranial carotid artery dissections

\section{Submitted}

Chapter 3 Prevalence of extracranial carotid artery aneurysms in patients with intracranial aneurysms

PLoS One 2017

Chapter 4 Co-prevalence of extracranial carotid aneurysms differs between European intracranial aneurysm cohorts PLoS One 2020

Chapter 5 Systematic review of co-prevalence of arterial aneurysms within the vasculature

Eur J Vasc Endovasc Surg 2020

Chapter 6 Polygenic susceptibility of aortic aneurysms associates to the diameter of the aneurysm sac: the Aneurysm-Express biobank cohort

Sci Rep 2019

\section{Part II | Novel imaging techniques for follow-up}

Chapter 7 Volumetric assessment of extracranial carotid artery aneurysms Sci Rep 2019

Chapter 8 Gadolinium enhancement of the aneurysm wall in extracranial carotid artery aneurysms

Am J Neuroradiology 2020 
Chapter 9 Carotid tortuosity is associated with the presence of an extracranial carotid artery aneurysm

Submitted

\section{Part III | Discussion and summary}

Chapter 10 Summarizing discussion, future perspectives, and conclusions

Chapter 11 Summary in Dutch | Nederlandse samenvatting

Appendices Review Committee

Authors and affiliations

List of publications

Acknowledgements | Dankwoord

About the author 



\section{Chapter 1}

General introduction and thesis outline 


\section{General introduction}

The present thesis describes various aspects on how to improve healthcare for patients with aneurysmal disease of the extracranial carotid artery. The thesis mainly focusses on the identification of both clinical, and radiological risk factors that will benefit in allocating the risk for an adverse outcome, and subsequently indicate patients that will benefit from invasive therapy. In the following introduction, relevant background information and a brief outline of the thesis will be provided.

The word 'aneurysm' comes from the ancient Greek, and means dilatation or widening of an artery ${ }^{1}$. An aneurysm is a local outward bulging part of a blood vessel, caused by a weakened arterial wall. As the cellular composition of the arteries are more or less the same throughout the entire cardiovascular system, aneurysms can occur in any artery ${ }^{2}$. Literature indicates aneurysms are most prone to develop in locations where the vasculature bifurcates (e.g. circle of Willis ${ }^{3,4}$ ), or where impingement is prone to extensively test compliance and elasticity of the vessel wall (e.g. the infrarenal aorta), or a combination of both by ectatic vessels with less tone and sharpened (branched) angles ${ }^{2,5}$.

\section{Extracranial carotid artery aneurysm}

The extracranial carotid artery is a rare location for aneurysmal disease, and accounts for less than $1 \%$ of all peripheral artery aneurysms. Single-center reports estimated that up to $2.0 \%$ of all extracranial carotid interventions are due to an extracranial carotid artery aneurysm (ECAA $)^{6-9}$. The extracranial carotid artery includes the common carotid artery arising from the aortic arch and the brachiocephalic artery, the external carotid artery, and the internal carotid artery up to the skull base. The most observed locations for an ECAA are the carotid bifurcation, and within the distal part of the internal carotid artery ${ }^{10,11}$. Morphologically aneurysms are usually considered either fusiform (spindlelike) or saccular (blood blister-like) ${ }^{2}$ (Figure 1). The aneurysm is defined as a fusiform dilatation of at least 1.5 times the non-affected contralateral artery, and for focal saccular shaped ECAA any distension is accepted ${ }^{12,13}$. In case of bilateral dilatation, the diameter of the non-affected ipsilateral artery is used as a reference. The non-affected mean diameters of the common carotid artery range from $6.0 \mathrm{~mm}$ in women to $6.5 \mathrm{~mm}$ in men, and the internal carotid artery ranges from $4.5 \mathrm{~mm}$ and $6.0 \mathrm{~mm}$ respectively ${ }^{14}$. Maximum diameters between 4.0 and $60.0 \mathrm{~mm}$ of ECAA have been reported ${ }^{10,12,15}$. Various etiologies for ECAA have been proposed including atherosclerotic wall changes, spontaneous or traumatic artery dissection, infection (i.e. mycotic sources), connective tissue diseases (i.e. fibromuscular dysplasia, Ehlers-Danlos or Marfan syndrome) $)^{7-11,15}$, or patients with immunodeficiencies such as Behçet's disease ${ }^{16}$ or human immunodeficiency virus ${ }^{17}$. However, most often the true etiology of ECAA is unknown and left to the radiologist' interpretation. 


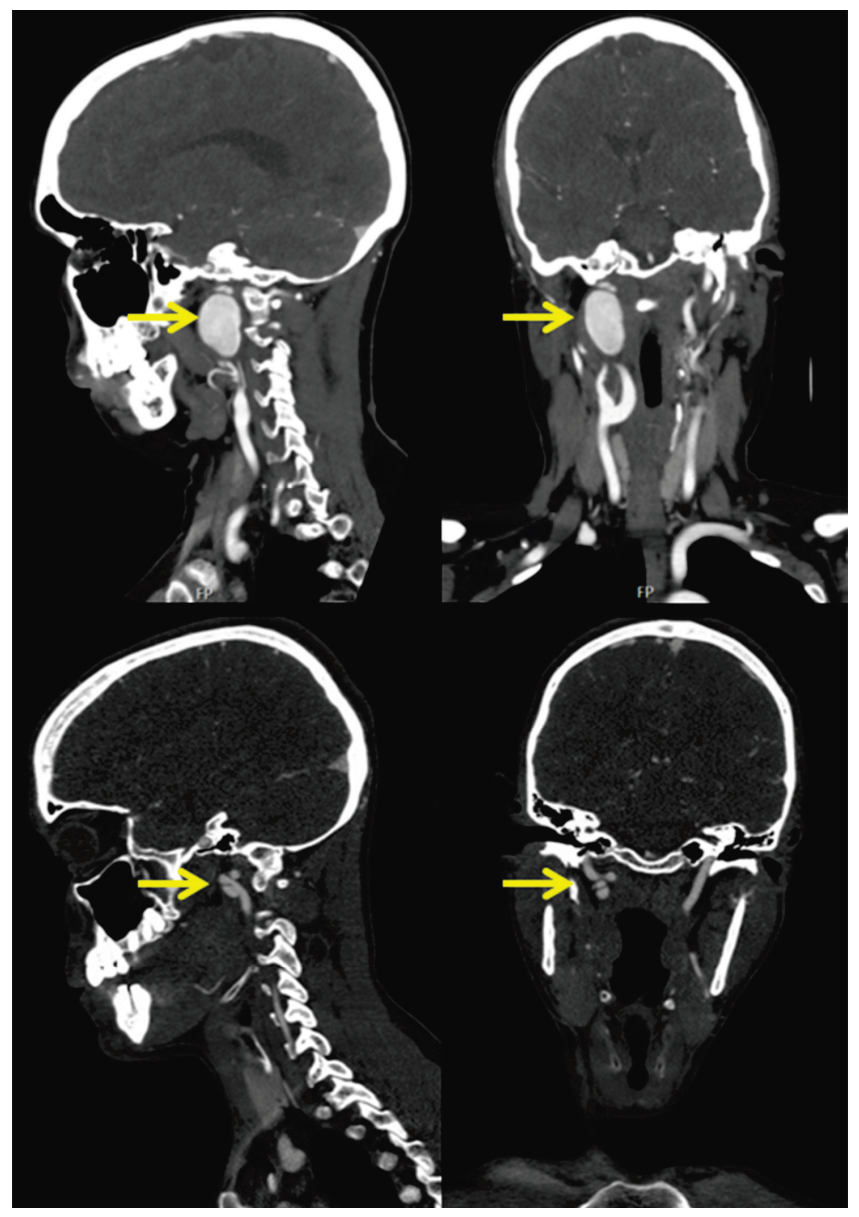

Figure 1. CT angiography of an ECAA, indicated by yellow arrows. Above: fusiform ECAA, and below: saccular ECAA, both of the right internal carotid artery. Reference: Carotid Aneurysm Registry.

\section{Clinical features}

The majority of ECAA patients is asymptomatic, and the carotid aneurysm is often found by coincidence ${ }^{12}$. The clinical presentation is dependent of the etiology, the location and the size of the carotid aneurysm ${ }^{13}$. If symptomatic, the most reported symptoms are cervical complaints like pain, feeling of a mass or thrill, and nerve palsies (such as Horner's syndrome) due to local compression by the dilated carotid artery. A smaller proportion of patients present with cerebral ischemia such as transient ischemic attack or stroke $\mathrm{e}^{6,9,12,15,18}$. The risk of rupture, a feared complication by affected patients, seems to be negligible in ECAA. In comparison with abdominal aortic aneurysm ${ }^{19}$ and stenotic carotid artery disease ${ }^{20}$, ECAA patients are younger and more often female. Patients with 
an ECAA have an average age of 55 years old at time of diagnosis ${ }^{12}$, and the male to female ratio is approximately 60:40 percent.

\section{Management}

The proposed reference standard to diagnose ECAA is by angiography, performed by either thin-slice computed tomography (CT) angiography or 3.0 Tesla magnetic resonance (MR) angiography. In addition to confirmation of the diagnosis, angiography images are used to classify the aneurysm in true or false, to monitor aneurysm growth and configuration, and to assess its extent and anatomy to explore potential treatment options ${ }^{21}$. Due to the rarity of ECAA, the natural clinical course and risk factors for adverse outcome are largely unknown ${ }^{18}$. As a consequence, evidence-based guidelines for the treatment of ECAA are non-existent. The goal of therapy is to reduce the risk of future (sub)clinical cerebral ischemia, or to prevent pharyngeal compression in large ECAAs $^{13}$. Invasive treatment ranges from traditional open aneurysm exclusion with or without interposition graft, to endovascular techniques, or a hybrid combination of both (Figure 2). Invasive exclusion of the aneurysm has been considered the treatment of choice for symptomatic or growing ECAA $^{12,13,18}$. The conservative approach (cardiovascular prevention medication and regular follow-up) may be appropriate in asymptomatic non-growing aneurysms, inoperable cases and patients with life-limiting comorbidities $^{12}$. The treatment of choice depends on the following clinical features; ECAA-related symptoms, presumed etiology, physical condition of the patient, location of the ECAA, and the well-being of the cerebrovascular tree. The majority of the existing data on the treatment of ECAA report the short-term outcome of traditional open surgery in symptomatic ECAA patients ${ }^{6,7,10,15,18}$. More insight in endovascular options, long-term outcome, and the natural course is essential to learn about the risk/benefit ratio of ECAA exclusion. In addition, the benefit of aneurysm exclusion in asymptomatic patients is

A

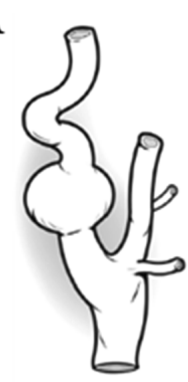

B

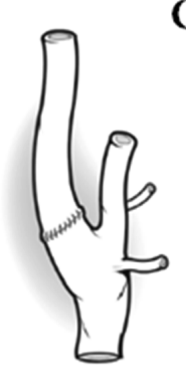

C

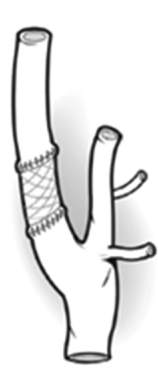

D

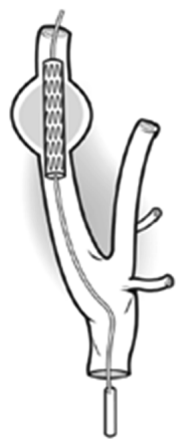

Figure 2. Schematic overview of invasive treatment. A: ECAA of the internal carotid artery, B: open surgical aneurysm exclusion without, and C: with interposition graft, and D: endovascular stenting of the ECAA. Reference: Carotid Aneurysm Registry. 
largely unexplored. It remains unclear whether asymptomatic ECAA patients are at risk of cerebral complications, and whether they should be exposed to the risks of invasive approaches. Besides treatment outcome, clinical and radiological markers need to be identified for well-considered ECAA-related risk determination.

\section{International Carotid Aneurysm Registry}

The first literature reporting an ECAA was by Sir Astley Cooper ${ }^{22}$, being one of the first surgeons to operate an aneurysm of the common carotid artery in 1805. Despite the knowledge of existence of ECAAs back then, our current insight in the natural course or optimized care for carotid aneurysms is still minimal in comparison with much more prevalent carotid stenotic disease. As a starting point to gain knowledge, the international Carotid Aneurysm Registry (www.carotidaneurysmregistry.com) was initiated in 2014 to register data of this seldom disease in a prospective manner ${ }^{23}$. The registry is based in the University Medical Center Utrecht, The Netherlands. Any patient with an ECAA is eligible for the ongoing inclusion of the registry, independent of etiology, symptomology, follow-up scheme or therapy. Besides demographic and aneurysm characteristics, the chosen treatment strategy and related clinical outcome are collected. Currently the registry includes 415 patients from more than 30 centers world-wide (dated April 2020), and is the largest available database of patients with an ECAA. Both short- and long-term follow-up of included patients is collected continuously, and future analyses of these data will form the base for clinical guidelines for ECAA. In addition, the registry cohort facilitated many sub studies described in the following thesis outline.

\section{Thesis outline}

In the present thesis we aimed to identify clinical features that are associated with ECAA, and investigated novel follow-up imaging techniques to monitor ECAA disease.

Part I focusses on identification of clinical risk features associated with ECAA. In chapter 2 independent risk features for time of onset of dissecting ECAA will be analyzed in a multi-center cohort of carotid artery dissection patients. As arterial aneurysms are known to have common clinical and genetic risk factors, we aimed to determine if ECAA are associated to other aneurysms of the cerebrovascular tree such as intracranial aneurysms. Chapter 3 describes the co-prevalence of ECAA in patients with an intracranial aneurysm from a large Dutch tertiary referral center, and in chapter 4 this cohort will be combined with another European cohort to analyze clinical risk features associated to ECAA in these patients. Insight in clinical risk profiles of more common aneurysms may be worthwhile, to understand co-prevalence of rare aneurysmal locations. In chapter 5 a systematic review of literature reporting on co-prevalence of arterial aneurysms in 
different vascular territories is described. Besides overlapping patient risk features among different aneurysms, future implementation of screening algorithms is discussed. In addition to clinical risk factors, several genome-wide association studies (GWAS) have been conducted to elucidate the strong heritability observed in particular abdominal aortic aneurysms. In chapter 6 a best-fit polygenic risk score based on previous studied GWAS estimates is modeled, and tested for association with clinical phenotypes. This study demonstrates the potential clinical utility of genetic data in the identification of high-risk patients in addition to established clinical risk factors in aneurysmal disease. Part II focusses on novel imaging techniques for monitoring ECAA disease. Due to the rarity of ECAA clinical practice is mainly extracted from the clinical guidelines for more common aneurysms. Consequently, the size of ECAAs is at present monitored by measuring the outer-outer bi-directional maximum diameter of the aneurysm sac. Chapter 7 introduces a protocol for volumetric assessment by use of CTA-based segmentations in order to monitor the various shapes and sizes of ECAA disease. This study is a step-up for future development of (semi-) automatic volumetric software. Another often used imaging strategy is MR, and in chapter $\mathbf{8}$ is the feasibility of detailed MR imaging of the aneurysm wall assessed. In a small explorative study, the relation of gadolinium-enhancement as potential surrogate marker for aneurysm growth and (sub) clinical cerebral complications is investigated. In chapter 9 carotid tortuosity will be assessed by use of different geometric values, and compared in a cross-sectional casecontrol study. The study shows that arterial tortuosity is bilateral fairly similar within ECAA patients, though distinctively higher in comparison with controls. Development of dedicated imaging techniques can contribute to risk stratification and future individualized medicine of ECAA patients. A summarizing discussion of all chapters including therefrom derived future perspectives and conclusions is provided in chapter 10. 


\section{References}

1. Wanhainen A, Verzini F, Herzeele I Van, et al. European Society for Vascular Surgery ( ESVS ) 2019 Clinical Practice Guidelines on the Management of Abdominal Aorto-iliac Artery Aneurysms. Eur J Vasc Endovasc Surg 2019.

2. Norman PE, Powell JT. Site specificity of aneurysmal disease. Circulation 2010;121:560-8.

3. Kanematsu Y, Kanematsu M, Kurihara C, et al. Critical roles of macrophages in the formation of intracranial aneurysm. Stroke 2011;42:173-8.

4. Sawyer DM, Pace LA, Pascale CL, et al. Lymphocytes influence intracranial aneurysm formation and rupture: Role of extracellular matrix remodeling and phenotypic modulation of vascular smooth muscle cells. $J$ Neuroinflammation 2016;13:1-9.

5. Dolan JM, Kolega J, Meng H. High wall shear stress and spatial gradients in vascular pathology: A review. Ann Biomed Eng 2013;41:1411-27.

6. McCollum C, Wheeler W, Noon G, et al. Aneurysms of the Extracranial Carotid Artery. Am J Surg 1979;137:196-200.

7. El-Sabrout R, Cooley DA. Extracranial carotid artery aneurysms: Texas Heart Institute experience. J Vasc Surg 2000;31:702-12.

8. Radak D, Davidovic L, Tanaskovic S, et al. A tailored approach to operative repair of extracranial carotid aneurysms based on anatomic types and kinks. Am J Surg 2014;208:235-42.

9. Donas KP, Schulte S, Pitoulias GA, et al. Surgical outcome of degenerative versus postreconstructive extracranial carotid artery aneurysms. J Vasc Surg 2009;49:93-8.

10. Nordanstig J, Gelin J, Jensen N, et al. National experience with extracranial carotid artery aneurysms: Epidemiology, surgical treatment strategy, and treatment outcome. Ann Vasc Surg 2014;28:882-6.

11. Attigah N, Külkens S, Zausig N, et al. Surgical Therapy of Extracranial Carotid Artery Aneurysms: Long-Term Results over a 24-Year Period. Eur J Vasc Endovasc Surg 2009;37:127-33.

12. Pourier VEC, Welleweerd JC, Kappelle LJ, et al. Experience of a single center in the conservative approach of 20 consecutive cases of asymptomatic extracranial carotid artery aneurysms. Eur J Neurol 2018;25:1285-9.

13. Pourier VEC, de Borst GJ. Which carotid artery aneurysms need to be treated (and how)? J Cardiovasc Surg (Torino) 2016;57:152-7.

14. Krejza J, Arkuszewski M, Kasner SE, et al. Carotid artery diameter in men and women and the relation to body and neck size. Stroke 2006;37:1103-5.

15. Garg K, Rockman CB, Lee V, et al. Presentation and management of carotid artery aneurysms and pseudoaneurysms. J Vasc Surg 2012;55:1618-22.

16. Lyazidi Y, Abissegue GY, Chtata HT, et al. Ruptured carotid aneurysm revealing a Behcet's disease. Ann Vasc Surg 2015;29:1317.e1-1317.e4.

17. Padayachy V, Robbs J V. Carotid artery aneurysms in patients with human immunodeficiency virus. J Vasc Surg 2012;55:331-7.

18. Welleweerd JC, Den Ruijter HM, Nelissen BGL, et al. Management of Extracranial Carotid Artery Aneurysm. Eur J Vasc Endovasc Surg 2015;50:141-7.

19. Lijftogt N, Karthaus EG, Vahl A, et al. Failure to Rescue e a Closer Look at Mortality Rates Has No Added Value for Hospital Comparisons but Is Useful for Team Quality Assessment in Abdominal Aortic Aneurysm Surgery in The Netherlands. 2018:652-61.

20. van Koeverden ID, van Haelst STW, Haitjema S, et al. Time-dependent trends in cardiovascular adverse events during follow-up after carotid or iliofemoral endarterectomy. Br J Surg 2017;104:1477-85.

21. Borst G de, Welleweerd J, Moll F. Aneurysms of the extracranial carotid artery. Second. (Oxford University Press, ed.). Oxford; 2016.

22. Cooper A. Account of the first successful operation performed on the common carotid artery for aneurysm in the year 1808 with the post-mortem examination in the year 1821. Guys Hosp Rep 1836.

23. Welleweerd JC, Bots ML, Kappelle LJ, et al. Rationale and design of the extracranial Carotid artery Aneurysm Registry (CAR). J Cardiovasc Surg (Torino) 2018;59:692-8. 



\title{
Chapter 2
}

\section{Delayed development of aneurysmal dilatations in patients with extracranial carotid artery dissections}

Submitted

\author{
C.J.H.C.M. van Laarhoven \\ M. Arnold \\ M. Danilova \\ M. Dreval \\ E. Ferrari \\ B. Goeggel Simonetti \\ J. Gralla \\ M. Heldner \\ L. Kalashnikova \\ M. Mancuso \\ T.M. Metso \\ V.K. Steinsiepe \\ D. Strbian \\ T. Tatlisumak \\ D.P.V. de Kleijn \\ G.J. de Borst
}




\section{Abstract}

\section{Background and purpose}

Dissection of the carotid artery (CaAD) may result in aneurysm formation. The present study was undertaken to evaluate time of onset of dissecting extracranial carotid artery aneurysms (ECAA) following CaAD, and analyze independent risk factors for development of these aneurysms.

\section{Methods}

From four European stroke centers, we included 360 patients with extracranial CaAD. Time of onset of dissecting aneurysms was analyzed, and putative clinical risk factors were assessed.

\section{Results}

Median follow-up was 5.2 months (range 0-24). A total of 75 dissecting ECAA were identified in 70 patients $(19.4 \%, 95 \%$ CI $15.7-23.8 \%)$. In 52/70 (74\%) patients, the ECAA was diagnosed at initial clinical work-up of CaAD diagnosis, median time 6 days after symptom onset (range 0-130). In the remaining eighteen patients (26\%) with multiple imaging upon ECAA detection, the median time was 6.2 months (189 days, range 8-426). Cox proportional hazards model showed that both multiple artery dissections $(2.78 \mathrm{HR}$, 95\% CI 1.65-4.68) and arterial tortuosity (1.68 HR, 95\% CI 1.02-2.79) were associated with presence of ipsilateral ECAA.

\section{Conclusion}

Approximately one out of five patients with CaAD developed an ipsilateral ECAA. The majority of ECAA was detected at initial vessel imaging. However in one fourth of CaAD patients, the dissecting ECAA was developed during follow up. Multiple dissections and arterial tortuosity were associated with the presence of dissecting ECAA at any time point. 


\section{Introduction}

Dissection of the cervical arteries (CeAD) is an important cause of stroke in young adults $^{1-3}$. It has been estimated that $13-49 \%$ of patients with CeAD will develop an ipsilateral dissecting aneurysm over time ${ }^{4-7}$, predominately in case of a carotid artery dissection $(\mathrm{CaAD})^{2}$. Previous multi-center data showed an increased number of extracranial carotid artery aneurysms (ECAA) at repeated follow-up imaging at three months after baseline, indicating that time of onset of ECAA varies among CaAD patients ${ }^{5}$. Although the clinical course of dissecting extracranial carotid artery aneurysms (ECAAs) is considered to be benign ${ }^{4,6,7}$, long-term follow-up imaging data in patients with $\mathrm{CaAD}$ are still limited. While asymptomatic ECAA are generally treated conservatively, surgical or endovascular ECAA exclusion may be considered in growing or symptomatic ECAA ${ }^{8}$. Better understanding of natural history of CaAD and thus ECAA pathophysiology and associated clinical risk factors is crucial in identification of patients at risk for adverse outcome. Since separate studies for dissecting ECAA after CaAD are sparse in literature, the present multicenter study was undertaken.

The objective of the present analysis was to evaluate time of onset of dissecting ECAA, and to identify independent risk factors associated with presence of these aneurysms.

\section{Methods}

\section{Participants}

Eligible patients were selected from four European stroke centers (Supplemental Table

I). These study centers were members of the Cervical Artery Dissection and Ischemic Stroke Patients (CADISP) consortium and the standard data collection was published before $^{2,4}$. Briefly, consecutive patients aged 18-years or older admitted to the Neurology department with suspect CeAD (both carotid and/or vertebral) were included and registered following a standardized protocol. The diagnosis of CeAD was defined as presence of a typical radiological aspect (intramural hematoma, aneurysmal dilatation, long tapering stenosis, intima flap, double lumen, or occlusion $>2 \mathrm{~cm}$ above the carotid bifurcation revealing an aneurysmal dilatation or a long tapering stenosis after recanalization) within the vascular wall of the internal carotid or vertebral artery ${ }^{9}$. Iatrogenic and purely intracranial artery dissections were excluded. For the present study, only patients with a spontaneous $\mathrm{CaAD}$ were included. Exclusion criteria were a traumatic, and/or purely vertebral artery dissection. All study procedures were approved by ethics committees at each participating center according to local legislation. 


\section{Clinical data}

Demographics and the following putative risk factors were recorded: hypertension (previously known, any antihypertensive treatment or blood pressure $\geq 140 / 90 \mathrm{~mm} \mathrm{Hg}$ on repeated measurement), diabetes mellitus (fasting glucose $>7 \mathrm{mmol} / \mathrm{L}$ or antidiabetic medication), any statin treatment, smoking (current, within 3 months or former usage), and migraine (classified according to International Headache Society criteria ${ }^{11}$ ). In addition, infection or any trauma within three months prior to the dissecting event, and known connective tissue disease were recorded by patient chart review. Presenting symptom (Horner's syndrome or cerebral ischemia) and time of symptom onset were recorded.

\section{Imaging data}

All available carotid imaging and reports were reviewed up to two years after dissection diagnosis. The following imaging characteristics were retrieved from the radiology report: time and type of imaging, arterial stenosis or occlusion, cerebral infarct on MRI, cervical arterial tortuosity in terms of coiling or kinking ${ }^{11}$, additional intracranial or multiple cervical artery dissections, and presence of aneurysmal dilatation. Used imaging modalities varied from CTA (64-slice), MRA (1.5 and 3.0T), DSA, and duplex ultrasonography of the carotids, or a combination of these modalities. An aneurysmal dilatation or dissecting ECAA was defined as $\geq 150 \%$ fusiform dilatation of the carotid artery in comparison with the non-affected contralateral side, or saccular aneurysms of any $\operatorname{size}^{12,13}$.

\section{Outcome and statistical analysis}

The primary outcome was time of diagnosis of dissecting ECAAs in the CaAD cohort. In addition, independent risk factors associated with dissecting ECAA were analysed. Time of diagnosis was assessed by calculating the time between reported CaAD onset, and first radiology report in which the dissecting aneurysm was described. Baseline characteristics of patients with or without dissecting ECAA were compared by use of Chi-squared test and independent T-test. Dissecting ECAA-free survival probability following $\mathrm{CaAD}$ was estimated using the Kaplan-Meier method; survival curves were compared using the log-rank test. Cox-proportional hazard regression analysis was used to identify predictors for development of a dissecting ECAA, and scaled Schoenfeld residuals were used to confirm the assumption of proportionality. Next to observed differences in baseline characteristics, sex and age were included in the model. $P<0.05$ was considered statistically significant throughout. Ties were corrected with use of the exact-method. All statistical analyses were conducted using SPSS v25.0 (IBM Corp. Released 2017. IBM SPSS Statistics for Windows, Version 25.0. Armonk, NY: IBM Corp.) and Rstudio v3.4.1 (RStudio Team (2016). RStudio: Integrated Development for R. RStudio, Inc., Boston, MA, www.rstudio.com). 


\section{Results}

In total $416 \mathrm{CeAD}$ patients were analysed from four stroke centers. After exclusion of patients with a traumatic dissection $(\mathrm{n}=30)$, and patients without any available imaging information $(\mathrm{n}=26), 360 \mathrm{CaAD}$ patients remained for analysis (Figure 1, Supplemental Table I,II). Approximately 60\% (214/360) of patients were men, and the mean age was $45.2 \pm 9.8$ years-old. Baseline characteristics of the patients are summarized in Table 1. Overall median follow-up was 5.2 months (range 0-24) with median of 2 follow-up scans (range 1-6).

\section{Time of onset of dissecting ECAA}

In total, 75 dissecting ECAAs were diagnosed in 70 patients (19.4\%, 95\% CI 15.7-23.8\%) of which 5 had bilateral ECAA. The majority of dissecting aneurysms were located at the distal internal carotid artery $(61 / 78,78 \%)$, and were saccular shaped $(51 / 78,65 \%)$. In $52 / 70(74 \%)$ patients, the ECAA was diagnosed at initial clinical work-up of CaAD diagnosis, median time 6 days (range 0-130). In the remaining 18 patients (26\%) with multiple imaging upon ECAA detection, the median time to diagnosis was 6.2 months (189 days, range 8-426), see Figure 2.

\section{Putative features for dissecting ECAAs}

Presence of multiple cervical artery dissections and tortuosity of the carotid arteries (Table 1) were more frequent in patients with dissecting ECAA. Current smoking status and an additional intracranial artery dissection were more frequent in patients without dissecting ECAA. The dissecting ECAA-free survival probability at 1-year follow-up after $\mathrm{CaAD}$ in patients with and without multiple artery dissections was $41 \%$ (95\% 25.4-67.3), versus $79.1 \%$ (95\% CI 72.5-86.4) respectively, log-rank test $p<0.001$ (Figure 3). Stratification on age $\geq 55$ years-old or sex (Supplemental Figure I) did not statistically differ in survival probability. After adjustment, presence of an additional intracranial artery dissection $(0.32 \mathrm{HR}, 95 \%$ CI $0.14-0.75)$ was negatively associated with presence of a dissecting ECAA (Table 2). The presence of multiple cervical artery dissections was strongly associated with dissecting ECAAs (2.78 HR, 95\% CI 1.65-4.68), and so was tortuosity of the cervical arteries (1.68 HR, 95\% CI 1.02-2.79).

\section{Discussion}

The present multicenter study of patients with $\mathrm{CaAD}$, showed that approximately one out of five CaAD patients developed an ipsilateral ECAA over time. One quarter of these dissecting ECAA was detected after initial clinical work-up. In this subset of patients 


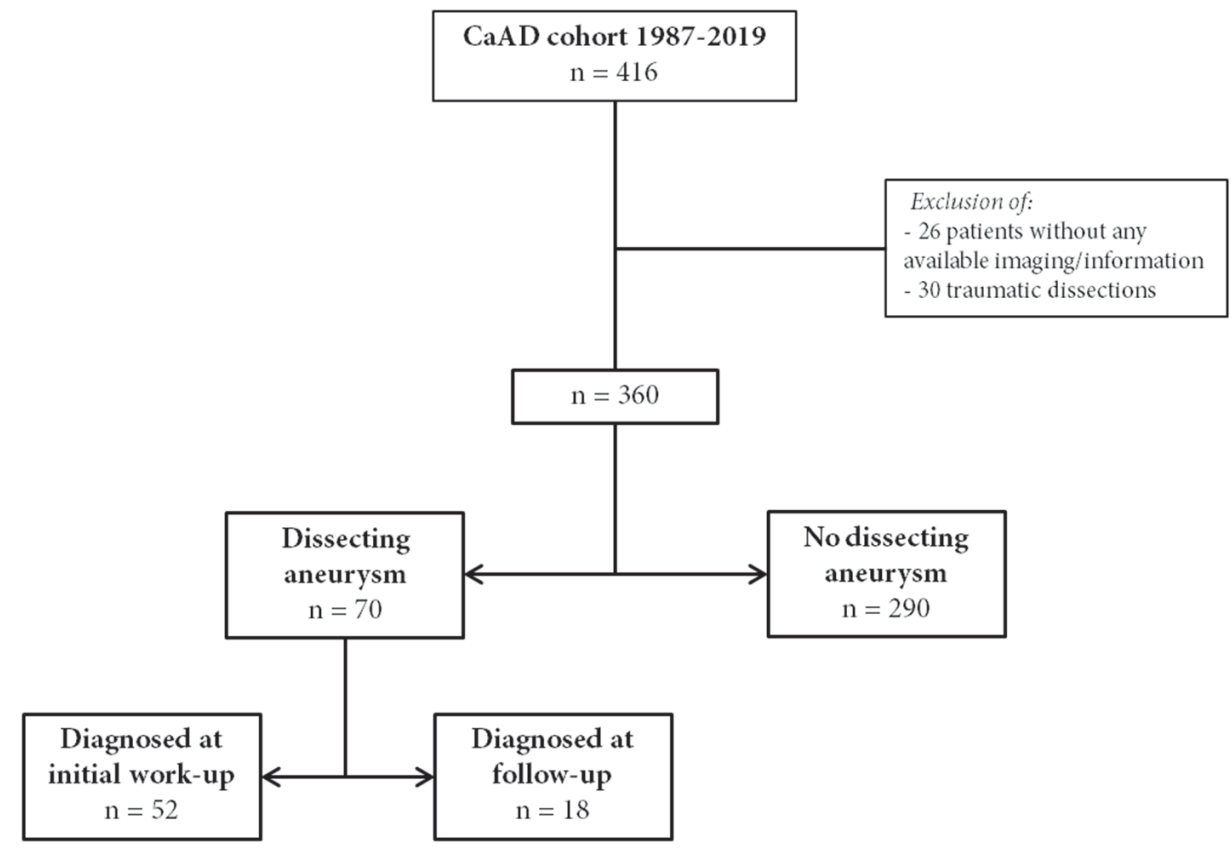

Figure 1. Flowchart of included carotid artery dissection (CaAD) study patients.

(18/70), median time to aneurysm diagnosis was six months after dissection symptom onset), though timing of ECAA diagnosis was highly subjective to applied follow-up scheme. Cox proportional hazard regression showed that multiple artery dissections and arterial tortuosity were associated with presence of dissecting ECAA at any time point. In contrast, an additional intracranial artery dissection seemed preventive for ECAA development.

Several studies have discussed characteristics and clinical outcome of dissecting aneurysms following $\mathrm{CeAD}^{4-7}$. Multi-center data indicated that time of onset could vary among dissecting aneurysm patients ${ }^{4}$. The results of the present study, in solely CaAD patients, also showed that identification of dissecting ECAA is highly dependent on applied follow-up interval. Although accurate estimation is not possible due to data heterogeneity, presumably one quarter of dissecting ECAA is developed and only diagnosed with follow-up imaging of the carotid arteries. Prolonged dynamic wall changes were also observed in a prospective MRA study of ten CaAD patients ${ }^{14}$, in which the mural hematoma in all participants was resolved at six months after symptom onset. Taken together this indicates $\mathrm{CaAD}$ is a dynamic process with both stenotic and aneurysmatic arterial wall changes in following days and months after dissection, and may require follow-up cerebrovascular imaging to detect potential subclinical ischemic 
Table 1. Baseline characteristics of 360 included CaAD patients.

\begin{tabular}{|c|c|c|c|c|c|}
\hline & \multicolumn{2}{|c|}{$\begin{array}{l}\text { CaAD without aneurysm } \\
\qquad n=290\end{array}$} & \multicolumn{2}{|c|}{$\begin{array}{l}\text { Dissecting aneurysm } \\
\qquad \mathbf{n}=70\end{array}$} & \multirow[t]{2}{*}{$p$-value } \\
\hline & $\mathrm{n}$ & $(\%)$ & $\mathrm{n}$ & $(\%)$ & \\
\hline Female sex & 120 & (41) & 26 & $(37)$ & 0.608 \\
\hline Age at admission (mean, sd) in years & 45.0 & 9.6 & 46.0 & 10.7 & 0.473 \\
\hline Hypertension & 100 & (34) & 27 & (39) & 0.642 \\
\hline Diabetes mellitus & 3 & (1) & 0 & $(0)$ & 0.897 \\
\hline Migraine & 80 & (28) & 23 & $(33)$ & 0.566 \\
\hline Connective tissue disease & 3 & (1) & 0 & $(0)$ & 0.905 \\
\hline Recent thrombosuction & 18 & (6) & 2 & (3) & 0.428 \\
\hline Recent trauma & 58 & (20) & 11 & $(16)$ & 0.462 \\
\hline Statin use & 21 & (7) & 4 & (6) & 0.862 \\
\hline Smoking history & 122 & (42) & 15 & $(21)$ & 0.001 \\
\hline Current & 88 & (30) & 11 & $(16)$ & 0.014 \\
\hline Former & 34 & (12) & 4 & (6) & 0.187 \\
\hline Multiple cervical artery dissection & 39 & (13) & 27 & $(39)$ & $<0.001$ \\
\hline Bilateral carotid & 16 & (6) & 19 & $(27)$ & \\
\hline Both carotid and vertebral & 23 & (8) & 8 & $(11)$ & \\
\hline Additional intracranial artery dissection & 80 & (28) & 6 & (9) & 0.001 \\
\hline Arterial tortuosity & 79 & (27) & 34 & $(49)$ & 0.001 \\
\hline Coiling & 62 & (22) & 24 & $(34)$ & 0.037 \\
\hline Kinking & 70 & (24) & 33 & $(47)$ & $<0.001$ \\
\hline Horner's syndrome & 98 & (34) & 30 & $(43)$ & 0.214 \\
\hline Cerebral ischemia & 47 & (17) & 6 & (9) & 0.141 \\
\hline
\end{tabular}

Bold indicates statistically significant. Abbreviations: CaAD: carotid artery dissection, sd: standard deviation.

events. Even though an exact estimation of the time of onset of dissecting ECAA is not feasible due to both patient and center burden in need of many imaging intervals in a short period of time, future studies should be performed with standardized follow-up intervals with imaging of the carotid arteries in any participating patient in order to define a more accurate time frame in which the dissecting aneurysm has developed. The clinical prognosis and outcome of dissecting ECAA has been studied previously ${ }^{4-7,15}$, and is beyond the scope of the current study. Despite the distinct characteristics per artery type, both carotid and vertebral ${ }^{4,6,7,15}$ or intracranial ${ }^{5}$ dissecting aneurysms were conjointly analyzed in these studies without indication of time to diagnosis of the dissecting aneurysm. Although the length of maximum follow-up differs among those included patients (range 3.5-109 months), it was estimated that $\pm 50 \%$ of developed aneurysms were relatively stable during follow-up, and the other half decreased in size or gradually resolved $^{4-7}$. A histopathological study conducted in thirteen symptomatic ECAA patients 


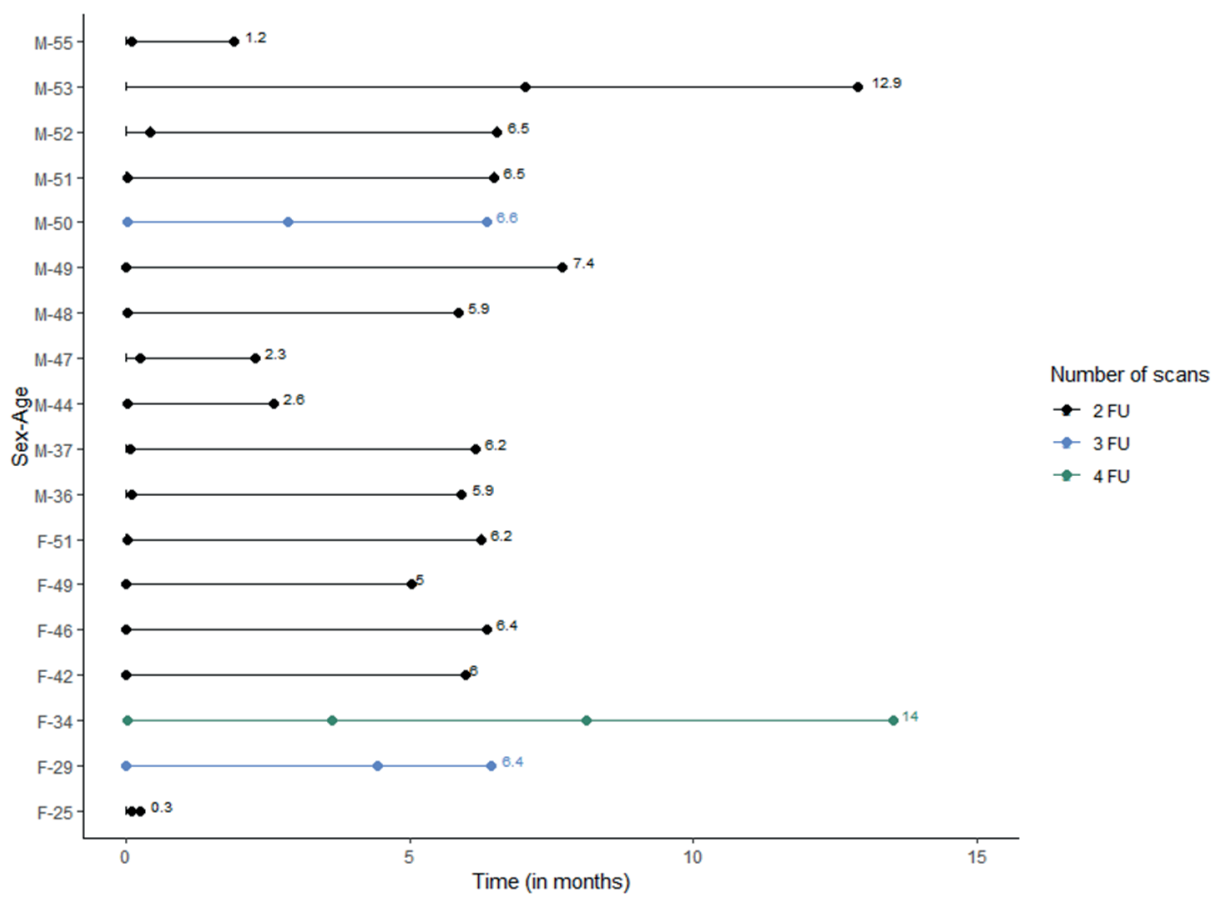

Figure 2. Reported time of detection (in months) of the dissecting ECAA after initial follow-up. On the x-axis is the time to detection of the dissecting ECAA given, and on the $y$-axis are the individual patients indicated. Dots represent imaging followup interval, last dot and value represents time of ECAA diagnosis. Abbreviations: M: male, F: female, FU: follow-up.

showed that the presumed etiology was $\mathrm{CaAD}$, despite the lack of radiological dissection signs such as intima flap at time of symptom presentation ${ }^{16}$. This indicates that even though most dissecting ECAA seem to have a benign course, some cases may cause symptoms during long-term follow-up. As local follow-up imaging schemes vary, or patients with mild CaAD symptoms refrain from further control visits, it is imaginable that additional ECAAs have been missed and diagnosed several years later. Putative features associated with ECAA growth and symptoms at any time-point remain to be identified. It is remarkable only three patients were diagnosed with a connective tissue disease, and none developed an ECAA. Available follow-up of these patients varied from 9 days, 6 and 19 months. Perhaps length of follow-up as well as the lack of genetic testing in regular clinical practice may explain the observed difference. Our finding that multiple artery dissections ${ }^{6,15,17}$ and arterial tortuosity ${ }^{6}$ are associated with dissecting ECAA, is in line with previous research. It seems reasonable that in case of multiple artery dissections, the vessel wall is at multiple sites locally weakened and thus more prone to aneurysm formation. Multiple artery dissection tended to cluster also in our cohort on the same artery type ${ }^{2}$, rather than involving both carotid and vertebral arteries (Table 1). In a 
Table 2. Cox proportional hazard regression analysis for dissecting aneurysms, available data $\mathrm{n}=331$ (of which 66 dissecting aneurysm patients).

\begin{tabular}{lllc}
\hline & HR & $\mathbf{9 5 \%} \mathbf{C I}$ & $\boldsymbol{p}$-value \\
\hline Female sex & 0.72 & {$[0.43-1.20]$} & 0.205 \\
\hline Age $\geq \mathbf{5 5}$ years-old & 0.99 & {$[0.51-1.91]$} & 0.977 \\
\hline Current smoker & 0.57 & {$[0.29-1.11]$} & 0.113 \\
\hline Multiple cervical artery dissection & 2.78 & {$[1.65-4.68]$} & $<\mathbf{0 . 0 0 1}$ \\
\hline Additional intracranial artery dissection & 0.32 & {$[0.14-0.75]$} & $\mathbf{0 . 0 0 8}$ \\
\hline Arterial tortuosity & 1.68 & {$[1.02-2.79]$} & $\mathbf{0 . 0 4 2}$ \\
\hline
\end{tabular}

Ties in survival data were corrected with the exact method. Bold indicates statistically significant. Abbreviations: HR: hazard ratio, CI: confidence interval.

previous large CADISP analysis $(n=1,958)$, multiple artery dissections were associated with increased risk of cerebral ischemic event at 3 to 6 months, and suggested as indicative of a transient vasculopathy ${ }^{18}$. Since $39 \%$ of all dissecting ECAA had multiple artery dissections (Table 1), these patients may also harbor increased risk of cerebral ischemic events especially during the first six months after $\mathrm{CaAD}$. The long-term cerebral outcome of the relatively young ECAA patients should be investigated in future longitudinal research. Additionally, arterial tortuosity has been described to be associated with CeAD ${ }^{19-21}$, and could reflect a separate vascular subtype which may be prone to exaggerated vascular remodeling. Moreover, arterial tortuosity reflects the weakness of the tunica media, which allows the intramural hematoma to spread towards the adventitial layer which may facilitate aneurysmal development. The preventive role of an additional intracranial dissection seems to be related to it leads to decreased intravascular and pulse pressure in the extracranial part of the internal carotid artery. Decreased pressure may reduce the potential for aneurysm formation.

\section{Limitations}

Some limitations need to be addressed while interpreting the results of the present study. This observational study was subject to locally applied follow-up practices. As a consequence, follow-up data imaging was not done as frequently as would have been needed to estimate time of onset accurately. In addition, potential ECAA cases may not have been identified as included patients may have had a relatively short follow-up period. Nevertheless, the present study is the largest study to date analyzing dissecting ECAA following $\mathrm{CaAD}^{4}$. Future standardized follow-up imaging studies at fixed time intervals to assess both vessel wall and brain outcome imaging are warranted. Second, indication for imaging was not systematically registered and therefore unknown. We anticipated on this by including only imaging up to two years after dissection onset, though indication bias is not completely ruled out. In addition, reliable quantitative measures and userfriendly software for CTA or MRA are available to assess arterial tortuosity ${ }^{22}$. 


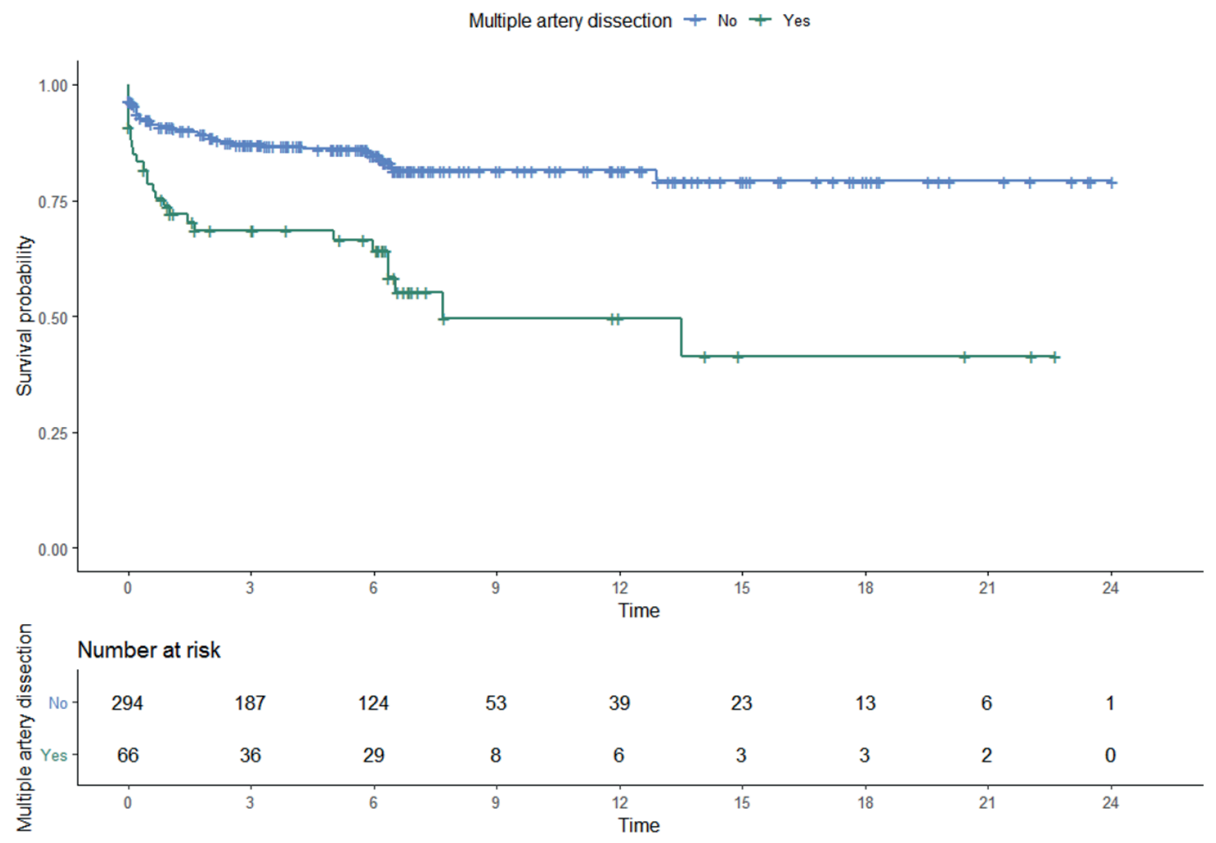

Figure 3. Kaplan-Meier curve and survival table for dissecting aneurysms, stratified on presence of multiple artery dissections (log rank test, $p<0.001$ ). Survival time is expressed in months, vertical annotation indicates censoring.

A quantitative tortuosity value incorporating in future prediction models could minimalize observer bias. Lastly, clinical and cerebral outcome of dissecting ECAA were beyond the scope of the present study. Future longitudinal studies including cerebral assessment are warranted to assess long-term cerebral outcome in these relatively young ECAA patients.

\section{Conclusions}

Within this multinational study of patients with CaAD, approximately one out of five patients developed an ipsilateral ECAA over time. Delayed development of dissecting ECAA was observed months after baseline, although diagnosis of ECAA was subjective to applied follow-up scheme. Multiple artery dissections and arterial tortuosity were associated with development of dissecting ECAA at any time point.

\section{Acknowledgements}

The authors thank the staff and participants of all participating centers for their important contributions. The study has been supported by the Swiss National Science Foundation (Grant 140340). 


\section{References}

1. Debette S, Leys D (2009) Cervical-artery dissections: predisposing factors, diagnosis, and outcome. Lancet Neurol 8:668-678.

2. Debette S, Grond-Ginsbach C, Bodenant M, et al (2011) Differential features of carotid and vertebral artery dissections The CADISP study. Neurology 77:1174-1181.

3. Christina A. Blum, Yaghi S (2015) Cervical Artery Dissection: A Review of the Epidemiology, Pathophysiology, Treatment, and Outcome. Arch Neurosci 2:e26670.

4. Larsson SC, King A, Madigan J, et al (2017) Prognosis of carotid dissecting aneurysms. Neurology 88:646-652.

5. Daou B, Hammer C, Chalouhi N, et al (2016) Dissecting pseudoaneurysms: Predictors of symptom occurrence, enlargement, clinical outcome, and treatment. J Neurosurg 125:936-942.

6. Touzé E, Randoux B, Méary E, et al (2001) Aneurysmal Forms of Cervical Artery Dissection, associated factors and outcome. Stroke 32:418-423

7. Guillon B, Brunereau L, Biousse V, et al (1999) Long-term follow-up of aneurysms developed during extracranial internal carotid artery dissection. Neurology 53:117-122.

8. Pourier VEC, de Borst GJ (2016) Which carotid artery aneurysms need to be treated (and how)? J Cardiovasc Surg (Torino) 57:152-157

9. Debette S, Metso TM, Pezzini A, et al (2009) CADISP-genetics: An International project searching for genetic risk factors of cervical artery dissections. Int J Stroke 4:224-230.

10. (2004) Headache Classification Subcommittee of the International Headache Society. The international classification of headache disorders: 2nd edition. Cephalalgia 9-160.

11. Zenteno M, Viñuela F, Moscote-Salazar LR, et al (2014) Clinical implications of internal carotid artery tortuosity, kinking and coiling: a systematic review. Rom Neurosurg 21:51-60.

12. Pourier VEC, Welleweerd JC, Kappelle LJ, et al (2018) Experience of a single center in the conservative approach of 20 consecutive cases of asymptomatic extracranial carotid artery aneurysms. Eur J Neurol 25:1285-1289.

13. van Laarhoven CJHCM, Pourier VEC, Lindgren AE, et al (2020) Co-prevalence of extracranial carotid aneurysms differs between European intracranial aneurysm cohorts. PLoS One 15:1-10.

14. Heldner MR, Nedelcheva M, Yan X, et al (2015) Dynamic changes of intramural hematoma in patients with acute spontaneous internal carotid artery dissection. Int J Stroke 10:887-892.

15. Hassan AE, Zacharatos H, Mohammad YM, et al (2013) Comparison of single versus multiple spontaneous extra and/or intracranial arterial dissection. J Stroke Cerebrovasc Dis 22:42-48.

16. Welleweerd JC, Nelissen BGL, Koole D, et al (2015) Histological analysis of extracranial carotid artery aneurysms. PLoS One 10:1-9.

17. Béjot Y, Aboa-Eboulé C, Debette S, et al (2014) Characteristics and outcomes of patients with multiple cervical artery dissection. Stroke 45:37-41.

18. Compter A, Schilling S, Vaineau CJ, et al (2018) Determinants and outcome of multiple and early recurrent cervical artery dissections. Neurology 91:e769-e780.

19. Saba L, Argiolas GM, Sumer S, et al (2015) Association between internal carotid artery dissection and arterial tortuosity. Neuroradiology 57:149-153.

20. Kim BJ, Yang E, Kim NY, et al (2016) Vascular Tortuosity May Be Associated with Cervical Artery Dissection. Stroke 47:2548-2552.

21. Giossi A, Mardighian D, Caria F, et al (2017) Arterial tortuosity in patients with spontaneous cervical artery dissection. Neuroradiology 59:571-575.

22. de Vries EE, Pourier VEC, van Laarhoven CJHCM, et al (2019) Comparability of semiautomatic tortuosity measurements in the carotid artery. Neuroradiology 61:147-153. 


\section{Supplemental Material}

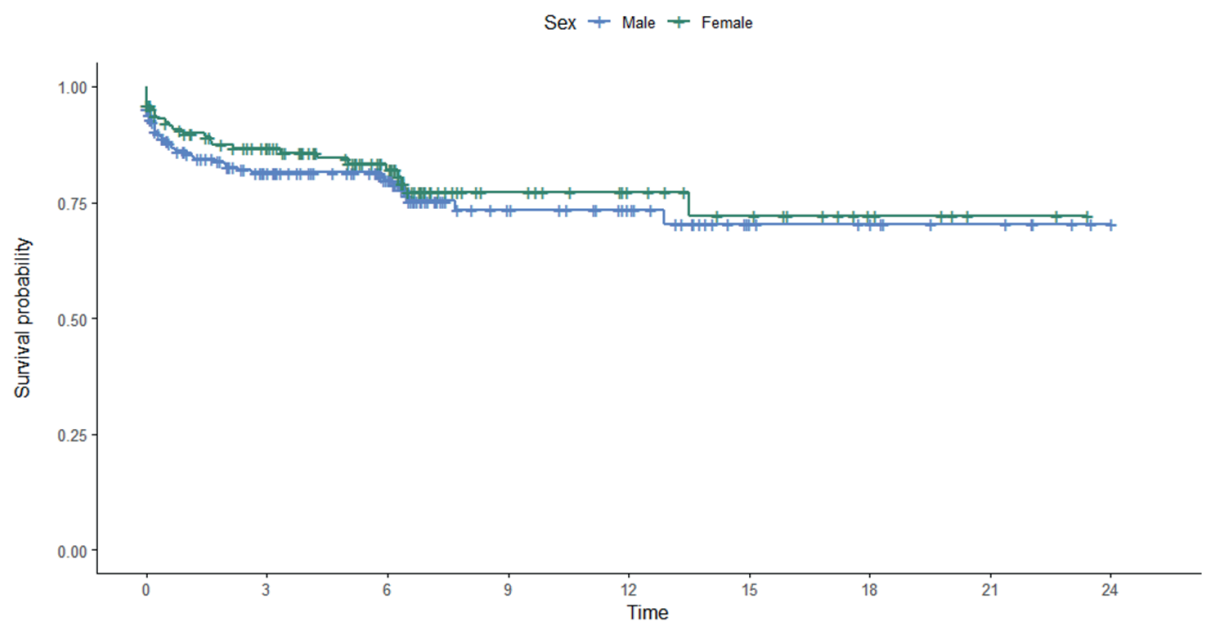

Number at risk
œ Male
Female

Supplemental Figure I. Kaplan-Meier curve and survival table for dissecting aneurysms, stratified per sex (log rank test, $p=0.5$ ). Survival time is expressed in months, vertical annotation indicates censoring.

Supplemental Table I. Participating centers and numbers of patients included per center.

\begin{tabular}{ccccc}
\hline & $\mathbf{n}$ & Hospital & City & Country \\
\hline 1. & 88 & Inselspital Bern & Bern & Switzerland \\
\hline 2. & 92 & Helsinki University Central Hospital & Helsinki & Finland \\
\hline 3. & 130 & Moscow University Hospital & Moscow & Russia \\
\hline 4. & 50 & University of Pisa & Pisa & Italy \\
\hline
\end{tabular}


Supplemental Table II. Patient characteristics per participating center.

\begin{tabular}{|c|c|c|c|c|c|c|c|c|}
\hline \multirow[b]{2}{*}{ Female sex } & \multicolumn{2}{|c|}{$\begin{array}{l}\text { Bern } \\
\mathrm{n}=88\end{array}$} & \multicolumn{2}{|c|}{$\begin{array}{l}\text { Helsinki } \\
\mathrm{n}=92\end{array}$} & \multicolumn{2}{|c|}{$\begin{array}{c}\text { Moscow } \\
n=130\end{array}$} & \multicolumn{2}{|c|}{$\begin{array}{l}\text { Pisa } \\
\mathrm{n}=50\end{array}$} \\
\hline & 36 & $(41)$ & 31 & $(34)$ & 63 & (49) & 16 & (32) \\
\hline $\begin{array}{l}\text { Age at admission } \\
\text { (mean, sd) in years }\end{array}$ & 47.9 & 10.3 & 47.2 & 10.2 & 40.5 & 8.0 & 49.1 & 8.2 \\
\hline Hypertension & 30 & $(35)$ & 41 & $(45)$ & 41 & $(32)$ & 15 & (30) \\
\hline Diabetes mellitus & 1 & (1) & 0 & - & 0 & - & 2 & (4) \\
\hline Migraine & 21 & $(27)$ & 35 & (38) & 38 & $(29)$ & 9 & (18) \\
\hline Connective tissue disease & 2 & (2) & 1 & (1) & 0 & $(0)$ & 0 & $(0)$ \\
\hline Recent thrombosuction & 15 & $(23)$ & 1 & (1) & 0 & $(0)$ & 1 & (2) \\
\hline Recent trauma & 8 & $(10)$ & 37 & $(40)$ & 17 & (13) & 7 & (14) \\
\hline Statin use & 7 & $(8)$ & 12 & (13) & 0 & $(0)$ & 6 & (12) \\
\hline Smoking history & 43 & (51) & 52 & (57) & 37 & (29) & 5 & (19) \\
\hline Current & 30 & (35) & 33 & (36) & 31 & (24) & 5 & (19) \\
\hline Former & 13 & (15) & 19 & (21) & 6 & (5) & 0 & - \\
\hline
\end{tabular}

Data are presented as $\mathrm{n}=$ number $(\%)$ unless otherwise indicated. 



\section{Chapter 3}

\section{Prevalence of extracranial carotid artery aneurysms in patients with an intracranial aneurysm}

PLoS One 2017;12:e0187479.

V.E.C. Pourier

C.J.H.C.M. van Laarhoven

M.D.I. Vergouwen

G.J.E. Rinkel

G.J. de Borst 


\section{Abstract}

\section{Background and purpose}

Aneurysms in various arterial beds have common risk- and genetic factors. Data on the correlation of extracranial carotid artery aneurysms (ECAA) with aneurysms in other vascular territories are lacking. We aimed to investigate the prevalence of ECAA in patients with an intracranial aneurysm (IA).

\section{Methods}

We used prospectively collected databases of consecutive patients registered at the University Medical Center Utrecht with an unruptured intracranial aneurysm (UIA) or aneurysmal Subarachnoid hemorrhage (SAH). The medical files of patients included in both databases were screened for availability of radiological reports, imaging of the brain and of the cervical carotid arteries. All available radiological images were then reviewed primarily for the presence of an ECAA and secondarily for an extradural/cavernous carotid or vertebral artery aneurysm. An ECAA was defined as a fusiform dilation $\geq 150 \%$ of the normal internal or common carotid artery or a saccular distention of any size.

\section{Results}

We screened 3,118 patient records (SAH database $n=2,186$, UIA database $n=932$ ), of which 2,931 had radiological images of the carotid arteries available. An ECAA was identified in $12 / 638$ patients (1.9\%; $95 \%$ CI 1.1-3.3) with completely imaged carotid arteries and in $15 / 2,293$ patients $(0.7 \%$; $95 \%$ CI $0.4-1.1)$ with partially depicted carotid arteries. Seven out of 27 patients had an additional extradural (cavernous or vertebral artery) aneurysm.

\section{Conclusion}

This comprehensive study suggests a prevalence for ECAA of approximately $2 \%$ of patients with an IA. The rarity of the disease makes screening unnecessary so far. Future registry studies should study the factors associated with IA and ECAA to estimate the prevalence of ECAA in these young patients more accurately. 


\section{Introduction}

Extracranial carotid artery aneurysm (ECAA) is a rare and accounts for less than $1 \%$ of all peripheral artery aneurysms ${ }^{1-3}$. The extracranial carotid artery includes the common carotid artery, the external carotid artery and the internal carotid artery (ICA) till the skull base. ECAAs are mostly incidental findings, commonly asymptomatic, and often identified in the $\mathrm{ICA}^{4}$.

Aneurysms in general are known to have common risk- and genetic factors and cooccurrences have been described in other arterial beds ${ }^{5}$. Due to the rarity of ECAAs it is unknown what the incidence, prevalence, association with aneurysms in other vascular territories and best treatment approach is ${ }^{6}$. As far as we know, an analysis for correlation between ECAA and IA has not been systematically performed before. Datasets of patients with an intracranial aneurysm (IA) are available in our center. Therefore, we had the opportunity to investigate the prevalence of ECAA in patients with an IA.

\section{Methods}

\section{Patient selection}

We performed a retrospective study in two prospectively collected databases of consecutive patients with an IA admitted to or seen at the outpatient clinic of the Department of Neurology and Neurosurgery of the University Medical Center Utrecht, The Netherlands. Approval was obtained from the Institutional Research Ethics Board. Patients were included from 1978 to 2015 in these datasets. One database included consecutive patients with subarachnoid hemorrhage ( $\mathrm{SAH}$ ), and one with unruptured intracranial aneurysms (UIA). Duplicates between the datasets were removed (i.e. patients in the UIA database with a ruptured IA during follow up). Patients were also excluded if no IA was present, for example if SAH resulted from trauma, an arteriovenous malformation, dural fistula, dissection without an aneurysm or perimesencephalic hemorrhage.

Two authors (VP, CL) screened the medical records of all patients in both datasets for available radiological imaging. Then, all original imaging (computed tomography angiography (CTA), magnetic resonance angiography (MRA), digital subtraction angiography (DSA), or duplex ultrasound (DUS) was reviewed for the presence of an ECAA. The available CTA evaluation was on 64-section CT scanners and MRA by the use of 1.5Tesla or 3Tesla scanners. Disagreements were discussed with a third independent observer (GB) until a final agreement was reached. Imaged carotid arteries were categorized into completely or partially depicted. Completely imaged carotid arteries were defined as images starting from the aortic arch until and/or beyond the skull base, 
depicting both the common, internal as well as the external carotid artery. Carotid arteries were considered partially imaged when either side of the internal carotid arteries was completely depicted (i.e. DSA), or when only the distal carotid arteries were depicted until the second cervical vertebra (i.e. CTA Brain).

\section{Data collection}

The diagnosis of an ECAA was determined according to previous radiology reports and by reviewing the available imaging scans of each patient. An ECAA was defined as a dilation of the arterial diameter of $\geq 150 \%$ compared with the normal carotid artery diameter. The side (left versus right, or bilateral), site (common, internal or external carotid artery), shape (saccular, fusiform) and diameter of the aneurysm were retrieved from available reports and by evaluation of the available images by the authors (VP, CL) independently. In addition to the presence of an ECAA, data on other extracranial/ extradural cervical arterial aneurysms were collected and: age at presentation, sex, medical history (diabetes, cardiovascular disease, connective tissue disease, polycystic kidney disease), smoking history, medication and clinical presentation.

\section{Statistical analysis}

For continuous variables, we calculated means with standard deviations or medians with ranges. For categorical variables, absolute numbers and/or proportions were calculated with 95\% confidence intervals (CI). We calculated the proportion of patients with an ECAA in the complete and partially depicted carotid artery groups.

\section{Results}

\section{Patients and imaging}

After screening both the databases a total of 3,118 patients remained. Of these patients, 638 (20\%) had completely depicted and 2,293 (74\%) partially depicted carotid arteries (Figure 1). The remaining 187 patients (6\%) only had a CT or MRI of the brain without the extracranial cervical arteries being depicted. The available imaging modalities of the cervical arteries were CTA, MRA, DSA, and DUS (Table 1). Imaging of a patient with an ECAA is illustrated in figure 2.

An ECAA was identified in 12 of 638 patients (1.9\%; 95\% CI 1.1-3.3) with complete imaging of the carotid arteries, and in 15 of 2,293 patients $(0.7 \%$; $95 \%$ CI $0.4-1.1)$ with partial imaging. Seven of the 27 patients (26\%) with an ECAA had an additional extradural aneurysm (cavernous or vertebral artery). In 17 of the 27 (63\%) patients the IA and ECAA diagnosis was made simultaneously. In one patient the ECAA was diagnosed 3.7 years prior to the IA, in nine patients the IA was diagnosed prior to the ECAA because no imaging of the carotids was performed or available before. 


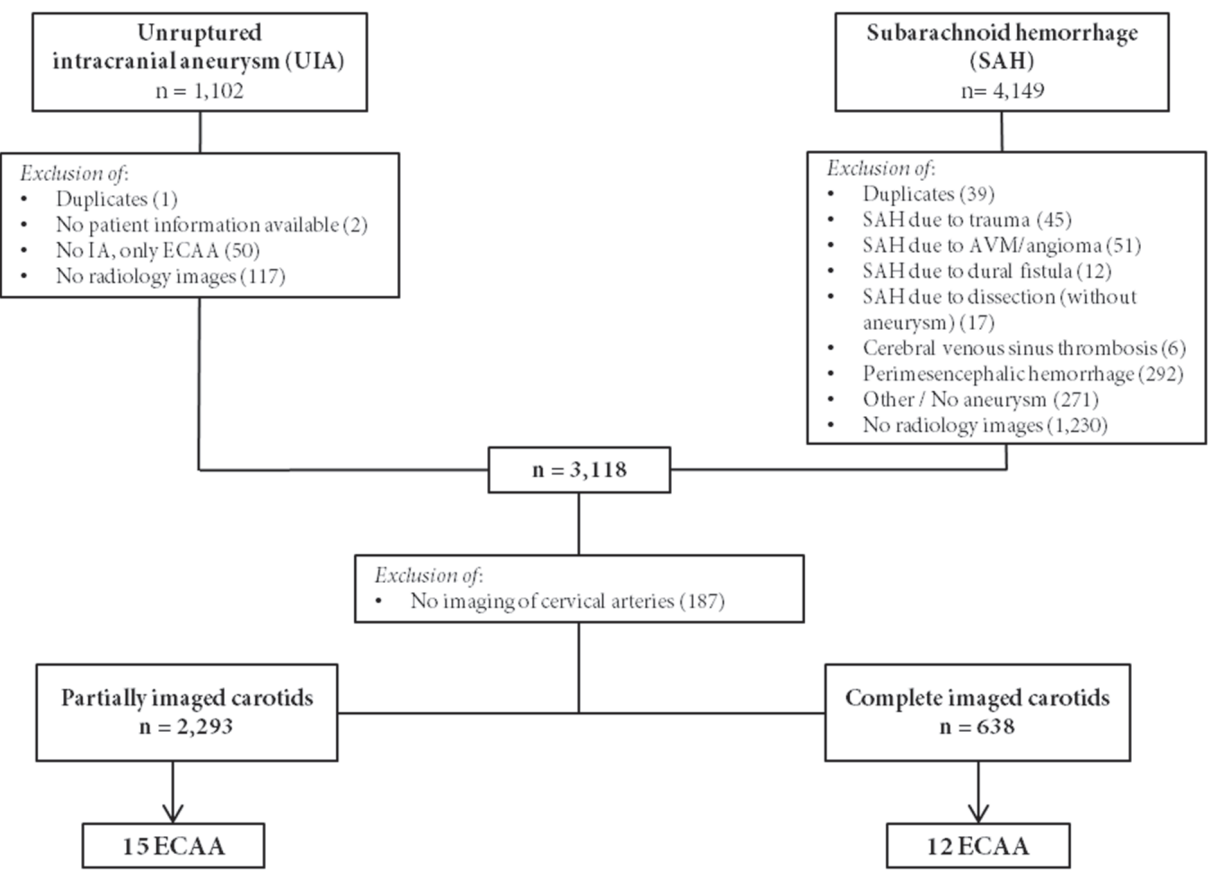

Figure 1. Flowchart of the 27 index patients with an IA and ECAA. Abbreviations: IA = intracranial aneurysm, ECAA = extracranial carotid artery aneurysm, UIA = unruptured intracranial aneurysm, $\mathrm{SAH}=$ subarachnoid hemorrhage, $\mathrm{AVM}=$ arteriovenous malformation.

\section{Patient characteristics}

Characteristics of the patients with an ECAA are presented in Table 2. The median age was 55 years (31-85). Fifteen patients (56\%) were women. Of all the patients with an ECAA, 24 (89\%) had no symptoms associated with the ECAA.

\section{Aneurysm characteristics}

Aneurysm characteristics are summarized in Table 3. All ECAAs were located in the internal carotid artery. The shape of the ECAA was saccular $(n=17)$ or fusiform $(n=17)$. The median size of the saccular ECAA was $8.0 \mathrm{~mm}$ (range 4-13 mm) and for fusiform ECAA $9.0 \mathrm{~mm}$ (range 6-12.5 mm). The etiology was mainly unknown, in five cases it was due to dissection and in one patient due to connective tissue disorder, namely EhlersDanlos type IV. 
Table 1. Available radiologic imaging modality of 3,118 patients.

n $(\%)$

\begin{tabular}{crl}
\hline Imaging of cervical arteries & & \\
\hline No angiography & 187 & $(6)$ \\
\hline Partially imaged carotid artery & 2293 & $(74)$ \\
\hline CTA brain & 1134 & $(36)$ \\
\hline MRA brain & 214 & $(7)$ \\
\hline DSA brain & 941 & $(30)$ \\
\hline DUS carotids & 4 & $(<1)$ \\
\hline Completely imaged carotid artery & 638 & $(20)$ \\
\hline CTA carotids & 310 & $(10)$ \\
\hline MRA carotids & 118 & $(4)$ \\
\hline DSA carotids & 18 & $(<1)$ \\
\hline DUS carotids & 190 & $(6)$ \\
\hline Intra- and extradural simultaneously & & \\
\hline ECAA prior to IA & 17 & $(63)$ \\
\hline IA prior to ECAA & 1 & $(4)$ \\
\hline Time, median (y) & 9 & $(33)$ \\
\hline Diagnosis time interval & 8.9 & $(3.4-23)$ \\
\hline
\end{tabular}

Data in $\mathrm{n}=$ number of patients (\%). Median, range (y) in years. Abbreviations: CTA = computed tomography angiography, MRA = magnetic resonance angiography, DSA = digital subtraction angiography, DSA= digital subtraction angiography, ECAA $=$ extracranial carotid artery aneurysm, IA = intracranial aneurysm.

\section{Discussion}

The present study shows that approximately $2 \%$ of the patients with an IA and completely imaged carotid arteries has an ECAA. No studies systematically investigated the prevalence of IA and ECAA before ${ }^{5}$. This prevalence could be an underestimation due to heterogeneity of the imaging modalities. In some patients DUS was used, but this modality is operator dependent and it cannot accurately detect distal extracranial carotid aneurysms ${ }^{7}$.

It remains unclear if IAs and ECAAs share the same etiology in the same patients. IAs are mostly saccular shaped ${ }^{8}$, while ECAAs have so far been described to be almost equally divided fusiform and saccular shaped ${ }^{4}$. Also, the wall structure of intracranial and extracranial vessels differs, which may indicate a different pathophysiology ${ }^{9}$. Contrary to IAs, atherosclerosis and dissection has been described as a main cause of ECAAs ${ }^{4,10-12}$. All identified ECAAs were located in the internal carotid artery; these would be classified as Attigah type $\mathrm{II}^{13}$. This is in accordance to our not yet published prospective clinical data from an ongoing web-based registry (www.carotidaneurysmregistry.com) $)^{12,14}$. 
Table 2. Characteristics of 27 patients with an ECAA.

Table 2. Characteristics of 27 patients with an ECAA.

\begin{tabular}{ccl}
\hline Age at ECAA diagnosis, median (range) & $\mathbf{n}$ & $\mathbf{( \% )}$ \\
\hline Women & 55 & $(31-85)$ \\
\hline Medical history & 15 & $(55.5)$ \\
\hline Diabetes & & \\
\hline$N R$ & 1 & $(4)$ \\
\hline Vascular & 2 & \\
\hline$N R$ & 12 & $(44)$ \\
\hline Cardiac & 1 & \\
\hline$N R$ & 5 & $(19)$ \\
\hline TIA/Ischemic stroke & 1 & \\
\hline$N R$ & 5 & $(19)$ \\
\hline Connective tissue disorder & 1 & \\
\hline$N R$ & 2 & $(7)$ \\
\hline Trauma in cervical region & 1 & \\
\hline$N R$ & 0 & $(0)$ \\
\hline Polycystic kidney disease & 1 & \\
\hline$N R$ & 1 & $(4)$ \\
\hline
\end{tabular}

Smoking history

$\begin{array}{ll}\text { Never } & 7 \\ \text { Quit smoking } & 7 \\ \text { Current smoker } & 9 \\ \text { Unknown } & 4\end{array}$

\section{Medication}

$\begin{array}{lrl}\text { None } & 12 & (44) \\ \text { Anti-hypertensive drugs } & 9 & (33) \\ \text { Anti-thrombotic drugs } & 5 & (19) \\ \quad \text { Mono-therapy } & 4 & (15) \\ \quad \text { Multiple-therapy } & 1 & (4) \\ \text { Lipid-lowering drugs } & 3 & (11) \\ \text { Unknown } & 3 & \end{array}$

\section{Family history}

$\begin{array}{lll}\text { Aneurysm } & 2 & (7) \\ \text { Cardiovascular } & 8 & (30) \\ \text { CTD } & 0 & \\ \text { PCKD } & 1 & (4) \\ \text { Unknown } & 8 & \end{array}$

\section{Clinical presentation}

$\begin{array}{lrl}\text { Asymptomatic } & 24 & (89) \\ \text { Ipsilateral ischemic stroke } & 1 & (4) \\ \text { Horner's syndrome } & 1 & (4) \\ \text { Local pain } & 1 & (4)\end{array}$

Number. of patients (\%) unless otherwise indicated. Median, (range) in years. Abbreviations: ECAA = extracranial carotid aneurysm. $\mathrm{NR}=$ not reported. $\mathrm{CTD}=$ connective tissue disease, $\mathrm{PCKD}=$ polycystic kidney disease 
Table 3. Aneurysm characteristics

\begin{tabular}{|c|c|c|c|c|}
\hline & IA, $n$ & (\%) & ECAA, $n$ & $(\%)$ \\
\hline Ruptured & 17 & (63) & 0 & \\
\hline \multicolumn{5}{|l|}{ Side } \\
\hline Left & 6 & (16) & 8 & $(30)$ \\
\hline Right & 10 & (26) & 12 & $(44)$ \\
\hline Bilateral & 11 & (58) & 7 & $(26)$ \\
\hline \multicolumn{5}{|l|}{ Site } \\
\hline CCA & & & 0 & \\
\hline ECA & & & 0 & \\
\hline ICA & & & 34 & $(100)$ \\
\hline Extradural cavernous carotid artery & & & $6^{\mathrm{b}}$ & \\
\hline Extracranial vertebral artery & & & $2^{\mathrm{b}}$ & \\
\hline \multicolumn{5}{|l|}{ Shape } \\
\hline Saccular & & & 17 & $(50)$ \\
\hline Fusiform & & & 17 & $(50)$ \\
\hline \multicolumn{5}{|l|}{ Size in $\mathbf{m m}$, median (range) } \\
\hline Saccular & & & 8.4 & $(4-13)$ \\
\hline Fusiform & & & 9.2 & $(6-12.5)$ \\
\hline \multicolumn{5}{|l|}{ Presumed etiology } \\
\hline Dissection & & & 5 & $(15)$ \\
\hline CTD & & & 2 & $(6)$ \\
\hline Not reported & & & 28 & (79) \\
\hline
\end{tabular}

Data in: $\mathrm{n}=$ number of aneurysms. ${ }^{\mathrm{b}}$ Patients with additional extradural cavernous carotid and vertebral artery aneurysm, not included in analysis.

Abbreviations: $\mathrm{IA}=$ intracranial aneurysm, $\mathrm{ECAA}=$ extracranial carotid aneurysm, $\mathrm{CCA}=$ common carotid artery, ECA

$=$ external carotid artery, ICA $=$ internal carotid artery, $\mathrm{CTD}=$ connective tissue disorder.

\section{Conclusion}

This is the first prevalence study of ECAA in patients with an IA. In this single-center, retrospective study we found a prevalence of $1.9 \%$. This prevalence indicates the possible rarity of the disease for which screening has not been indicated so far. However, the sparse knowledge on optimal work-up of ECAA is in contrast with the interest in management and long-term clinical outcome of relatively young patients, if left untreated. Future registry studies may elucidate the factors leading to co-existence of both IA and ECAA and estimate the prevalence of ECAA in patients with an IA more accurately. 


\section{References}

1. Welleweerd JC, den Ruijter HM, Nelissen BG, Bots ML, Kappelle LJ, Rinkel GJE, et al. Management of extracranial carotid aneurysm. Eur J Vasc Endovasc Surg 2015;50: 141-7.

2. Donas KP, Schulte S, Pitoulias GA, Siebertz S, Horsch S. Surgical outcome of degenerative versus postreconstructive extracranial aneurysms. J Vasc Surg 2009; 49:93-98.

3. de Borst GJ, Welleweerd JC, Moll FL. Aneurysms of the extracranial carotid artery. In: Thompson M, Boyle J, Brohi K, Cheshire N, Davies A et al. Oxford Textbook of Vascular Surgery, second edition. Oxford: Oxford University Press; 2016. p 462-67.

4. Welleweerd JC, Nelissen BG, Koele D, de Vries JP, Moll FL, Pasterkamp G, et al. Histological analysis of extracranial carotid artery aneurysms. PloS One 2015;10:e0117915.

5. Norman PE, Powel JT. Site specificity of aneurysmal disease. Circulation 2010; 121:560-68.

6. Pourier VEC, de Borst GJ. Which carotid artery aneurysms need to be treated (and how)? J Cardiovasc Surg (Torino) 2015.

7. Barlin K, Alexandrov AV. Vascular imaging in stroke, Neurotherapeutics. 2011; 8(3): 340-348.

8. Ruigrok YM, Elias R, Wijmenga C, Rinkel GJ. A comparison of genetic chromosomal loci for intracranial, thoracic aortic, and abdominal aortic aneurysms in search of common genetic risk factors. Cardiovasc Pathol $2008 ; 17: 40-47$.

9. Portanova A, Hakakian N, Mikulis DJ, Virmani R, Abdalla WM, Wasserman BA. Intracranial vasa vasorum: insights and implications of imaging. Radiology 2013; 235:667-79.

10. van Gijn J, Rinkel GJ. Subarachnoid hemorrhage: diagnosis, causes and management. Brain 2001; 124:249-78

11. van 't Hof FN, Ruigrok YM, Lee CH, Ripke S, Anderson G, de Andrade M, et al. Shared genetic risk factors of intracranial, abdominal and thoracic aneurysms. J Am Heart Assoc. 2016; 14;e002603.

12. Welleweerd JC, de Borst GJ. Extracranial carotid artery aneurysms: optimal treatment approach. Eur J Vasc Endovasc 2015; 49:235-6.

13. Attigah N, Kulkens S, Zausig N, Hansmann J, Ringleb P, Hakimi M, et al. Surgical Therapy of Extracranial Carotid Artery Aneurysms: Long-Term Results over a 24-Year Period. Eur J Vasc Endovasc Surg 2009; 37:127133

14. Welleweerd, JC, Bots ML, Kapelle LJ, Rinkel GJ, Ruigrok YM, Baas AF, et al. Rationale and design of the extracranial carotid artery aneurysm registry. J Cardiovasc Surg (Torino) 2015 2018;59: 692-698. 



\section{Chapter 4}

\section{Co-prevalence of extracranial carotid aneurysms differs \\ between European intracranial aneurysm cohorts}

PLoS One 2020,15:e0228041.

C.J.H.C.M. van Laarhoven

V.E.C. Pourier

A.E. Lindgren

M.D.I. Vergouwen

J.E. Jääskeläinen

G.J.E. Rinkel

G.J. de Borst 


\section{Abstract}

\section{Background and purpose}

Previously, we showed that co-prevalence of extracranial carotid artery aneurysms (ECAAs) in patients with intracranial aneurysms (IAs) was $2 \%$ in a Dutch cohort. In order to obtain more precise estimates and discover potential predictors of ECAA coprevalence in the European population, we retrospectively compared differences and similarities of our Dutch cohort with a Finnish cohort using protocolled imaging of the cerebrovascular tree.

\section{Methods}

IA patients within the prospective database of the Kuopio University Hospital were eligible for this study $(\mathrm{n}=1,118)$. Image analysis and hospital chart review were conducted.

\section{Results}

In total, 458 patients with complete carotid imaging conform protocol were analyzed. Twenty-four ECAAs in 21 patients were identified (4.6\%, 95\% CI 2.9-6.9), a higher coprevalence than in the Dutch cohort (1.9\%; 95\% CI 1.0-3.3), prevalence odds ratio (POR) 2.45 (95\% CI 1.19-5.03). In the Finnish cohort, $25 \%$ of all ECAAs were located around the carotid bifurcation, others in the internal carotid artery distally from the bifurcation. Independent predictors for ECAA co-prevalence were origin of country (POR 2.41, 95\% CI 1.15-5.06) and male sex (POR 2.25, 95\% CI 1.09-4.64).

\section{Conclusion}

The co-prevalence of ECAA in IA patients was twice as high in the Finnish compared to the Dutch IA cohort, with origin of country and male sex as independent predictors. Twenty-five percent of ECAAs would be missed, if the carotid bifurcation was not imaged. Therefore, we propose to always include imaging of the carotid bifurcation as the gold standard technique to identify ECAA in IA patients. 


\section{Introduction}

The extracranial carotid artery aneurysm (ECAA) is a rare vascular entity that accounts for less than $1 \%$ of all peripheral artery aneurysm $\mathrm{s}^{1-5}$. The majority of patients is asymptomatic, and the carotid aneurysm is often found by coincidence. If symptomatic, most observed symptoms are cervical complaints like pain, mass or thrill, and nerve palsies due to local compression by the dilated carotid artery. A smaller proportion of patients presents with cerebral ischemia, i.e. transient ischemic attack or stroke ${ }^{4-7}$. Due to the rarity of the disease, comprehensive literature and clinical guidelines are lacking. Since peripheral aneurysms share several risk and genetic factors, co-prevalence of arterial aneurysms within different vascular beds is commonly seen ${ }^{9-15}$. Although the evidence level for these findings are low and benefits of screening under debate, radiological screening for other (abdominal aortic) aneurysms may be indicated when patients are presented with a peripheral aneurys $\mathrm{m}^{16}$. In this light, our research group previously investigated co-prevalence of intracranial aneurysms (IAs) and ECAA in a selected Dutch IA cohort ${ }^{1}$. Due to the non-standardized and heterogeneous radiologic carotid imaging in IA patients, the reported co-prevalence of $\sim$ two percent of ECAA in IA patients, might be an underestimation.

In the present study, we studied another European IA cohort operating with a standard imaging protocol since 2007 including the aortic arch up to top of the brain by at least CTA or MRA. The primary aim was to compare the co-prevalence of ECAA in IA patients in the Finnish and Dutch cohort. Secondly, we combined the two cohorts to adjust for confounding and possibly identify independent predictors for ECAA presence.

\section{Methods}

\section{Patient population}

Following approval of the Institutional Research Ethics Board of the Kuopio University Hospital (KUH) for this retrospective study, we analyzed data from a prospective database (http://www.kuopioneurosurgery.fi/database) with patients admitted for IA to the KUH in Kuopio, Finland, from January 2010 to December 2016. All participants gave informed consent for this prospective database. All research was conducted according to the principles of the Declaration of Helsinki and its later amendments. The KUH is a tertiary referral center and the sole provider of neurosurgical services in its geographical catchment area (approximately 850,000 inhabitants) allowing prospective collection of a population-based IA cohort. Patients aged 18-years or older and with a radiologically confirmed IA were included. Exclusion criteria were: no extracranial carotid arterial 
imaging available, IAs caused by a trauma, arteriovenous malformation, cavernous malformation, dural fistula or cerebral venous sinus thrombosis. Crude data from a similar Dutch study performed in IA patients from the University Medical Center Utrecht (UMCU) were obtained (Supplemental Material II) ${ }^{1}$.

\section{Data collection}

All available cerebrovascular imaging was reviewed and categorized as complete or incomplete carotid imaging. Complete imaging of the cerebrovascular tree was defined as imaging from aortic arch up to top of the brain, depicting the common, internal, external and intracranial carotid arteries (i.e. CTA stroke or carotids). Imaging of the cerebrovascular tree was considered incomplete if imaging included only the distal (i.e. CTA Willis) part of the external and internal carotid artery (ICA), mostly from the second cervical vertebra up to the vertex. Patients undergoing CTA evaluation were scanned with 64-slice CT scanners, MRA imaging was performed on 1.5 or 3.0 Tesla scanners. Imaging was performed for a variety of reasons, including follow up of invasive or conservative treatment of the IA, stroke, dementia, or e.g. headache. All of the scans were reviewed on Sectra AB/PACS Software (Linköping, Sweden).

The diagnosis of IA was determined according to KUH IA database records. The diagnosis of ECAA was determined by reviewing the available radiological images of each patient and the original radiology reports. Fusiform or spindle-shaped ECAA was defined as $\geq 150 \%$ dilation of the arterial diameter, compared with the non-affected contralateral carotid artery diameter. In case of bilateral dilatation, the diameter of the non-affected part of the ipsilateral carotid artery was used as comparison. For saccular shaped ECAA, all sizes were accepted ${ }^{1,7}$. Location of ECAA was divided in proximal ECAA, around the carotid bifurcation, and distal ECAA. Proximal was defined as any location within the common carotid artery (Attigah type 5), and distal as any location in the internal carotid artery (Attigah type 1 and 2). If the ECAA location affected the carotid bifurcation, this was scored as around the carotid bifurcation (Attigah type 3 and 4) ${ }^{17}$. ECAA-related symptoms and presumed etiology of the ECAA were retrieved from the hospital records. The side (left or right), arterial site of the carotid artery, shape and diameter of ECAA were retrieved from available radiological reports. In case the size was not reported, two authors (CL, AL) independently measured the maximum aneurysm diameter on available examinations. Any disagreement in scoring was discussed with an experienced third and fourth independent observer (VP, GB) until final agreement was reached.

A hospital chart review was conducted for each patient to identify comorbidities and risk factors. Hypertension was defined as blood pressure of $\geq 140 / 90 \mathrm{mmHg}$ and/or use of antihypertensive medication, diabetes as any use of antidiabetic medication, hyperlipidemia as any use of blood lipid lowering drugs, cardiac disease was defined as any cardiac event (e.g. myocardial infarction, arrhythmia, or cardiac intervention like 
percutaneous coronary intervention), polycystic kidney disease was scored only if radiologically confirmed, and rheumatoid arthritis if stated within medical records. Smoking was defined as current smoking at time of ECAA diagnosis. Family history of IA was defined as $>1$ first degree relative with IA.

\section{Outcome and statistical analysis}

The primary outcome was defined as the co-prevalence of IA and ECAA in the Finnish cohort, as confirmed in the KUH radiology report. We compared the co-prevalence of the Finnish and Dutch cohort. Second, we combined the two cohorts with the aim to identify independent predictors for ECAA presence, analyzing only patients with complete carotid imaging, to ensure that the primary outcome is reliably measured. Differences were tested with Student two-tailed $t$ test and $\chi^{2}$ test when appropriate. For the primary analysis, the total number of ECAA patients was divided by the total amount of patients within the sample, and multiplied with 100 , and $95 \% \mathrm{CI}^{18}$ were calculated. The secondary outcome was assessed by multiple logistic regression. Potential confounders were selected based on a univariate analysis $(p<0.1)$ and literature ${ }^{1-6}$. Prevalence odds ratios (POR) and corresponding 95\% CI were calculated, and significance was set at $p<0.05^{19}$. All statistical analyses were performed using SPSS software version 25.0 for Windows (SPSS Inc, Chicago, Illinois, USA).

\section{Results}

In total, 1,70 IA patients were available from the KUH database. After exclusion of patients without available carotid imaging $(n=40)$, absence of intradural aneurysms ( $n$ $=8)$, AVM related IA $(\mathrm{n}=3)$ and one duplicate, 1,118 patients remained for analysis (see Figure 1). In 458 (41\%) patients, the cerebrovascular tree was completely imaged (CTA 91\%, MRA 9\%) conform protocol, while 660 (59\%) patients had incomplete carotid imaging.

\section{Comparison of Finnish and Dutch cohort}

In the total Finnish cohort, we identified 36 ECAAs in 33 Finnish IA patients (Figure 1). In the complete carotid imaging, 24 ECAAs in 21 patients were identified (Figure 2), which corresponds with a Finnish prevalence of $4.6 \%$ (21/458), 95\% CI 2.9-6.9. In the incomplete carotid imaging patients, only 12 patients with an ECAA were identified, resulting in a lower Finnish prevalence of 1.8\% (12/660), 95\% CI 0.9-3.2. Detailed ECAA characteristics are summarized in Supplemental Table I. In the Finnish cohort, all ECAAs were located in the ICA, divided in twenty-seven (75\%) distal ECAA, and nine (25\%) around the carotid bifurcation (Figure 3 ). 


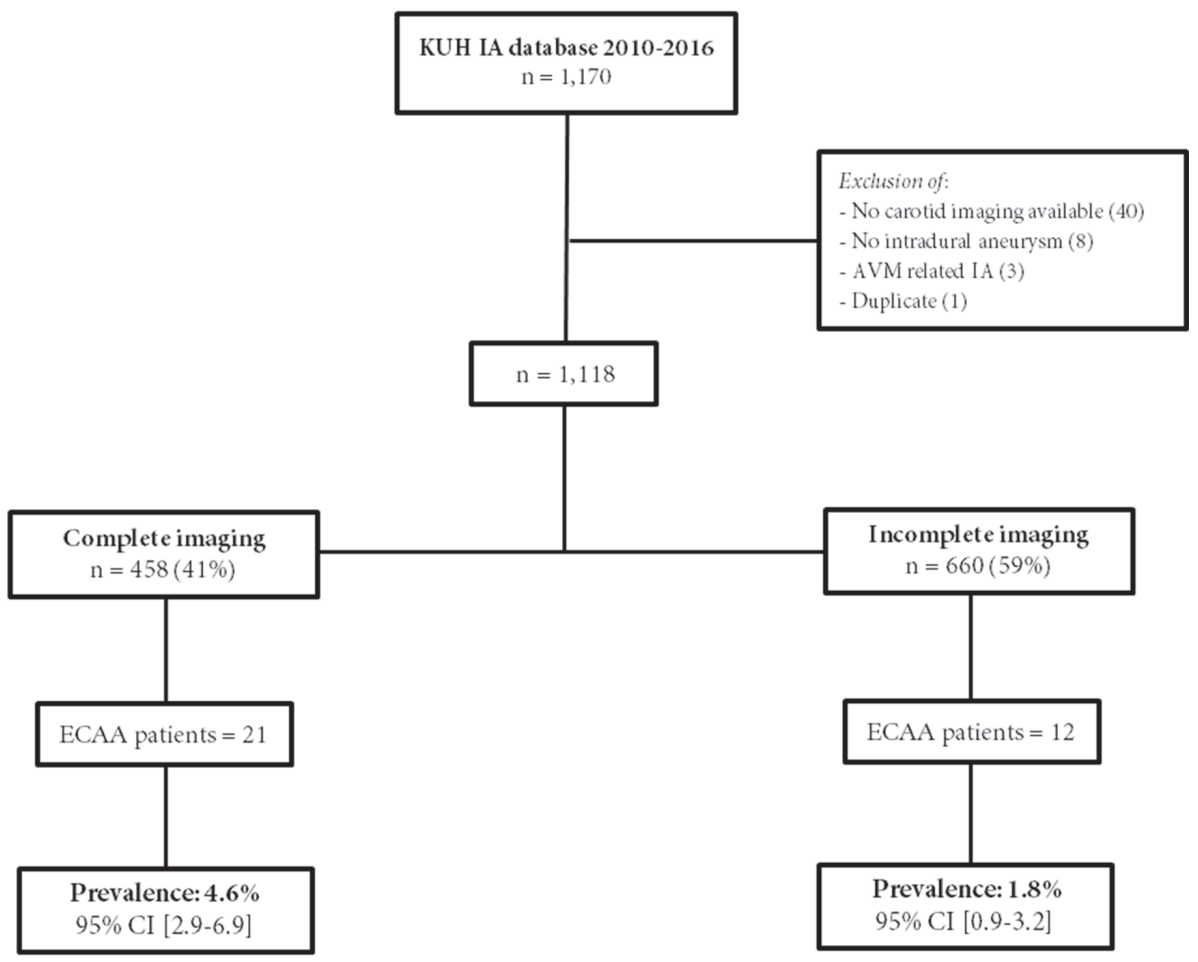

Figure 1. Flowchart of the Finnish cohort.

Abbreviations: $\mathrm{KUH}=$ Kuopio University Hospital, $\mathrm{IA}=$ intracranial aneurysm, $\mathrm{n}=$ number of patients, $\mathrm{AVM}=$ arteriovenous malformation, ECAA = extracranial carotid artery aneurysm.

The comparison of the co-prevalence of only the complete carotid imaging patients in the Finnish (4.6\%) and Dutch cohort (1.9\%), showed an unadjusted significant difference, $p=0.019$, POR $2.4595 \%$ CI 1.19-5.03. For further analysis, we only include patients with complete carotid imaging.

\section{Identification of clinical predictors}

The baseline characteristics of both the Dutch and Finnish cohort are summarized in Table 1. The cohorts differed in sex, age, hypertension, diabetes and statin use. In Table 2 , the univariate screen for factors associated with ECAA prevalence is shown. Besides sex $(p=0.021)$ and origin of cohort $(p=0.019)$, we included age, hypertension, diabetes and any statin use (Table 1) in the multiple logistic regression analysis. Both origin of country (POR 2.41, 95\% CI 1.15-5.06) and male sex (POR 2.25, 95\% CI 1.09-4.64) remained as independent factors for ECAA prevalence (Table 3). 


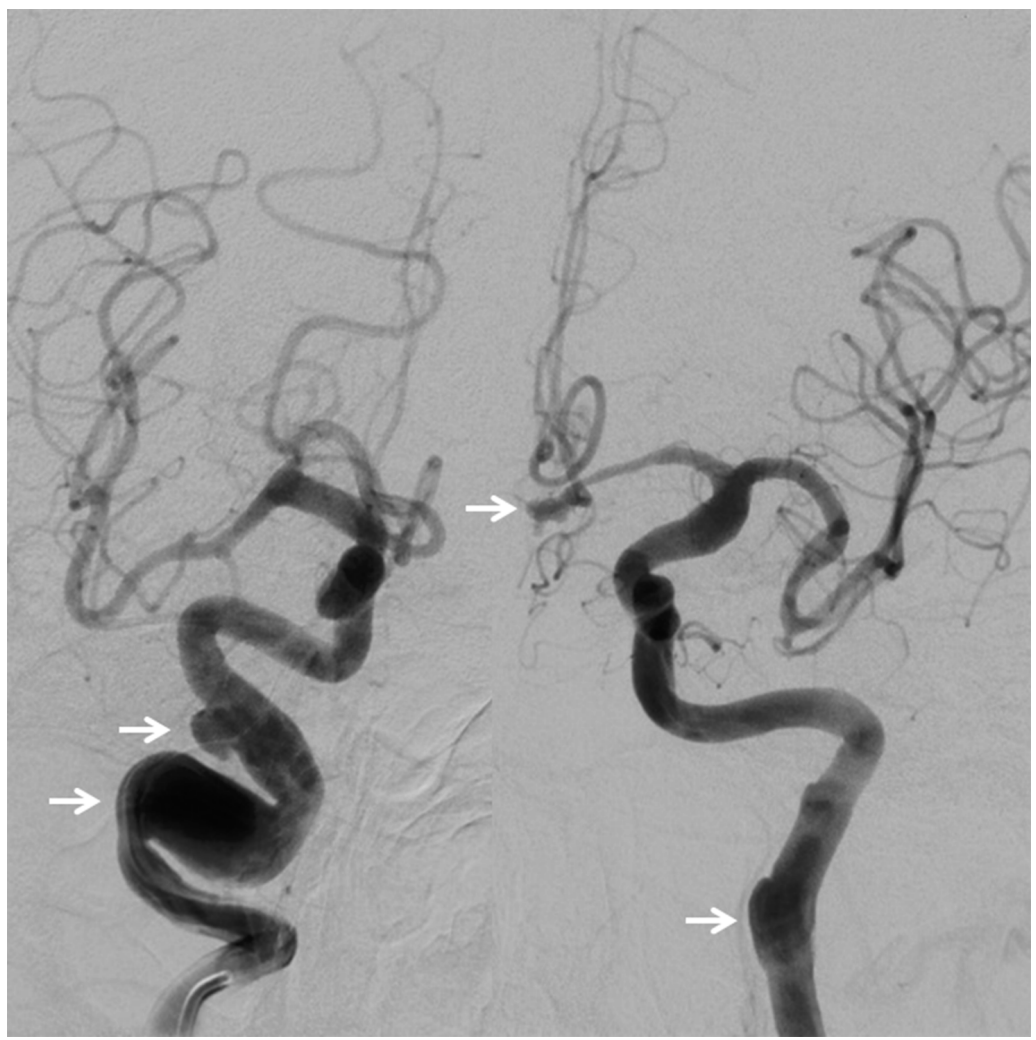

Figure 2. Overview DSA brain of an asymptomatic patient in posterior-anterior configuration.

Left: a fusiform ECAA in the right ICA and saccular shaped ECAA in the distal part of the RICA. Right: a fusiform dilated ECAA in the left ICA and a saccular shaped IA of the anterior communicating artery (all indicated by white arrows). Abbreviations: DSA = digital subtraction angiography, ECAA = extracranial carotid artery aneurysm, ICA = internal carotid artery, IA = intracranial aneurysm.

\section{Discussion}

The present study shows that approximately 1 out of 20 Finnish IA patients had an ECAA, which was more than twice the co-prevalence of the Dutch cohort. [1] After adjusting for confounding, origin of country and male sex remained as independent predictors for ECAA co-prevalence. Additionally, one out of four ECAAs in the Finnish cohort was located around the carotid bifurcation, and would have been missed if total carotid imaging was not performed (Figure 3).

Co-prevalence of aneurysms in different types of arteries has been widely reported in literature. Both co-existence of central abdominal aortic and thoracic aneurysms ${ }^{20,21}$, and peripheral aneurysms such as iliac, femoral or popliteal are described ${ }^{10-13}$. Over the years, evidence for a common pathway for both IA and aortic and/or thoracic aneurysms has 
been raised ${ }^{14,15,22,23}$. The co-prevalence of IA and ECAA does fit in this scientific field, although our present research does not provide clear answers why these aneurysms share co-existence. Age, size of aneurysm, current smoking ${ }^{14}$, hypertension ${ }^{15}$, and male sex ${ }^{12}$ were proposed as clinical predictors for aneurysm co-prevalence. In the present study, only male sex and Finnish origin remained as clinical predictors for ECAA co-prevalence. Differences in cardiovascular risk profile between our studied cohorts (Table 1) seem not to explain why the Finnish origin is associated with ECAA co-prevalence (Table 2), though might influence partially. Types of scanners were comparable in both cohorts, thus differences in imaging technologies have been ruled out. Although the number of patients that underwent arterial catheterization by DSA prior to the ECAA diagnosis was comparable in patients with IA and ECAA ( $42 \%$ Dutch vs $61 \%$ Finnish, $p=0.261$ ), iatrogenic dissections by the intraluminal intervention may have contributed to a higher rate of ECAAs in both cohorts. Most of iatrogenic pseudo-aneurysms have a benign course and tend to dissolve over time, therefore this influence is expected to be low. A genetic comparison in aneurysm related genes ${ }^{22,23}$ may elucidate why the Finnish origin

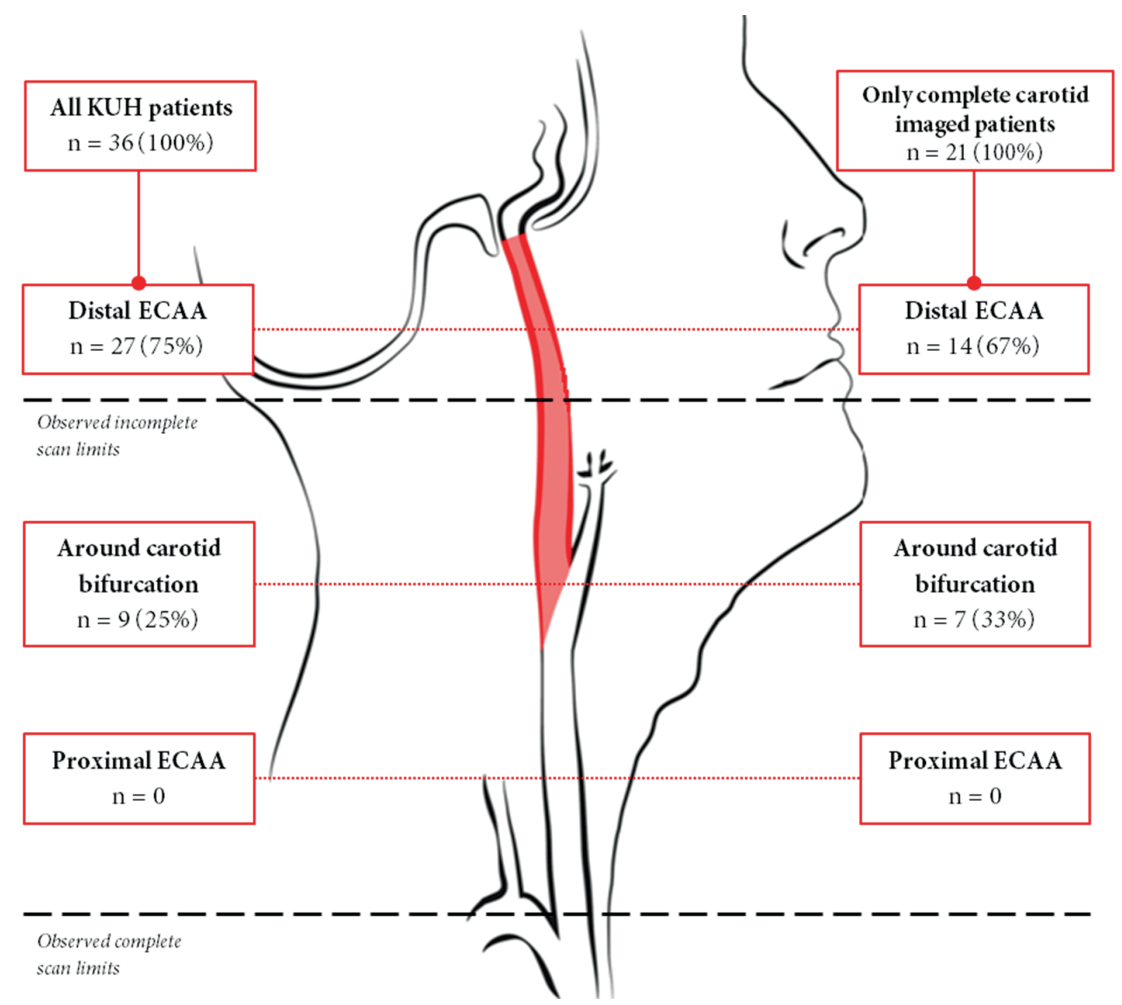

Figure 3. Schematic overview of location of 36 ECAAs in the extracranial carotid artery of the Finnish cohort. Abbreviations: $\mathrm{KUH}=$ Kuopio University Hospital, ECAA = extracranial carotid artery aneurysm. 
Table 1. Description of the study population with complete carotid imaging of the Finnish and Dutch cohort.

\begin{tabular}{lrrrrr}
\hline & $\begin{array}{c}\text { Finnish } \\
\mathbf{n}=\mathbf{4 5 8}\end{array}$ & $\begin{array}{c}\text { Dutch }^{\mathbf{a}} \\
\mathbf{n}=\mathbf{6 2 4}\end{array}$ & $\boldsymbol{p}$ \\
\hline Male sex & $\mathrm{n}$ & $(\%)$ & $\mathrm{n}$ & $(\%)$ & $0.001^{*}$ \\
\hline Age at admission (mean, sd) in years & 208 & $(45)$ & 222 & $(36)$ & $0.003^{*}$ \\
\hline Hypertension & 61 & 11.9 & 59 & 12.4 & $0.004^{*}$ \\
\hline Cardiac disease & 214 & $(49)$ & 241 & $(39)$ & 0.318 \\
\hline ADPKD & 90 & $(20)$ & 105 & $(17)$ & 0.461 \\
\hline Rheuma & 3 & $(1)$ & 8 & $(1)$ & 0.784 \\
\hline Diabetes & 12 & $(3)$ & 19 & $(3)$ & $0.000^{*}$ \\
\hline Family history IA & 57 & $(13)$ & 28 & $(5)$ & 0.935 \\
\hline Smoking, current & 27 & $(6)$ & 26 & $(6)$ & 0.175 \\
\hline Statin use & 141 & $(41)$ & 210 & $(37)$ & $0.000^{*}$ \\
\hline Presentation SAH & 216 & $(47)$ & 174 & $(29)$ & 0.196 \\
\hline
\end{tabular}

Abbreviations: $\mathrm{n}=$ number, $\mathrm{sd}=$ standard deviation, $\mathrm{ADPKD}=$ autosomal dominant polycystic kidney disease, $\mathrm{IA}=$ intracranial aneurysm, $\mathrm{SAH}=$ subarachnoid hemorrhage. ${ }^{*}$ indicates $p<0.05,{ }^{a}$ Crude data from Pourier et al. ${ }^{1}$

seems to be associated with higher ECAA co-prevalence, though obtaining a sufficient sample size would be challenging. In this way, the incidence of connective tissue disorders could be measured simultaneously.

The present study pointed out that if the carotid bifurcation is included within standard imaging protocol, almost every ECAA in an IA patient is detected (Figure 3). Hence, we propose to include imaging of the carotid bifurcation in IA patients as standard practice to identify ECAA in these patients (e.g. CTA stroke or carotids). In the Finnish cohort, $16 \%$ of patients faced ECAA-related symptoms like cerebral ischemia or nerve deficits (Supplemental Table I). As IA-related symptoms tend to be more dominant and permanent, this number might be underestimated. Although it is still unclear which ECAA-related symptoms an individual patient might encounter ${ }^{2,3,6-8}$, monitoring of growth or configuration of the ECAA is necessary to detect alterations and prevent potential cerebral ischemia in terms of (sub)clinical infarcts and white matter lesions, and the potential loss of the ipsilateral blood supply by the carotid artery. The largest ECAA review to date ${ }^{2}$ showed that $38 \%$ of treated ECAA patients were presented with stroke. This is of interest in patients who are already at high-risk of developing neurodegenerative diseases over-time. With a reasonable follow-up scheme, therapy decision making in IA and ECAA patients can be performed adequately. 
Table 2. Univariate screen for factors associated with ECAA prevalence.

\begin{tabular}{|c|c|c|c|c|c|}
\hline & \multicolumn{2}{|c|}{$\begin{array}{l}\text { ECAA } \\
\mathbf{n}=3^{\mathrm{a}}\end{array}$} & \multicolumn{2}{|c|}{$\begin{array}{c}\text { No ECAA } \\
\mathrm{n}=1049\end{array}$} & \multirow[t]{2}{*}{$p$} \\
\hline & $\mathrm{n}$ & $(\%)$ & $\mathrm{n}$ & $(\%)$ & \\
\hline $\begin{array}{l}\text { Age at admission } \\
\text { (mean, sd) in years }\end{array}$ & 59.7 & 12.2 & 56.9 & 14.3 & 0.198 \\
\hline Sex & & & & & $0.021^{*}$ \\
\hline Male & 20 & $(61)$ & 410 & (39) & \\
\hline Female & 13 & (39) & 639 & $(61)$ & \\
\hline Origin of cohort & & & & & $0.019^{*}$ \\
\hline Finland & 21 & $(64)$ & 437 & $(42)$ & \\
\hline The Netherlands & 12 & $(37)$ & 612 & $(58)$ & \\
\hline Hypertension & 16 & $(48)$ & 439 & $(42)$ & 0.665 \\
\hline Cardiac disease & 7 & $(21)$ & 188 & (18) & 0.828 \\
\hline ADPKD & 0 & - & 11 & (1) & - \\
\hline Rheuma & 2 & (6.1) & 29 & $(2.8)$ & 0.565 \\
\hline Diabetes & 2 & (6.1) & 83 & $(7.9)$ & 0.937 \\
\hline Family history IA & 1 & (3.0) & 52 & $(5.0)$ & 0.862 \\
\hline Smoking, current & 12 & $(36)$ & 339 & $(32)$ & 0.250 \\
\hline Statin use & 14 & $(42)$ & 376 & $(36)$ & 0.592 \\
\hline Prevalence of SAH & 12 & $(36)$ & 427 & $(41)$ & 0.749 \\
\hline
\end{tabular}

Abbreviations: $\mathrm{ECAA}=$ extracranial carotid artery aneurysm, $\mathrm{n}=$ number, $\mathrm{sd}=$ standard deviation, $\mathrm{ADPKD}=$ autosomal dominant polycystic kidney disease, $\mathrm{IA}=$ intracranial aneurysm, $\mathrm{SAH}=$ subarachnoid hemorrhage. ${ }^{*}$ indicates $p<0.05,{ }^{\mathrm{a}}$ Combined with crude data from Pourier et al. ${ }^{1}$

Table 3. Multiple logistic regression analysis for ECAA prevalence.

\begin{tabular}{lccc}
\hline Characteristics & $\begin{array}{c}\text { Complete case analysis } \\
\mathbf{n}=\mathbf{1 0 4 8}\end{array}$ & \\
\hline POR & {$[\mathbf{9 5 \%} \mathbf{~ C I}]$} & $\boldsymbol{p}$ \\
\hline Age & 0.98 & {$[0.95-1.01]$} & 0.105 \\
\hline Male sex & 2.25 & {$[1.09-4.64]$} & $0.028^{*}$ \\
\hline Origin of cohort & 2.41 & {$[1.15-5.06]$} & $0.020^{*}$ \\
\hline Hypertension & 1.41 & {$[0.67-2.99]$} & 0.368 \\
\hline Diabetes & 0.58 & {$[0.13-2.57]$} & 0.471 \\
\hline Statin use & 1.11 & {$[0.51-2.39]$} & 0.800 \\
\hline
\end{tabular}

Abbreviations: $\mathrm{ECAA}=$ extracranial carotid artery aneurysm, $\mathrm{n}=$ number, $\mathrm{POR}=$ prevalence odds ratio, $\mathrm{CI}=$ confidence interval. ${ }^{*}$ indicates $\mathrm{p}<0.05$, ${ }_{\text {a }}$ combined with crude data from Pourier et al. ${ }^{1}$ 


\section{Limitations}

Some limitations need to be addressed. First, although we used common definitions ${ }^{17}$ for proximal and distal carotid artery as well as around the carotid bifurcation, one might find this definition arbitrary. As our research had to anticipate on different scan limits on CTA/MRA despite the complete carotid artery imaging protocol, a breakdown of the carotid artery was necessary. Moreover, the indication for complete carotid imaging might have led to residual confounding in which we are unable to correct for in this retrospective study. A more precise estimate of the co-prevalence of IA and ECAA is obtained by scanning all IA patients with similar scan modality, limits and study period. Nevertheless, the present study reflects clinical practice in two large tertiary referral centers in Europe and is therefore highly applicable to IA standard care. Also, comorbidities were defined in a pragmatic way, since databases were combined. Although all CT scanners were 64-slice, thin-slice images were not always available for review due to local hospital storage. Small aneurysms $<3.0$ millimeter could have been missed and not included in our present analysis, but this is the case for both the Finnish and Dutch cohort. Lastly, due to the rarity of ECAA, a large sample size is not feasible. Hence, we performed our multiple logistic regression analysis conventionally despite our event rate. Potential overor under fitting of our model cannot be ruled out. Ideally, collaborations in the international ECAA registry (www.carotidaneurysmregistry.com) ${ }^{8}$ are needed to increase sample sizes and firmly indicate potential predictors for ECAA presence.

\section{Conclusion}

Co-prevalence of ECAA in IA patients was twice as high in another European cohort, with both origin of country and male sex as independent predictors. Approximately one out of four ECAAs would be missed if the carotid bifurcation was not imaged, hence we propose to always include imaging of the carotid bifurcation as the gold standard technique to identify ECAA in IA patients. 


\section{References}

1. Pourier VEC, van Laarhoven CJHCM, Vergouwen MDI, Rinkel GJE, de Borst GJ. Prevalence of extracranial carotid artery aneurysms in patients with an intracranial aneurysm. Plos One 2017;12:e0187479.

2. Welleweerd JC, Den Ruijter HM, Nelissen BGL, Bots ML, Kappelle LJ, Rinkel GJE, et al. Management of Extracranial Carotid Artery Aneurysm. Eur J Vasc Endovasc Surg. 2015;50: 141-147.

3. Donas KP, Schulte S, Pitoulias GA, Siebertz S, Horsch S. Surgical outcome of degenerative versus postreconstructive extracranial carotid artery aneurysms. J Vasc Surg. 2009;49: 93-98.

4. de Borst GJ, Welleweerd JC, Moll FL. Aneurysms of the extracranial carotid artery. Oxford Textbook of Vascular Surgery, second edition. Oxford: Oxford University Press; 2016. p 462-67.

5. Nordanstig J, Gelin J, Jensen N, Österberg K, Strömberg S. National experience with extracranial carotid artery aneurysms: Epidemiology, surgical treatment strategy, and treatment outcome. Ann Vasc Surg. 2014;28: 882-886.

6. Pourier VEC, De Borst GJ. Which carotid artery aneurysms need to be treated (and how)? J Cardiovasc Surg (Torino) 2016;57:152-157.

7. Pourier VEC, Welleweerd JC, Kappelle LJ, Rinkel GJE, Ruigrok YM, van der Worp HB, et al. Experience of a single center in the conservative approach of 20 consecutive cases of asymptomatic extracranial carotid artery aneurysms. Eur J Neurol. 2018;25: 1285-1289.

8. Welleweerd JC, Bots ML, Kappelle LJ, Rinkel GJ, Ruigrok YM, Baas AF, et al. Rationale and design of the extracranial Carotid artery Aneurysm Registry (CAR). J Cardiovasc Surg (Torino). 2018;59: 692-698.

9. Norman PE, Powell JT. Site specificity of aneurysmal disease. Circulation. 2010;121: 560-568.

10. Dawson I, Sie RB, van Bockel JH. Atherosclerotic popliteal aneurysm. Br J Surg. 1997;84:293-299.

11. Diwan A, Sarkar R, Stanley JC, Zelenock GB, Wakefield TW. Incidence of femoral and popliteal artery aneurysms in patients with abdominal aortic aneurysms. J Vasc Surg. 2000;31: 863-869.

12. Ravn H, Wanhainen A, Bjorck M. Risk of new aneurysms after surgery for popliteal artery aneurysm. $B r J$ Surg. 2008;95: 571-575.

13. Wallinder J, Georgiou A, Wanhainen A, Bjorck M. Prevalence of Synchronous and Metachronous Aneurysms in Women With Abdominal Aortic Aneurysm. Eur J Vasc Endovasc Surg. 2018.

14. Miyazawa N, Akiyama I, Yamagata Z. Risk factors for the association of intracranial and aortic aneurysms. Acta Neurochir(Wien). 2007;149: 221-229.

15. Kuzmik GA, Feldman M, Tranquilli M, Rizzo JA, Johnson M, Elefteriades JA. Concurrent Intracranial and Thoracic Aortic Aneurysms. Am J Cardiol. 2010;105: 417-420.

16. Wanhainen A, Verzini F, Herzeele I Van, Allaire E, Bown M, Cohnert T, et al. European Society for Vascular Surgery (ESVS) 2019 Clinical Practice Guidelines on the Management of Abdominal Aorto-iliac Artery Aneurysms. Eur J Vasc Endovasc Surg. 2019.

17. Attigah N, Külkens S, Zausig N, Hansmann J, Ringleb P, Hakimi M, et al. Surgical Therapy of Extracranial Carotid Artery Aneurysms: Long-Term Results over a 24-Year Period. Eur J Vasc Endovasc Surg. 2009;37: $127-133$.

18. Newcombe, RG. Two-Sided Confidence Intervals for the Single Proportion: Comparison of Seven Methods, Stat Med 1998;17:857-872.

19. Tamhane AR, Westfall AO, Burkholder GA, et al. Prevalence odds ratio versus prevalence ratio: choice comes with consequences. Stat Med, 2016;35:5730-5735.

20. Agricola E, Slavich M, Tufaro V, Fisicaro A, Oppizzi M, Melissano G, et al. Prevalence of thoracic ascending aortic aneurysm in adult patients with known abdominal aortic aneurysm: an echocardiographic study. Int J Cardiol. 2013. pp. 3147-3148.

21. Hultgren R, Larsson E, Wahlgren CM, Swedenborg J. Female and elderly abdominal aortic aneurysm patients more commonly have concurrent thoracic aortic aneurysm. Ann Vasc Surg. 2012;26: 918-923.

22. Rouchaud A, Brandt MD, Rydberg AM, Kadirvel R, Flemming K, Kallmes DF, et al. Prevalence of Intracranial Aneurysms in Patients with Aortic Aneurysms. Am J Neuroradiol. 2016;37: 1664-1668.

23. van 't Hof FN, Ruigrok YM, Lee CH, Ripke S, Anderson G, de Andrade M, et al. Shared Genetic Risk Factors of Intracranial, Abdominal, and Thoracic Aneurysms. J Am Heart Assoc. 2016;14;5. 


\section{Supplemental Material I}

Supplemental Table I. Aneurysm characteristics of identified 36 ECAAs (both complete and incomplete carotid imaging) in the Finnish cohort.

\begin{tabular}{lcccc}
\hline & $\begin{array}{c}\text { Fusiform } \\
\mathbf{n}=\mathbf{2 6}\end{array}$ & $\begin{array}{c}\text { Saccular } \\
\mathbf{n}=\mathbf{1 0}\end{array}$ \\
\hline ECAA related symptoms & $\mathrm{n}(\%)$ & $\mathrm{n}$ & $(\%)$ \\
\hline Ipsilateral ischemic stroke / TIA & 1 & $(4)$ & & $(10)$ \\
\hline Horner's syndrome & 1 & $(4)$ & 1 & $(10)$ \\
\hline Cranial nerve deficit & 2 & $(8)$ & 1 & $(10)$ \\
\hline Asymptomatic & $13 \quad(50)$ & 4 & $(40)$ \\
\hline Unknown & 9 & $(35)$ & 3 & $(30)$ \\
\hline
\end{tabular}

Presumed etiology in radiology report

\begin{tabular}{|c|c|c|c|c|}
\hline Dissection & 6 & $(23)$ & 2 & $(20)$ \\
\hline Connective tissue disease & 1 & (4) & - & \\
\hline Atherosclerosis & 4 & $(15)$ & - & \\
\hline Iatrogenic & 1 & (4) & 1 & $(10)$ \\
\hline Unknown & 14 & $(54)$ & 7 & $(70)$ \\
\hline \multicolumn{5}{|l|}{ Side } \\
\hline Left & 18 & (69) & 5 & $(50)$ \\
\hline Right & 8 & $(31)$ & 5 & $(50)$ \\
\hline Size in $\mathbf{m m}$, median (range) & 9.4 & $(7.4-35.0)$ & 4.3 & $(2.0-7.2)$ \\
\hline \multicolumn{5}{|l|}{ Location IA cerebral circulation } \\
\hline Anterior circulation & 22 & $(85)$ & 8 & $(80)$ \\
\hline Posterior circulation & 3 & (12) & 2 & $(20)$ \\
\hline Both anterior \& posterior circulation & 1 & $(4)$ & - & \\
\hline Multiple IA & 5 & (19) & 1 & $(10)$ \\
\hline Fusiform IA & 1 & (4) & 1 & $(10)$ \\
\hline
\end{tabular}

Data are presented as $n=$ number of aneurysms (\%) unless otherwise indicated. Abbreviations: ECAA = extracranial carotid artery aneurysm, TIA = transient ischemic attack, $\mathrm{mm}=$ millimeter, $\mathrm{IA}$ = intracranial aneurysm. 


\section{Supplemental Material II}

\section{Detailed methods Pourier et al. ${ }^{1}$}

All consecutive patients with an intracranial aneurysm (IA) admitted to or seen the outpatient clinic of the Department of Neurology and Neurosurgery of the University Medical Center Utrecht, the Netherlands, were eligible in the study of Pourier et al. [1]. Patients were included from 1978 up to 2015, with both ruptured and unruptured IAs. Patients were excluded in case of absence of an IA, if the intracranial hemorrhage was perimesencephalic or due to a trauma without a clear IA, or if the IA was associated with an arteriovenous malformation or dural fistula.

Two authors reviewed all available radiological imaging, divided in complete carotid imaging (from aortic arch up to skull base) and partially carotid imaging (one-sided or if only the distal carotid arteries were depicted from the second cervical vertebra i.e. CTA brain). All available carotid imaging was screened for the co-existence of an extracranial carotid artery aneurysm (ECAA). ECAA was defined as a fusiform dilation of the arterial diameter of $\geq 150 \%$ compared with the normal carotid artery diameter, for saccular aneurysms any size was accepted.

\section{Reference}

1. Pourier VEC, van Laarhoven CJHCM, Vergouwen MDI, Rinkel GJE, de Borst GJ. Prevalence of extracranial carotid artery aneurysms in patients with an intracranial aneurysm. PLoS One. 2017;12: e0187479. doi:10.1371/journal.pone.0187479 



\section{Chapter 5}

\section{Systematic review of co-prevalence of}

arterial aneurysms within the vasculature

Eur J Vasc Endovasc Surg 2020; In press.

C.J.H.C.M. van Laarhoven

N.K.N. Jorritsma

J. Balderston

W. Brinjikji

M. Björck

J.A van Herwaarden

G.J. de Borst 


\section{Abstract}

\section{Background and purpose}

Aneurysms are considered focal manifestations of a systemic vascular condition, and various studies report co-prevalence of aneurysms in different vascular beds. Insight in profiles of patients at risk for multiple aneurysms is lacking, and few clinical algorithms exist if additional screening is indicated. This systematic review assessed the co-prevalence of aneurysms in different vascular beds and analyzed putative risk factors for multiple aneurysms.

\section{Methods}

Medline, Embase and Cochrane libraries were searched until February 2020 for studies reporting co-prevalence of aneurysms in different vascular beds by using the keywords: 'aneurysm', 'co-prevalence' or synonyms. All studies were reviewed by two authors independently. Studies were excluded if they described concomitant treatment of multianeurysms, or if the aneurysm was reported solely bilateral, post-dissection, mycotic, traumatic, iatrogenic, or due to a connective tissue disease.

Radar-plots were used to indicate studies that found an association between the investigated features and aneurysm co-prevalence against those that did not find an association.

\section{Results}

Thirty-two studies met the inclusion criteria, describing in total 16,353 patients of whom 2,015 had at least one additional aneurysm. The weighted co-prevalence was 16.9\% [95\% confidence interval (CI) 11.8-22.6], $\mathrm{I}^{2}>90 \%$. At least nineteen combinations of aneurysms were described, mostly derived from retrospective studies. Seventeen of thirty-two (53\%) studies described concurrent aneurysms in patients with an abdominal aortic aneurysm. Predominantly positive associations were found for higher age, hypertension, stenotic disease, presence of $(\geq 3)$ multiple aneurysms, and size of the primary aneurysm.

\section{Conclusion}

Approximately $1 / 6$ patients with a primary aneurysm harbors an additional aneurysm, and $1 / 4$ if the patient has a popliteal artery aneurysm. Higher age, hypertension, stenotic disease, presence of $(\geq 3)$ multiple aneurysms, and size of the primary aneurysm were predictive for aneurysm co-prevalence. These clinical predictors may assist in decisionmaking which patient with a primary aneurysm needs to be screened for additional aneurysms. 


\section{What this paper adds}

Arterial aneurysms are mostly treated as solitary vascular pathologies, though concomitant aneurysmal disease is reported in various arterial beds. This study gives an overview of reported co-prevalence of aneurysms in different vascular territories, and may facilitate the doctor to patient information and consideration for screening of other arterial beds beyond the primary aneurysm. Future consideration of the health-related burden, when the concomitant aneurysm at screening is left untreated, should be performed for clinical implementation of additional screening algorithms.

\section{Introduction}

Aneurysmal dilatations in arterial beds are mostly focal manifestations of a systemic vascular condition, and numerous studies have reported co-prevalence of aneurysms in different arterial beds ${ }^{1-4}$. Although the site of an aneurysm is likely to be subject to anatomical initiating factors, the systemic propensity is determined by environmental and genetic factors ${ }^{1,5}$. A genetic overlap was suggested in aortic aneurysms (AA) and associated with intracranial aneurysms ${ }^{6}$, and peripheral aneurysms ${ }^{7,8}$. In general, aneurysmal risk includes higher age, smoking, sex, but genetic susceptibility, inflammation status and hemodynamics may also contribute to aneurysmal disease ${ }^{1}$. In general, aneurysms are defined as $\geq 150 \%$ dilatation in comparison to the proximal non-affected part of the artery, but diameter thresholds for e.g. abdominal aortic aneurysms (AAA) $\geq 30$ millimeters, have been used and applied widely ${ }^{1,9,10}$.

The updated clinical practice guideline for the management of AAA of the European Society for Vascular Surgery (ESVS) states that screening at 5-10 year interval for AAA may be considered for all men and women with a true peripheral arterial aneurysm (IIb, level C) ${ }^{9}$. The American SVS guideline for AAA also recommends evaluation for an AAA, though only in patients with a popliteal or femoral artery aneurysm ${ }^{10}$. Physical examination that includes the femoral and popliteal arteries is additionally recommended in patients with a suspected or known AAA (1, level A) ${ }^{10}$. The ESVS 2020 guideline for acute limb ischemia is more directive, as screening should be considered in patients treated for a thrombosed popliteal aneurysm with duplex ultrasound imaging every three years of the treated and contralateral arteries, as well as of the aorta, iliac, and femoral arteries (IIa, level C) ${ }^{11}$.

As the different guidelines vary and/or lack insights in risk profile for patients at risk for concurrent aneurysms, and with exception of the latter ${ }^{11}$, type and extensiveness of aneurysm evaluation is left to the readers interpretation. The clinical implementation of these recommendations could therefore vary among treating physicians. In order to give 
an overview of available evidence of co-prevalence of aneurysms in the vasculature, and potentially indicate putative clinical risk factors for concurrent aneurysmal disease, the present systematic review was undertaken.

\section{Methods}

\section{Search strategy and selection criteria}

This systematic review and meta-analysis was performed according to the Preferred Reporting Items for Systematic Reviews and Meta-Analyses (PRISMA) guidelines ${ }^{12}$. Medline, Embase and Cochrane Library databases were systematically searched to identify all studies published until $27^{\text {th }}$ of February 2020 which described a co-prevalence of aneurysms in different vascular beds in patients aged 18-years or older. The keywords 'aneurysm' and 'co-prevalence', and their synonyms were used in the search to select the studies for this review, the full search is listed in Supplemental Table I. The search results were recorded into an electronic database (The EndNote team (2013). EndNote X9, Clarivate Analytics, Philadelphia, PA, United States of America) and duplicates were removed. All articles were screened based on title and abstract by two independent observers (CL, NJ) according to predefined in- and exclusion criteria. Afterwards, the full text of eligible studies was screened to determine the appropriateness for this systematic review (excluded studies after full-text screening are listed in Supplemental Table II). Additional articles were identified by cross-linking. The review was restricted to English-language studies. Disagreements were discussed until consensus was reached. All studies describing original research of co-prevalence of $\geq 2$ aneurysms were eligible for this review. Studies were excluded when reporting on case series $(\leq 20)$ or case reports only; patients aged $\leq 17$-years old; intervention research reporting concomitant treatment of multiple aneurysms; in case of solely bilateral disease (e.g. bilateral popliteal artery aneurysm); or if the aneurysm was mycotic; iatrogenic; post-dissecting; or due to a connective tissue disease.

\section{Data extraction and assessment of methodological quality}

The primary outcome measure was the reported co-prevalence of $\geq 2$ aneurysms, secondary outcome was the availability of putative clinical risk features for co-prevalence. Data that was extracted included: study design, study period, number of patients, type of primary and concurrent aneurysm, co-prevalence ratio, the definition used for aneurysms, type of imaging modality, and availability of univariate and/or multivariate regressed patient-related risk factors for co-prevalence. In order to obtain all adjusted results, both significant and insignificant risk estimates, corresponding authors were contacted for additional data. Included studies were assessed on quality by a modified 
risk of bias tool for prevalence studies ${ }^{13}$ (Table 1). Articles were assessed on Directness of Evidence (DoE) (0-4 points), and Risk of Bias (RoB) (0-8 points). Detailed scoring criteria are listed in Supplemental Table III. The DoE indicates to what extent the study matches the primary research question and the RoB indicates if the study methodologically contains risk of bias. Studies with high DoE (3.5-4 points) and low RoB (6-8 points) are most eligible for the present review. Two authors (CL, NJ) independently assessed methodological quality and carried out data extraction from the individual papers.

\section{Statistical analysis}

The overall co-prevalence was estimated using a random effects model with double arcsine transformation ${ }^{14}$. Weighted means with $95 \%$ confidence interval (CI) of the proportion of patients with aneurysm co-prevalence were calculated by use of MetaXL $5.3^{14}$ (Epigear International Pty Ltd (2011), Sunrise Beach, Queensland, Australia. www. epigear.com). The percentage of variability in estimates attributable to study heterogeneity was quantified by the $\mathrm{I}^{2}$ statistic (\%). The higher the $\mathrm{I}^{2}$ statistic percentage, the more observed study heterogeneity rather than by chance alone ${ }^{15}$. All associated clinical risk features were collected from the separate articles. If a putative feature was reported in fewer than six articles, this feature was excluded from further analysis. A semi-quantitative analysis was performed by plotting studies that found an association between the investigated features and co-prevalence of aneurysms against those that did not find an association. The results with total number of investigated patients were shown in radar graphs. Corresponding authors of included studies that reported adjusted odds ratios (OR) for different patient features were approached and requested to send additional data of all included variables. Obtained data were assessed by meta-analysis using Review Manager (version 5.3.5; Nordic Cochrance Centre, Copenhagen, Denmark). A randomeffects model (Mantel-Haenszel method) was used to account for the heterogeneity in the included observational cohort studies.

\section{Results}

\section{Study selection}

The search identified 7,213 unique articles. After excluding 7,187 articles and adding sixarticles ${ }^{3,16-20}$ by cross-linking, 32 original research articles met the inclusion criteria (Figure 1, Supplemental Table IV). Results of the quality assessment of these 32 studies are presented in Table 1. In total, eight studies ${ }^{19,21-27}(22 \%)$ were assessed as having a high risk of bias in reporting co-prevalence of aneurysms. 
Table 1. Quality assessment table (definitions stated in Supplemental Table III).

\section{Directness of Evidence (DoE)}

Outcome

\begin{tabular}{|c|c|c|c|c|c|c|c|}
\hline & Study (year) & ఏే & 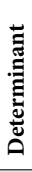 & 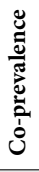 & 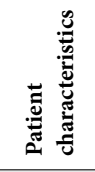 & 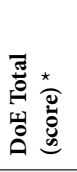 & \\
\hline 1. & Laukka (2019) & $\bullet$ & $\bullet$ & $\bullet$ & $\circ$ & M & (3) \\
\hline 2. & Goyal (2015) & $\bullet$ & $\bullet$ & $\bullet$ & $\odot$ & $\mathrm{H}$ & (3.5) \\
\hline 3. & Malhotra (2019) & $\bullet$ & $\bullet$ & $\bullet$ & $\odot$ & $\mathrm{H}$ & (3.5) \\
\hline 4. & Kuzmik (2010) & $\bullet$ & $\bullet$ & $\bullet$ & $\odot$ & $\mathrm{H}$ & $(3.5)$ \\
\hline 5. & Erben (2019) & $\bullet$ & $\bullet$ & $\bullet$ & $\odot$ & $\mathrm{H}$ & (3.5) \\
\hline 6. & Miyazawa (2007) & $\bullet$ & $\bullet$ & $\bullet$ & $\odot$ & $\mathrm{H}$ & (3.5) \\
\hline 7. & Kurtelius (2019) & $\bullet$ & $\circ$ & $\bullet$ & $\bullet$ & M & (3) \\
\hline 8. & Lee (2017) & $\bullet$ & $\bullet$ & $\bullet$ & $\bullet$ & $\mathrm{H}$ & (4) \\
\hline 9. & Rouchaud (2016) & $\bullet$ & $\bullet$ & $\bullet$ & $\bullet$ & $\mathrm{H}$ & (4) \\
\hline 10. & Shin (2015) & $\bullet$ & $\circ$ & $\bullet$ & $\odot$ & M & $(2.5)$ \\
\hline 11. & Hill (2019) & $\bullet$ & $\bullet$ & $\bullet$ & $\odot$ & $\mathrm{H}$ & $(3.5)$ \\
\hline 12. & van Laarhoven (2020) & $\bullet$ & $\bullet$ & $\bullet$ & $\bullet$ & $\mathrm{H}$ & (4) \\
\hline 13. & Endo (2019) & $\bullet$ & $\bullet$ & $\bullet$ & $\bullet$ & $\mathrm{H}$ & (4) \\
\hline 14. & Stajduhar (1993) & $\bullet$ & $\bullet$ & $\bullet$ & $\circ$ & $\mathrm{H}$ & (3) \\
\hline 15. & Balderston (2015) & $\bullet$ & $\bullet$ & $\bullet$ & $\bullet$ & $\mathrm{H}$ & (4) \\
\hline 16. & DeFreitas (2016) & $\bullet$ & $\bullet$ & $\bullet$ & $\bullet$ & $\mathrm{H}$ & (4) \\
\hline 17. & Dombrowski (2019) & $\bullet$ & $\bullet$ & $\bullet$ & $\odot$ & $\mathrm{H}$ & (3.5) \\
\hline 18. & Wallinder (2018) & $\bullet$ & $\circ$ & $\bullet$ & $\circ$ & $\mathrm{L}$ & (2) \\
\hline 19. & Hultgren (2012) & $\bullet$ & $\bullet$ & $\bullet$ & $\bullet$ & $\mathrm{H}$ & (4) \\
\hline 20. & Chaer (2012) & $\bullet$ & $\bullet$ & $\bullet$ & $\bullet$ & $\mathrm{H}$ & (4) \\
\hline 21. & Chapman (2017) & $\bullet$ & $\bullet$ & $\bullet$ & $\bullet$ & $\mathrm{H}$ & (4) \\
\hline 22. & Hohneck (2019) & $\bullet$ & $\circ$ & $\bullet$ & $\circ$ & $\mathrm{L}$ & (2) \\
\hline 23. & Studziński (2019) & $\bullet$ & $\bullet$ & $\bullet$ & $\odot$ & $\mathrm{H}$ & $(3.5)$ \\
\hline 24. & Armon (1998) & $\bullet$ & $\circ$ & $\odot$ & $\circ$ & $\mathrm{L}$ & (1.5) \\
\hline 25. & Dent (1972) & $\bullet$ & $\bullet$ & $\bullet$ & $\circ$ & $\mathrm{H}$ & (3) \\
\hline 26. & Laine (2017) & $\bullet$ & $\circ$ & $\odot$ & $\circ$ & $\mathrm{L}$ & $(1.5)$ \\
\hline 27. & Graham (1980) & $\bullet$ & $\circ$ & $\bullet$ & $\circ$ & $\mathrm{L}$ & (2) \\
\hline 28. & Cervin (2020) & $\bullet$ & $\bullet$ & $\bullet$ & $\odot$ & $\mathrm{H}$ & $(3.5)$ \\
\hline 29. & Tuveson (2016) & $\bullet$ & $\circ$ & $\bullet$ & $\bullet$ & M & (3) \\
\hline 30. & Diwan (2000) & $\bullet$ & $\bullet$ & $\bullet$ & $\odot$ & $\mathrm{H}$ & $(3.5)$ \\
\hline 31. & Ravn (2017) & $\bullet$ & $\circ$ & $\bullet$ & $\circ$ & $\mathrm{L}$ & (2) \\
\hline 32. & Ravn (2008) & $\bullet$ & • & $\bullet$ & $\bullet$ & $\mathrm{H}$ & (4) \\
\hline & & $\begin{array}{c}\mathrm{L} \\
\mathrm{M} \\
\mathrm{H} \\
\mathrm{n} / \mathrm{r}\end{array}$ & & & $\begin{array}{c}(0-2) \\
(2.5-3) \\
(3.5-4)\end{array}$ & & \\
\hline
\end{tabular}




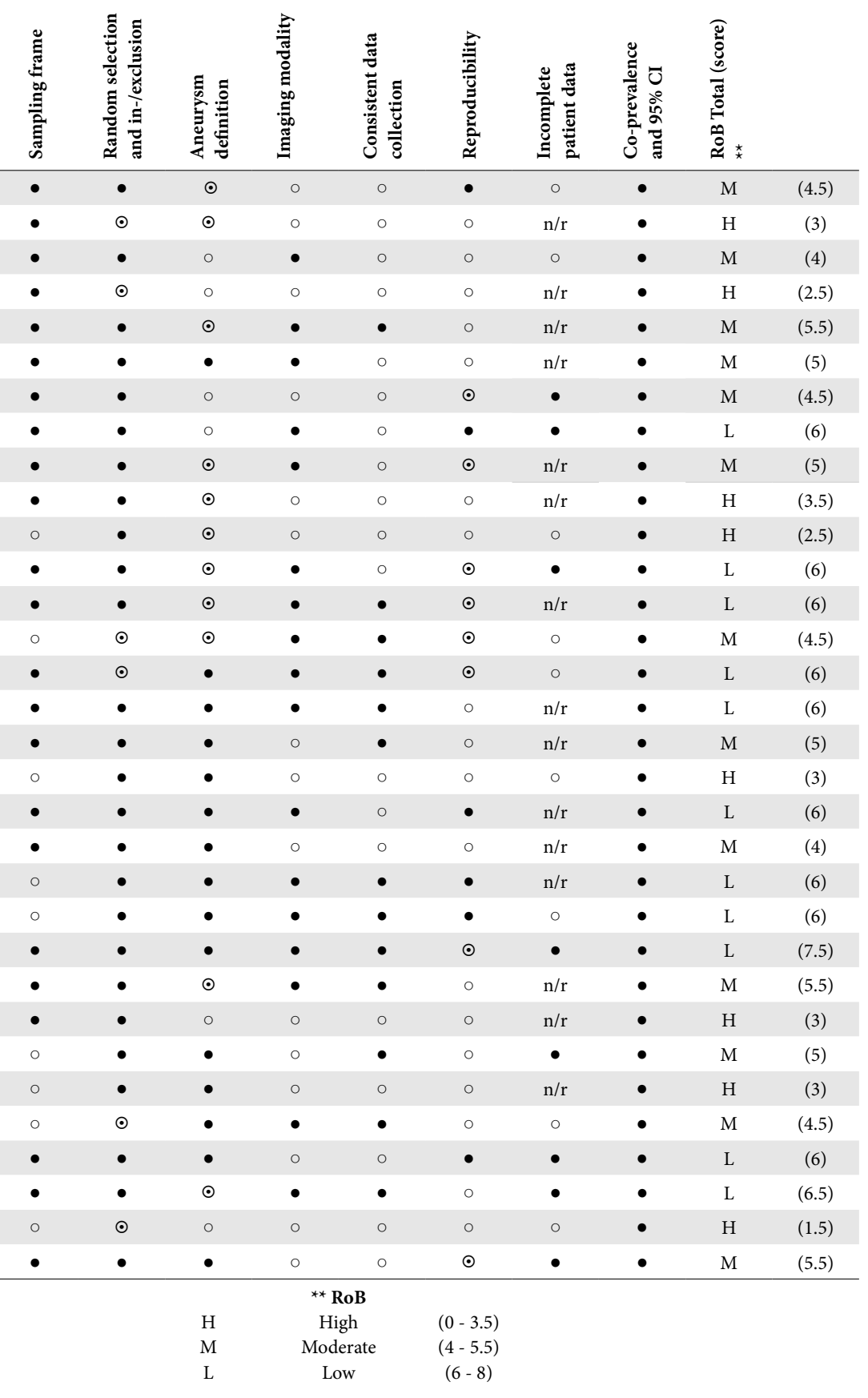




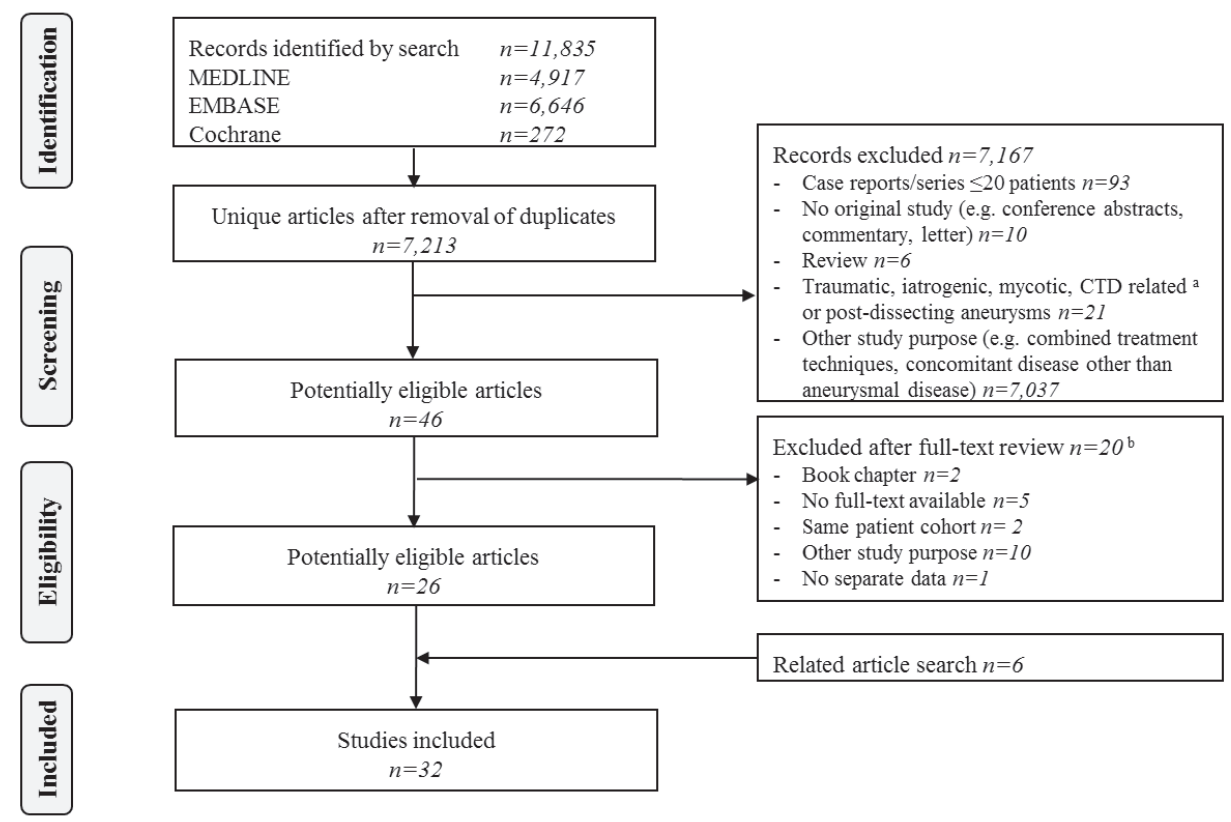

Figure 1. Flowchart of the literature search (February 27, 2020). ${ }^{a}$ Ehlers-Danlos, Loeys-Dietz or Marfan syndrome, fibromuscular dysplasia, or other connective tissue diseases, ${ }^{\mathrm{b}}$ References in Supplemental Table II.

\section{Study sample}

The study characteristics are summarized in Table 2 . In total 16,353 study patients were included, of whom 2,015 had at least one additional aneurysm, representing an overall weighted co-prevalence of $16.9 \%$ (95\% CI 11.8-22.6). Most articles described post-hoc analyses in retrospective cohort studies, and only six studies ${ }^{2,3,28-31}(19 \%)$ were conducted in a prospective manner. Predominately cohorts from Europe (41\%) or United States (47\%) were described. In 21/32 (66\%) articles AA was described as primary diagnosed aneurysm, and in 13/21 (62\%) articles abdominal AA (AAA) in particular. Concurrent aneurysms were found in various studied vascular beds, and more than 19 different combinations of aneurysm co-prevalence were reported. Fifteen studies (47\%) defined both primary and concurrent aneurysms, in contrast to six studies ${ }^{19,22,27,28,32,33}$ (19\%) which did not define any of studied aneurysms. The remaining eleven (34\%) studies defined either only primary ${ }^{23,34}$, or concurrent aneurysm ${ }^{3,16,20,21,25,29,35-37}$ (Supplemental Material II).

The weighted co-prevalence of intracranial aneurysm (IA) and thoracic aortic aneurysm (TAA) was $6.3 \%$ (95\% CI $4.5-8.35)$ with $\mathrm{I}^{2}=55 \%$, for IA and AA (TAA, AAA, or combination of both, within one patient) was $9.3 \%$ (95\% CI 3.0-8.1), $\mathrm{I}^{2}=98 \%$. Coronary artery aneurysms additional to AA disease were investigated in three studies, weighted 


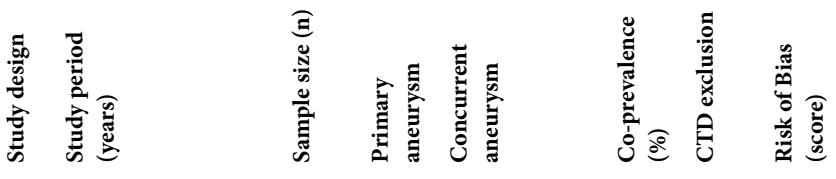

\begin{tabular}{|c|c|c|c|c|c|c|c|c|c|}
\hline 1. Laukka $(2019)^{34}$ & $\mathrm{Re}$ & $2006-2016$ & 411 & sIA & TAA & 7.5 & no & M & $(4.5)$ \\
\hline 2. Goyal $(2015)^{20}$ & $\operatorname{Re}$ & $2002-2011$ & 317 & IA & TAA & 4.7 & no & $\mathrm{H}$ & (3) \\
\hline 3. Malhotra $(2019)^{27}$ & $\mathrm{P}$ & Jun 2009 -May 2018 & 554 & TAA & IA & 4.9 & no & M & $(4)$ \\
\hline \multirow[t]{2}{*}{ 4. $\mathrm{K}$} & $\operatorname{Re}$ & $1997-2009$ & 212 & TAA & IA & 9.0 & no & $\mathrm{H}$ & $(2.5)$ \\
\hline & & & & \multicolumn{2}{|c|}{ Weighted mean } & 6.3 & \multicolumn{3}{|c|}{ (95\% CI 4.5-8.35) } \\
\hline 5. Erben $(2019)^{28}$ & $\mathrm{P}$ & Aug 2011 -Feb 2014 & 81 & IA & AAA & 12.3 & no & M & $(5.5)$ \\
\hline 6. Miyazawa $(2007)^{17}$ & $\mathrm{Re}$ & $\begin{array}{l}\text { Sep } 1997 \text { - } \\
\text { Jul } 2003\end{array}$ & 181 & IA & AAA & 7.2 & no & M & (5) \\
\hline 7. Kurtelius $(2019)^{32}$ & $\operatorname{Re}$ & $1980-2014$ & 4,378 & $\begin{array}{l}\text { sIA } \\
\text { fIA }\end{array}$ & AA & $\begin{array}{l}1.1 \\
14.0\end{array}$ & no & M & $(4.5)$ \\
\hline 8. Lee $(2017)^{31}$ & $\mathrm{Re}$ & $2009-2014$ & 133 & AA & IA & 20.3 & yes & $\mathrm{L}$ & $(6)$ \\
\hline 9. Rouchaud (2016) $)^{33}$ & $\mathrm{Re}$ & Jan 2001 - Jun 2015 & 1,081 & $\mathrm{AA}$ & sIA & 11.8 & yes & M & $(5)$ \\
\hline \multirow[t]{2}{*}{ 10. Shin $(2015)^{22}$} & $\mathrm{Re}$ & $2005-2014$ & 611 & AA & sIA & 11.6 & yes & $\mathrm{H}$ & (3.5) \\
\hline & & & & \multicolumn{2}{|c|}{ Weighted mean } & 9.3 & \multicolumn{3}{|c|}{ (95\% CI 3.0-8.1) } \\
\hline 11. Hill $(2019)^{24}$ & $\mathrm{Re}$ & $2001-2016$ & 31 & RAA & IA & 29 & no & $\mathrm{H}$ & $(2.5)$ \\
\hline 12. van Laarhoven $(2020)^{35}$ & $\operatorname{Re}$ & $2010-2016$ & 1,082 & IA & ECAA & 3.2 & no & $\mathrm{L}$ & $(6)$ \\
\hline 13. Endo $(2019)^{36}$ & $\mathrm{Re}$ & Jan 2014 - Jul 2017 & 123 & AAA & $\mathrm{CAA}$ & 27.6 & no & $\mathrm{L}$ & $(6)$ \\
\hline 14. Stajduhar $(1993)^{19}$ & $\operatorname{Re}$ & Jul 1986 - Oct 1990 & 72 & AAA & CAA & 20.8 & no & M & $(4.5)$ \\
\hline \multirow[t]{2}{*}{ 15. Balderston $(2015)^{37}$} & $\mathrm{Re}$ & Jan 2007 -May 2013 & 477 & AA & CAA & 17 & no & $\mathrm{L}$ & $(6)$ \\
\hline & & & & \multicolumn{2}{|c|}{ Weighted mean } & 22.6 & \multicolumn{3}{|c|}{ (95\% CI 19.0-26.5) } \\
\hline 16. DeFreitas $(2016)^{168}$ & $\mathrm{Re}$ & $2008-2013$ & 462 & TAA & AAA & 22.5 & no & $\mathrm{L}$ & (6) \\
\hline 17. Dombrowski $(2019)^{56}$ & $\operatorname{Re}$ & $2007-2017$ & 218 & AAA & TAA & 18.3 & yes & M & (5) \\
\hline 18. Wallinder $(2018)^{23}$ & $\operatorname{Re}$ & $1982-2013$ & 339 & AAA & TAA and $\mathrm{PAA}^{\$}$ & 31.9 & no & $\mathrm{H}$ & (3) \\
\hline 19. Hultgren $(2012)^{38}$ & $\operatorname{Re}$ & $2004-2008$ & 354 & AAA & TAA & 26.6 & yes & $\mathrm{L}$ & $(6)$ \\
\hline \multirow[t]{2}{*}{ 20. Chaer $(2012)^{39}$} & $\operatorname{Re}$ & $2000-2008$ & 1,082 & AAA & TAA & 23.4 & no & M & $(4)$ \\
\hline & & & & \multicolumn{2}{|c|}{ Weighted mean } & 24.5 & \multicolumn{3}{|c|}{ (95\% CI 20.9-28.3) } \\
\hline 21. Chapman $(2017)^{40 \&}$ & $\operatorname{Re}$ & $2008-2013$ & 371 & TAA & $\mathrm{CIAA}^{\mathrm{s}}$ & 11.1 & no & $\mathrm{L}$ & $(6)$ \\
\hline 22. Hohneck $(2019)^{29}$ & $\mathrm{P}$ & $2018-2019$ & 40 & AAA & CIAA $^{s}$ & 10 & no & $\mathrm{L}$ & $(6)$ \\
\hline 23. Studzińska $(2019)^{57}$ & $\operatorname{Re}$ & Oct 2017 - Jul 2019 & 933 & AAA & $\begin{array}{l}\text { CIAA }^{s} \\
\text { VAA }^{\$}\end{array}$ & 31.7 & no & $\mathrm{L}$ & $(7.5)$ \\
\hline 24. Armon $(1998)^{15}$ & $\operatorname{Re}$ & Jun 1994 - Dec 1996 & 215 & AAA & CIAA & 27.9 & no & M & $(5.5)$ \\
\hline 25. Dent $(1972)^{18 \&}$ & $\mathrm{Re}$ & $1960-1971$ & 1,488 & AAA & $\mathrm{CFAA}^{\$}$, VAA & 3.9 & no & $\mathrm{H}$ & (3) \\
\hline 26. Laine $(2017)^{58}$ & $\operatorname{Re}$ & $2002-2015$ & 60 & IIAA & $\mathrm{AAA}^{\$}$ & 41.7 & no & M & $(5)$ \\
\hline \multirow[t]{2}{*}{ 27. Graham $(1980)^{25 \&}$} & $\mathrm{Re}$ & $1956-1978$ & 100 & CFAA & $\mathrm{AAA}^{\S}$ & 85 & no & $\mathrm{H}$ & (3) \\
\hline & & & & \multicolumn{2}{|c|}{ Weighted mean } & 19.7 & \multicolumn{3}{|c|}{ (95\% CI 3.9-41.9) } \\
\hline
\end{tabular}




\begin{tabular}{|c|c|c|c|c|c|c|c|c|c|}
\hline & 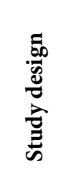 & 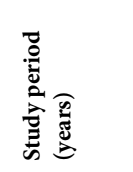 & 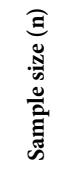 & 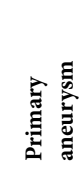 & 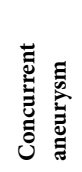 & 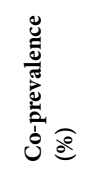 & 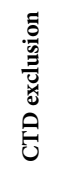 & \multicolumn{2}{|c|}{ 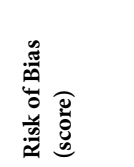 } \\
\hline 28. Cervin $(2020)^{30}$ & $\mathrm{P}$ & $2006-2017$ & 145 & AAA & $\mathrm{PAA}^{\mathrm{s}}$ & 15.9 & no & M & $(4.5)$ \\
\hline 29. Tuveson $(2016)^{59}$ & $\operatorname{Re}$ & $2011-2013$ & 225 & AAA & PAA & 19.1 & no & $\mathrm{L}$ & $(6)$ \\
\hline 30. Diwan $(2000)^{3}$ & $\mathrm{P}$ & $1995-1998$ & 313 & $\mathrm{AAA}$ & PAA & 11.5 & no & $\mathrm{L}$ & $(6.5)$ \\
\hline 31. Ravn $(2017)^{26 \&}$ & $\operatorname{Re}$ & $1987-2012$ & 74 & PAA & $\mathrm{AAA}^{\mathrm{s}}$ & 23.4 & no & $\mathrm{H}$ & $(1.5)$ \\
\hline \multirow[t]{3}{*}{ 32. Ravn $(2008)^{2 \&}$} & $\mathrm{P}$ & $1987-2002$ & 190 & PAA & $\mathrm{AAA}^{\mathrm{s}}$ & 68.9 & no & M & $(5.5)$ \\
\hline & & & & \multicolumn{2}{|c|}{ Weighted mean } & 25.4 & \multicolumn{3}{|c|}{ (95\% CI 4.0-54.6) } \\
\hline & & & Over & I weighte & dmean & 16.9 & \multicolumn{3}{|c|}{ (95\% CI 11.8-22.6) } \\
\hline
\end{tabular}

Abbreviations: Re: retrospective, P: prospective, ${ }^{\&}$ : overlapping inclusion periods, largest study sample included in weighted mean, ${ }^{\$}$ : and other branches. Aneurysms: IA: intracranial aneurysm (sIA: saccular, fIA: fusiform), TAA: thoracic aortic aneurysm, AAA: abdominal aortic aneurysm, AA: aortic aneurysm, RAA: renal artery aneurysm, ECAA: extracranial carotid artery aneurysm, CAA: coronary artery aneurysm, PAA: popliteal artery aneurysm, CIAA: common iliac artery aneurysms, VAA: visceral artery aneurysm, CFAA: common femoral artery aneurysm, IIAA: internal iliac artery aneurysm. Risk of Bias: H: high (0 - 3.5), M: moderate (4 - 5.5), L: low (6 - 8), see also Supplemental Table III.

co-prevalence was $22.6 \%$ (95\% CI 19.0-26.5), $\mathrm{I}^{2}=16 \%$. TAA and AAA were concurrently observed in $24.5 \%$ (95\% CI 20.9-28.3), though $\mathrm{I}^{2}$ was $76 \%$. For common iliac artery and popliteal aneurysms, co-prevalence of $19.7 \%$ (95\% CI 3.9-41.9) and $25.4 \%$ (95\% CI 4.054.6) were found respectively. The heterogeneity between these studies was considerable ${ }^{15}$ $\left(\mathrm{I}^{2}>98 \%\right)$, and due to the small sample sizes wide 95\% CIs were observed. The highest co-prevalence $(85 / 100)$ of $85 \%$ was observed in common femoral artery aneurysm patients with concurrent AAA ( $\mathrm{RoB}=4$, Table 1$)^{26}$. In fourteen studies $(44 \%)$ only one imaging modality was used, while in the remaining eighteen studies multiple imaging modalities and types of scanners were used to confirm the diagnosis of the concurrent aneurysm (Supplemental Material II).

\section{Putative clinical risk factors}

In total, 20/32 studies described clinical risk factors for co-prevalence of aneurysms. An overview of the reported putative clinical risk factors is provided in Table 3 and Table 4, more detailed information on the individual study results can be found in Supplementary Material II. In total, 19 studies described univariate regressed odds ratio's (Table 3), and ten unique patient cohorts reported multivariate adjusted risk estimates for multiple aneurysms (Table 4). Three corresponding authors ${ }^{2,34,38}$ were able to send additional data, and one article ${ }^{39}$ reported both significant and not-significant odds ratios. Pooled data from these four studies ${ }^{2,34,38,39}$ (2,028 patients, $12 \%$ of total study population) is illustrated in Supplemental Figure IA-D. 
Table 3. Results of reported putative risk factors for co-prevalence of $>2$ aneurysms, univariate results.

\begin{tabular}{|c|c|c|c|c|c|c|c|c|c|c|c|c|c|}
\hline Author & $\mathbf{n}$ & 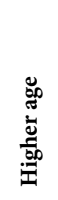 & 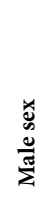 & 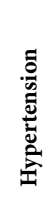 & 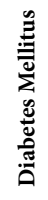 & 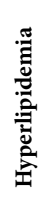 & 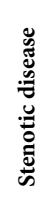 & Оิ & 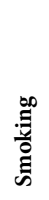 & 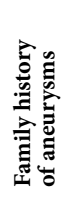 & 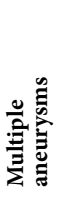 & 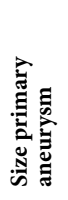 & 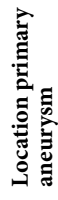 \\
\hline Laukka $(2019)^{34}$ & 411 & & & & & & & & & & & & \\
\hline Goyal $(2015)^{20}$ & 317 & NS & + & + & & & & & NS & & & & \\
\hline Malhotra $(2019)^{27}$ & 554 & NS & NS & NS & & & & & $\mathrm{m}$ & & & & NS \\
\hline Kuzmik $(2010)^{21}$ & 212 & NS & NS & + & & & & & NS & & & & + \\
\hline Erben $(2019)^{28}$ & 81 & NS & NS & NS & NS & NS & NS & & NS & & NS & & \\
\hline Miyazawa $(2007)^{17}$ & 181 & + & + & NS & NS & & NS & & + & NS & + & + & NS \\
\hline Kurtelius $(2019)^{32}$ & 4,378 & & & & & & & & & & & & \\
\hline Lee $(2017)^{31}$ & 133 & + & - & NS & NS & NS & & & NS & & & & + \\
\hline Rouchaud (2016) $)^{33}$ & 1,081 & NS & - & NS & NS & NS & + & & NS & & & + & \\
\hline Shin $(2015)^{22}$ & 611 & NS & + & NS & NS & NS & NS & & NS & & NS & & NS \\
\hline Hill $(2019)^{24}$ & 31 & & & & & & & & & & & & \\
\hline van Laarhoven $(2020)^{35}$ & 1,082 & NS & + & NS & NS & NS & & & NS & NS & & & \\
\hline Endo $(2019)^{36 \%}$ & 123 & & & & & & & & & & & & \\
\hline Stajduhar $(1993)^{19}$ & 72 & & & & & & & & & & & & \\
\hline Balderston $(2015)^{37}$ & $403^{*}$ & + & NS & NS & NS & & NS & & NS & & NS & NS & \\
\hline DeFreitas $(2016)^{16}$ & 462 & + & NS & + & NS & & + & + & + & NS & NS & & \\
\hline Dombrowski $(2019)^{56}$ & 218 & NS & NS & NS & NS & NS & & NS & NS & NS & & NS & \\
\hline Wallinder $(2018)^{23}$ & 339 & & & & & & & & & & & & \\
\hline Hultgren $(2012)^{38}$ & 354 & + & - & + & NS & NS & NS & NS & NS & & & NS & \\
\hline Chaer $(2012)^{39}$ & 1,082 & + & - & + & - & NS & & NS & - & + & & & + \\
\hline Chapman $(2017)^{40}$ & 371 & + & + & + & NS & & + & NS & NS & NS & + & & \\
\hline Hohneck $(2019)^{29}$ & 40 & & & & & & & & & & & & \\
\hline Studzińska $(2019)^{57}$ & 933 & & & & & & & & & & & & \\
\hline Armon $(1998)^{15}$ & 215 & & & & & & & & & & & & \\
\hline Dent $(1972)^{18}$ & 1,488 & & & & & & & & & & & & \\
\hline Laine $(2017)^{58}$ & 60 & & & & & & & & & & & & \\
\hline Graham $(1980)^{25}$ & 100 & & & & & & & & & & & & \\
\hline Cervin $(2020)^{30}$ & $145^{*}$ & & & & & & & & & & & NS & \\
\hline Tuveson $(2016)^{59}$ & 225 & NS & NS & & NS & & + & NS & NS & & & & \\
\hline Diwan $(2000)^{3}$ & 313 & NS & + & NS & NS & NS & + & & NS & NS & & & \\
\hline $\operatorname{Ravn}(2017)^{26}$ & 74 & & & & & & & & & & & & \\
\hline $\operatorname{Ravn}(2008)^{2}$ & 190 & + & NS & + & & & & & NS & NS & $+^{*}$ & & \\
\hline
\end{tabular}

Abbreviations: $\mathrm{n}$ : number of study patients, ${ }^{*}$ : subgroup analysis, ${ }^{\%}$ : analyzed propensity score matched cohort without aneurysm patients, + : positive, -: negative association with co-prevalence of aneurysms, NS: not-significant, $\mathrm{m}$ : missing data in $>30 \%$ of patients. 
Table 4. Results of reported putative risk factors for co-prevalence of $>2$ aneurysms, multivariate regressed results.

\begin{tabular}{|c|c|c|c|c|c|c|c|c|c|c|c|c|c|}
\hline Author & $\mathbf{n}$ & 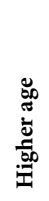 & 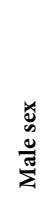 & 这通 & 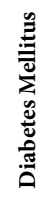 & & 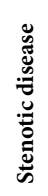 & อิ & 曷 & 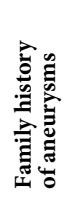 & 莺 & 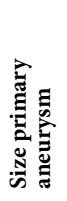 & 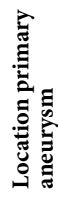 \\
\hline Laukka $(2019)^{34}$ & 411 & & & & & & & & & & & & \\
\hline Goyal $(2015)^{20}$ & 317 & & & & & & & & & & & & \\
\hline Malhotra $(2019)^{27}$ & 554 & & & & & & & & & & & & \\
\hline $\operatorname{Kuzmik}(2010)^{21}$ & 212 & & & & & & & & & & & & \\
\hline Erben $(2019)^{28}$ & 81 & & & & & & & & & & & & \\
\hline Miyazawa $(2007)^{17 \#}$ & 181 & & & & & & & & & & & & \\
\hline Kurtelius $(2019)^{32}$ & 4,378 & & + & NS & NS & + & + & & & & & & \\
\hline Lee $(2017)^{31}$ & 133 & NS & NS & & & & & & & & & & NS \\
\hline Rouchaud (2016) & 1,081 & & - & & & & NS & & & & & NS & NS \\
\hline Shin $(2015)^{22}$ & 611 & & & & & & & & & & & & \\
\hline Hill $(2019)^{24}$ & 31 & & & & & & & & & & & & \\
\hline van Laarhoven $(2020)^{35}$ & $1,048^{*}$ & NS & + & NS & NS & NS & & & & & & & \\
\hline Endo $(2019)^{36 \%}$ & 123 & & & & & & & & & & & & \\
\hline Stajduhar $(1993)^{19}$ & 72 & & & & & & & & & & & & \\
\hline Balderston $(2015)^{37}$ & $403^{*}$ & NS & NS & NS & NS & & NS & & NS & & NS & NS & \\
\hline DeFreitas $(2016)^{16}$ & 462 & + & + & + & & & & & + & & & & + \\
\hline Dombrowski $(2019)^{56}$ & 218 & & & & & & & & & & & & \\
\hline Wallinder $(2018)^{23}$ & 339 & & & & & & & & & & & & \\
\hline Hultgren $(2012)^{38}$ & 354 & + & - & NS & NS & & NS & NS & NS & & & & \\
\hline Chaer $(2012)^{39}$ & 1,082 & & NS & + & - & NS & NS & NS & - & + & & + & - \\
\hline Chapman $(2017)^{40}$ & 371 & + & + & & & & & & & & + & & \\
\hline Hohneck $(2019)^{29}$ & 40 & & & & & & & & & & & & \\
\hline Studzińska $(2019)^{57}$ & 933 & & & & & & & & & & & & \\
\hline Armon $(1998)^{15}$ & 215 & & & & & & & & & & & & \\
\hline Dent $(1972)^{18}$ & 1,488 & & & & & & & & & & & & \\
\hline Laine $(2017)^{58}$ & 60 & & & & & & & & & & & & \\
\hline Graham $(1980)^{25}$ & 100 & & & & & & & & & & & & \\
\hline Cervin $(2020)^{30}$ & $145^{*}$ & & & & & & & & & & & & \\
\hline Tuveson $(2016)^{59}$ & 225 & & & & & & & & & & & & \\
\hline Diwan $(2000)^{3}$ & 313 & & & & & & & & & & & & \\
\hline $\operatorname{Ravn}(2017)^{26}$ & 74 & & & & & & & & & & & & \\
\hline $\operatorname{Ravn}(2008)^{2}$ & 190 & + & NS & + & & & & & NS & NS & & & \\
\hline
\end{tabular}

Abbreviations: $\mathrm{n}$ : number of study patients, ${ }^{*}$ : subgroup analysis, ${ }^{*}$ : adjusted for 12 covariates in $<15$ number of events, ${ }^{\%}$ : analyzed propensity score matched cohort without aneurysm patients, +: positive, -: negative association with co-prevalence of aneurysms, NS: not-significant. 
The relationship of studies reporting significant and not-significant risk estimates is illustrated in Figure 2. For higher age, hypertension, stenotic disease, presence of $(\geq 3)$ multiple aneurysms, and size of the primary aneurysm only positive or no associations were found throughout all studies. Only one positive association was found for chronic obstructive pulmonary disease (COPD) ${ }^{17}$, but contradicted by adjusted results ${ }^{39,40}$. Family history of aneurysms was only positively associated once ${ }^{40}$, and further no associations were found. For male sex, smoking, and location of primary aneurysm were positive, negative, and not-significant associations found. Male sex was only negatively associated with patients harboring an AA of AAA, and co-prevalence of an $\mathrm{IA}^{32,34}$ or TAA ${ }^{39,40}$ respectively. Both positive and negative risk estimates for higher age, male sex, hypertension and smoking were also observed when pooling crude estimates from obtained additional data (Supplemental Figure I A-D). For diabetes mellitus only one negative ${ }^{40}$ and further no associations were reported, and for hyperlipidemia only one large study ${ }^{33}$ found a positive association while using a competing risks multivariate Cox regression model consisting of 4,378 patients (Table 4, Figure 2B). For higher age, four out of seven studies showed a positive association with aneurysm co-prevalence after multivariate regression (Table 4). However, these studies represented a small number of patients $\left(1,377^{2,17,39,41}\right.$ vs $\left.1,584^{32,36,38}\right)$ (Fig 2B). Location of the primary aneurysm showed in two studies a significant association, though in 1,082 patients ${ }^{40}$ negative, and in only $462^{17}$ patients positive odds. After adjustment, only COPD showed no association throughout ( 2 out of 2 studies ${ }^{39,40}$ ).

\section{Discussion}

This systematic review (32 studies, 16,353 patients) showed that approximately one out of six patients with a primary aneurysm harbors a concurrent aneurysm, and one in four if the patient has a popliteal aneurysm. Various cardiovascular risk features were analyzed in the included studies, though none of the individual characteristics showed an unambiguous risk effect. Only positive or no associations were found for higher age, hypertension, stenotic disease, presence of $(\geq 3)$ multiple aneurysms, and size of the primary aneurysm. Female sex was associated with co-prevalence of AA or AAA patients in 2/10 studies, where patients additionally harbored an IA or TAA, respectively. In total, more than 19 combinations of different aneurysms were described, but mostly derived from post-hoc analyses in retrospective cohort studies.

Additional aneurysm screening is recommended in different guidelines ${ }^{9-11}$, though the extensiveness and time intervals of screening vary. The clinical implementation may therefore differ per treating physician. The evidence supporting these recommendations 
A

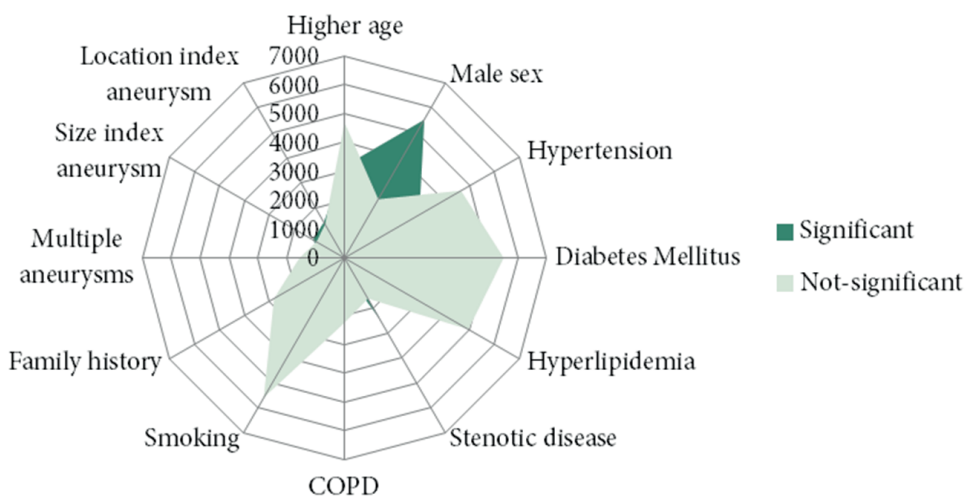

B

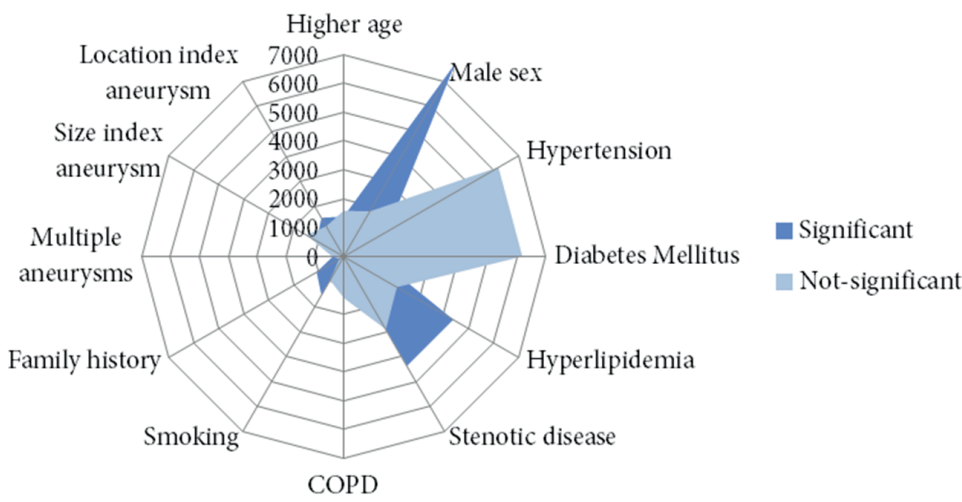

Figure 2. Radar graphs of the included univariate (A, 19 studies) and multivariate (B, 10 studies) regressed putative clinical risk features analyzed in studies reporting co-prevalence of aneurysms. Studies that found a significant association (dark colored) were plotted against studies finding a not-significant association (light colored). The y-axis represents the cumulative number of study patients; each axis of the circular radar graph represents one clinical risk factor.

is included in this review ${ }^{2,3,26}$. Age $\geq 65$-years-old, male sex, smoking, hypertension, atherosclerosis, ethnicity, and family history of AAA are well established clinical risk factors associated with presence of $\mathrm{AAA}^{42-46}$. Diabetes, in particular diabetes mellitus type 2, seems to prevent development of $\mathrm{AAA}^{47}$. Despite comprehensive discussion of risk factors for AAA development and outcome after treatment, no separate insight in risk profile for concurrent aneurysms in the guidelines is given. This review shows that pooling reliable risk estimates from the available co-prevalence studies is not straightforward. Applied imaging modalities, definitions of included aneurysms and/or clinical risk features vary, resulting in heterogeneous study results (Supplemental Material II). To account for inherent differences, no direct comparisons in all included studies were made. However, based on this review treating physicians should consider additional 
aneurysm screening in typical vascular patients (elderly male with hypertension and stenotic disease), and in case of presence of ( $\geq 3)$ multiple aneurysms and large size of the primary aneurysm. In addition, female AA patients seem more susceptible for TAA or IA, and if they have IA they also have an increases risk of popliteal artery aneurysm ${ }^{23}$. Perhaps additional screening should be based on related health-burden when the concurrent aneurysm at screening is left untreated. The screening of AAA in patients with an IA was reported to be cost-effective in a American hypothetical cohort $^{48}$. In addition to important financial aspects, quality of life in patients $\mathrm{s}^{49}$ harboring multiple aneurysms, including aneurysms that do not require treatment, should be taken into account in additional screening guidelines. Although aneurysm rupture rate and hospital costs are subject to geographic study location, similar simulation studies could be rewarding in selecting which artery should be screened additionally. Fairly benign concomitant aneurysms at screening (e.g. coronary or extracranial carotid artery aneurysms) with low-risk for adverse outcome will be excluded, and sophisticated screening recommendations for additional high-risk aneurysms will subsequently be generated.

Of the six prospective studies, only one reported adjusted risk estimates ${ }^{2}$, probably partly due to small number of events and thus statistical power, ${ }^{3,28-31}$. Higher age and hypertension at baseline were independently associated with extra aneurysms in the Swedish analysis of 190 patients with popliteal artery aneurysms who were re-examined after a median of seven years ${ }^{2}$. Somewhat similar results were observed in our meta-analyses, although $\mathrm{I}^{2}$ statistic was considerable for age and male sex (Supplemental Figure 1A,B). Presence of $\geq 3$ aneurysms was observed in various studies. It seems logical that harboring multiple aneurysms is associated to co-prevalence of even more arterial aneurysms, though the question remains when having either one aneurysm or more, whether all dilatations are caused by the same aneurysm-prone vascular subtype. As connective tissue diseases like Marfan and Ehlers-Danlos syndromes are rarely tested and diagnosed ${ }^{50}$, it is reasonable to assume that these and other yet to be discovered genetic vascular types contribute to aneurysm development. A shared genetic base was shown in twin studies ${ }^{51,52}$ and first grade relatives with $\mathrm{AAA}^{53,54}$ and $\mathrm{IA}^{55}$, indicating that family history affects future aneurysm development. Future research should include first relative family history for other arterial aneurysm types. Some evidence has been raised that patients with an AAA have peripheral arteriomegaly ${ }^{56}$, this may be an explanation why size of the primary aneurysm is associated to co-prevalence of concurrent aneurysms, in particular when absolute values are used for aneurysm definition. Large aneurysm size also indicates advanced aneurysmal disease and contributing risk factors could have dilated other vascular territories simultaneously. 


\section{Limitations and future perspectives}

Due to the broad nature of our research question, it is inevitable that other informative articles were missed. Extensive cross-linking was performed to minimize this. Multiple aneurysms within the same artery were left out of this analysis in attempt to analyze aneurysmal disease in different vascular beds, though defining a cut-off point between arteries is arbitrarily and subject to the study properties. The influence of connective tissue diseases (CTD) on aneurysmal disease was minimized by excluding articles describing solely CTD-related aneurysms, though it is imaginable that remaining CTDpatients are included in the present review, as genetic testing to confirm the diagnosis is subject to local practice. Further, the majority of included papers performed a post-hoc analysis in a retrospective database. Many of the study patients were selected from a surgically driven cohort, and associated risk features could have been taken care of (e.g. smoking cessation), and as a result their influence may be less pronounced. The timing of enrollment (first diagnosis or after surgery), and more importantly timing and indication for additional imaging of specific region of interest, is not addressed in many studies and may contribute to selection bias. Moreover, in retrospective studies the time of diagnosis of both aneurysms could differ largely, and the cross-sectional design for prevalence estimation may be violated. The difference in prevalence or incidence should therefore be emphasized, as the risk features associated to them may also be timedependent e.g. recent infection, surgery, arterial dissection. Only one prospective study included time-to-diagnosis in their analyses ${ }^{2}$. Even though retrospective co-prevalence studies are a reflection of daily clinical practice, associated etiological results are hampered by this. Additionally, we were only able to pool crude data from four studies despite extensive contact with corresponding authors. Due to the observed heterogeneity in the co-prevalence studies, pooling data by an individual patient data meta-analysis was not feasible. Future studies aiming to unravel concurrent aneurysmal disease, should ideally be performed prospectively, include the time-to-diagnosis in their analyses, and screen the region of interests in all study participants with a similar modality scored independently by two trained observers, in attempt to rule out residual bias. As indicated previously, heritability of aneurysms is estimated high, but the largest genome-wide association study to date only includes 4,972 AAA patients ${ }^{5}$. Indication of other aneurysm-prone genetic profiles may improve indication of high-risk patients, in addition to established clinical features. In this manner, detailed patient-related risk features for specific subtypes of arterial aneurysm co-prevalence can be identified. 


\section{Conclusions}

Approximately one out of six patients with a primary aortic aneurysm harbors a concurrent aneurysm, and one in four if the patient has a popliteal artery aneurysm. Higher age, hypertension, stenotic disease, presence of $(\geq 3)$ multiple aneurysms, and size of the primary aneurysm seem predictive for aneurysm co-prevalence. These clinical predictors may assist in decision-making which patient with a primary aneurysm needs to be screened for additional aneurysms.

\section{Acknowledgements}

The authors greatly acknowledge the contributions of all authors of the included studies, a special thanks to Dr. Zachary Gertz. 


\section{References}

1. Norman PE, Powell JT. Site specificity of aneurysmal disease. Circulation 2010;121:560-8.

2. Ravn H, Wanhainen A, Björck M. Risk of new aneurysms after surgery for popliteal artery aneurysm. $\mathrm{Br} J$ Surg 2008;95:571-5.

3. Diwan A, Sarkar R, Stanley JC, et al. Incidence of femoral and popliteal artery aneurysms in patients with abdominal aortic aneurysms. J Vasc Surg 2000;31:863-9.

4. Dawson I, Sie RB, Van Bockel JH. Atherosclerotic popliteal aneurysm. Br J Surg 1997;84:293-9.

5. Jones GT, Tromp G, Kuivaniemi H, et al. Meta-Analysis of Genome-Wide Association Studies for Abdominal Aortic Aneurysm Identifies Four New Disease-Specific Risk Loci. Circ Res 2017;120:341-53.

6. van't Hof FNG, Ruigrok YM, Lee $\mathrm{CH}$, et al. Shared genetic risk factors of intracranial, abdominal, and thoracic aneurysms. J Am Heart Assoc 2016;5:1-19.

7. Fiotti N, Calvagna C, Sgorlon G, et al. Multiple sites of vascular dilation or aneurysmal disease and matrix metalloproteinase genetic variants in patients with abdominal aortic aneurysm. J Vasc Surg 2018;67:1727-35.

8. van Laarhoven CJHCM, van Setten J, van Herwaarden JA, et al. Polygenic Susceptibility of Aortic Aneurysms Associates to the Diameter of the Aneurysm Sac: the Aneurysm-Express Biobank Cohort. Sci Rep 2019;27;9:19844.

9. Wanhainen A, Verzini F, Van Herzeele I, et al. Editor's Choice - European Society for Vascular Surgery (ESVS) 2019 Clinical Practice Guidelines on the Management of Abdominal Aorto-iliac Artery Aneurysms. Eur J Vasc Endovasc Surg 2019;57:8-93.

10. Chaikof EL, Dalman RL, Eskandari MK, et al. The Society for Vascular Surgery practice guidelines on the care of patients with an abdominal aortic aneurysm. J Vasc Surg 2018;67:2-77.e2.

11. Björck M, Earnshaw JJ, Acosta S, et al. Editor's Choice - European Society for Vascular Surgery (ESVS) 2020 Clinical Practice Guidelines on the Management of Acute Limb Ischaemia. Eur J Vasc Endovasc Surg 2020;59:173-218.

12. Moher D, Liberati A, Tetzlaff J, et al. Preferred reporting items for systematic reviews and meta-analyses: The PRISMA statement. PLoS Med 2009;6.

13. Hoy D, Brooks $\mathrm{P}$, Woolf $\mathrm{A}$, et al. Assessing risk of bias in prevalence studies: Modification of an existing tool and evidence of interrater agreement. J Clin Epidemiol 2012;65:934-9.

14. Barendregt JJ, Doi SA, Lee YY, et al. Meta-analysis of prevalence. J Epidemiol Community Health 2013;67:974-8.

15. Higgins JPT, Thomas J, Chandler J, Cumpston M, Li T, Page MJ, Welch V. Cochrane Handbook for Systematic Review of Interventions version 6.0. Cochrane 2019.

16. Armon MP, Wenham PW, Whitaker SC, et al. Common iliac artery aneurysms in patients with abdominal aortic aneurysms. Eur J Vasc Endovasc Surg 1998;15:255-7.

17. DeFreitas MR, Quint LE, Watcharotone K, et al. Evaluation for abdominal aortic aneurysms is justified in patients with thoracic aortic aneurysms. Int J Cardiovasc Imaging 2016;32:647-53.

18. Miyazawa N, Akiyama I, Yamagata Z. Risk factors for the association of intracranial and aortic aneurysms. Acta Neurochir(Wien) 2007;149:221-9.

19. Dent TL, Lindenauer SM, Ernst CB, et al. Multiple Arteriosclerotic Arterial Aneurysms. Arch Surg 1972;105:338-44.

20. Stajduhar MAJKC, Laird MAJJR, Rogan KM, et al. Coronary artery ectasia: Increased prevalence in patients with abdominal aortic aneurysm as compared to occlusive atherosclerotic peripheral vascular disease. Am Heart J 1993;125:86-92.

21. Goyal MS, Gottumukkala R, Bhalla S, et al. Bicuspid aortic valves and thoracic aortic aneurysms in patients with intracranial aneurysms. Neurology 2015;84:46-9.

22. Kuzmik GA, Feldman M, Tranquilli M, et al. Concurrent Intracranial and Thoracic Aortic Aneurysms. Am J Cardiol 2010;105:417-20.

23. Shin YW, Jung KH, Moon J, et al. Site-Specific Relationship between Intracranial Aneurysm and Aortic Aneurysm. Stroke 2015;46:1993-6.

24. Wallinder J, Georgiou A, Wanhainen A, et al. Prevalence of Synchronous and Metachronous Aneurysms in Women With Abdominal Aortic Aneurysm. Eur J Vasc Endovasc Surg 2018;56:435-40. 
25. Hill HL, Stanley JC, Matusko N, et al. The Association of Intracranial Aneurysms in Women with Renal Artery Aneurysms. Ann Vasc Surg 2019;60:147-155.e2.

26. Graham LM, Zelenock GB, Whitehouse WM, et al. Clinical Significance of Arteriosclerotic Femoral Artery Aneurysms. Arch Surg 1980;115:502-7.

27. Ravn H, Pansell-Fawcett K, Björck M. Popliteal Artery Aneurysm in Women. Eur J Vasc Endovasc Surg 2017;54:738-43.

28. Malhotra A, Seifert K, Wu X, et al. Screening for Intracranial Aneurysms in Patients with Thoracic Aortic Aneurysms. Cerebrovasc Dis 2019;47:253-9.

29. Erben Y, Barrett KM, Freeman WD, et al. Prevalence of Previously Undiagnosed Abdominal Aortic Aneurysms in Patients with Intracranial Aneurysms: From the Brain and Aortic Aneurysms Study (BAAS). Neurocrit Care 2020;32: 796-803.

30. Hohneck A, Keese M, Ruemenapf G, et al. Prevalence of abdominal aortic aneurysm and associated lower extremity artery aneurysm in men hospitalized for suspected or known cardiopulmonary disease. BMC Cardiovasc Disord 2019;19:1-7.

31. Cervin A, Wanhainen A, Björck M. Popliteal Aneurysms are Common Among Men With Screening Detected Abdominal Aortic Aneurysms, and Prevalence Correlates With the Diameters of the Common Iliac Arteries. Eur J Vasc Endovasc Surg 2020;59:67-72.

32. Lee D, Ahn SJ, Cho ES, et al. High prevalence of intracranial aneurysms in patients with aortic dissection or aneurysm: Feasibility of extended aorta CT angiography with involvement of intracranial arteries. $J$ Neurointerv Surg 2017;9:1017-21.

33. Kurtelius A, Väntti N, Rezai Jahromi B, et al. Association of Intracranial Aneurysms With Aortic Aneurysms in 125 Patients With Fusiform and 4253 Patients With Saccular Intracranial Aneurysms and Their Family Members and Population Controls. J Am Heart Assoc 2019;8:e013277.

34. Rouchaud A, Brandt MD, Rydberg AM, et al. Prevalence of intracranial aneurysms in patients with aortic aneurysms. Am J Neuroradiol 2016;37:1664-8.

35. Laukka D, Pan E, Fordell T, et al. Prevalence of thoracic aortic aneurysms and dilatations in patients with intracranial aneurysms. J Vasc Surg 2019;70:1801-8.

36. van Laarhoven CJHCM, Pourier VEC, Lindgren AE, et al. Co-prevalence of extracranial carotid aneurysms differs between European intracranial aneurysm cohorts. PLoS One 2020;15:e0228041.

37. Endo H, Dohi T, Dohi S, et al. Clinical indicators and coronary angiographic features of expansive arterial remodelling in patients with abdominal aortic aneurysms. PLoS One 2019;14:e0219730.

38. Balderston JR, Giri J, Kolansky DM, et al. Coronary artery aneurysms associated with ascending aortic aneurysms and abdominal aortic aneurysms: Pathophysiologic implications. Catheter Cardiovasc Interv 2015;85:961-7.

39. Hultgren R, Larsson E, Wahlgren CM, et al. Female and elderly abdominal aortic aneurysm patients more commonly have concurrent thoracic aortic aneurysm. Ann Vasc Surg 2012;26:918-23.

40. Chaer RA, Vasoncelos R, Marone LK, et al. Synchronous and metachronous thoracic aneurysms in patients with abdominal aortic aneurysms. J Vasc Surg 2012;56:1261-5.

41. Chapman M, Quint LE, Watcharotone K, et al. Pelvic artery aneurysm screening provides value in patients with thoracic aortic aneurysms. Int J Cardiovasc Imaging 2017;33:1627-35.

42. Jahangir E, Lipworth L, Edwards TL, et al. Smoking, sex, risk factors and abdominal aortic aneurysms: a prospective study of 18782 persons aged above 65 years in the Southern Community Cohort Study. Physiol Behav 2017;176:139-48.

43. Kent KC, Zwolak RM, Egorova NN, et al. Analysis of risk factors for abdominal aortic aneurysm in a cohort of more than 3 million individuals. J Vasc Surg 2010;52:539-48.

44. Stackelberg O, Björck M, Larsson SC, et al. Sex differences in the association between smoking and abdominal aortic aneurysm. Br J Surg 2014;101:1230-7.

45. Svensjö S, Björck M, Gürtelschmid M, et al. Low prevalence of abdominal aortic aneurysm among 65-yearold swedish men indicates a change in the epidemiology of the disease. Circulation 2011;124:1118-23.

46. Singh K, Bønaa KH, Jacobsen BK, et al. Prevalence of and risk factors for abdominal aortic aneurysms in a population-based study: The Tromsø study. Am J Epidemiol 2001;154:236-44.

47. Shah AD, Langenberg C, Rapsomaniki E, et al. Type 2 diabetes and incidence of cardiovascular diseases: A cohort study in 1.9 million people. Lancet Diabetes Endocrinol 2015;3:105-13. 
48. Ball BZ, Jiang B, Mehndiratta P, et al. Screening individuals with intracranial aneurysms for abdominal aortic aneurysms is cost-effective based on estimated coprevalence. J Vasc Surg 2016;64:811-818.e3.

49. Lyttkens L, Wanhainen A, Svensjö S, et al. Systematic Review and Meta-Analysis of Health Related Quality of Life and Reported Experiences in Patients With Abdominal Aortic Aneurysm Under Ultrasound Surveillance. Eur J Vasc Endovasc Surg 2020;59:420-7.

50. Aday AW, Kreykes SE, Fanola CL. Vascular Genetics: Presentations, Testing, and Prognostics. 2018;20:103.

51. Joergensen TMM, Christensen K, Lindholt JS, et al. Editor's Choice - High Heritability of Liability to Abdominal Aortic Aneurysms: A Population Based Twin Study. Eur J Vasc Endovasc Surg 2016;52:41-6.

52. Wahlgren CM, Larsson E, Magnusson PKE, et al. Genetic and environmental contributions to abdominal aortic aneurysm development in a twin population. J Vasc Surg 2010;51:3-7.

53. Van De Luijtgaarden KM, Rouwet E V., Hoeks SE, et al. Risk of abdominal aortic aneurysm (AAA) among male and female relatives of AAA patients. Vasc Med (United Kingdom) 2017;22:112-8.

54. van de Luijtgaarden KM, Heijsman D, Maugeri A, et al. First genetic analysis of aneurysm genes in familial and sporadic abdominal aortic aneurysm. Hum Genet 2015;134:881-93.

55. Vlak MHM, Algra A, Brandenburg R, et al. Prevalence of unruptured intracranial aneurysms, with emphasis on sex, age, comorbidity, country, and time period: A systematic review and meta-analysis. Lancet Neurol 2011;10:626-36.

56. Johnsen SH, Joakimsen O, Singh K, et al. Relation of common carotid artery lumen diameter to general arterial dilating diathesis and abdominal aortic aneurysms: The Tromsø study. Am J Epidemiol 2009;169:330-8.

57. Dombrowski D, Long GW, Chan J, et al. Screening Chest Computed Tomography is Indicated in All Patients with Abdominal Aortic Aneurysm. Ann Vasc Surg 2020;65:190-5.

58. Studzińska D, Rudel B, Polok K, et al. Infrarenal versus Suprarenal Abdominal Aortic Aneurysms: Comparison of Associated Aneurysms and Renal Artery Stenosis. Ann Vasc Surg 2019;58:248-254.

59. Laine MT, Björck M, Beiles CB, et al. Few internal iliac artery aneurysms rupture under $4 \mathrm{~cm}$. J Vasc Surg 2017;65:76-81.

60. Tuveson V, Löfdahl HE, Hultgren R. Patients with abdominal aortic aneurysm have a high prevalence of popliteal artery aneurysms. Vasc Med (United Kingdom) 2016;21:369-75. 


\title{
Supplemental Material I
}

\author{
A: Higher age
}

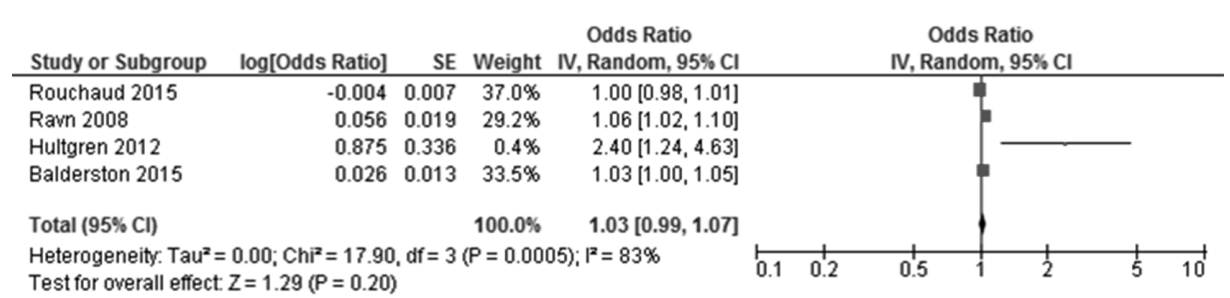

B: Male sex

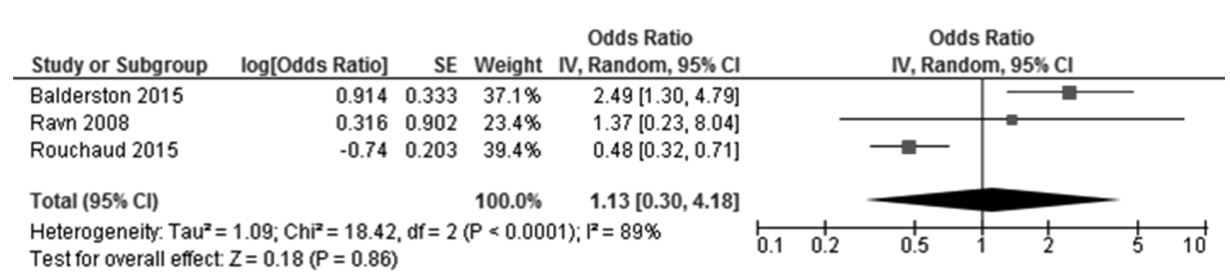

\section{C: Hypertension}

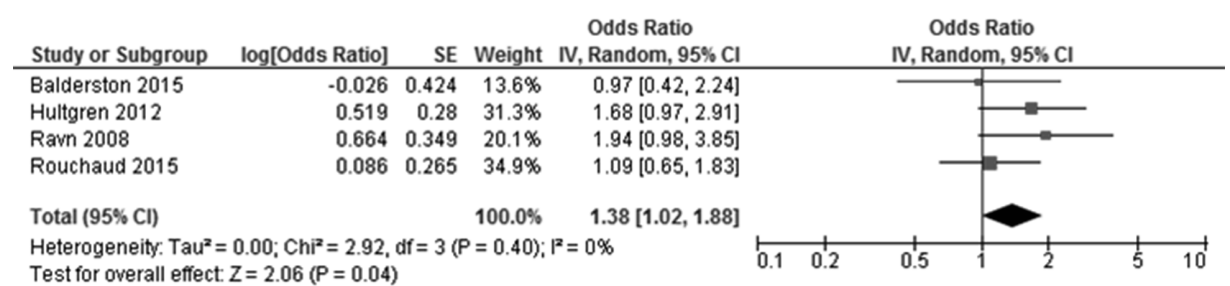

D: Smoking

Odds Ratio

Odds Ratio

Study or Subgroup log[Odds Ratio] SE Weight IV, Random, 95\% Cl

\begin{tabular}{|c|c|c|c|c|}
\hline Balderston 2015 & 0.022 & 0.294 & $22.6 \%$ & $1.02[0.57,1.82]$ \\
\hline Hultgren 2012 & -0.128 & 0.297 & $22.1 \%$ & $0.88[0.49,1.57]$ \\
\hline Ravn 2008 & 0.502 & 0.412 & $11.5 \%$ & $1.65[0.74,3.7$ \\
\hline Rouchaud 2015 & 0.298 & 0.211 & $43.8 \%$ & $1.35[0.89,2$. \\
\hline Total $(95 \% \mathrm{Cl})$ & & & $100.0 \%$ & $1.18[0.90,1$. \\
\hline
\end{tabular}

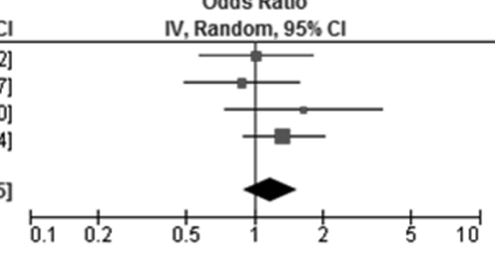

Supplemental Figure I. Meta-analysis of clinical risk features for co-prevalence of arterial aneurysms, additional data from four included studies. 
Supplemental Table I. Full electronic search strategy (conducted at $27^{\text {th }}$ of February, 2020).

\begin{tabular}{|c|c|c|}
\hline Database & Search & Results \\
\hline Medline & $\begin{array}{l}((((((((\text { concomitant}[\text { Title/Abstract] }) \text { OR prevalence[Title/Abstract] }) \text { OR co } \\
\text { prevalence[Title/Abstract] }) \text { OR coexistent[Title/Abstract] }) \text { OR concordant[Title/Abstract] }) \\
\text { OR conjoined[Title/Abstract] }) \text { OR multianeurysm[Title/Abstract] }) \text { OR synchron*[Title/ } \\
\left.\left.\text { Abstract] }) \text { OR accessor* }{ }^{*} \text { Title/Abstract] }\right) \text { AND aneurysm*[Title/Abstract] }\right)\end{array}$ & 4,917 \\
\hline Embase & $\begin{array}{l}\text { 'aneurysm*':ab,ti AND ('concomitant':ab,ti OR 'prevalence':ab,ti OR 'co prevalence':ab,ti OR } \\
\text { 'coexistent':ab,ti OR 'concordant':ab,ti OR 'conjoined':ab,ti OR 'multianeurysm':ab,ti OR } \\
\text { 'synchron*':ab,ti OR 'accessor*':ab,ti) }\end{array}$ & 6,646 \\
\hline Cochrane & 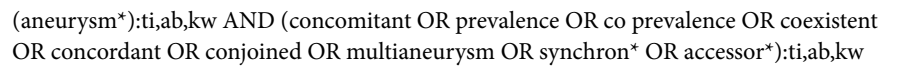 & 272 \\
\hline
\end{tabular}


Supplemental Table II. Excluded references after full text screening.

\begin{tabular}{|c|c|c|}
\hline Exclusion & Refe & ence \\
\hline \multirow[t]{5}{*}{ No full text available } & 1. & $\begin{array}{l}\text { Batt M, et al. (1985). Popliteal aneurysm. Personal experience of } 119 \text { cases. Journal de } \\
\text { Chirurgie 122: 319-325. }\end{array}$ \\
\hline & 2. & $\begin{array}{l}\text { Cole CW, et al. (1989). Popliteal aneurysms: An index of generalized vascular disease. } \\
\text { Canadian Journal of Surgery 32: 65-68. }\end{array}$ \\
\hline & 3. & $\begin{array}{l}\text { Mangiante EC, et al. (1984). Popliteal aneurysms. A clinical appraisal. American Surgeon } \\
\text { 50: 469-472. }\end{array}$ \\
\hline & 4. & $\begin{array}{l}\text { Mellière D, et al. (1998). Combined abdominal aortic aneurysm and internal iliac arterial } \\
\text { aneurysm. Journal des maladies vasculaires 23: } 342-348 \text {. }\end{array}$ \\
\hline & 5. & $\begin{array}{l}\text { Pasternak J, et al. (2015). Symptomatic popliteal artery aneurysms as limb threatening } \\
\text { disease. Italian Journal of Vascular and Endovascular Surgery 22: 197-201. }\end{array}$ \\
\hline \multirow{10}{*}{$\begin{array}{l}\text { Other study } \\
\text { purpose: did not } \\
\text { investigate } \\
\text { co-prevalence in two } \\
\text { different arteries }\end{array}$} & 6. & $\begin{array}{l}\text { Casimiro MV, et al. (2004). A comparison of risk factors in the etiology of mirror and } \\
\text { nonmirror multiple intracranial aneurysms. Surgical Neurology 61: 541-545. }\end{array}$ \\
\hline & & $\begin{array}{l}\text { Cousins R, et al. (2017). Determining patient risk factors associated with accelerated } \\
\text { growth of popliteal artery aneurysms. Journal of vascular surgery 65: e15-e16. }\end{array}$ \\
\hline & & $\begin{array}{l}\text { Huang ZQ, et al. (2017). Risk factors analysis of mirror aneurysms: A multi-center } \\
\text { retrospective study based on clinical and demographic profile of patients. European } \\
\text { Journal of Radiology 96: } 80-84 \text {. }\end{array}$ \\
\hline & & $\begin{array}{l}\text { Lai HP, et al. (2009). Size, location, and multiplicity of ruptured intracranial aneurysms in } \\
\text { the Hong Kong Chinese population with subarachnoid haemorrhage. Hong Kong Medical } \\
\text { Journal 15: 262-266. }\end{array}$ \\
\hline & & $\begin{array}{l}\text { Roethlisberger M, et al. (2018). Predictors of Occurrence and Anatomic Distribution of } \\
\text { Multiple Aneurysms in Patients with Aneurysmal Subarachnoid Hemorrhage. World } \\
\text { Neurosurg 111: e199-e205. }\end{array}$ \\
\hline & & $\begin{array}{l}\text { Wang MY, et al. (2000). Ethnic differences in the distribution of posterior circulation } \\
\text { aneurysms. Journal of Stroke and Cerebrovascular Diseases 9: 31-35. }\end{array}$ \\
\hline & & $\begin{array}{l}\text { Chang SW, et al. (2010). Treatment of distal posterior cerebral artery aneurysms: A critical } \\
\text { appraisal of the occipital artery-to-posterior cerebral artery bypass. Neurosurgery } 67 \text { : } \\
\text { 16-25. }\end{array}$ \\
\hline & & $\begin{array}{l}\text { Nordanstig J, et al. (2014). National experience with extracranial carotid artery aneurysms: } \\
\text { Epidemiology, surgical treatment strategy, and treatment outcome. Annals of vascular } \\
\text { surgery 28: 882-886. }\end{array}$ \\
\hline & & $\begin{array}{l}\text { Ogengo JA, et al. (2010). Pattern of femoro-popliteal aneurysms in an African population. } \\
\text { Journal Vascular Brasileiro 9: 36-39. }\end{array}$ \\
\hline & & $\begin{array}{l}\text { Park S, et al. (2014). Incidental Saccular Aneurysms on Head MR Angiography: } 5 \text { Years' } \\
\text { Experience at a Single Large-Volume Center. J Stroke 16: 189-194. }\end{array}$ \\
\hline \multirow[t]{2}{*}{ Book chapter } & & $\begin{array}{l}\text { Dawson J, et al. (2013). Update on aneurysm disease: Current insights and controversies. } \\
\text { peripheral aneurysms: When to intervene - Is rupture really a danger? Progress in } \\
\text { Cardiovascular Diseases 56: 26-35. }\end{array}$ \\
\hline & & $\begin{array}{l}\text { Hultgren R. (2013). Abdominal aortic aneurysms - Gender aspects on prevalence, } \\
\text { treatment, and concurrent aneurysms. Thoracic and Cardiovascular Surgeon 61: 15-21. }\end{array}$ \\
\hline \multirow[t]{2}{*}{$\begin{array}{l}\text { Describing the same } \\
\text { patient population }\end{array}$} & & $\begin{array}{l}\text { Larsson E, et al. (2011) High frequency of thoracic aneurysms in patients with abdominal } \\
\text { aortic aneurysms. Ann Surg. 2011;253:180-4. }\end{array}$ \\
\hline & & $\begin{array}{l}\text { Pourier VEC, et al (2017) Prevalence of extracranial carotid artery aneurysms in patients } \\
\text { with an intracranial aneurysm. PLoS ONE 12(11):e0187479. }\end{array}$ \\
\hline $\begin{array}{l}\text { No separate data of } \\
\text { dissection versus } \\
\text { aneurysm }\end{array}$ & & $\begin{array}{l}\text { Song J, et al. (2020) Prevalence of intracranial aneurysms in patients with systemic vessel } \\
\text { aneurysms, a nationwide cohort study. Stroke 2020;51:115-120. }\end{array}$ \\
\hline
\end{tabular}


Supplemental Table III. Scoring definitions for quality assessment table (Supplemental Table III), modified Risk of Bias prevalence tool by Hoy et al. ${ }^{12}$

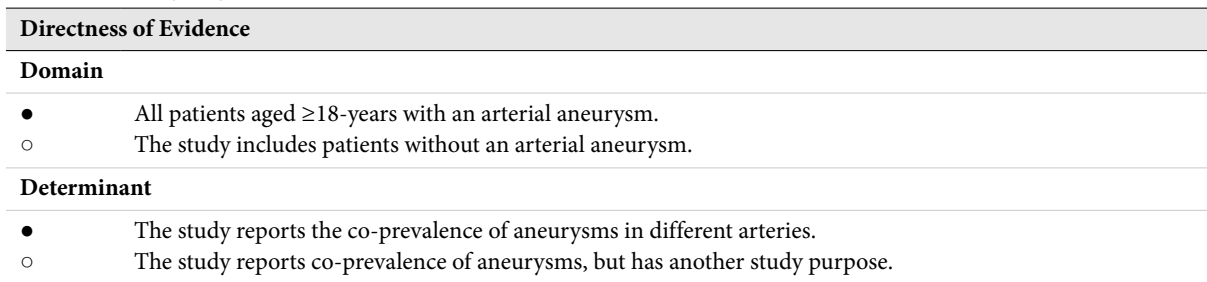

\section{Co-prevalence}

- The study describes a co-prevalence among $\geq 2$ aneurysms in at least 2 different types of arteries.

$\odot \quad$ The study describes a co-prevalence of extended aneurysms in 2 different types of arteries (e.g. aortoiliac).

- The study describes a co-prevalence among $\geq 2$ aneurysms in the same artery.

\section{Putative risk factors}

- $\quad$ Adjusted putative clinical risk factors for co-prevalence of aneurysms are described.

$\odot \quad$ Unadjusted / univariate risk factors are described.

○ No risk factors are described.

\section{Risk of Bias}

Sampling frame

- $\quad$ The sampling frame was a true or close representation of patients with $\geq 1$ aneurysms.

- The sampling frame was not a true or close representation of patients with $\geq 1$ aneurysms.

\section{Random selection and in-/exclusion}

- $\quad$ Some form of random selection was used to select the patient sample (e.g. screening program, hospital database); in- and exclusion criteria were specified.

$\odot \quad$ Random selection was absent, OR in- and exclusion criteria were absent.

$\circ \quad$ The selection of patients, AND in- and exclusion criteria were not reported.

\section{Aneurysm definition}

- $\quad$ Both aneurysms are defined, either as ratio relative to the non-affected (contralateral if applicable) part of the artery, or as absolute values.

$\odot \quad$ At least one aneurysm is defined.

- Aneurysm definitions are not described.

\section{Imaging modality}

- $\quad$ The type of chosen imaging scanner was reported and reasoned in the study period.

$\circ \quad$ No detailed imaging modality descriptives are reported or reasoned.

\section{Consistent data collection}

- $\quad$ The same mode of imaging modality was used for all subjects.

$\circ \quad$ Different imaging modalities were used to screen subjects.

\section{Reproducibility}

- $\quad \geq 2$ independent observers assessed aneurysm co-prevalence and reported reliability and agreement.

$\odot \quad \geq 2$ independent observers assessed aneurysm co-prevalence without reported reproducibility measures.

$\circ \quad$ A single observer assessed aneurysm co-prevalence, or no observer information was reported.

\section{Incomplete patient data}

- $\quad$ The study describes a percentage of $<15 \%$ missing patient data.

$\circ \quad$ The study describes a percentage of $\geq 15 \%$ missing patient data.

$\mathrm{n} / \mathrm{r} \quad$ The study does not report any information concerning missing data.

\section{Correct calculation of co-prevalence and $95 \%$ confidence interval}

- $\quad$ The paper presented an adequate numerator and denominator for aneurysm co-prevalence, and absolute numbers or $95 \%$ confidence intervals were reported.

$\circ \quad$ No correct calculation of co-prevalence, and absolute numbers and $95 \%$ confidence interval were reported. 
Supplemental Table IV. Overview of the 32 included articles.

\begin{tabular}{|c|c|c|c|c|c|c|}
\hline \multirow{2}{*}{$\begin{array}{l}\text { Reference } \\
1 .\end{array}$} & \multirow{2}{*}{\begin{tabular}{|l|} 
PubMed ID \\
31327605
\end{tabular}} & \multirow{2}{*}{$\begin{array}{l}\text { First Author } \\
\text { Laukka }\end{array}$} & \multirow{2}{*}{$\begin{array}{l}\text { Year } \\
2019\end{array}$} & \multirow{2}{*}{$\begin{array}{l}\text { Study population } \\
\text { Finland }\end{array}$} & \multicolumn{2}{|c|}{$\begin{array}{l}\text { Risk of Bias } \\
\text { (score) }\end{array}$} \\
\hline & & & & & M & $(4.5)$ \\
\hline 2. & 25428688 & Goyal & 2015 & Missouri (US) & $\mathrm{H}$ & (3) \\
\hline 3. & 31412341 & Malhotra & 2019 & New Haven (US) & M & $(4)$ \\
\hline 4. & 20102960 & Kuzmik & 2010 & New York (US) & $\mathrm{H}$ & $(2.5)$ \\
\hline 5. & 31556002 & Erben & 2019 & Florida (US) & M & $(5.5)$ \\
\hline 6. & 17273890 & Miyazawa & 2007 & Japan & M & $(5)$ \\
\hline 7. & 31538843 & Kurtelius & 2019 & Finland & M & $(4.5)$ \\
\hline 8. & 27609114 & Lee & 2017 & South Korea & $\mathrm{L}$ & $(6)$ \\
\hline 9. & 27256853 & Rouchaud & 2016 & Minnesota (US) & M & (5) \\
\hline 10. & 25991415 & Shin & 2015 & South Korea & $\mathrm{H}$ & $(3.5)$ \\
\hline 11. & 31200039 & Hill & 2019 & Michigan (US) & $\mathrm{H}$ & $(2.5)$ \\
\hline 12. & 31971973 & van Laarhoven & 2020 & Finland + the Netherlands & $\mathrm{L}$ & $(6)$ \\
\hline 13. & 31310617 & Endo & 2019 & Japan & $\mathrm{L}$ & $(6)$ \\
\hline 14. & 8417547 & Stajduhar & 1993 & Washington D.C. (US) & M & $(4.5)$ \\
\hline 15. & 25379626 & Balderston & 2015 & Philadelphia (US) & $\mathrm{L}$ & $(6)$ \\
\hline 16. & 26602411 & DeFreitas & 2016 & Michigan (US) & $\mathrm{L}$ & $(6)$ \\
\hline 17. & 31783113 & Dombrowski & 2019 & Michigan (US) & M & $(5)$ \\
\hline 18. & 29935861 & Wallinder & 2018 & Sweden & $\mathrm{H}$ & (3) \\
\hline 19. & 22944566 & Hultgren & 2012 & Sweden & $\mathrm{L}$ & (6) \\
\hline 20. & 22727846 & Chaer & 2012 & Pittsburgh (US) & M & $(4)$ \\
\hline 21. & 28551718 & Chapman & 2017 & Michigan (US) & $\mathrm{L}$ & $(6)$ \\
\hline 22. & 31815625 & Hohneck & 2019 & Germany & $\mathrm{L}$ & (6) \\
\hline 23. & 30721728 & Studzińska & 2019 & Poland & $\mathrm{L}$ & $(7.5)$ \\
\hline 24. & 9587341 & Armon & 1998 & United Kingdom & M & $(5.5)$ \\
\hline 25. & 5044555 & Dent & 1972 & Michigan (US) & $\mathrm{H}$ & (3) \\
\hline 26. & 28010870 & Laine & 2017 & MultiCenter (EU + AUS) & M & $(5)$ \\
\hline 27. & 7362460 & Graham & 1980 & Michigan (US) & $\mathrm{H}$ & (3) \\
\hline 28. & 31757587 & Cervin & 2020 & Sweden & M & $(4.5)$ \\
\hline 29. & 27216869 & Tuveson & 2016 & Sweden & $\mathrm{L}$ & $(6)$ \\
\hline 30. & 10805875 & Diwan & 2000 & Michigan (US) & $\mathrm{L}$ & $(6.5)$ \\
\hline 31. & 29126647 & Ravn & 2017 & Sweden & $\mathrm{H}$ & $(1.5)$ \\
\hline 32. & 18306151 & Ravn & 2008 & Sweden & M & (5.5) \\
\hline
\end{tabular}

Abbreviations: US: United States, EU: Europe, AUS: Australia, H: high, M: moderate, L: low.

\section{Supplemental Material II is available as online table.}





\section{Chapter 6}

\section{Polygenic susceptibility of aortic aneurysms associates to the diameter of the aneurysm sac: the Aneurysm-Express biobank cohort}

Sci Rep 2019;27:19844

C.J.H.C.M. van Laarhoven

J. van Setten

J.A. van Herwaarden

G.A. Pasterkamp

D.P.V. de Kleijn

G.J. de Borst

S.W. van der Laan 


\section{Abstract}

\section{Background and purpose}

Recent genome-wide association studies (GWAS) have discovered ten genetic risk variants for abdominal aortic aneurysms (AAA). To what extent these genetic variants contribute to the pathology of aneurysms is yet unknown.

\section{Methods}

The present study aims to investigate whether genetic risk variants are associated with three clinical features: diameter of aneurysm sac, type of artery and aneurysm relatedsymptoms in aortic and peripheral aneurysm patients. Aneurysm tissue of 415 patients included in the Aneurysm-Express biobank was used. A best-fit polygenic risk score (PRS) based on previous GWAS effect estimates was modeled for each clinical phenotype.

\section{Results}

The best-fit PRS (including 272 variants at $P_{T}=0.01015$ ) showed a significant correlation with aneurysm diameter $\left(\mathrm{R}^{2}=0.019, p=0.001\right)$. No polygenic association was found with clinical symptoms or artery type. In addition, the ten genome-wide significant risk variants for AAA were tested individually, but no associations were observed with any of the clinical phenotypes. All models were corrected for confounders and data was normalized.

\section{Conclusion}

In conclusion, a weighted PRS of AAA susceptibility explained $1.9 \%$ of the phenotypic variation $(p=0.001)$ in diameter in aneurysm patients. Given our limited sample size, future biobank collaborations need to confirm a potential causal role of susceptibility variants on aneurysmal disease initiation and progression. 


\section{Introduction}

Abdominal aortic aneurysm disease (AAA) is a vascular pathology, affecting in particular elderly Western men ${ }^{1-3}$. Besides sex and smoking, a positive family history is a known predisposing factor for occurrence of the disease $e^{4,5}$, indicative of a strong heritable component to AAA.

In the past decade, genome-wide association studies (GWAS) have uncovered ten common genetic variants, i.e. single-nucleotide polymorphisms (SNPs), associated to AAA susceptibility ${ }^{6-12}$. While these GWAS point out part of the genetic underpinnings of AAA, the extent to which these variants influence the clinical presentation, whether these loci are specific for a vascular bed, or how these loci contribute to the pathology of the disease is still largely unknown.

Previous literature has shown many complex traits to be polygenic in origin, comprising small effects of hundreds or even thousands of common variants that in aggregate explain a substantial proportion of trait susceptibility and heritability ${ }^{13-15}$. For example, the International Schizophrenia Consortium ${ }^{16}$ summarized weighted genetic effects ${ }^{13}$ across nominally associated loci at increasingly liberal $p$-value thresholds into polygenic risk scores (PRS), and correlated PRS to disease susceptibility, demonstrating that a PRS can provide a reliable genetic indicator for clinical outcome.

In the present study, we investigated whether AAA susceptibility variants in aggregate are associated with three clinical phenotypes (maximum diameter of aneurysm sac, aneurysm related symptoms, and type of artery) within the Aneurysm-Express biobank study ${ }^{17}$. These phenotypes encompass interventions and are therefore clinically relevant. First, we constructed a weighted PRS based on summary level GWAS data for AAA ${ }^{12}$ using increasingly liberal $p$-value thresholds and modeled a best fit PRS. Secondly, we tested the genome-wide significant risk SNPs for association with the selected clinical phenotypes in our cohort of aortic and peripheral aneurysm patients with clinically manifested disease. Our results show that higher PRS associates to larger aneurysm diameter, but no association was found with clinical symptoms or type of artery.

\section{Methods}

\section{Aneurysm-Express biobank study}

The Aneurysm-Express is a biobank study that contains aneurysm sac tissue from patients undergoing open surgical repair of arterial aneurysms. The study design has been published previously ${ }^{17}$. Ethical approval for this study (TME/C-01.18) was provided by the Medical Research Ethics Committee of University Medical Center Utrecht, Utrecht, The Netherlands on 10 April 2002, and all research was conducted according to the 
principles of the Declaration of Helsinki (59 ${ }^{\text {th }}$ amendment, Seoul 2008) and in accordance with the Dutch Medical Research Involving Human Subjects Act (WMO). Patients were operated in two different Dutch hospitals and all participants gave informed consent. For the present study we used clinical information from consecutive patients who were included between 2003 and 2013. The indications to perform open repair were based on at that time current guidelines ${ }^{3}$. Patients with arterial aneurysms caused by dissection, connective tissue disorders, and mycotic aneurysms or re-operated patients were excluded from this study. Risk factors and demographic data were obtained from clinical records and questionnaires at time of recruitment.

\section{Genotyping, quality control, and imputation}

DNA of 503 patients in Aneurysm-Express biobank study was extracted from whole blood EDTA or (when no blood was available) aneurysm tissue samples following standardized in-house validated protocols for the Aneurysm-Express Genomics Study (AAAGS). Samples were sent for genotyping at the Genomic Analysis Center of the Helmholtz Zentrum Münich (Germany) according to OECD standards under study number M00750 using the Illumina HumanCoreExome BeadArray v1.1 (Illumina Inc., www.illumina.com).

Genotype calling was done with the GenomeStudio V2011.1 software and the Genotyping module version 1.9.4 using the original Illumina cluster and manifest files (humancoreexome-12v1-1_a.egt and HumanCoreExome-12- v1-1-C.bpm). The GenCall score cutoff was 0.15 as recommended by Illumina. The average call rate of all samples was $99.55 \%$ across 542,585 variants.

Subsequently, community standard quality control (QC) procedures were applied ${ }^{18}$ to obtain high quality data. Samples with low average genotype calling and sex discrepancies (compared to the clinical data available) based on GenomeStudio metrics were excluded. The data was filtered on 1) individual (sample) call rate $>97 \%, 2$ ) SNP call rate $>96 \%, 3$ ) average heterozygosity rate \pm 2.5 s.d., 4) relatedness (pi-hat $>0.20$ ), 5) Hardy-Weinberg Equilibrium (HWE $\mathrm{p}<1.0 \times 10^{-6}$ ), and 6) population stratification excluding nonEuropeans (based on $1000 \mathrm{G}$ phase 3$)^{19}$. $\mathrm{zCall}^{20}$ was used to call missing exome-variants after QC. After QC and resulted in 478 samples and 541,569 variants (call rate $=99.99 \%$ ) remained and were used for imputation. Autosomal missing genotypes were imputed based on phased integrated data from 1000 Genomes (phase 3, version 5) and Genome of the Netherlands $v 5^{21}$ using IMPUTE2 (v2.3.0 $)^{22}$ after pre-phasing genotyped data with SHAPEIT2 (v2.644) $)^{23}$.

\section{Polygenic Risk Score (PRS)}

The PRSice software ${ }^{13}$ was used for creating the best fit PRS per phenotype by comparing scores across a range of different $p$-value thresholds. In short, PRSice calculates weighted 
PRS based on the effect estimates reported in the meta-analysis of GWAS ${ }^{12,24}$. To this end, variants are pruned based on the linkage disequilibrium $\left(\mathrm{r}^{2}<0.1 \text {, clump, range } 500 \mathrm{~kb}\right)^{20}$ as observed in AAAGS for bins of increasingly liberal $p$-value thresholds $\left(p_{T}\right.$, see Supplemental Table I), and preferentially retaining variants with lower $p$-values as reported by the GWAS ${ }^{12}$ (similar to the --clump algorithm in $\mathrm{PLINK}^{25}$ ). Odds ratios were natural log transformed to betas $(\beta)$, we only included variants with MAF $>0.05$ and imputation quality info-score $>0.8$. In order to verify the predictive value of the PRS of the AAA-GWAS, we additionally modeled a PRS of summary statistics of the attention deficit hyperactivity disorder (ADHD) GWAS unrelated to $\mathrm{AAA}^{24}$, and tested this additional polygenic score for association with the selected clinical parameters in our study cohort.

\section{Individual SNP analysis}

We selected ten SNPs that were identified in a recent GWAS meta-analysis for AAA ${ }^{12}$. These SNPs were tested for association with the clinical features separately. For the SNP lookup we used GWASToolKit (https:/github.com/swvanderlaan/GWASToolKit, doi: 10.5281/zenodo.997862), which is a collection of scripts to execute SNPTEST v2.5.3 ${ }^{26}$ analyses. Given the limited sample size, we calculated the expected power for this analysis $^{27}$. In case of a risk allele frequency of $<20 \%$, and estimated OR of 1.10 , the resulting power is $\pm 80 \%$ (Supplemental Figure I).

\section{Primary endpoints}

The primary outcomes or phenotypes were artery type, symptoms, and diameter of the aneurysm sac. Artery type was defined as either aortic, or peripheral (iliac, femoral, popliteal, and carotid). Symptom status was defined as asymptomatic, or any aneurysm related symptoms like thromboembolic events, local pain or swelling, or rupture of the aneurysm sac. Maximum diameter was measured by experienced radiologists at time of inclusion, by using the double oblique plane of computed tomography angiography.

\section{Statistical analysis}

Baseline characteristics were compared between patients with upper $20^{\text {th }}$ percentile and lower $80^{\text {th }}$ percentile of the PRS (see Supplemental Table IV). For continuous baseline variables, the Student's T-test was used for normally distributed variables and MannWhitney $U$ test for non-normally distributed variables. Categorical baseline variables were tested by the Chi-squared test for equal distribution among both groups. PRS and individual SNP analyses were performed using linear and logistic regression models where appropriate, adjusted for sex, age, ancestral background using four principal components, smoking status, and diameter of the aneurysm sac or artery type if applicable. Nagelkerke's $r^{2}$ was used as a metric of the variance explained by the polygenic model. Statistical analyses were 
conducted using SPSS v25.0 (IBM Corp. Released 2017. IBM SPSS Statistics for Windows, Version 25.0. Armonk, NY: IBM Corp.), PRSice v2.1.4. ${ }^{13}$, R v3.4.0 (R Core Team (2017), R: A language and environment for statistical computing. R Foundation for Statistical Computing, Vienna, Austria. URL http://www.R-project.org).

\section{Results}

In the present study a total of 415 aortic and peripheral aneurysm patients from the Aneurysm-Express biobank were included. The average age was $69 \pm 8.1$ years, the majority (85\%) male, and 349 (84\%) patients were treated for an AAA (Table 1).

We used summary statistics from the largest meta-GWAS for AAA so far ${ }^{12}$ to calculate weighted polygenic scores at increasingly liberal $p$-value thresholds $\left(p_{T}\right.$, Supplemental Table I), and correlated these to artery type, symptoms, and diameter of the aneurysm sac (Figure 1A-C). A PRS including 272 variants at $p_{T}$ of 0.01015 explained the largest proportion in diameter $\left(\mathrm{R}^{2}=0.019, p=0.001\right.$, Supplemental Table II). Diameter per 10.0 millimeter and average PRS is visualized in Figure 2, showing higher PRS in large aneurysms. Distributions of the AAA-PRS for diameter were comparable between artery types (Kruskal-Wallis test $p$-value $=0.135$, Supplemental Figure II). No association was found for artery type and symptom status (Supplemental Table II). We verified all AAAPRS findings with another unrelated PRS derived from a GWAS on $\mathrm{ADHD}^{24}$, and correlated these with the selected clinical parameters in our study cohort (Figure 1D-F). No associations with the ADHD-PRS were observed (Figure 1D-F, Supplementary Table III). Clinical characteristics of patients within the upper $20^{\text {th }}$ and lower $80^{\text {th }}$ percentile of the PRS distribution for diameter is shown in Supplemental Table IV.

Next, we tested ten known AAA SNPs for association with diameter of the aneurysm sac (Table 2), artery type and symptom status (Supplemental Table V). For diameter, a nominal association was found with rs1466535 (12q13.3, LRP1, $p=0.013)$. For rs602633 (1p13.3, PSRC1- CELSR2-SORT1), rs1795061 (1q32.3, SMYD2), rs2836411 (21q22.2, $E R G)$ and rs1466535 a concordant effect direction was observed. For six risk variants, the effect direction was discordant with the GWAS results.

\section{Discussion}

In this study, we investigated the association of genetic susceptibility variants with clinical phenotypes within a biobank consisting of surgically treated aortic and peripheral aneurysms. Our results demonstrate that polygenic scores of AAA susceptibility are associated with diameter of the aneurysm sac in patients with aneurysms in different vascular beds. 
Table 1. Baseline characteristics of included study patients.

\begin{tabular}{|c|c|c|c|c|c|c|c|c|c|c|}
\hline \multirow[b]{2}{*}{ Male } & \multicolumn{2}{|c|}{$\begin{array}{c}\text { AAA } \\
\mathrm{n}=349\end{array}$} & \multicolumn{2}{|c|}{$\begin{array}{c}\text { Iliac } \\
n=13\end{array}$} & \multicolumn{2}{|c|}{$\begin{array}{c}\text { Femoral } \\
n=9\end{array}$} & \multicolumn{2}{|c|}{$\begin{array}{c}\text { Popliteal } \\
\mathbf{n}=35\end{array}$} & \multicolumn{2}{|c|}{$\begin{array}{c}\text { Carotid } \\
n=9\end{array}$} \\
\hline & 292 & $84 \%$ & 13 & $100 \%$ & 8 & $100 \%$ & 34 & $97 \%$ & 4 & $44 \%$ \\
\hline $\begin{array}{l}\text { Age at surgery } \\
\text { in years (range) }\end{array}$ & 70 & $\begin{array}{l}7.1 \\
(48-89)\end{array}$ & 65 & $\begin{array}{l}11.0 \\
(48-86)\end{array}$ & 67 & $\begin{array}{l}11.2 \\
(49-87)\end{array}$ & 66 & $\begin{array}{l}10.0 \\
(45-83)\end{array}$ & 62 & $\begin{array}{l}9.7 \\
(46-75)\end{array}$ \\
\hline \multicolumn{11}{|l|}{ Aneurysm shape } \\
\hline saccular & 16 & $5 \%$ & 0 & - & 0 & - & 1 & $3 \%$ & 3 & $33 \%$ \\
\hline fusiform & 332 & $95 \%$ & 13 & $100 \%$ & 9 & $100 \%$ & 34 & $97 \%$ & 6 & $67 \%$ \\
\hline $\begin{array}{l}\text { Reported aneurysm } \\
\text { diameter }(\mathbf{m m})\end{array}$ & 64 & $\begin{array}{l}13.9 \\
(31-118)\end{array}$ & 46 & $\begin{array}{l}12.4 \\
(25-70)\end{array}$ & 44 & $\begin{array}{l}25.5 \\
(24-100)\end{array}$ & 35 & $\begin{array}{l}20.0 \\
(11-105)\end{array}$ & 23 & $\begin{array}{l}10.2 \\
(12-38)\end{array}$ \\
\hline \multicolumn{11}{|l|}{ Symptoms } \\
\hline Ruptured & 28 & $8 \%$ & 1 & $8 \%$ & 0 & - & 2 & $6 \%$ & 0 & - \\
\hline $\begin{array}{l}\text { Any aneurysm } \\
\text { related symptom }\end{array}$ & 82 & $24 \%$ & 4 & $31 \%$ & 4 & $44 \%$ & 19 & $54 \%$ & 6 & $68 \%$ \\
\hline Asymptomatic & 237 & $68 \%$ & 7 & $54 \%$ & 5 & $56 \%$ & 14 & $40 \%$ & 3 & $33 \%$ \\
\hline Hypertension & 275 & $79 \%$ & 7 & $54 \%$ & 4 & $44 \%$ & 22 & $63 \%$ & 4 & $44 \%$ \\
\hline Diabetes & 58 & $17 \%$ & 1 & $8 \%$ & 1 & $11 \%$ & 4 & $11 \%$ & 1 & $11 \%$ \\
\hline Statin use & 237 & $68 \%$ & 7 & $54 \%$ & 3 & $33 \%$ & 19 & $54 \%$ & 6 & $67 \%$ \\
\hline BMI & 25.9 & 4.1 & 26.4 & 5.2 & 27.7 & 4.6 & 27.2 & 3.6 & 23.7 & 2.6 \\
\hline Smoking & 126 & $36 \%$ & 6 & $46 \%$ & 2 & $22 \%$ & 12 & $34 \%$ & 0 & - \\
\hline
\end{tabular}

Data are given as numbers (percentage) or mean (standard deviation).

Abbreviations: $\mathrm{AAA}=$ abdominal aortic aneurysm, $\mathrm{mm}=$ millimeter, $\mathrm{BMI}=$ body mass index .

The clinical translation of genetic research has been a topic of interest for the last decades. For rare diseases like Marfan or Ehlers-Danlos syndrome, genetic clinical utility has been proven and applied ${ }^{28}$, but for more common complex diseases like AAA, the interpretation and translation of large-scale genetic studies is challenging at best ${ }^{29}$. GWAS-derived association studies were mainly investigated in intracranial and abdominal aneurysm patients $^{30-35}$. The majority of these studies used a genetic risk score (GRS) based on the index SNPs from GWAS to study additional genetic predicting value to models consisting of demographic characteristics and health parameters. A previous study using a GRS including 4 variants showed that a high GRS was associated with aneurysm growth rate independent of baseline abdominal aortic size $e^{36}$. Recently, a PRS approach, which includes millions of nominally associated SNPs, has proved clinical utility in atherosclerosis, statin therapy, and breast cancer ${ }^{37-39}$. A polygenic approach can optimize power by using a liberal $p$-value threshold, enforced by the evidence that additive weak effects of nominally 

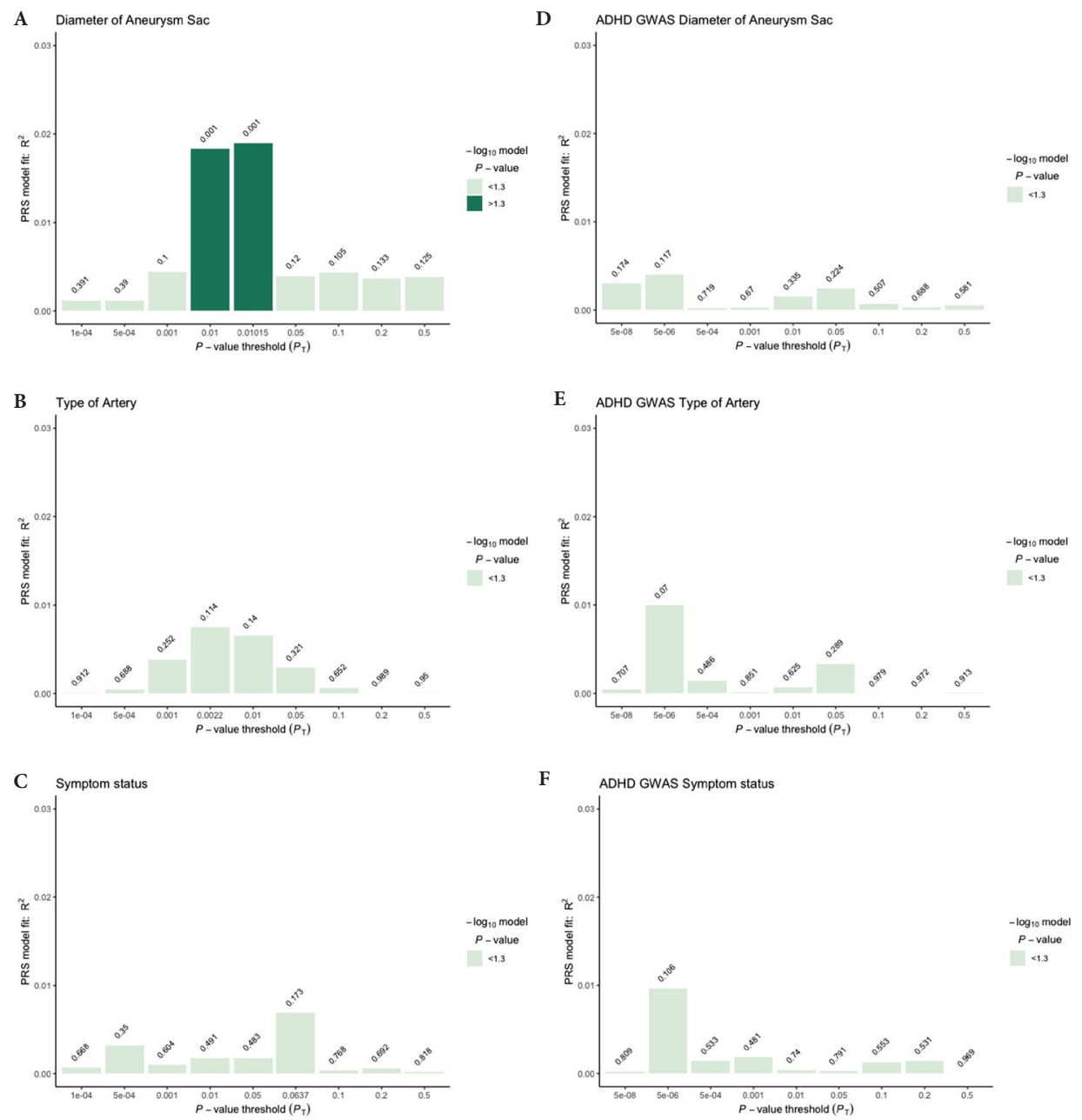

Figure 1. PRSice ${ }^{13}$ generated weighted model for: A Diameter of the aneurysm sac, B Type of artery, and C Symptom status. Models are summarized in Supplementary Table 2\&3. D-E-F indicate unrelated PRS derived from ADHD GWAS summary statistics ${ }^{24}$, showing no association with any of the selected clinical phenotypes in the Aneurysm-Express biobank cohort. Abbreviations: $\mathrm{PRS}=$ polygenic risk score, $\mathrm{ADHD}=$ attention deficit hyperactivity disorder, GWAS = genome-wide association study.

associated common variants explain part of the heritability of, and susceptibility to common complex diseases ${ }^{14,15}$. Our study uses a polygenic risk approach within a cohort comprising both aortic and peripheral aneurysm patients and shows that a high polygenic risk of AAA is associated to larger diameter of the aneurysm sac, although clinical parameters of patients within the upper $20^{\text {th }}$ and lower $80^{\text {th }}$ percentile of the PRS distribution were not distinctive in post-operative outcome or inflammatory status of the aneurysm (Supplemental Table IV). 
Table 2. Individual SNP analysis of AAA associated SNPs reported by GWAS and the association results for diameter of the aneurysm sac (in millimeters).

\begin{tabular}{|c|c|c|c|c|c|c|c|c|c|c|c|}
\hline \multicolumn{8}{|c|}{ Reported by literature } & \multicolumn{4}{|c|}{ This study } \\
\hline SNP & Chr & BP & $\begin{array}{c}\text { Near(est) } \\
\text { gene(s) }\end{array}$ & Alleles & EAF & $\beta^{\mathrm{a}}$ & $p$ & EAF & $\beta$ & SE & $p$ \\
\hline rs602633 & 1 & 109821511 & $\begin{array}{c}\text { PSRC1- } \\
\text { CELSR2-SORT1 }\end{array}$ & $\mathrm{T}^{*}-\mathrm{G}$ & 0.199 & -0.129 & $\begin{array}{c}6.58 \\
\times 10^{-9}\end{array}$ & 0.208 & -2.017 & 1.238 & 0.10 \\
\hline rs4129267 & 1 & 154426264 & $I L 6 R$ & $\mathrm{~T}^{*}-\mathrm{C}$ & 0.370 & -0.132 & $\begin{array}{c}4.76 \\
\times 10^{-13}\end{array}$ & 0.355 & 1.422 & 1.073 & 0.186 \\
\hline rs1795061 & 1 & 214409280 & SMYD2 & $\mathrm{T}^{*}-\mathrm{C}$ & 0.337 & 0.123 & $\begin{array}{c}8.80 \\
\times 10^{-11}\end{array}$ & 0.307 & 0.490 & 1.054 & 0.642 \\
\hline rs10757274 & 9 & 22096055 & $\begin{array}{c}\text { CDKN2BAS1/ } \\
\text { ANRIL }\end{array}$ & $A^{*}-G$ & 0.462 & -0.216 & $\begin{array}{c}1.54 \\
\times 10^{-33}\end{array}$ & 0.504 & 0.319 & 1.264 & 0.752 \\
\hline rs10985349 & 9 & 124425243 & $D A B 2 I P$ & $\mathrm{~T}^{*}-\mathrm{C}$ & 0.195 & 0.158 & $\begin{array}{c}2.40 \\
\mathrm{x} 10^{-11}\end{array}$ & 0.200 & -0.445 & 1.009 & 0.725 \\
\hline rs1466535 & 12 & 57534470 & $L R P 1$ & $G^{*}-A$ & 0.679 & $0.199^{\mathrm{b}}$ & $\begin{array}{r}9.99 \\
\times 10^{-7}\end{array}$ & 0.655 & 2.554 & 1.022 & 0.013 \\
\hline rs9316871 & 13 & 22861921 & LINC00540 & $A^{*}-G$ & 0.201 & -0.136 & $\begin{array}{c}4.75 \\
\times 10^{-10}\end{array}$ & 0.796 & 0.675 & 1.272 & 0.596 \\
\hline rs6511720 & 19 & 11202306 & $L D L R$ & $\mathrm{~T}^{*}-\mathrm{G}$ & 0.096 & -0.218 & $\begin{array}{c}7.90 \\
\times 10^{-14}\end{array}$ & 0.094 & 2.612 & 1.755 & 0.138 \\
\hline rs3827066 & 20 & 44586023 & $\begin{array}{c}\text { PCIF1-ZNF335- } \\
M M P 9\end{array}$ & $\mathrm{~T}^{*}-\mathrm{C}$ & 0.179 & 0.201 & $\begin{array}{c}2.13 \\
\times 10^{-17}\end{array}$ & 0.167 & -0.900 & 1.323 & 0.496 \\
\hline rs2836411 & 21 & 39819830 & $E R G$ & $\mathrm{~T}^{*}-\mathrm{C}$ & 0.369 & 0.107 & $\begin{array}{c}5.80 \\
\times 10^{-9}\end{array}$ & 0.362 & 0.499 & 1.238 & 0.687 \\
\hline
\end{tabular}

Abbreviations: $\mathrm{SNP}=$ single nucleotide polymorphism, $\mathrm{AAA}=$ abdominal aortic aneurysm, $\mathrm{Chr}=$ chromosome, $\mathrm{BP}=$ base pair, $\mathrm{EAF}=$ effect allele frequency, $\beta=$ beta-coefficient, $\mathrm{SE}=$ standard error.

${ }^{\star}$ Effect allele, ${ }^{\text {a }} \beta$ converted from combined odds ratio's (discovery and validation phase) of summary statistics of Jones et al. $(2017)^{12}{ }^{b} \beta$ converted from discovery phase of summary statistics of Bown et al. (2011) ${ }^{7}$.

After correcting for multiple testing, no effects of individual SNPs were observed, and the majority of the effect directions in our analysis are discordant with the recent GWAS meta-analysis ${ }^{12}$. This is probably mainly due to limited size and power of both the published GWAS (4,972 cases and 99,858 controls) and our present study (Supplemental Figure II). Differences could also be explained by differences in inclusion criteria; the GWAS analysis is performed in patients with AAA $\geq 30 \mathrm{~mm}$, including smaller AAA that have been followed-up and were compared to healthy subjects ${ }^{29}$. In contrast, our cohort consists solely of symptomatic patients or patients with progressive aneurysmal disease that requires surgical treatment. It is arguably that GWAS identified loci may contribute particularly to initiation of aneurysmal disease, whilst they have discordant effects on disease progression and so other risk variants may have a more prominent role in further deterioration of the vessel wall. 


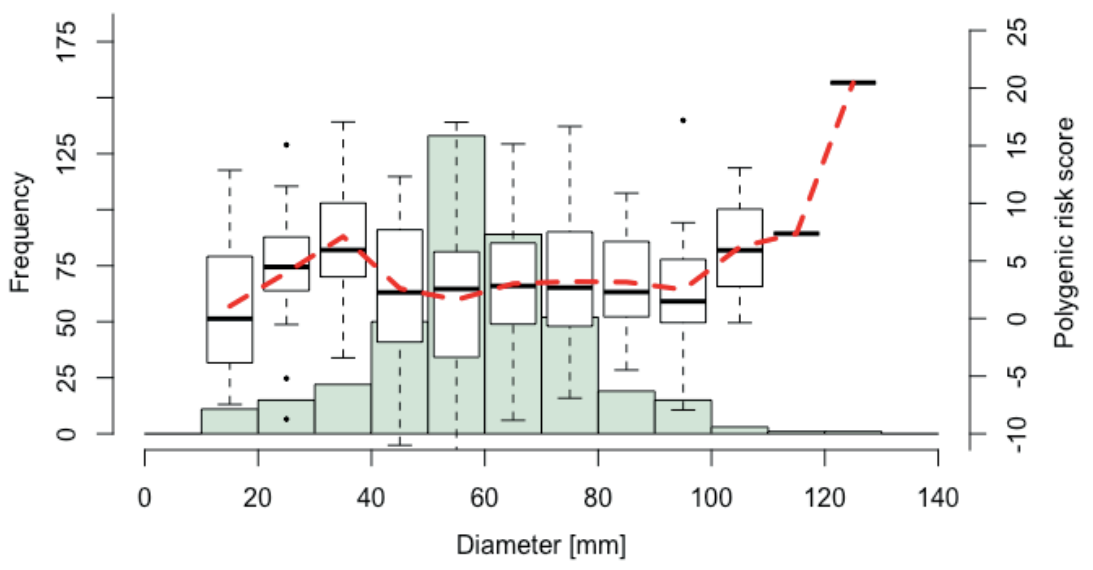

Figure 2. Distribution of maximum diameter of included aneurysms. Boxplots indicate PRS per $10.0 \mathrm{~mm}$ diameter, outliers are indicated as dots. Average PRS per diameter is indicated as red dashed line, showing an increase in average PRS from diameters $\geq 90.0 \mathrm{~mm}$. Abbreviations: $\mathrm{PRS}=$ polygenic risk score, $\mathrm{mm}=$ millimeter.

Whether aortic and intracranial aneurysms share genetic susceptibility, was previously investigated in a GWAS of four cohorts ${ }^{32}$. This study provides evidence that variants at 9p21, 18q11,15q21, and 2q33 are consistently associated with intracranial, thoracic aneurysms and AAA. Their analysis revealed no additional risk loci associated with joint aneurysms, presumably due to their limited sample size of 3,094 cases and 9,521 controls. The PRS distribution of diameter in the present study was comparable across artery types (Supplemental Figure II), this suggests overlapping genetic pathways between peripheral aneurysms and central aneurysms.

\section{Limitations}

The Aneurysm-Express biobank cohort is limited in size when compared to other biobanks, yet unique in its scope, given the availability of arterial aneurysm samples from the open arterial surgery era for analyses. Future studies should focus on biobank collaborations, enrichment, and harmonization to facilitate the in-depth scrutiny and replication of genetic susceptibility on aneurysm and AAA initiation and progression, as well as the exploration of sex differences. Our biobank is surgically driven, and measured diameters may cluster around surgical intervention thresholds (e.g. for AAA $\geq 55 \mathrm{~mm}$ ) limiting the generalizability, still we observed normally distributed diameters. Although the general pathology of aneurysms in various anatomical locations is similar, the initiating factors might be unique to the site. Artery type was used as composite covariate within the analyses, although individual aneurysm site factors might have confounded our results. 


\section{Conclusions}

In conclusion, we show that a weighted polygenic score of AAA susceptibility explained $1.9 \%$ of the phenotypic variation $(p=0.001)$ in aneurysm diameter in the AneurysmExpress biobank study. Future studies should focus on biobank collaborations, enrichment, and harmonization to assess potential impact of AAA susceptibility loci on aneurysmal disease initiation and progression. 


\section{References}

1. Singh K, et al. Prevalence of and risk factors for abdominal aortic aneurysms in a population based study: The Tromsø Study. Am J Epidemiol 2001; 154:236-244.

2. Lijftogt N, et al. Failure to Rescue e a Closer Look at Mortality Rates Has No Added Value for Hospital Comparisons but Is Useful for Team Quality Assessment in Abdominal Aortic Aneurysm Surgery in The Netherlands. Eur J Vasc Endovasc Surg 2018;56, 652e661.

3. Moll FL, et al. European Society for Vascular Surgery. Management of abdominal aortic aneurysms clinical practice guidelines of the European society for vascular surgery. Eur J Vasc Endovasc Surg 2011;41:S1-S58.

4. Wahlgren CM, et al. Genetic and environmental contributions to abdominal aortic aneurysm development in a twin population. J Vasc Surg. 2010;51:3-7.

5. Van den Luijtgaarden KM, et al. Risk of abdominal aortic aneurysm (AAA) among male and female relatives of AAA patients. Vascular Medicine 2017, 22;112-118.

6. Gretarsdottir $\mathrm{S}$, et al. Genome-wide association study identifies a sequence variant within the DAB2IP gene conferring susceptibility to abdominal aortic aneurysm. Nat Genet. 2010;42:692-697.

7. Bown MJ, et al. CARDIoGRAM Consortium; Global BPgen Consortium; DIAGRAM Consortium; VRCNZ Consortium. Abdominal aortic aneurysm is associated with a variant in low-density lipoprotein receptorrelated protein 1. Am J Hum Genet. 2011;89:619-627.

8. Bradley DT, et al. A variant in LDLR is associated with abdominal aortic aneurysm. Circ Cardiovasc Genet. 2013;6:498-504.

9. Jones GT, et al. A sequence variant associated with sortilin-1 (SORT1) on 1p13.3 is independently associated with abdominal aortic aneurysm. Hum Mol Genet. 2013;22:2941-2947.

10. Harrison SC, et al. Interleukin-6 receptor pathways in abdominal aortic aneurysm. Eur Heart J. 2013;34:37073716.

11. Helgadottir A, et al. The same sequence variant on $9 \mathrm{p} 21$ associates with myocardial infarction, abdominal aortic aneurysm and intracranial aneurysm. Nat Genet. 2008;40:217-224.

12. Jones GT, et al. Meta-Analysis of Genome-Wide Association Studies for Abdominal Aortic Aneurysm Identifies Four New Disease-specific Risk Loci. Circ Res 2017;120:341-353.

13. Euesden $\mathrm{J}_{2}$ et al. PRSice: Polygenic Risk Score software. Bioinformatics._2015;31:1466-8.

14. Torkamani A, et al. The personal and clinical utility of polygenic risk scores. Nature Reviews Genetics 2018;19: 581-590.

15. Chatterjee N, et al. Developing and evaluating polygenic risk prediction models for stratified disease prevention. Nat. Rev. Genet. 2016;17,392-406.

16. International Schizophrenia Consortium. Common polygenic variation contributes to risk of schizophrenia that overlaps with bipolar disorder. Nature. 2009;460(7256): 748-752.

17. Hurks R, et al. Aneurysm-Express: human abdominal aortic aneurysm wall expression in relation to heterogeneity and vascular events - rationale and design. Eur Surg Res. 2010:45:34-40.

18. Laurie CC, et al. Quality control and quality assurance in genotypic data for genome-wide association studies. Genetic Epidemiology 2010; 34:591-602.

19. The 1000 Genomes Project Consortium. A global reference for human genetic variation. Nature 2015;526,10;68-74.

20. Goldstein JL, et al. zCall: a rare variant caller for array-based genotyping. Bioinformatics 2012; 28,19;25432545.

21. Deelen P, et al. Improved imputation quality of low-frequency and rare variants in European samples using the 'Genome of The Netherlands'. European Journal of Human Genetics 2014;22, 1321-1326.

22. Howie B, et al. Fast and accurate genotype imputation in genome-wide association studies through prephasing. Nature Genetics. 2012;44:955-959.

23. Delaneau O, et al. A linear complexity phasing method for thousands of genomes. Nature methods. 2012;9:179181.

24. Demontis D, et al. Discovery of the first genome-wide significant risk loci for attention deficit/hyperactivity disorder. Nature Genetics 2019;51:63-75.

25. Purcell S, et al. PLINK: A Tool Set for Whole-Genome Association and Population-Based Linkage Analyses. The American Journal of Human Genetics 2007; 81;9;559-575. 
26. Marchini J, et al. A new multipoint method for genome-wide association studies by imputation of genotypes. Nature Genetics. 2007;39:906-913.

27. Pulit SL, et al. Associations Claims in the Sequencing Era. Genes 2014, 5, 196-213; doi:10.3390/genes5010196

28. Aday AW, et al. Vascular genetics: presentations, testing and prognostics. Curr Treat Options Cardio Med 2018; 20:103.

29. Wanhainen A, et al. European Society for Vascular Surgery (ESVS) 2019 Clinical Practice Guidelines on the Management of Abdominal Aorto-iliac Artery Aneurysms, European Journal of Vascular and Endovascular Surgery 2018.

30. van $t$ Hof FNG, et al. Genetic risk load according to the site of intracranial aneurysms. Neurology 2014;83:3439.

31. van $t$ Hof FNG, et al. Impact of Inherited Genetic Variants Associated With Lipid Profile, Hypertension, and Coronary Artery Disease on the Risk of Intracranial and Abdominal Aortic Aneurysms. Circ Cardiovasc Genet. 2013;6:264-270.

32. van $\mathrm{t}$ Hof FN et al. Shared Genetic Risk Factors of Intracranial, Abdominal, and Thoracic Aneurysms. J Am Heart Assoc. 2016 Jul 14;5.

33. Fiotti N, et al. Multiple sites of vascular dilation or aneurysmal disease and matrix metalloproteinase genetic variants in patients with abdominal aortic aneurysm. J Vasc Surg 2017:1-9.

34. Adovasio R, et al. Growth Rate of Small Abdominal Aortic Aneurysms and Genetic Polymorphisms of Matrix MetalloProteases-1, -3, and -9. Int J Angiol 2016;25:93-98.

35. Makrygiannis G, et al. Risk Factor Assessment in a Greek Cohort of Patients With Large Abdominal Aortic Aneurysms. Angiology 2019; 1: 35-40.

36. Ye Z, et al. A multi-locus genetic risk score for abdominal aortic aneurysm. Atherosclerosis. 2016; 246: 274-279.

37. Natarajan P, et al. Polygenic risk score identifies subgroup with higher burden of atherosclerosis and greater relative benefit from statin therapy in the primary prevention setting. Circulation 2017; 135, 2091-2101.

38. Paquette $\mathrm{M}$, et al. Polygenic risk score predicts prevalence of cardiovascular disease in patients with familial hypercholesterolemia. J. Clin. Lipidol (2017) 11, 725-732.e5.

39. Maas $\mathrm{P}$, et al. Breast cancer risk from modifiable and nonmodifiable risk factors among white women in the United States. JAMA Oncol. 2016; 2, 1295-1302. 


\section{Supplemental Material}

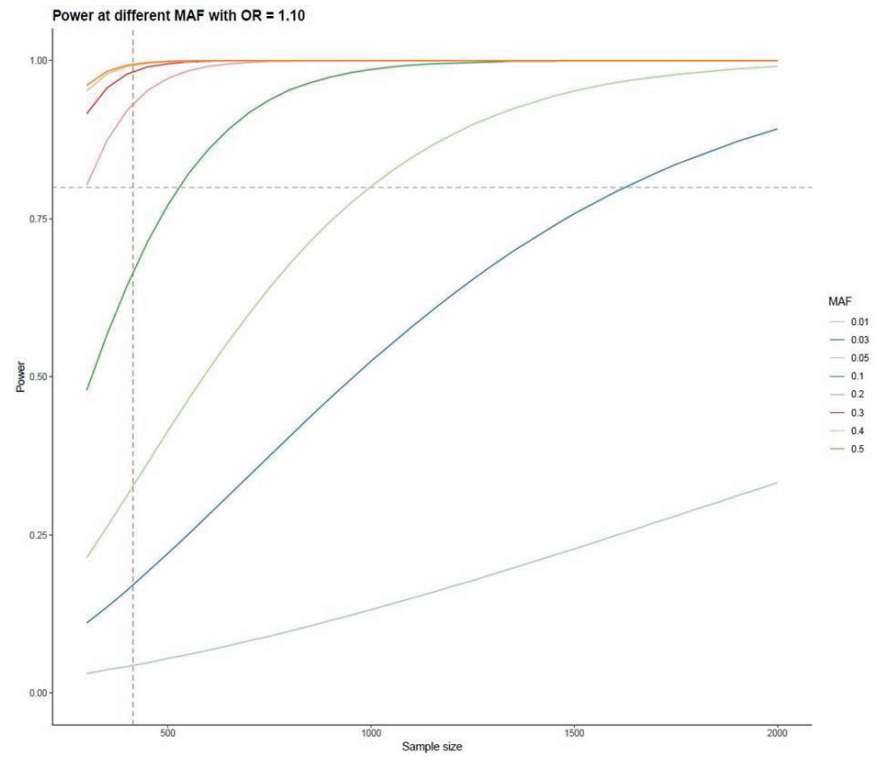

Supplemental Figure I. Plot indicating study power estimates at different minor allele frequencies (MAF), with an average odds ratio (OR) of 1.10. Each solid colored line indicates a different MAF. Red dashed line indicates present study size of 415 samples, grey dashed line indicates a power estimate of $\pm 80 \%$ for risk variants $\mathrm{MAF}<0.2$.

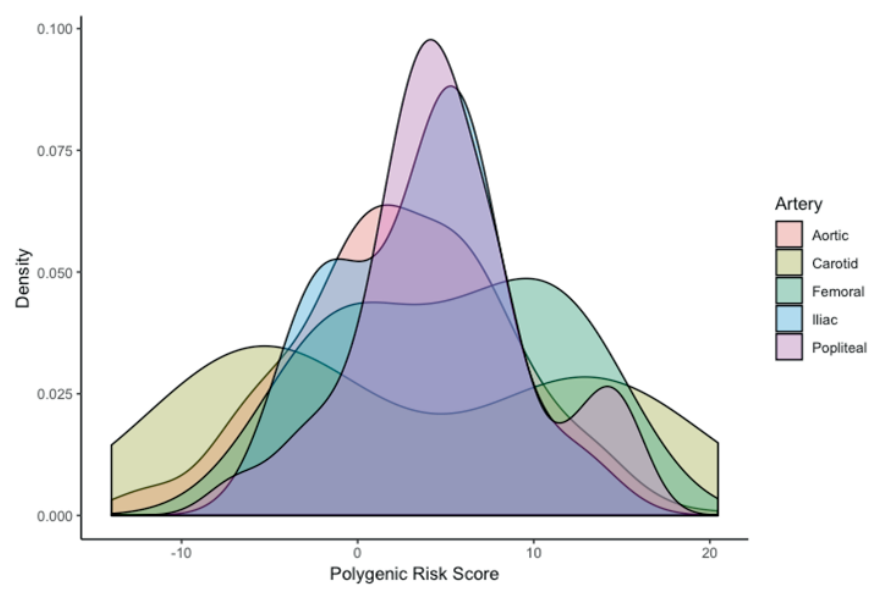

Supplemental Figure II. Overlay density plot of polygenic risk score (PRS) for diameter per artery type. Distribution of PRS is comparable per artery type (Kruskal-Wallis test $p$-value $=0.135)$. 
Supplemental Table I. Polygenic models.

A baseline model was constructed correcting for age, sex, ancestral background using four principal components, smoking status, and maximum diameter of the aneurysm sac or artery type if applicable. $p_{T:}$ the GWAS p-value threshold of association.

\begin{tabular}{ll}
\hline Model & Includes \\
\hline GWAS & Baseline + GWAS hits $p_{T}<5 \times 10^{-8}$ \\
\hline$p<5 \times 10^{-6}$ & Baseline + all SNPs $p_{T}<5 \times 10^{-6}$ \\
\hline$p<5 \times 10^{-4}$ & Baseline + all SNPs $p_{T}<5 \times 10^{-4}$ \\
\hline$p<0.001$ & Baseline + all SNPs $p_{T}<0.001$ \\
\hline$p<0.01$ & Baseline + all SNPs $p_{T}<0.01$ \\
\hline$p<0.05$ & Baseline + all SNPs $p_{T}<0.05$ \\
\hline$p<0.1$ & Baseline + all SNPs $p_{T}<0.1$ \\
\hline$p<0.2$ & Baseline + all SNPs $p_{T}<0.2$ \\
\hline$p<0.5$ & Baseline + all SNPs $p_{T}<0.5$ \\
\hline
\end{tabular}


Supplemental Table II. Detailed polygenic risk score (PRS) models derived from AAA GWAS ${ }^{12}$ for selected clinical phenotypes.

\begin{tabular}{cccccc} 
Diameter of the aneurysm sac & & & & \\
\hline $\boldsymbol{p}_{T}$ & $\mathbf{R}^{2}$ & $\boldsymbol{\beta}$ & $\mathbf{S E}$ & $\boldsymbol{p}$-value & Number of SNPs \\
\hline $5 \times 10^{-8}$ & 0.001 & -1.932 & 2.143 & 0.368 & 1 \\
\hline $5 \times 10^{-6}$ & 0.000 & 0.075 & 1.419 & 0.958 & 3 \\
\hline $5 \times 10^{-4}$ & 0.001 & 0.371 & 0.431 & 0.390 & 24 \\
\hline 0.001 & 0.004 & 0.493 & 0.299 & 0.100 & 40 \\
\hline 0.01 & 0.018 & 0.394 & 0.116 & $0.001^{*}$ & 267 \\
\hline $\mathbf{0 . 0 1 0 1 5}$ & $\mathbf{0 . 0 1 9}$ & $\mathbf{0 . 3 9 8}$ & $\mathbf{0 . 1 1 5}$ & $\mathbf{0 . 0 0 1 ^ { * }}$ & $\mathbf{2 7 2}$ \\
\hline 0.05 & 0.004 & 0.095 & 0.061 & 0.120 & 1,008 \\
\hline 0.1 & 0.004 & 0.075 & 0.046 & 0.105 & 1,634 \\
\hline 0.2 & 0.004 & 0.054 & 0.036 & 0.133 & 2,494 \\
\hline 0.5 & 0.004 & 0.045 & 0.029 & 0.125 & 4,086 \\
\hline
\end{tabular}

\section{Artery type}

\begin{tabular}{cccccc}
\hline $\boldsymbol{p}_{\boldsymbol{T}}$ & $\mathbf{R}^{2}$ & $\boldsymbol{\beta}$ & $\mathbf{S E}$ & $\boldsymbol{p}$-value & Number of SNPs \\
\hline $5 \times 10^{-8}$ & 0.005 & -0.886 & 0.681 & 0.193 & 1 \\
\hline $5 \times 10^{-6}$ & 0.003 & -0.382 & 0.411 & 0.353 & 3 \\
\hline $5 \times 10^{-4}$ & 0.000 & 0.055 & 0.138 & 0.688 & 24 \\
\hline 0.001 & 0.004 & 0.110 & 0.096 & 0.252 & 39 \\
\hline $\mathbf{0 . 0 0 2 2}$ & $\mathbf{0 . 0 0 7}$ & $\mathbf{0 . 0 9 9}$ & $\mathbf{0 . 0 6 3}$ & $\mathbf{0 . 1 1 4}$ & $\mathbf{8 8}$ \\
\hline 0.01 & 0.007 & 0.056 & 0.038 & 0.140 & 267 \\
\hline 0.05 & 0.030 & 0.020 & 0.020 & 0.321 & 1,008 \\
\hline 0.1 & 0.001 & 0.006 & 0.014 & 0.652 & 1,632 \\
\hline 0.2 & 0.000 & 0.000 & 0.012 & 0.989 & 2,493 \\
\hline 0.5 & 0.000 & -0.001 & 0.009 & 0.950 & 4,086 \\
\hline
\end{tabular}

\section{Symptom status}

\begin{tabular}{cccccc}
\hline $\boldsymbol{p}_{T}$ & $\mathbf{R}^{2}$ & $\boldsymbol{\beta}$ & $\mathbf{S E}$ & $\boldsymbol{p}$-value & Number of SNPs \\
\hline $5 \times 10^{-8}$ & 0.000 & 0.032 & 0.342 & 0.925 & 1 \\
\hline $5 \times 10^{-6}$ & 0.000 & 0.023 & 0.226 & 0.921 & 24 \\
\hline $5 \times 10^{-4}$ & 0.003 & 0.064 & 0.069 & 0.350 & 39 \\
\hline 0.001 & 0.001 & 0.025 & 0.049 & 0.604 & 270 \\
\hline 0.01 & 0.002 & 0.013 & 0.019 & 0.491 & 1,009 \\
\hline 0.05 & 0.002 & 0.007 & 0.010 & 0.483 & $\mathbf{0 . 1 7 3}$ \\
\hline $\mathbf{0 . 0 6 3 7}$ & $\mathbf{0 . 0 0 7}$ & $\mathbf{0 . 0 1 2}$ & $\mathbf{0 . 0 0 9}$ & 0.768 & 1,632 \\
\hline 0.1 & 0.000 & 0.002 & 0.007 & 0.692 & 2,494 \\
\hline 0.2 & 0.001 & 0.002 & 0.006 & 0.818 & 4,086 \\
\hline 0.5 & 0.000 & 0.001 & 0.005 & & \\
\hline
\end{tabular}

Bold indicates best fitted model, ${ }^{*}$ indicates $p<0.05$.

Abbrevations: $p_{T}=p$-value threshold. $\beta=$ beta coefficient. $\mathrm{SE}=$ standard error, SNPs $=$ single nucleotide polymorphisms. 
Supplemental Table III. Detailed polygenic risk score (PRS) models derived from attention deficit hyperactivity disorder GWAS $^{24}$ for selected clinical phenotypes.

\begin{tabular}{cccccc}
\hline Diameter of the aneurysm sac & \multicolumn{1}{c}{} & \\
\hline $\boldsymbol{p}_{T}$ & $\mathbf{R}^{2}$ & $\boldsymbol{\beta}$ & $\mathbf{S E}$ & $\boldsymbol{p}$-value & Number of SNPs \\
\hline $5 \times 10^{-8}$ & 0.003 & -0.973 & 0.714 & 0.174 & 10 \\
\hline $\mathbf{5 \times 1 0 ^ { - 6 }}$ & $\mathbf{0 . 0 0 4}$ & $\mathbf{- 1 . 1 0 5}$ & $\mathbf{0 . 7 0 3}$ & $\mathbf{0 . 1 1 7}$ & $\mathbf{7 7}$ \\
\hline $5 \times 10^{-4}$ & 0.000 & -0.256 & 0.710 & 0.719 & 1,179 \\
\hline 0.001 & 0.000 & 0.306 & 0.716 & 0.670 & 1,858 \\
\hline 0.01 & 0.002 & -0.702 & 0.727 & 0.335 & 8,155 \\
\hline 0.05 & 0.002 & -0.907 & 0.745 & 0.224 & 20,395 \\
\hline 0.1 & 0.001 & -0.494 & 0.745 & 0.507 & 28,657 \\
\hline 0.2 & 0.000 & -0.298 & 0.741 & 0.688 & 39,167 \\
\hline 0.5 & 0.000 & -0.409 & 0.741 & 0.581 & 55,850 \\
\hline
\end{tabular}

\section{Artery type}

\begin{tabular}{cccccc}
\hline $\boldsymbol{p}_{T}$ & $\mathbf{R}^{2}$ & $\boldsymbol{\beta}$ & $\mathbf{S E}$ & $\boldsymbol{p}$-value & Number of SNPs \\
\hline $5 \times 10^{-8}$ & 0.000 & 0.086 & 0.230 & 0.707 & 10 \\
\hline $\mathbf{5 \times 1 0 ^ { - 6 }}$ & $\mathbf{0 . 0 1 0}$ & $\mathbf{- 0 . 4 2 4}$ & $\mathbf{0 . 2 3 4}$ & $\mathbf{0 . 0 7 0}$ & $\mathbf{7 7}$ \\
\hline $5 \times 10^{-4}$ & 0.001 & -0.168 & 0.240 & 0.486 & 1,179 \\
\hline 0.001 & 0.000 & -0.045 & 0.239 & 0.851 & 1,858 \\
\hline 0.01 & 0.001 & -0.115 & 0.235 & 0.625 & 8,155 \\
\hline 0.05 & 0.003 & -0.254 & 0.239 & 0.289 & 20,395 \\
\hline 0.1 & 0.000 & -0.006 & 0.226 & 0.979 & 28,657 \\
\hline 0.2 & 0.000 & -0.008 & 0.232 & 0.972 & 39,167 \\
\hline 0.5 & 0.000 & -0.026 & 0.234 & 0.913 & 55,850 \\
\hline
\end{tabular}

\section{Symptom status}

\begin{tabular}{cccccc}
\hline $\boldsymbol{p}_{T}$ & $\mathbf{R}^{2}$ & $\boldsymbol{\beta}$ & $\mathbf{S E}$ & $\boldsymbol{p}$-value & Number of SNPs \\
\hline $5 \times 10^{-8}$ & 0.000 & 0.028 & 0.115 & 0.809 & 10 \\
$\mathbf{5 \times 1 0 ^ { - 6 }}$ & $\mathbf{0 . 0 1 0}$ & $\mathbf{- 0 . 1 8 4}$ & $\mathbf{0 . 1 1 4}$ & $\mathbf{0 . 1 0 6}$ & $\mathbf{7 7}$ \\
\hline $5 \times 10^{-4}$ & 0.001 & -0.070 & 0.113 & 0.533 & 1,179 \\
\hline 0.001 & 0.002 & -0.081 & 0.114 & 0.481 & 1,858 \\
\hline 0.01 & 0.000 & -0.038 & 0.116 & 0.740 & 8,155 \\
\hline 0.05 & 0.000 & -0.032 & 0.119 & 0.791 & 20,395 \\
\hline 0.1 & 0.001 & -0.071 & 0.120 & 0.553 & 28,657 \\
\hline 0.2 & 0.001 & -0.074 & 0.119 & 0.531 & 39,167 \\
\hline 0.5 & 0.000 & -0.005 & 0.118 & 0.969 & 55,850 \\
\hline
\end{tabular}

Bold indicates best fitted model, ${ }^{*}$ indicates $p<0.05$.

Abbrevations: $p_{T}=p$-value threshold. $\beta=$ beta coefficient. $\mathrm{SE}=$ standard error, SNPs $=$ single nucleotide polymorphisms. 
Supplemental Table IV. Clinical characteristics of the upper $20^{\text {th }}$ percentile and lower $80^{\text {th }}$ percentile of PRS.

\begin{tabular}{|c|c|c|c|c|c|}
\hline \multirow[b]{2}{*}{ Male sex } & \multicolumn{2}{|c|}{$\begin{array}{c}\text { Lower } 80^{\text {th }} \text { of PRS } \\
n=332\end{array}$} & \multicolumn{2}{|c|}{$\begin{array}{c}\text { Upper } 20^{\text {th }} \text { of PRS } \\
n=83\end{array}$} & \multirow{2}{*}{$\begin{array}{c}p \\
0.371^{a}\end{array}$} \\
\hline & 285 & $(81 \%)$ & 68 & $(19 \%)$ & \\
\hline Age at surgery, median (range) & 69.5 & $(46-89)$ & 71.0 & $(28-87)$ & $0.460^{\mathrm{b}}$ \\
\hline In-hospital days, median (range) & 9 & $(1-81)$ & 10 & $(1-65)$ & $0.216^{\mathrm{b}}$ \\
\hline Any in-hospital reintervention post-operatively & 37 & $(13 \%)$ & 9 & $(12 \%)$ & $0.747^{\mathrm{a}}$ \\
\hline In-hospital death & 16 & $(6 \%)$ & 3 & $(4 \%)$ & $0.576^{\mathrm{a}}$ \\
\hline \multicolumn{6}{|l|}{ Aneurysm } \\
\hline Aortic & 281 & $(81 \%)$ & 69 & $(19 \%)$ & $0.311^{\mathrm{a}}$ \\
\hline Iliac & 8 & $(62 \%)$ & 5 & $(39 \%)$ & \\
\hline Femoral & 7 & $(87 \%)$ & 1 & $(13 \%)$ & \\
\hline Popliteal & 28 & $(80 \%)$ & 7 & $(20 \%)$ & \\
\hline Carotid & 8 & $(89 \%)$ & 1 & $(11 \%)$ & \\
\hline IL- $6^{*}$, mean \pm sd & 4.0 & \pm 1.8 & 3.7 & \pm 2.3 & $0.321^{\mathrm{c}}$ \\
\hline IL-8 $^{*}$ & 4.6 & \pm 1.5 & 4.1 & \pm 1.6 & $0.051^{c}$ \\
\hline MCP-1* & 1.9 & \pm 2.1 & 1.8 & \pm 2.0 & $0.782^{c}$ \\
\hline OPG $^{*}$ & 8.3 & \pm 1.0 & 8.3 & \pm 1.0 & $0.964^{c}$ \\
\hline
\end{tabular}

Abbreviations: $\mathrm{PRS}=$ polygenic risk score, $\mathrm{IL}=$ interleukin, $\mathrm{sd}=$ standard deviation, $\mathrm{MCP}=$ monocyte chemotactic protein, $\mathrm{OPG}=$ osteoprotegerin. ${ }^{\star}$ natural $\log$ transformed, all inflammatory markers are extracted from the tissue sample, analyzed by Luminex and corrected for inter assay variability with pooled and blank sample.

${ }^{a}$ Chi-squared test, ${ }^{b}$ Mann-Whitney U test, ${ }^{c}$ Student's T-test. 


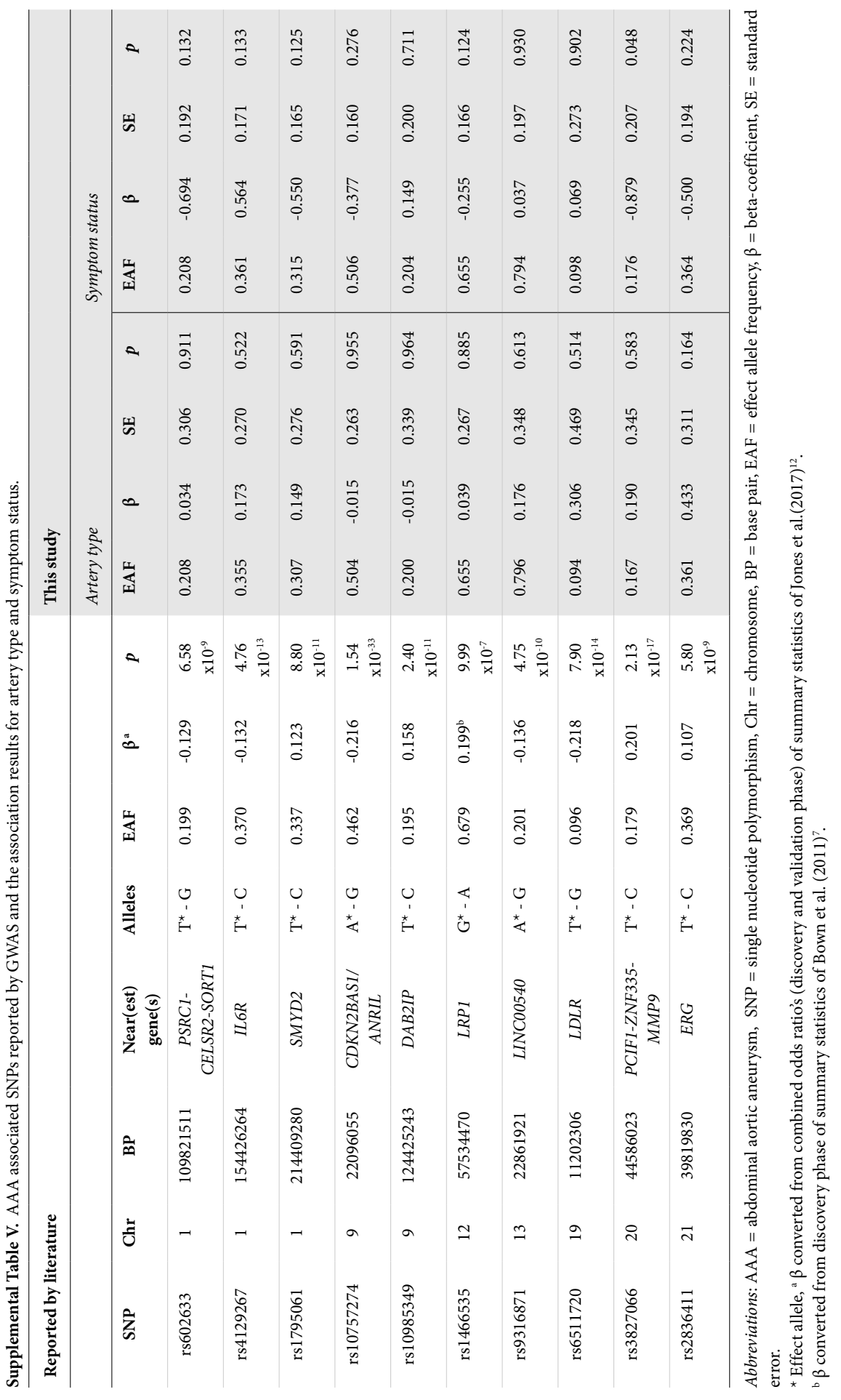




\section{Part II}
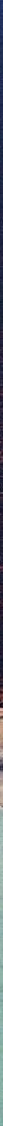



\section{Chapter 7}

\section{Volumetric assessment of extracranial carotid artery aneurysms}

Sci Rep 2019;30:8108

C.J.H.C.M. van Laarhoven*

E.E. de Vries*

H.J. Kuijf

C.E.V.B. Hazenberg

J.A. van Herwaarden

M.A. Viergever

G.J. de Borst

* Shared first authorship 


\section{Abstract}

\section{Background and purpose}

The extracranial carotid artery aneurysm (ECAA) is a rare pathology for which clinical treatment guidelines are lacking. In general, symptoms or growth of the aneurysm sac are thought to indicate intervention. ECAAs may present in a large variety of shapes and sizes, and conventional diameter measurements fail to indicate geometrical differences. Therefore, we propose a protocol to measure ECAA size by $3 \mathrm{D}$ volumetric assessment.

\section{Methods}

The volumes of 40 ECAAs in computed tomography angiography (CTA) images were measured through manual segmentation, by two independent operators. Volumes of the entire internal carotid artery (ICA) and the ECAA were measured separately.

\section{Results}

Excellent inter- and intraoperator reliability was found for both ICA and ECAA volumes, with all intraclass correlation coefficients above 0.94 . Bland-Altman analysis revealed normal differences for both inter- and intraoperator agreement. For all volumes, similarity of the segmentations was excellent. Outliers were explained by presence of intraluminal ECAA thrombus, which hampered identification of the aneurysm outer wall.

\section{Conclusion}

These results implicate robustness of our protocol, which is designed as a step-up towards (semi)automatic volumetric measurements to monitor patients with ECAA. Future (semi) automatic volumetric assessments are recommended and such techniques can be developed and validated using the proposed protocol and manual reference segmentations. 


\section{Introduction}

The extracranial carotid artery aneurysm (ECAA) is a rare vascular pathology, of which the natural clinical course, and risk factors for adverse outcome are largely unknown ${ }^{1,2}$. As a consequence, currently no evidence-based guidelines exist on treatment of ECAAs. Asymptomatic, non-growing aneurysms are generally treated conservatively ${ }^{3}$, while surgical or endovascular aneurysm exclusion may be considered in case of symptomatic or growing ECAA ${ }^{2}$. However, a standardized method to measure ECAA size or growth is currently not available. At present, ECAA size is mostly defined by determination of maximum diameter. These diameters can be assessed by measuring the bi-directional maximum aneurysm diameter from outer to outer vessel wall, extracted from the wellestablished approach for abdominal aortic aneurysms (AAAs) ${ }^{4-6}$. However, ECAAs may present in a large variety of shapes and sizes. The dilatation of ECAA may be focal and saccular, or extensive and fusiform, or a combination of both, and maximum aneurysm diameters between 4 and $60 \mathrm{~mm}$ have been reported ${ }^{3,7,8}$. Furthermore, it is imaginable that ECAA growth might occur in other directions than just obliquely. Thus, assessment of geometrical differences by comparing maximum aneurysm diameters is inadequate as we lack the appropriate imaging tools. A standardized protocol to measure 3D ECAA volume - instead of 2D diameters - would theoretically be able to reliably determine aneurysm size and growth. These measures could then be used for follow-up of ECAA patients (and ultimately for patients with any type of aneurysm), for pre-treatment monitoring, and underpinning of treatment decisions. Therefore, the aim of this study was to develop and evaluate a robust protocol for volume measurement of ECAAs.

\section{Methods}

\section{Participants}

Twenty patients with an ECAA located in the internal carotid artery (ICA) gave informed consent and were included from our Carotid Aneurysm Registry (www. carotidaneurysmregistry.com). Ethical approval for this study (12/020761) was provided by the Medical Research Ethics Committee of University Medical Center Utrecht, Utrecht, The Netherlands on 12 June 2012, and all research was conducted according to the principles of the Declaration of Helsinki (59th amendment, Seoul 2008) and in accordance with the Dutch Medical Research Involving Human Subjects Act (WMO). The registry protocol has been published elsewhere ${ }^{9}$. Briefly, any patient aged 18-years or older with an ECAA is included in this ongoing registry, independent of etiology or treatment strategy. Patients who, or their legal representative, are unable or unwilling to sign the informed consent were excluded. Selected patients for the current study had two computed tomography angiography (CTA) scans of the carotids made at different time 
points. Hence, in total 40 scans were included. The CTAs had been performed for evaluation or treatment planning of ECAAs between 2008 and 2017, in the University Medical Center Utrecht. As specified within the registry protocol ${ }^{9}$, fusiform aneurysms were defined as $\geq 150 \%$ diameter increase of the non-affected ICA diameter, while saccular aneurysms were defined as a distended sac of any size affecting only part of the ICA circumference.

\section{Volume measurement protocol}

The protocol for volume measurement of ECAAs was developed by an expert panel, which consisted of three vascular surgeons (G.B., J.H., C.H.) and two dedicated researchers (E.V., C.L.). Each case was measured in a twofold manner. First, the volume of the entire ICA (including ECAA) was measured, from carotid bifurcation up until the entrance of the ICA in the skull base (method 1). Hereafter, the ECAA volume was measured separately (method 2 ).

\section{Method 1: Entire ICA}

The starting point of the ICA was the carotid bifurcation (Figure 1). On sagittal and coronal views, the ipsilateral common and external carotid arteries were segmented, and subtracted from the segmented proximal ICA on axial view. Distally, the ICA was segmented up until entrance in the skull base, defined as the first slice which showed at least $50 \%$ of the ICA surrounded by bone.

\section{Method 2: Solely the ECAA}

Since ECAAs come in different shapes, a different approach was inevitable to define the borders of saccular versus fusiform aneurysms.

\section{A: Fusiform aneurysms}

The aneurysm was segmented from the clear starting point of vessel dilatation onwards (immediately $\geq 150 \%$; Figure $2 \mathrm{~A}$ ). If the widening of the vessel commenced gradually, segmentation started when the vessel had widened with $\geq 125 \%$ compared with the healthy ipsilateral ICA.

\section{B: Saccular aneurysms}

All saccular ECAAs were measured similarly. First, volume of just the aneurysm sac was measured. In case the outer wall of the aneurysm sac adjoined the outer wall of the ICA, volume of the aneurysm sac was additionally measured in combination with the part of the ICA adjacent to the aneurysm neck and sac (Figure 2B). Hence, both separate segmentations were added to calculate volume of Method 2 (Figure 3).

For a schematic overview of the twenty cases, we refer to Appendix A. The final segmentations as performed with MeVisLab (see next subsection) are included in Appendix B. A case example with color coding and names of separate volumes is shown in Figure 3. 


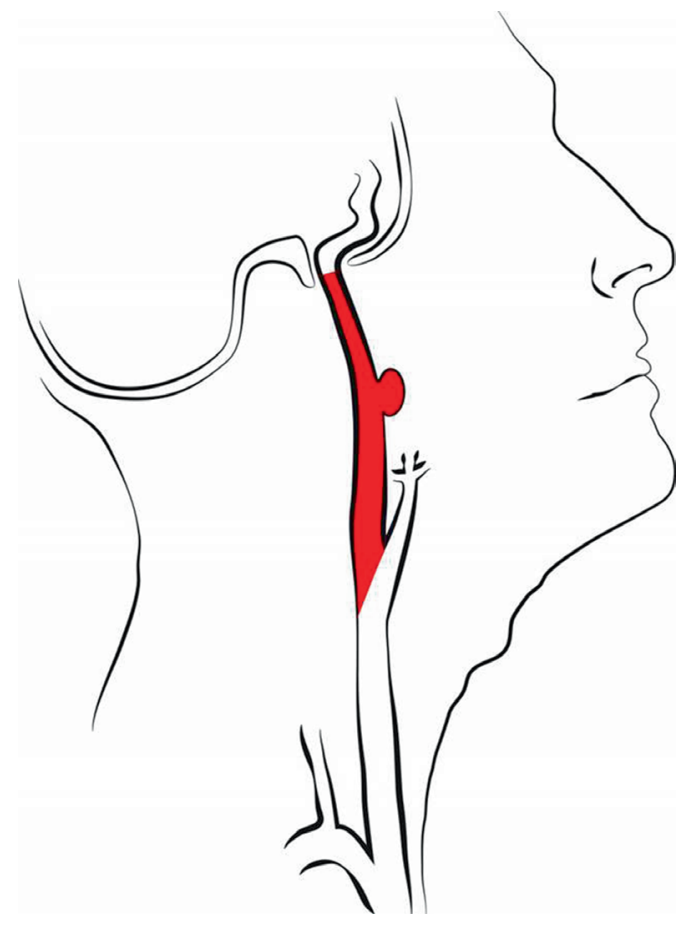

Figure 1. Measurement method 1: Entire ICA.

\section{Software}

In-house developed software (by H.K.) implemented in MeVisLab (MeVis Medical Solutions AG, Bremen, Germany) was used to manually trace the ICA and borders of the ECAA. This program provided a user-friendly spline drawing and editing technique, with which we were able to follow the borders of the structures closely. All volumes were manually segmented from outer to outer wall, as is standard in segmentation of abdominal aortic aneurysms ${ }^{4-6}$. All cases were segmented on axial view (sagittal and coronal views were only used to identify structure borders). Volumes (in $\mathrm{mL}$ ) were obtained by multiplying the size of the segmented volume by the voxel size of the acquired image.

\section{Study design}

Two operators (E.V., C.L.) independently segmented each of the 40 scans according to the measurement methods 1 and 2 in the predefined protocol. Prior to measurement of the 40 scans, both operators measured three test scans in order to familiarize with the program. The results of these test segmentations were compared to overcome potential differences in protocol interpretation. Afterwards, the twenty cases were offered to the operators in randomized fashion in order to prevent a potential effect from learning. 

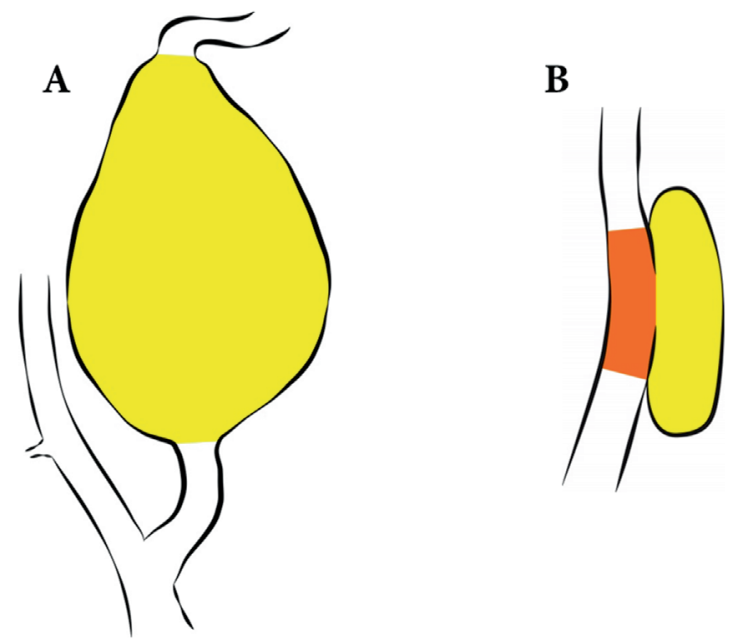

Figure 2. Measurement method 2: Solely the ECAA. (A) Fusiform aneurysm. (B) Saccular aneurysm: measurement of solely the aneurysm sac (yellow), and vessel adjacent to aneurysm sac (orange).

Of each case, the first and second scans were measured in succession. A random sample of five ECAAs was selected for both operators to be measured twice according to method 2 .

\section{Imaging}

A 64-slice or 128-slice CT scanner (Philips Brilliance; Philips medical systems, Best, The Netherlands) was used to acquire the CTA scans. The carotid arteries were visualized from the aortic arch up until the skull base. The median slice thickness was $0.9 \mathrm{~mm}$ (range $0.6-3.0 \mathrm{~mm}$ ), increment $0.33 \mathrm{~mm}$, pitch $0.609 \mathrm{~mm}$, and collimation $64 \mathrm{x} 0.625 \mathrm{~mm}$. Tube voltage was $100-120 \mathrm{kV}$ and tube current 17-300 mA. Fields of view were set per patient. Injection of 65 milliliter intravascular contrast (ultravist 300, Schering, Berlin, Germany) was followed by a saline bolus of 40 milliliter, both at a flow rate of 6 milliliters per second.

\section{Outcomes and statistical analysis}

Crude volumes were calculated for method 1 (entire internal carotid artery ipsilateral to the ECAA) and method 2 (solely the ECAA).

The primary outcomes were measurement reliability and agreement of the protocol. Interoperator reliability of each volume was calculated by the intraclass correlation coefficient (ICC; model: two-way mixed, type: consistency). This was done for the entire ICA (method 1), as well as for the ECAA (method 2, A and B). An ICC of 1.0 equals perfect reliability, an ICC of 0.81-0.99 excellent reliability, and an ICC of 0.61-0.80 substantial reliability ${ }^{10}$. Intraoperator reliability for measurement method 2 was calculated 


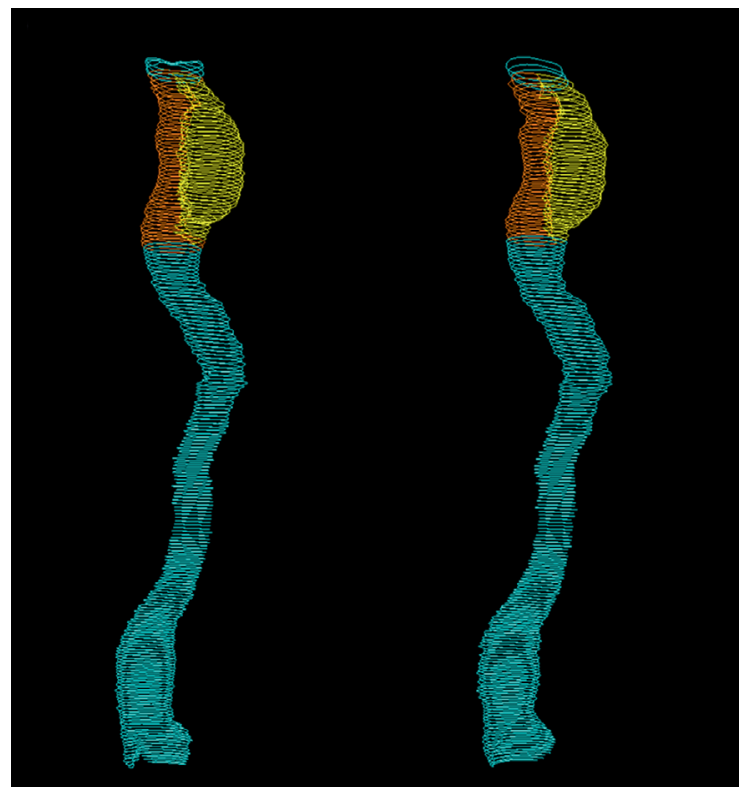

Figure 3. Example of segmentation of operator 1 (left) and operator 2 (right). The separate segmentations are referred to "only vessel" in blue, "aneurysm sac" in yellow, and "vessel adjacent to aneurysm sac" in orange. Total volume of method 1 (entire ICA) is calculated by adding up the volumes of all three separate segmentations. Total volume of method 2 (the ECAA) is calculated by adding up the volumes of "aneurysm sac" and "vessel adjacent to aneurysm sac".

for both operators for the random sample of five scans. Inter- and intraoperator agreement was assessed by use of Bland-Altman analysis.

In addition, Dice Similarity Coefficients (DSCs) and modified Hausdorff Distances (HDs) were calculated for measurement methods 1 and 2, to assess similarity of the segmentations. The DSC is a measure of spatial overlap of two segmentations, with perfectly overlapping segmentations resulting in a Dice score of 1 , and a complete lack of overlap resulting in a Dice score of $0^{11}$. The HD measures the boundary distance in $\mathrm{mm}$ between segmentation contours, with a $\mathrm{HD}$ of $0 \mathrm{~mm}$ meaning that the two segmentations are identical ${ }^{12}$. We used a modified $\mathrm{HD}$, using the $95^{\text {th }}$ percentile of the distances over the full segmentation to be less sensitive to outliers ${ }^{13}$. The amount of disagreement that was considered acceptable between segmentations was judged per case, and was mainly dependent on the size of the ECAA and the spatial resolution of the images. All data analyses were conducted using SPSS version 25 (IBM Corp. Released 2017. IBM SPSS Statistics for Windows, Version 25.0. Armonk, NY: IBM Corp.). 


\section{Results}

The median age of the subjects was 51 years (range 36-80 years) at the time of the first CTA scan. $50 \%$ of subjects was male, and aneurysms were fusiform in $35 \%$ of cases. The median time between the first and second scan was 56 months (range 3-184 months). The median aneurysm diameter reported in the patient records at the time of the first scan was $13 \mathrm{~mm}$ (range $9-26 \mathrm{~mm}$ ) for fusiform aneurysms, and $17 \mathrm{~mm}$ (range $7-44 \mathrm{~mm}$ ) for saccular aneurysms. The time needed to perform the manual segmentations of 40 scans was 32.3 hours, or 49 minutes per scan (as an average of both observers).

\section{Evaluation of the protocol}

\section{Interoperator reliability and segmentation similarity}

Interoperator reliability scores are summarized in Table $\mathbf{1}$ and indicate excellent consistency for measurement methods 1 and 2, with all ICCs higher than 0.94 . Similarity as measured with the DSC was above 0.88 for method 1 and 2 , except for the segmentation "vessel adjacent to aneurysm sac" with a DSC of 0.79 . The HD ranged from median 0.801 (interquartile range [IQR] 0.430-7.006) mm for method 1 to median 1.229 (IQR 0.5842.863) $\mathrm{mm}$ for segmentation of the "aneurysm sac".

\section{Intraoperator reliability and segmentation similarity}

Intraoperator reliability for measurement method 2 was calculated for both operators for the random sample of five ECAAs, and was close to perfect with an average intraoperator reliability of 0.999 . Similarity of segmentations as measured with the DSC was above 0.93 for both operators, and HD values were below $1 \mathrm{~mm}$ for both operators (data not shown).

\section{Interoperator agreement}

Bland-Altman plots were constructed to assess presence of systematic differences between both operators for method 1 and 2 (Figure 4A and B, Appendix C). All plots reveal approximately normal differences, as $95 \%$ of all differences fell within the limits of agreement. However, proportional bias can be observed in all plots except "only vessel", since the difference in volume increased with the mean volume.

The measurements that fell outside the upper limit of agreement were cases $7 \mathrm{a}$ and $7 \mathrm{~b}$ for measurement method 1 , and cases $3 \mathrm{~b}$ and $7 \mathrm{~b}$ for measurement method 2 (Figure 4 ). These cases contained aneurysm sacs with a significant amount of thrombus, which hampered identification of the aneurysm outer wall. 
Table 1. Reliability and similarity of both operators for measurement of the entire ICA (method 1), the ECAA (method 2), and the three separate segmentations "only vessel", "aneurysm sac", and "vessel adjacent to aneurysm sac" (also see Figure 3). Reliability was expressed as the Intraclass Correlation Coefficient (ICC), similarity as the Dice Similarity Coefficient (DSC) and modified Hausdorff Distance (HD).

\begin{tabular}{lccc}
\hline & $\begin{array}{c}\text { ICC } \\
(\mathbf{9 5 \%} \mathbf{C I})\end{array}$ & $\begin{array}{c}\text { DSC } \\
(\text { median, IQR) }\end{array}$ & $\begin{array}{c}\text { HD, mm } \\
\text { (median, IQR) }\end{array}$ \\
\hline Method 1 & $0.995(0.991-0.998)$ & $0.913(0.895-0.954)$ & $0.801(0.588-1.728)$ \\
\hline Method 2 & $0.996(0.993-0.998)$ & $0.906(0.860-0.957)$ & $1.229(0.663-2.793)$ \\
\hline Separate segmentations & & & \\
\hline - only vessel & $0.960(0.926-0.979)$ & $0.885(0.857-0.899)$ & $0.947(0.631-1.435)$ \\
\hline - aneurysm sac & $0.996(0.993-0.998)$ & $0.903(0.854-0.955)$ & $1.229(0.584-2.863)$ \\
\hline - vessel adjacent to aneurysm sac & $0.941(0.891-0.968)$ & $0.788(0.736-0.818)$ & $1.208(1.033-2.724)$ \\
\hline
\end{tabular}

Footnotes: ${ }^{*}$ See Figure 3 for case example and description of the separate segmentations.

Abbreviations: ICC, intraclass correlation coefficient; DSC, Dice similarity coefficient; HD, modified Hausdorff distance; IQR, interquartile range; $\mathrm{CI}$, confidence interval.

\section{Discussion}

In this study, we developed a new protocol for volumetric assessment of ECAAs, and demonstrated that this protocol can be applied in a reliable manner with excellent ICC, DSC and HD values.

The use of volume measurements in addition to diameter measurements for detection of aneurysm growth has been a topic of interest in multiple vascular territories. Literature on abdominal aortic aneurysms showed that volumetric measurements may be superior to conventional diameter measurements for detecting size changes as a follow-up tool after endovascular repair ${ }^{14,15}$, as well as untreated aneurysm surveillance ${ }^{16}$. In intracranial aneurysms, a moderate correlation of volume and diameter at best was only found in predominantly saccularly shaped aneurysms, and not at all in fusiform aneurysms ${ }^{17}$. However, despite abundant evidence, volume assessment of aneurysms over diameter measurements to assess size is still not standard practice in most institutions. This is largely because volume assessment is time consuming, requires dedicated software and skilled technicians, and carries significant operator variability ${ }^{18}$. Our volume measurement protocol for ECAA was applied by two operators with excellent levels of reliability and agreement, even though the current manual segmentation process was time-consuming. However, it was designed as a step-up towards development of a (semi)automatic volume measurement tool. This future application aims to minimize the time required to perform the volume assessment, and will bypass the need for skilled technicians. It is crucial to develop a robust and feasible tool to monitor the aneurysm sac, by measuring growth and geometrical alterations, and hereby aid in selection of patients who should undergo intervention to prevent future adverse events. 
A

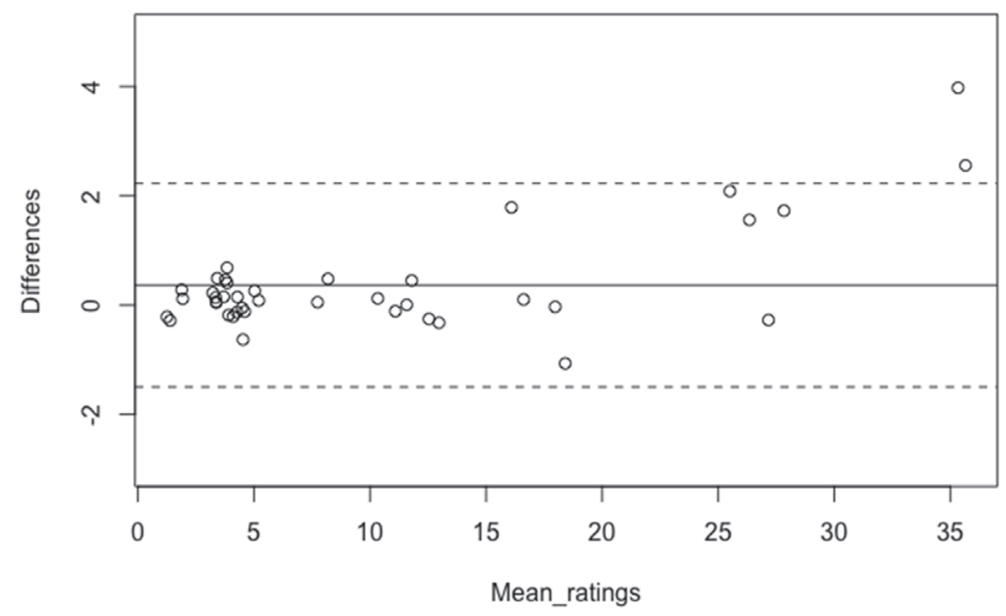

B

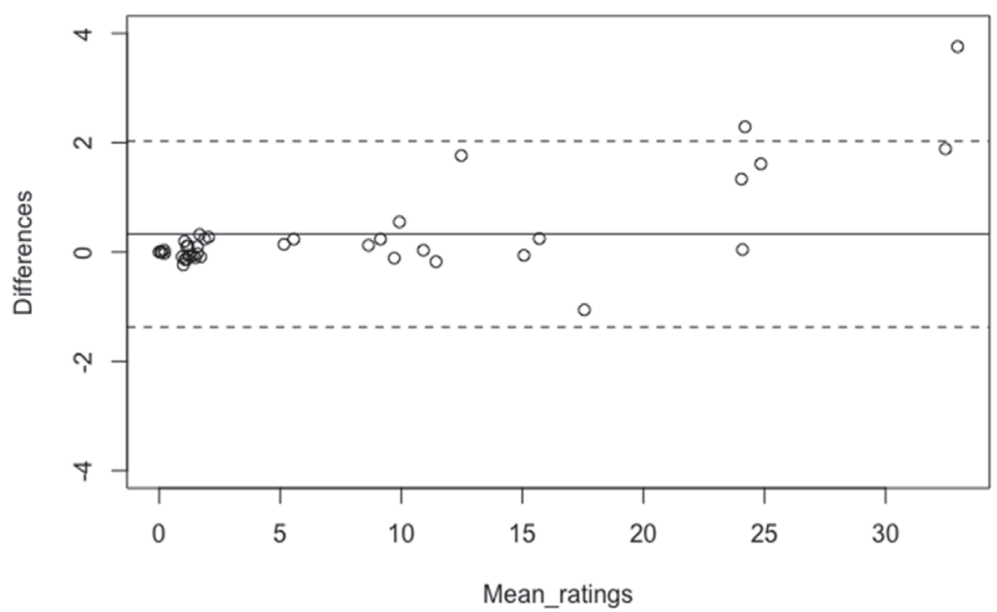

Figure 4. Bland-Altman plots showing agreement of two operators on the volume measurement of 40 scans according to method 1 (A) and method 2 (B). The line in the middle represents the mean difference of the volume (in $\mathrm{mL}$ ) between the two operators, and the dashed lines represent the upper and lower limits of agreement (mean difference $\pm 1.96 \times$ standard deviation). 
Our protocol comprised two measurement methods, segmentation of the entire ICA and solely the ECAA. The specific segmentation of solely the ECAA was demonstrated to be reproducible with excellent interoperator reliability. Segmentation of the entire ICA had been included in the protocol to overcome expected difficulties in identification of aneurysm starting point and terminus. However, even though in case of fusiform aneurysms the cutoff value of $>125 \%$ enlargement on axial slices on CTA was pragmatic and might be considered arbitrary, this did not hamper accurate and similar segmentation between operators.

Median HD values were below $1.3 \mathrm{~mm}$ for all methods of segmentations, which translated into a difference of $2 \mathrm{~mL}$ in volume according to the Bland-Altman plots. The outliers were mostly explained by the two aneurysms which contained a large amount of thrombus alongside the vascular wall. An additional analysis without these two thrombuscontaining aneurysms revealed a median $\mathrm{HD}$ value of $1.1 \mathrm{~mm}$, and a corresponding difference of $1 \mathrm{~mL}$ in volume. This means that the measurement error is $1-2 \mathrm{~mL}$ (depending on in- or exclusion of thrombus-containing aneurysms), and that a difference in ECAA volume should be at least $1-2 \mathrm{~mL}$ to allow detection with our volume measurement protocol. We consider this difference negligible for all aneurysms irrespective of their initial size, and believe this measurement error is small enough for our protocol to allow adequate detection of ECAA growth.

For this project we had to face several challenges. First, although we use a common definition for ECAA shape, this definition remains somewhat arbitrary, and mixed forms exist next to saccular and fusiform shapes. As our protocol required a different measurement approach for saccular and fusiform aneurysms, it was crucial to establish the ECAA morphology first and start the corresponding measurements thereafter. In addition, even though separate segmentations were reproducible with high ICC, DSC, and HD values (Table 1, Figure 3), the distinction between 'aneurysm sac' and 'vessel adjacent to aneurysm sac' might be judged clinically irrelevant. Therefore, both volumes were added up to calculate aneurysm volume (Method 2). Second, although significant thrombus formation within an ECAA is relatively rare, some ECAA do contain thrombus which is likely to challenge segmentation of the aneurysm sac (as can be seen in cases 3 and 7 with relatively low interoperator agreements). Therefore, in this stage, our protocol is only workable for ECAA which contain no or minimal thrombus, and future studies need to focus on better visualization techniques to enable volume measurements of the challenging ECAA with significant thrombus. Third, a thick slice $3.0 \mathrm{~mm}$ CTA scan is likely to be less accurate than a thin slice $0.6 \mathrm{~mm}$ CTA scan for volume measurements, and a discrepancy in slice thickness in two paired scans performed at different time points might introduce bias. Therefore, all CTA scans should have similar thin slice thickness in order to enable accurate conclusions about ECAA growth. Lastly, it is unknown how little changes in head posture during scanning might influence ECAA and ICA volumes. 
We hypothesize that $3 \mathrm{D}$ volume measurements are less sensitive to these postural changes than conventional 2D diameter measurements. Nevertheless, studies are advised to scan patients with their head in locked position to rule out confounding due to different head and neck postures.

\section{Conclusion}

This proposed protocol to measure ECAA volumes revealed excellent inter- and intraoperator reliability and agreement, as well as excellent similarity of the segmentations performed by two independent operators. Future volumetric assessments are recommended to be included in standardized imaging protocols, which should also focus on head and neck posture, and visualization of thrombus and vessel wall. These could be used as a step-up to (semi)automatic volumetric measurements to monitor patients with ECAA. 


\section{References}

1. Welleweerd, J. C. et al. Management of Extracranial Carotid Artery Aneurysm. Eur. J. Vasc. Endovasc. Surg. 50, 141-147 (2015).

2. Pourier, V. E. C. \& De Borst, G. J. Which carotid artery aneurysms need to be treated (and how)? J. Cardiovasc. Surg. (Torino). 57, 152-157 (2016).

3. Pourier, V. E. C. et al. Experience of a single center in the conservative approach of 20 consecutive cases of asymptomatic extracranial carotid artery aneurysms. Eur. J. Neurol. 1-5 (2018). doi:10.1111/ene.13720

4. Chaikof, E. L. et al. The Society for Vascular Surgery practice guidelines on the care of patients with an abdominal aortic aneurysm. J. Vasc. Surg. 67, 2-77.e2 (2018).

5. Riambau, V. et al. Editor's Choice - Management of Descending Thoracic Aorta Diseases: Clinical Practice Guidelines of the European Society for Vascular Surgery (ESVS). Eur. J. Vasc. Endovasc. Surg. 53, 4-52 (2017).

6. Wanhainen, A. et al. European Society for Vascular Surgery ( ESVS ) 2019 Clinical Practice Guidelines on the Management of Abdominal Aorto-iliac Artery Aneurysms. Eur. J. Vasc. Endovasc. Surg. 1-97 (2019). doi:10.1016/j.ejvs.2018.09.020

7. Garg, K. et al. Presentation and management of carotid artery aneurysms and pseudoaneurysms. J. Vasc. Surg. 55, 1618-1622 (2012).

8. Nordanstig, J., Gelin, J., Jensen, N. \& Osterberg, K. National Experience with Extracranial Carotid Artery Aneurysms : Epidemiology, Surgical Treatment Strategy, and Treatment Outcome. Ann. Vasc. Surg. 28, 882-886 (2014).

9. Welleweerd, J. C. et al. Rationale and design of the extracranial carotid artery aneurysm registry. J Cardiovasc Surg 56, 1-27 (2015).

10. Landis, J. R. \& Koch, G. G. The Measurement of Observer Agreement for Categorical Data. Biometrics 33, 159-174 (1977).

11. Dice, L. R. Measures of the Amount of Ecologic Association Between Species. Ecology 26, 297-302 (1945).

12. Huttenlocher, D., Klanderman, G. \& Rucklidge, W. Comparing images using the Hausdorff distance. IEEE Trans. Pattern Anal. Mach. Intell. 15, 850-863 (1993).

13. Mendrik, A. M. et al. MRBrainS Challenge: Online Evaluation Framework for Brain Image Segmentation in 3T MRI Scans. Comput. Intell. Neurosci. 2015, 813696 (2015).

14. van Keulen, J. W., van Prehn, J., Prokop, M., Moll, F. L. \& van Herwaarden, J. A. Potential value of aneurysm sac volume measurements in addition to diameter measurements after endovascular aneurysm repair. J. Endovasc. Ther. 16, 506-13 (2009).

15. Schnitzbauer, M. et al. CT after Endovascular Repair of Abdominal Aortic Aneurysms: Diagnostic Accuracy of Diameter Measurements for the Detection of Aneurysm Sac Enlargement. J. Vasc. Interv. Radiol. 29, 178-187.e3 (2018).

16. Kontopodis, N. et al. Advances in determining abdominal aortic aneurysm size and growth. World J. Radiol. 8,148 (2016).

17. Dengler, J. et al. Quantifying unruptured giant intracranial aneurysms by measuring diameter and volume-a comparative analysis of 69 cases. Acta Neurochir. (Wien). 157, 361-368 (2015).

18. Ricco, J. B. \& Forbes, T. L. Trans-Atlantic Debate: External Diameter for Abdominal Aortic Aneurysm (AAA) Size Versus Volume. Eur. J. Vasc. Endovasc. Surg. 46, 1 (2013). 


\section{Supplemental Material}

Appendix A: Schematic overview of the twenty cases, and subfield segmentations of each case according to measurement method 2 (the ECAA). Each case consists of two scans performed at different time points, adding to a total of 40 scans.

\begin{tabular}{ll}
\hline Case Method 2A: Fusiform \\
\hline 1
\end{tabular}

2

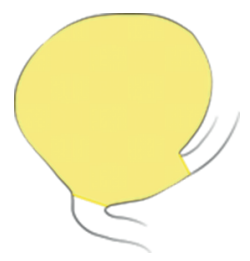

3

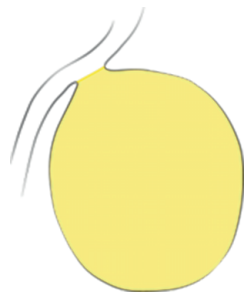

4

5

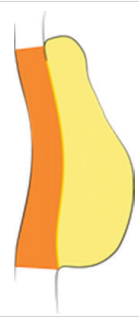



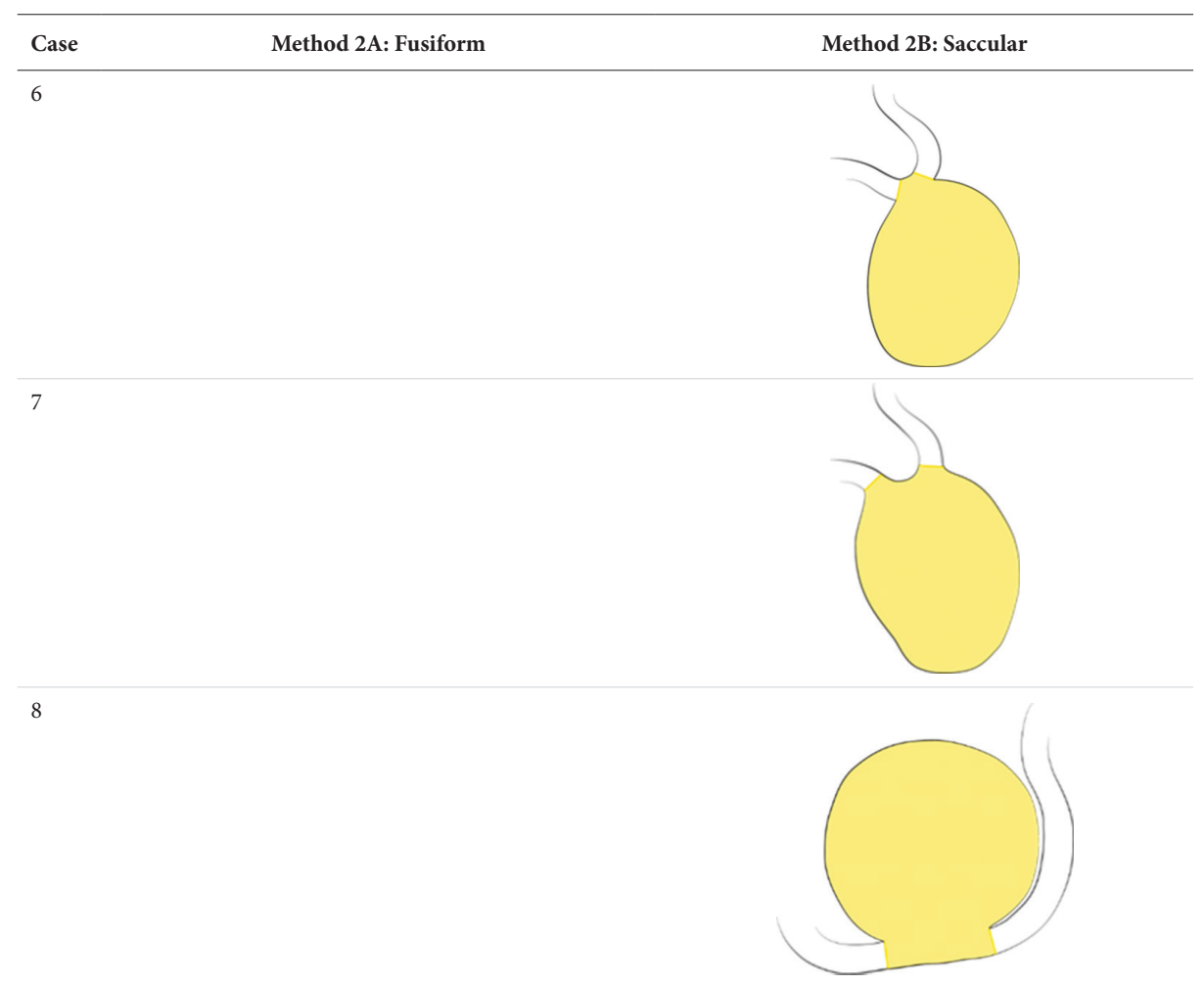

9

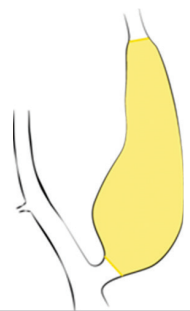

10

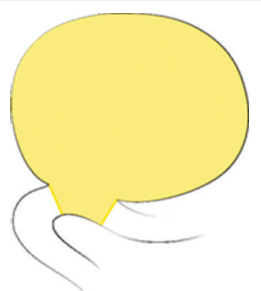

11

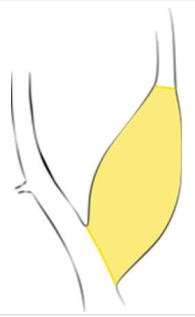




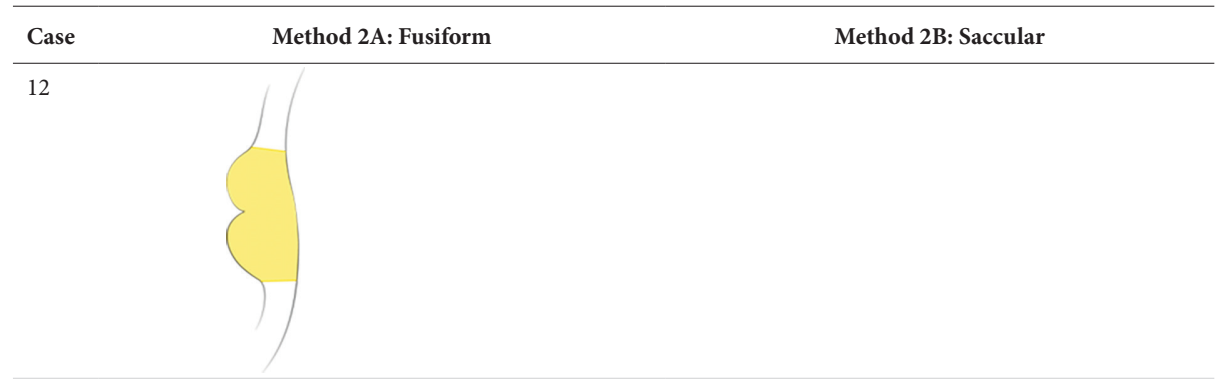

13

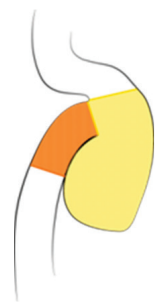

14

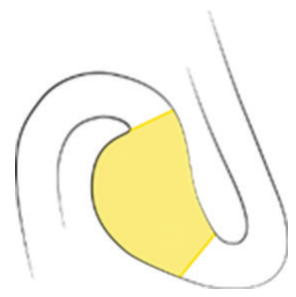

15

16
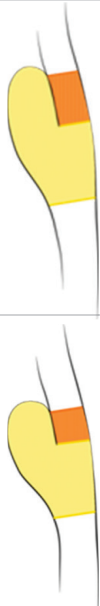


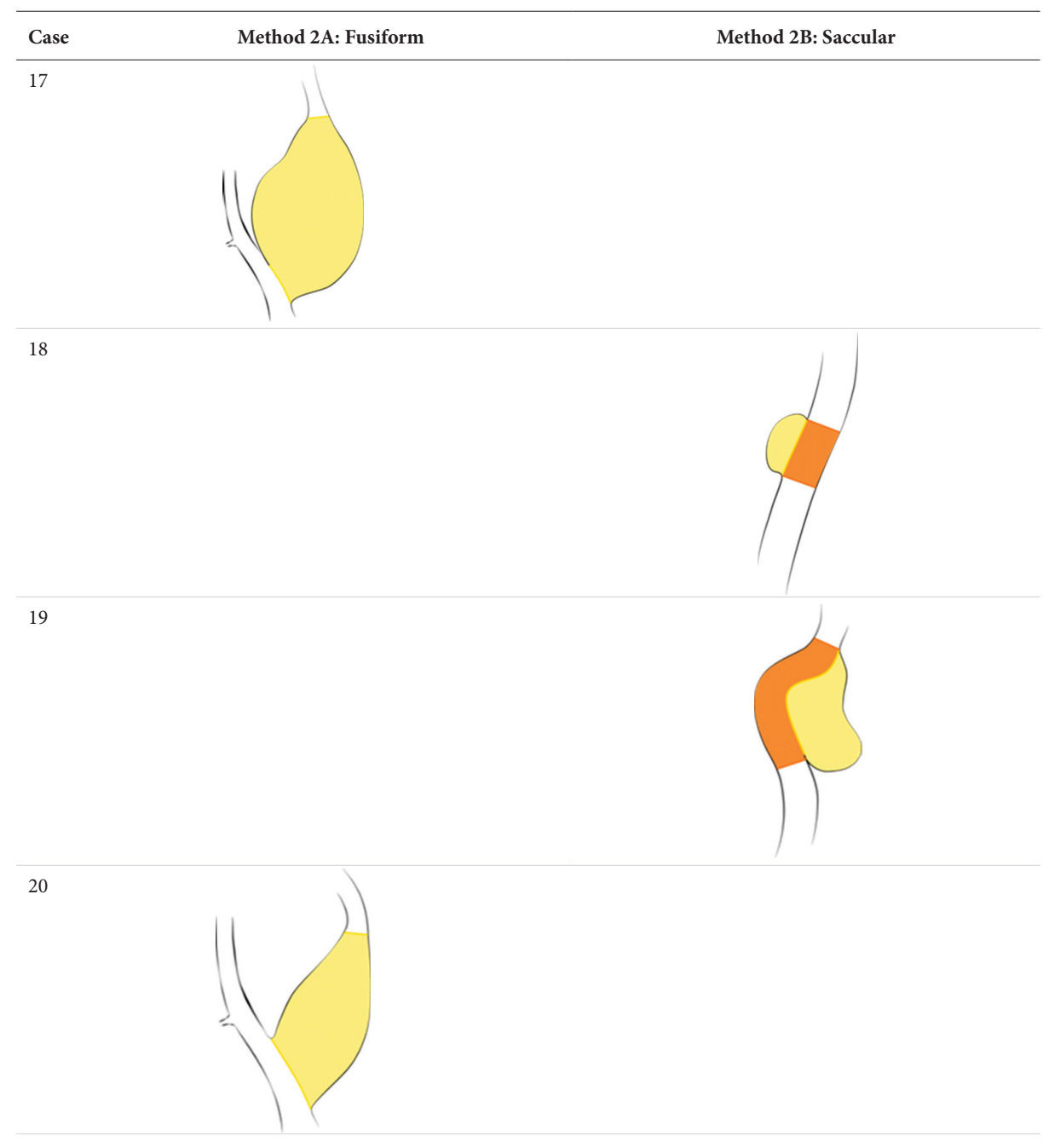




\section{Appendix B}

Final 3D volume segmentations of twenty cases. Each case consists of $a$ and $b$, indicating the paired scans of the same case performed at different time points. In each scan, the segmentation of observer 1 is shown on the left, the segmentation of observer 2 on the right.

Color coding of subfields:

- $\quad$ only vessel = blue.

- aneurysm sac = yellow.

- vessel adjacent to aneurysm sac $=$ orange.
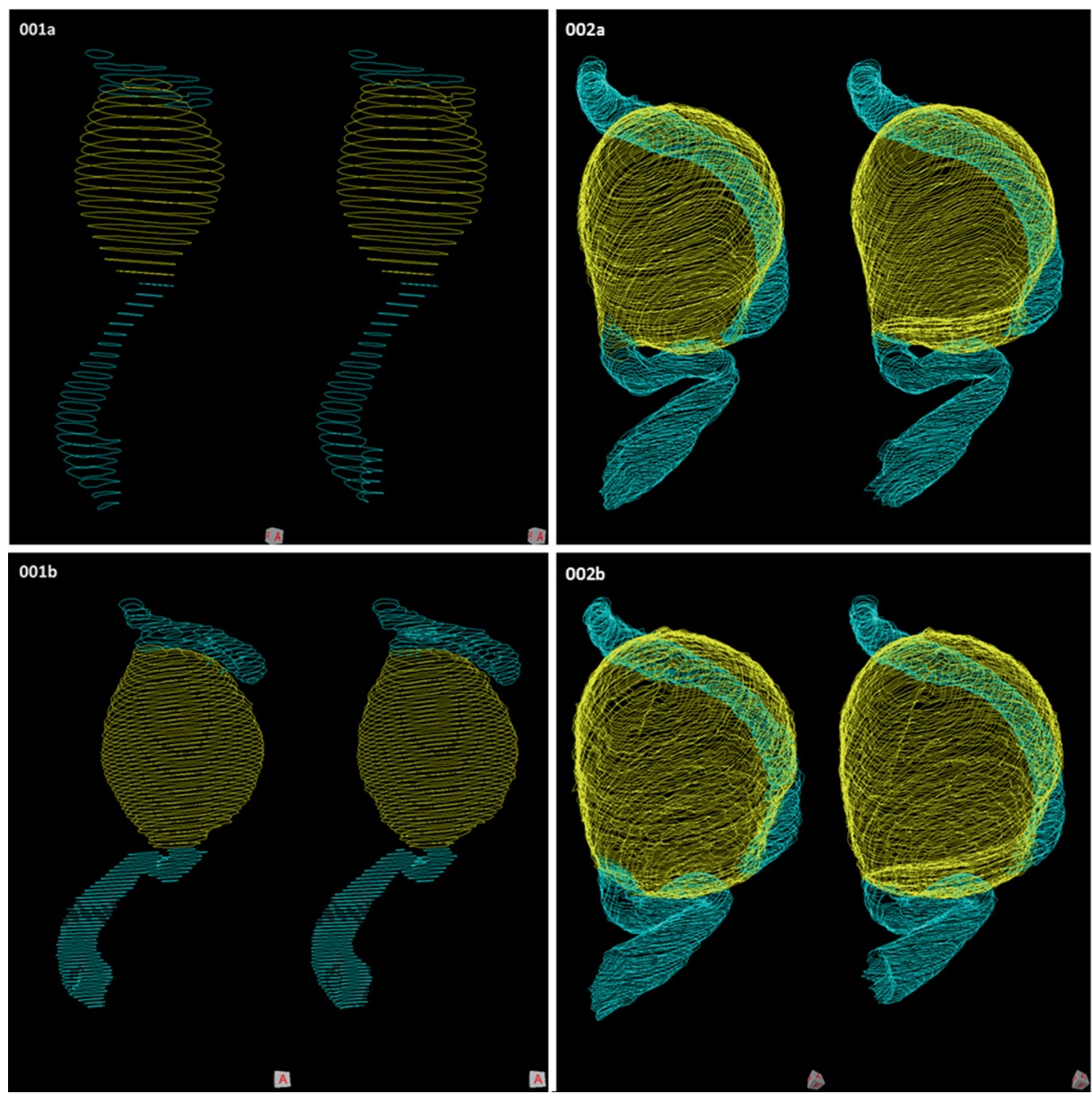

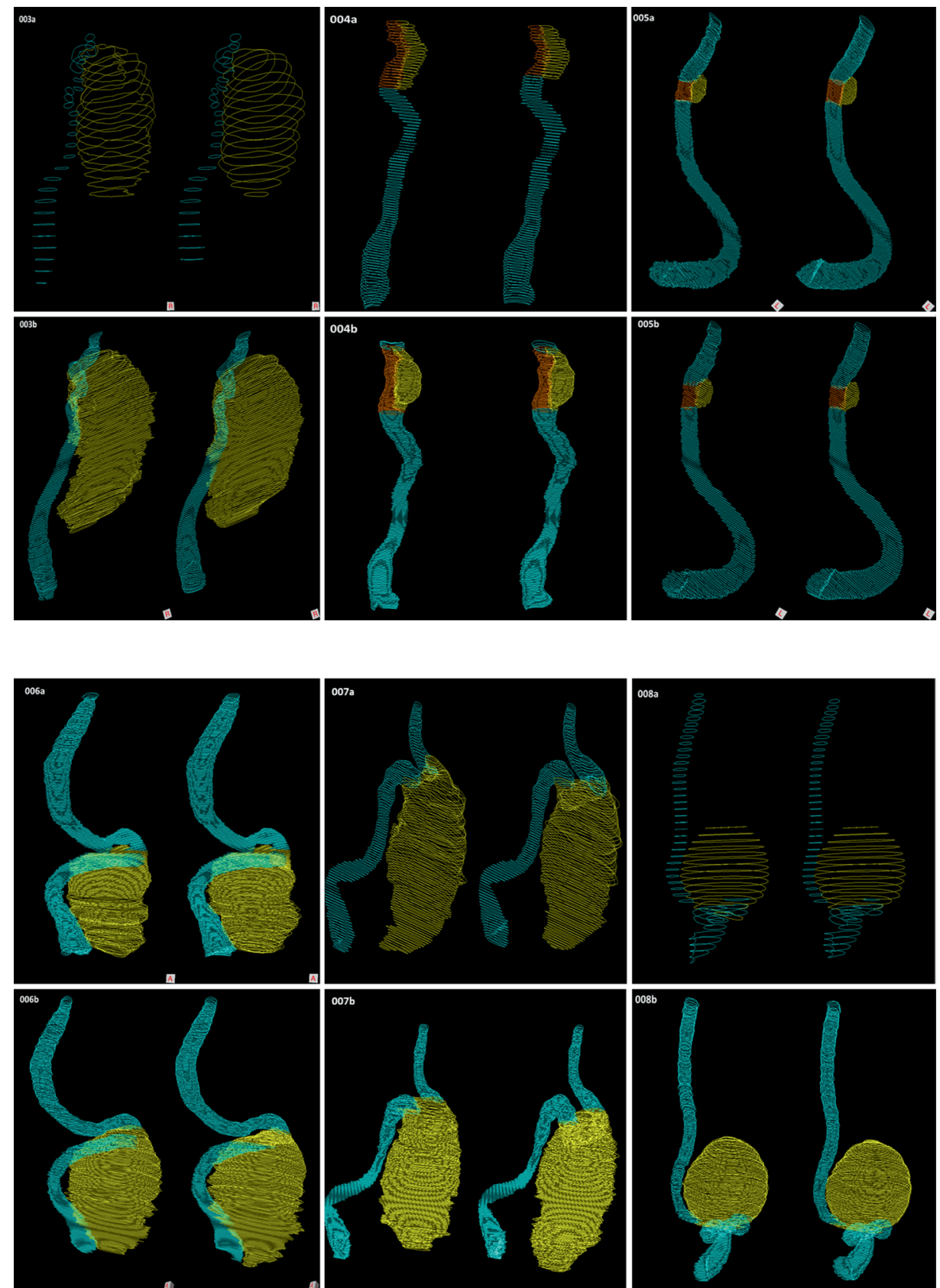


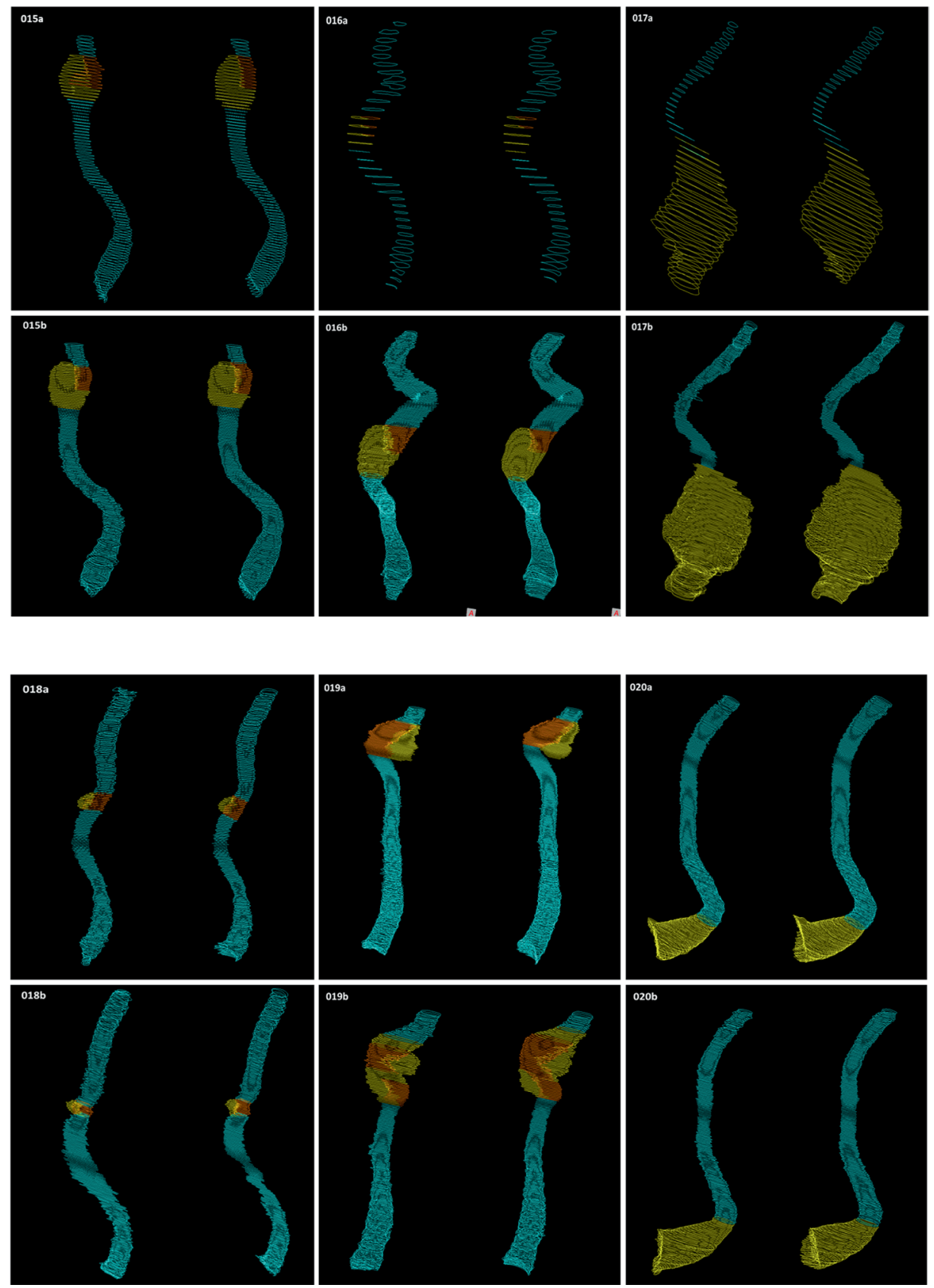


\section{Appendix C}

Supplemental Figure A1-4. The line in the middle represents the mean difference of the volume between the two observers, and the dotted lines represent the upper and lower limits of agreement (mean difference $\pm 1.96 \times$ standard deviation).

Bland-Altman plots showing agreement of two observers on the volume measurement of 40 scans according to the three subfield segmentations, as well as the intraobserver agreement of both observers.

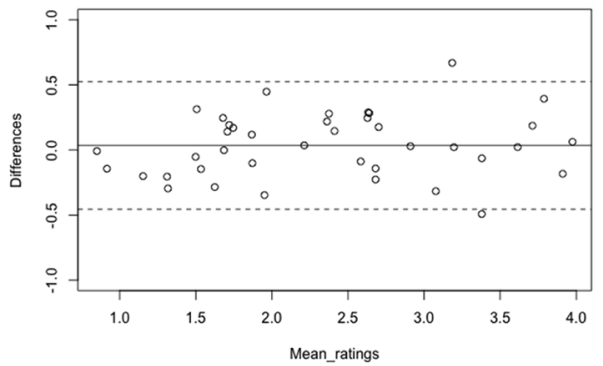

A1. Bland-Altman plot of subfield segmentation "only vessel".

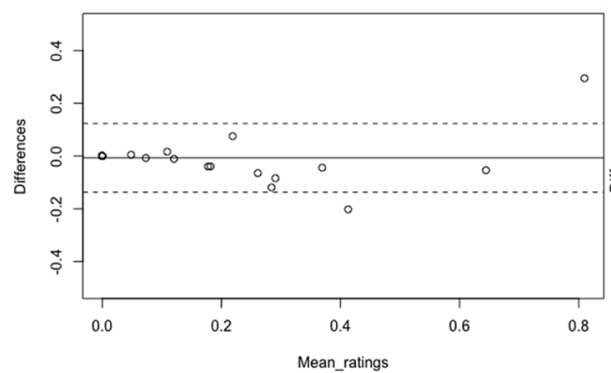

A3. Bland-Altman plot of subfield segmentation "vessel adjacent to aneurysm sac" (top left).

A4. Bland-Altman plots of the intraobserver agreement of measurement method 2 for subset of 5 randomly selected cases, for observer 1 (top right) and observer 2 (bottom right).

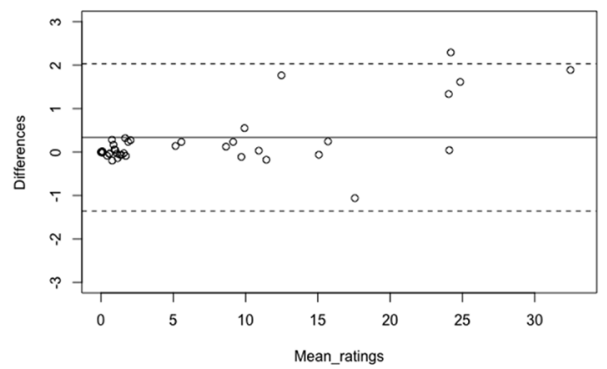

A2. Bland-Altman plot of subfield segmentation "aneurysm sac".
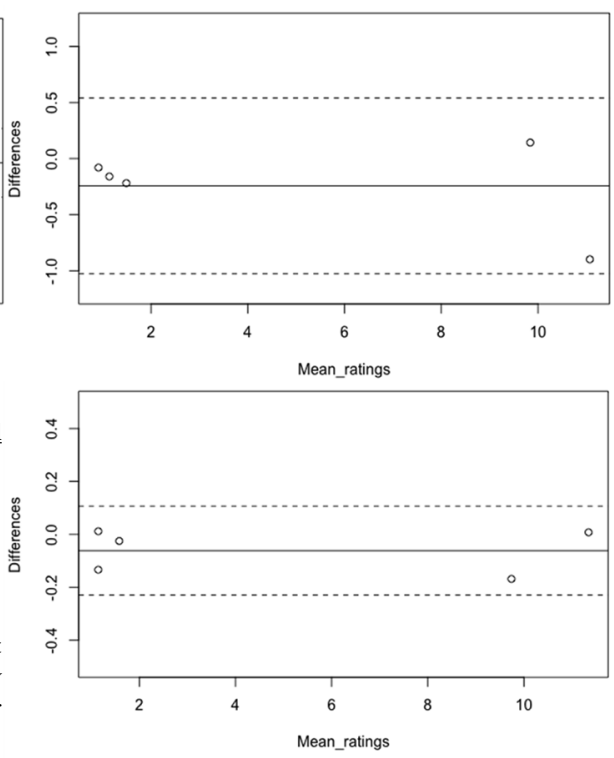



\section{Chapter 8}

\section{Gadolinium enhancement of the aneurysm wall in extracranial carotid artery aneurysms}

Am J Neuroradiology 2020;41:501-507.

C.J.H.C.M. van Laarhoven*

M.L. Rots*

V.E.C. Pourier

N.K.N. Jorritsma

T. Leiner

J. Hendrikse

M.D.I. Vergouwen

G.J. de Borst

* Shared first authorship 


\section{Abstract}

\section{Background and purpose}

The natural history and optimal treatment of extracranial carotid artery aneurysms (ECAAs) are unknown. Gadolinium-enhancement of the aneurysm wall may reflect aneurysm wall inflammation and instability. In this study we investigated the feasibility of ECAA wall imaging and explored a potential relationship of aneurysm wallenhancement with aneurysm growth and presence of (silent) brain infarcts and WMLs.

\section{Methods}

Fourteen conservatively treated patients with fifteen asymptomatic ECAAs underwent gadolinium-enhanced 3T-MRI at two time-points with a 12-month interval. Primary outcome was growth of the aneurysm sac $(\geq 2.0 \mathrm{~mm})$, secondary outcomes were presence of (silent) brain infarcts and white matter lesions (WMLs) at baseline and follow-up. MR-images were reviewed by two independent observers and inter- and intraobserver reproducibility was assessed.

\section{Results}

Seven (50\%) patients were male; median age was 55 years (range 40-69). Eleven ECAAs (73\%) were saccular (median size $11 \mathrm{~mm}$, range 5.0-38.5) and four were fusiform (median size $21.5 \mathrm{~mm}$, range 10.0-40.0). Eleven out of fifteen aneurysms (73\%) exhibited gadolinium-enhancement $(\mathrm{Gd}+)$ at baseline. Four aneurysms (27\%) showed growth at follow-up imaging, two Gd+and two Gd- ( $p=.245)$. Three patients (21\%) had ipsilateral brain infarcts at baseline, one of them showed new silent brain infarct at follow-up imaging $(\mathrm{Gd}+)$. Nine patients (64\%) showed bilateral WMLs at baseline. In three patients increased WML severity was observed at follow-up (two Gd+). All observations showed excellent inter- and intraobserver reproducibility.

\section{Conclusion}

In this explorative study we demonstrated that ECAA wall-imaging was feasible. Future well-powered studies are needed to investigate if ECAA gadolinium-enhancement predicts aneurysm growth and thrombo-embolic complications. 


\section{Introduction}

The optimal treatment and prognosis of extracranial carotid artery aneurysms (ECAAs) are still unclear. Most ECAAs are coincidental findings and a conservative approach is presently considered justified in patients with asymptomatic ECAA ${ }^{1}$. Several surgical and endovascular techniques have been developed for ECAA exclusion, but these interventions are mostly reserved for patients with neurological symptoms or with proven growth $^{2,3}$. Histopathological research revealed dissection and degeneration as the two main causes of ECAA, with the presence of inflammatory cells in the degenerative vessel wall ${ }^{4}$. MRI and histopathological studies of atherosclerotic carotid arteries have suggested that gadolinium $(\mathrm{Gd})$-enhancement reflects the density of vasa vasorum and inflammation in the arterial wall ${ }^{5,6}$. Also, MRI has been proposed as a non-invasive tool for early detection of arterial wall changes and may be useful for routine monitoring and evaluation of disease activity ${ }^{6}$. However, ECAA growth pattern and long-term risk of thromboembolization and subsequent stroke has yet to be elucidated. Previous studies have shown that patients with carotid stenosis have (subclinical) microvascular cerebral damage due to micro-embolic events ${ }^{7}$. Silent brain infarction and white matter lesions (WMLs) have both been correlated with carotid plaque vulnerability and thus may be a reflection of micro-embolic events from a proximal source ${ }^{8,9}$. Silent brain infarcts and WML presence has been described to correlate with increased future risk of ischemic stroke and cognitive decline over time ${ }^{10-12}$. Assessment of both silent and symptomatic brain infarcts and WMLs may therefore be helpful surrogate markers for cerebral outcome in ECAA patients.

New imaging techniques are required to assess ECAA instability in order to predict growth and cerebrovascular outcome. In this study of patients with conservatively treated asymptomatic ECAA, we aimed to investigate the feasibility of ECAA wall imaging and explored a potential relationship of aneurysm wall-enhancement with aneurysm growth and presence of (silent) brain infarcts and WMLs.

\section{Methods}

\section{Participants}

Ethical approval for this study was provided by the Medical Research Ethics Committee of University Medical Center Utrecht, Utrecht, The Netherlands on 13 September 2016 (protocol number 16-341), and all research was conducted according to the principles of the Declaration of Helsinki (59th amendment, Seoul 2008) and in accordance with the Dutch Medical Research Involving Human Subjects Act (WMO). Included patients 
with an ECAA were selected from the Carotid Aneurysm Registry (CAR, www. carotidaneurysmregistry.com). The registry protocol has been published elsewhere ${ }^{13}$. In short, any patient aged 18-years or older with an ECAA is included in this ongoing registry, independent of etiology or treatment strategy. Baseline characteristics and follow-up data are collected in a prospective manner. For the present study, we selected asymptomatic patients (no symptoms for at least 1 year prior to the start of the study) from the CAR with a conservatively treated ECAA. Exclusion criteria were impaired renal function (glomerular filtration rate $<30 \mathrm{~mL} / \mathrm{min} / 1.73 \mathrm{~m}$ ), Gd contrast allergy, or pregnancy. All selected patients provided informed consent and were recruited between February and September 2017.

\section{MR imaging}

Included patients were asked to undergo MRI at moment of inclusion and one year after. Two observers (C.L., M.R.) blinded to patient characteristics and previous imaging, independently scored each of the MR scans according to a predefined protocol and case report form. Prior to scoring, both observers were trained with two test scans to familiarize with the scoring protocol. Test results were compared to overcome interpretation differences. Aneurysm location, shape and size, vessel wall-enhancement and cerebral complications were reported for two series of MR images (baseline and follow-up), with a minimum of two weeks between the measurements. For a subset of MR images, a third series was reevaluated after two weeks to determine intraobserver variability. For the final data analysis, disagreements were discussed until consensus was reached.

A 3.0 Tesla MR scanner (Ingenia, Software release 5.3, Philips Healthcare, Eindhoven, The Netherlands) was used. Images were acquired with an 18-element neurovascular coil as receiver coil. The MRI protocol consisted of a 3-D time-of-flight sequence, a contrast-enhanced T1 3-D sequence (spatial resolution $0.5 \times 0.5 \times 0.5 \mathrm{~mm}$ ), and for imaging of the vessel wall a transverse T1 3D multishot spin-echo sequence of the ipsi- and contralateral extracranial carotid artery with the following parameters: field of view of $200 \times 166 \times 45 \mathrm{~mm}$ (by default but coverage could be adjusted in case of aneurysms $>45 \mathrm{~mm}$ ), acquired resolution $0.5 \times 0.5 \times 1.0 \mathrm{~mm}$, reconstructed resolution $0.5 \times 0.5 \times 0.5 \mathrm{~mm}, \mathrm{TR} / \mathrm{TE}$ $1500 / 38$. An anti-DRIVen Equilibrium technique and a minimum flip angle of $25^{\circ}$ in the variable flip angle refocusing pulse train were used for increased vessel wall contrast, fat suppression and flow suppression of blood ${ }^{14,15}$. This sequence was done before and after contrast administration with a duration of $8 \mathrm{~m} 3 \mathrm{~s}$ per sequence. FLAIR and T2 brain imaging series were performed for detection of infarcts and WMLs. Gadobutrol was used as contrast agent (Gadovist, Bayer Healthcare, Berlin, Germany, $0.1 \mathrm{~mL} / \mathrm{kg}(1 \mathrm{mmol})$ per kilogram body weight), flow rate $1.0 \mathrm{~mL} / \mathrm{sec}$, time between contrast administration and vessel wall imaging was 11 minutes. 


\section{Aneurysm size assessment}

Aneurysm size was determined on the contrast enhanced T1-weighted images using double oblique multiplanar reconstructions, in which maximum diameters were measured. Diameters were rounded to $0.5 \mathrm{~mm}$. Fusiform or spindle-shaped ECAA was defined as $\geq 150 \%$ dilation of the arterial diameter, compared to the non-affected contralateral carotid artery diameter. In case of bilateral dilatation, the diameter of the non-affected part of the ipsilateral carotid artery was used as comparison. For saccular shaped ECAA all sizes were accepted ${ }^{1}$. In case of saccular type, length, width, and neck of the aneurysm was reported, in case of fusiform type length and width were reported (Figure 1). It was decided to define growth as a minimum difference of at least $2.0 \mathrm{~mm}$ measured in at least one of the measured dimensions (length, width, and neck), to ensure reliable assessment of growth at the acquired resolution $(0.5 \times 0.5 \times 0.5 \mathrm{~mm})$. We considered differences of $<1.0 \mathrm{~mm}$ in ECAA size clinically irrelevant.
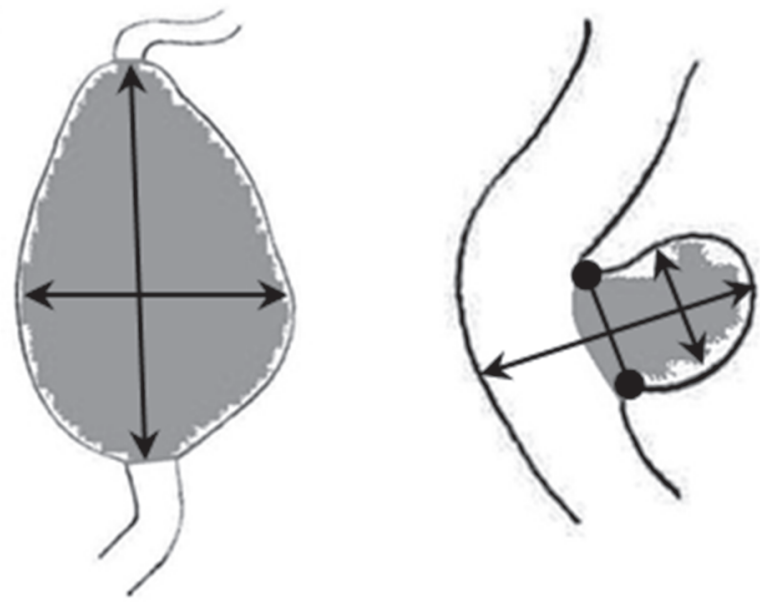

Figure 1. Schematic examples of diameter measurements of ECAA, perpendicular to the non-affected vessel. Left: fusiform ECAA length $\mathrm{x}$ width, right: saccular ECAA length $\mathrm{x}$ width $\mathrm{x}$ neck (round arrow).

\section{Assessment of Gd ECAA wall-enhancement}

The appearance of the aneurysms was compared before and after administration of Gd to determine the presence of wall-enhancement. Aneurysm wall-enhancement was considered present if there was a hyperintensity of the vascular wall on the MRI after Gd administration, that was not present on the MRI before Gd administration (Figure 3). Ipsilateral aneurysm wall-enhancement was reported as well as contralateral enhancement of the internal carotid artery at the level of the aneurysm as a reference. 


\section{Assessment of brain infarcts and WML}

Presence of cortical, subcortical and lacunar infarcts on ipsi- or contralateral side was reported $^{16}$, and expressed as symptomatic or silent brain infarction. WMLs were semi quantitatively assessed at baseline and follow-up using the Fazekas scale ${ }^{17,18}$. In short, WMLs were separated according to whether hyperintense lesions were contiguous with the lateral ventricular border (periventricular lesion) or distinct and subcortical (including those in the deep white matter). Subcortical extensions from cortical and lacunar infarcts were excluded.

\section{Statistical analysis}

All observations were assessed in terms of reliability and agreement. Inter- and intraobserver reliability of each diameter was calculated by the intraclass correlation coefficient (ICC; model: two-way mixed, type: absolute agreement) with 95\% confidence interval (CI). Bland-Altman analysis was used to assess agreement for each diameter. For nominal and binary variables (aneurysm location, shape, Gd-enhancement, and infarcts) Kappa-values were calculated, and for ordinal variables (WML) weighted Kappa was calculated ${ }^{19}$. 95\% CI for Kappa were obtained by use of package ' $p s y$ ' and 'boot' (http:// cran.r-project.org) in Rstudiov3.4.1 (RStudio Team (2016). RStudio: Integrated Development for R. RStudio, Inc., Boston, MA, www.rstudio.com). Proportion of agreement was calculated for both nominal and ordinal variables ${ }^{20}$.We studied the proportion of patients with aneurysm wall-enhancement. Then, we investigated whether aneurysm wall-enhancement was associated with signs of instability during follow-up. The primary outcome was the prevalence of aneurysm wall-enhancement. Secondary outcomes were growth of the aneurysm sac during follow-up, and the presence of WMLs and (silent) brain infarcts at baseline and follow-up. Patients were compared based on Gd-enhancement by use of Fisher's Exact test and Mann Whitney $U$ test and $p<.05$ was considered statistically significant. These statistical analyses were conducted using SPSS v25.0 (IBM Corp. Released 2017. IBM SPSS Statistics for Windows, Version 25.0. Armonk, NY: IBM Corp.).

\section{Results}

Fifteen patients with 16 ECAAs were included and underwent baseline MRI. Fourteen patients (with fifteen aneurysms) underwent follow-up MRI after one year. Individual patient characteristics are listed in Table 1. The patient that was lost to follow-up was unable to participate in study activities due to clinical deterioration as a result of prior cerebral infarction. Eight out of fifteen patients (53\%) were male; median age was 55 years (range 40-69). Detailed medical history is summarized in Supplemental Table I. 


\begin{tabular}{|c|c|c|c|c|c|c|c|c|c|c|c|c|}
\hline & & & \multicolumn{5}{|c|}{$\begin{array}{l}\text { Baseline } \\
\text { Ipsilateral }\end{array}$} & \multicolumn{5}{|c|}{$\begin{array}{c}1 \text { year after baseline } \\
\text { Ipsilateral }\end{array}$} \\
\hline $\begin{array}{l}\stackrel{5}{\infty} \\
\dot{1} \\
\dot{\omega} \\
\tilde{\omega}\end{array}$ & 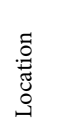 & $\begin{array}{l}\frac{1}{2} \\
\frac{0}{2} \frac{0}{0} \\
0\end{array}$ & 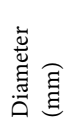 & 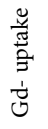 & 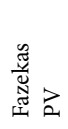 & 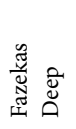 & 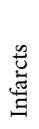 & 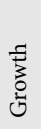 & 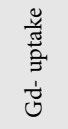 & 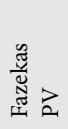 & 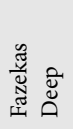 & 䒕 \\
\hline M-49 & LICA & S & 5.0 & $\bullet$ & 0 & 0 & $\circ$ & $\circ$ & • & 0 & 0 & $\circ$ \\
\hline M-51 & RICA & $S$ & 11.0 & $\bullet$ & 0 & 0 & $\bullet$ & $\circ$ & $\bullet$ & 1 & 0 & $\bullet$ \\
\hline F-66 & LICA & S & 11.0 & $\circ$ & 2 & 1 & $\circ$ & $\circ$ & $\bullet$ & 2 & 2 & 0 \\
\hline M-41 & RICA & $\mathrm{F}$ & 19.0 & $\bullet$ & 0 & 0 & $\circ$ & $\circ$ & $\bullet$ & 0 & 0 & $\circ$ \\
\hline F-51 & LICA & $S$ & 7.5 & $\bullet$ & 0 & 0 & $\circ$ & $\circ$ & $\bullet$ & 0 & 0 & $\circ$ \\
\hline F-66 & RICA & $S$ & 38.5 & $\bullet$ & 3 & 3 & $\bullet$ & $\bullet$ & $\bullet$ & 3 & 3 & $\bullet$ \\
\hline F-59 & LICA & $\mathrm{F}$ & 10.0 & $\bullet$ & 1 & 0 & $\circ$ & $\circ$ & ○ & 1 & 2 & $\circ$ \\
\hline M-54 & RICA & S & 9.0 & $\circ$ & 0 & 0 & $\circ$ & $\bullet$ & $\circ$ & 0 & 0 & $\circ$ \\
\hline M-69 & RICA & S & 13.0 & $\bullet$ & 2 & 2 & $\circ$ & $\bullet$ & ? & 2 & 2 & $\circ$ \\
\hline M-52 & LICA & $S$ & 9.5 & $\circ$ & 1 & 2 & $\circ$ & $\circ$ & $\circ$ & 1 & 2 & $\circ$ \\
\hline F-56 & LICA & $S$ & 11.5 & $\bullet$ & 0 & 0 & $\bullet$ & $\circ$ & $\mathrm{N} / \mathrm{A}^{\mathrm{a}}$ & 0 & 0 & $\bullet$ \\
\hline F-40 & RICA & $\mathrm{F}$ & 24.0 & $\bullet$ & 1 & 0 & $\circ$ & $\circ$ & • & 1 & 0 & $\circ$ \\
\hline F-53 & RICA & $\mathrm{F}$ & 40.0 & $\circ$ & 3 & 3 & $\circ$ & $\bullet$ & ० & 3 & 3 & $\circ$ \\
\hline M-55* & RICA & S & 8.5 & $\bullet$ & 1 & 0 & $\bullet$ & & & & & \\
\hline$M-62^{b}$ & LICA & S & 10.5 & $\bullet$ & 2 & 2 & $\circ$ & $\circ$ & $\bullet$ & 2 & 2 & $\circ$ \\
\hline$M-62^{b}$ & RICA & S & 11.0 & $\bullet$ & 1 & 1 & $\circ$ & $\circ$ & $\bullet$ & 1 & 1 & $\circ$ \\
\hline
\end{tabular}

Abbreviations: $\mathrm{M}=$ male, $\mathrm{F}=$ female, $\mathrm{LICA}=$ left internal carotid artery, $\mathrm{RICA}=$ right internal carotid artery, $\mathrm{S}=$ saccular, $\mathrm{F}$ = fusiform, $\mathrm{mm}=$ millimeter, $\mathrm{Gd}=$ gadolinium, $\mathrm{N} / \mathrm{A}=$ Not available, $\mathrm{PV}=$ periventricular, $\mathrm{FU}=$ follow-up. Diameter is given for aneurysm width in $\mathrm{mm}$. Fazekas score is the highest ipsilateral score.

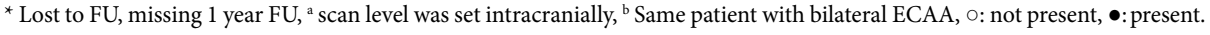

All MR observations showed excellent inter- and intraobserver reproducibility, with both ICCs and weighted Kappa's > 0.9, and acceptable differences for agreement (Figure 2, Supplemental Table II, Supplemental Figure I-VIII). In total, 666 observations per observer were made of which $15 \%$ disagreements were discussed.

\section{Aneurysm analysis}

All sixteen ECAAs were located in the extracranial internal carotid artery (eight on the right, six on the left and one patient with bilateral ECAA). Twelve aneurysms were saccular (75\%) and four were fusiform. Median length was $13.5 \mathrm{~mm}$ (range 6.5-66.0mm), and median width was $11.5 \mathrm{~mm}$ (range $5.5-41.0 \mathrm{~mm}$ ). The median neck width was $11 \mathrm{~mm}$ (3.5-22.0mm) in saccular ECAAs. Twelve out of sixteen (75\%) aneurysms had Gd wallenhancement at baseline. Of the fifteen aneurysms with follow-up imaging available, four $(27 \%)$ increased in size $\geq 2.0 \mathrm{~mm}$ in at least one direction at the follow-up MRI. 


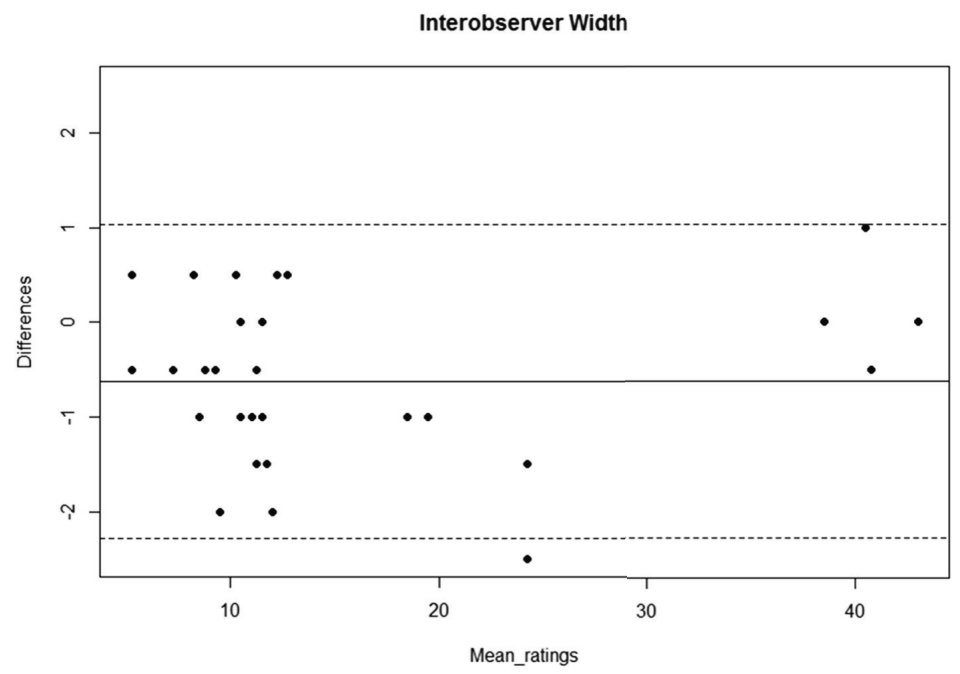

Figure 2. Bland-Altman plot of interobserver agreement. The solid line in the middle represents the mean difference of the diameter measurement between the two observers, the dashed line represents the upper and lower limits of agreement (mean difference $\pm 1.96 \mathrm{x}$ standard deviation).

Of these four aneurysms, one showed growth in length, width and neck, one showed only increased width, and two showed only increase in length in comparison to baseline. For further analysis, only patients with available follow-up MRA are included.

\section{Aneurysm wall Gd-enhancement}

All 28 aneurysm wall scans showed good image quality and no artifacts interfered with our assessment of wall enhancement. Out of the fifteen aneurysms with follow-up imaging, eleven exhibited ECAA wall-enhancement at baseline. Length, width and neck diameter of aneurysms with wall-enhancement compared to those without was similar (see Table 2). Of the four aneurysms that increased in size at follow-up, two (50\%) showed aneurysm wall Gd-enhancement on baseline MRI (see Table 2). When absolute differences between baseline and follow-up MRI were compared, the median increase in neck size (only saccular aneurysms) was larger in the eight $\mathrm{Gd}+$ (median $2.0 \mathrm{~mm}$; range 0.5-3.0) compared to the three Gd- ECAAs (median 0.0mm; range -1.0-0.5, $p=.012$ ). Other differences over time (length and width) were comparable in patients with and without aneurysm wall-enhancement (Table 2). A total of three patients showed wall-enhancement of the contralateral carotid artery at the level of the aneurysm, all of whom also had ipsilateral enhancement of the ECAA wall. Upon follow-up, 9/15 aneurysms showed steady ipsilateral wall-enhancement and 3/15 aneurysms showed consistently no enhancement. One Gd+ aneurysm at baseline did no longer show enhancement at followup and one Gd-patient at baseline showed novel enhancement at follow-up whereas this 


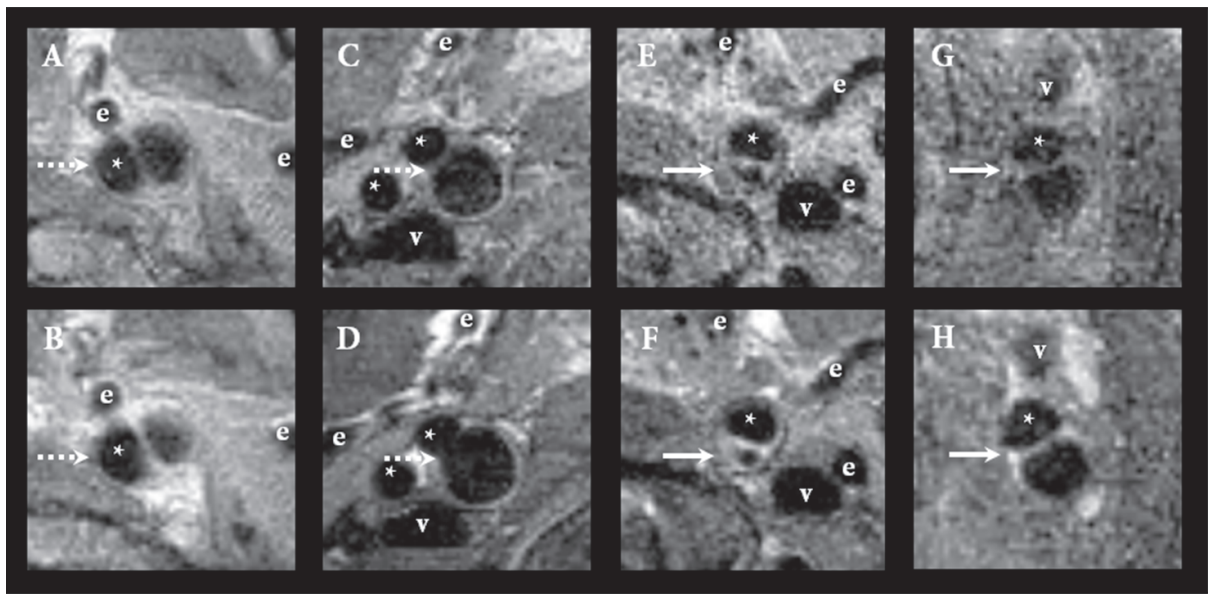

Figure 3. Examples of aneurysm wall enhancement on 3T MRI with aneurysm wall imaging on trans-axial plane. B,D,F, and $\mathbf{H}$ show the sequence after administration of gadolinium. A and $\mathbf{B}$ show a 66-yr-old woman with a 11.0mm saccular LICA aneurysm without enhancement (dashed arrow). $\mathbf{C}$ and $\mathbf{D}$ show a 54-yr-old man with a $9.0 \mathrm{~mm}$ saccular RICA aneurysm without enhancement (dashed arrow). E and F show a 51-yr-old woman with a $7.5 \mathrm{~mm}$ saccular LICA aneurysm with aneurysm enhancement (solid arrow). $\mathbf{G}$ and $\mathbf{H}$ show a 59 -yr-old woman with a $10.0 \mathrm{~mm}$ fusiform LICA aneurysm with enhancement (solid arrow). Abbreviations: LICA = left internal carotid artery, RICA = right internal carotid artery, ${ }^{*}$ indicates parent ICA, $\mathrm{e}=$ branches of the external carotid artery, $\mathrm{v}=$ internal jugular vein.

was not present as baseline. For the remaining patient follow-up enhancement could not be evaluated, as the scan level was set intracranially.

\section{Assessment of brain infarcts and WML}

At baseline, two patients showed lacunar (silent) brain infarcts, one ipsilateral and one patient with multiple bilateral lacunar brain infarcts (Figure 4). Two other patients had cortical infarcts, one of them ipsilateral to the aneurysm and another patient with a cortical infarct contralateral to the aneurysm. In both patients cortical infarcts were clinically symptomatic, though $>1$ year prior to start of the study. No subcortical infarcts were observed and none of the infarcts were located in the area of the posterior circulation. Follow-up MRI revealed that the patient who showed multiple bilateral lacunar infarcts at baseline had a bilateral increase of the number of lacunar silent brain infarcts on follow-up imaging. Another patient had one new contralateral lacunar silent brain infarct. All patients with infarcts at baseline or follow-up showed Gd-enhancement at baseline. The median Fazekas score at baseline was 1 (range 0-3) for ipsilateral periventricular WML and 1 for contralateral periventricular WML (range 0-3, $p=.705$ ). Median Fazekas score for deep WML was 1 (range 0-3) at the site ipsilateral to the aneurysm and 0 (range $0-3)$ on the contralateral site $(p=.816)$. Three out of fourteen patients with follow-up imaging showed an increase of Fazekas score after one year, of whom two on the ipsilateral site (one patient with increase of deep WML and one patient with increase of 
Table 2. Aneurysm size for aneurysms with and without gadolinium enhancement.

\begin{tabular}{lccc}
\hline & $\begin{array}{c}\text { No gadolinium enhancement } \\
(\mathbf{n}=\mathbf{4})\end{array}$ & $\begin{array}{c}\text { Gadolinium enhancement } \\
(\mathbf{n = 1 2})\end{array}$ & $\boldsymbol{p}$ \\
\hline $\begin{array}{l}\text { Length baseline } \\
\text { Median (range) }\end{array}$ & $10.75(6.5-46.0)$ & $14.5(7.00-66.5)$ & 0.316 \\
$\begin{array}{l}\text { Width baseline } \\
\text { Median (range) }\end{array}$ & $10.5(9.0-41.0)$ & $11.5(5.5-38.5)$ & 0.953 \\
\hline $\begin{array}{l}\text { Neck baseline } \\
\text { Median (range) }\end{array}$ & $9.5(8.0-11.0)$ & $12.0(3.5-22.0)$ & 0.482 \\
$\begin{array}{l}\text { Growth (length) } \\
\text { Median (range) }\end{array}$ & $1.0(-1.0-4.0)$ & $0.5(-0.5-4.5)$ & $>0.999$ \\
\hline $\begin{array}{l}\text { Growth (width) } \\
\text { Median (range) }\end{array}$ & $1.0(-1.0-4.0)$ & $0.5(-0.5-4.5)$ & $>0.999$ \\
\hline $\begin{array}{l}\text { Growth (neck) } \\
\text { Median (range) }\end{array}$ & $0.0(-1.0-0.5)$ & $2.0(0.5-3.0)$ & 0.012 \\
\hline $\begin{array}{l}\text { Growth (any direction } \geq \mathbf{2 m m}) \\
n \text { (\%) }\end{array}$ & $2(50)$ & $2(16.7)$ & 0.245 \\
\hline
\end{tabular}

${ }^{a}$ Only saccular aneurysm patients. Bold values indicate $p<.05$.

periventricular WML) and one patient with a bilateral increase of deep WMLs. Two out of three patients who showed an increase of WML had Gd-enhancement at baseline imaging.

\section{Discussion}

In this explorative imaging study we demonstrated that ECAA wall imaging was feasible with good inter- and intraobserver reliability and agreement. The majority of aneurysms had Gd-enhancement of the ECAA wall at baseline. Aneurysm growth occurred in 27\% of all aneurysms.

No earlier studies investigated wall-enhancement of extracranial carotid aneurysms. Only patients with Gd-enhancement of atherosclerotic plaques in the carotid arteries are described $^{21-23}$. It is believed that Gd-enhancement reflects both density of the vasa vasorum and endothelial permeability, and can indicate local inflammation of the vessel wall ${ }^{21-23}$. These previously performed studies used $1.5 \mathrm{~T}^{21,23}$ or $3.0 \mathrm{~T}^{22} \mathrm{MR}$ scanners, with double molality concentration of Gd and flow rate. The current scanning protocol was based on earlier studies investigating intracranial vessel wall imaging and reporting good image quality and little artifacts ${ }^{14}$. Fine-tuning of the optimal imaging protocol for the carotid wall specifically, including the used sequences to suppress artefacts due to blood flow, contrast agent concentration, flow rate and time between administration and vessel 


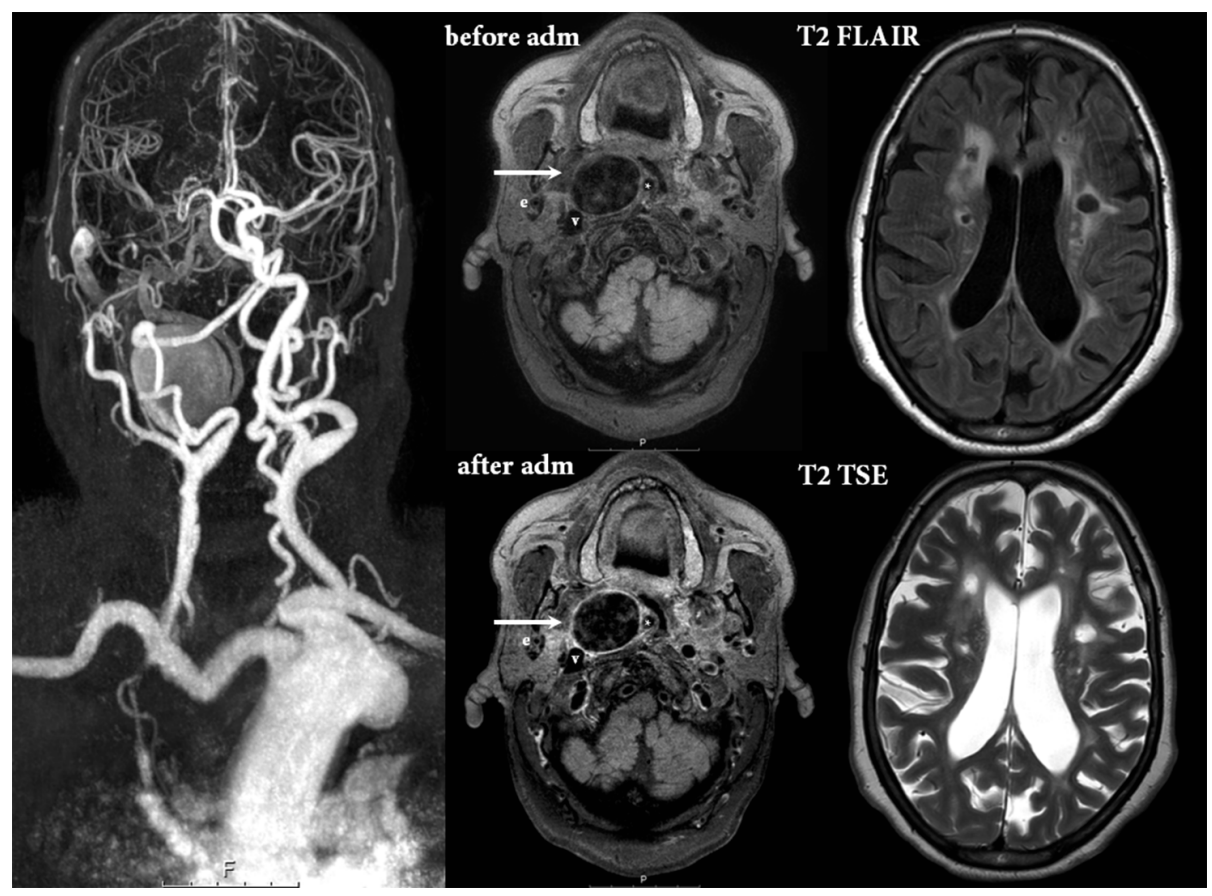

Figure 4. Overview baseline $3 \mathrm{~T}$ Gd-enhanced MRI of 66-yr-old female with a saccular ECAA of the RICA. At baseline enhancement of the aneurysm wall was observed after contrast administration, indicated by the white arrows. T2 FLAIR \& T2 TSE showed both periventricular and deep white matter lesions Fazekas 3 and multiple bilateral lacunar infarctions. Abbreviations: $\mathrm{Gd}=$ gadolinium, adm = administration, $\mathrm{ECAA}=$ extracranial carotid artery aneurysm, RICA = right internal carotid artery, * indicates parent RICA, $\mathrm{e}=$ branches of the right external carotid artery, $\mathrm{v}=$ right internal jugular vein.

wall imaging, is warranted so that uniform reporting standards can be established and study results can be compared.

A follow-up study that included 65 intracranial aneurysms with a median follow-up of 27 months (range 20-31) showed that 4 out 19 aneurysms with wall-enhancement at baseline had instability during follow-up, compared to 0 out of 46 aneurysms without wallenhancement ${ }^{24}$. Although our study in patients with ECAAs was not powered to detect differences in aneurysm growth between patients with and without aneurysm wallenhancement, our follow-up results are of interest since we also found aneurysm growth in 2 out of 4 patients without wall-enhancement. Since healthy intracranial arteries lack adventitial vasa vasorum ${ }^{25,26}$, Gd-enhancement of intracranial arteries may indicate stronger local pathological neovascularization and thus inflammation than in extracranial carotid arteries ${ }^{26}$. Moreover, the discrimination between moderate or major enhancement in extracranial arteries, possibly indicating pathological neovascularization of the aneurysm wall instead of normal physiology of the vasa vasorum, is quite challenging and 
subject to the observer's interpretation. Although acceptable reproducibility of Gd+ was observed in the present study, a Gd+ quantitative measure would accurately increase Gdgrading. Density and thus enhancement of the vaso vasorum in normal extracranial arteries is poorly described in current literature, and between-patient and within-patient variability and its influence on wall enhancement is at this point unknown. In contrast to other vascular wall enhancement studies, the present study partly anticipated on this by also examining the contralateral artery as a reference. Of notice, only a minority (3/11) of patients with ipsilateral enhancement also had contralateral enhancement (one of whom had bilateral ECAA) so although not predictive of aneurysm growth, Gd-enhancement does seem to reflect disease activity of the vessel wall.

All three patients with ipsilateral brain infarcts showed ECAA wall-enhancement, but the majority of patients without infarcts also had aneurysm wall-enhancement. Brain infarcts and especially (small) cortical infarcts are often caused by thrombi-emboli from a proximal source such as the carotid arteries ${ }^{12}$. Silent brain infarcts have been associated with a three-fold increase in risk for stroke ${ }^{12}$. In asymptomatic patients with atherosclerotic stenosis of the carotid artery those with silent brain infarcts benefit more from revascularization procedures in terms of averting stroke risk compared to those without ${ }^{11}$. ECAA patients at risk for thromboembolic complications may also be identified by assessment of ipsilateral (silent) brain infarcts, in addition to assessment of ECAA wall Gd-enhancement or aneurysm growth. Structural imaging of the brain may contribute to clinical decision making on whether or not to treat (e.g. conservatively or surgically) these patients.

We found that two-third of patients had bilateral WMLs. As there was no clear asymmetry and presence of WML did not seem restricted to patients with ECAA wall-enhancement, based on our preliminary results WMLs do not seem directly related to Gd-enhancement of the aneurysm wall. Previous studies have shown that the cause of WML is multifactorial and thrombi-embolism from a proximal source is only one of the potential causes for these radiographic findings ${ }^{8,27}$. It is striking however that these relative young patients included in our study (median age 54) show considerably often a high (bilateral) WML score compared to literature investigating WMLs in an otherwise healthy population ${ }^{28}$. WMLs have been used widely as a surrogate marker for small vessel disease and have been closely associated with risk factors for atherosclerotic disease such as hypertension ${ }^{29}$. These findings suggest that not so much a causal relationship exists between the presence of ECAA and WMLs, but a shared etiology for presence of both diseases. WMLs have been associated with an increased risk of future stroke ${ }^{30}$ and cognitive decline ${ }^{29}$. Perhaps medical therapy in ECAA patients should be optimized to treat not only possible consequences of thrombi-emboli, but also to treat risk factors underlying generalized vascular disease. 


\section{Strengths \& Limitations}

The primary aim of our study was feasibility. Because of the small sample size, no conclusions can be drawn regarding the predictive value of wall-enhancement. Although good inter- and intra-observer reliabilities were found in our study, assessing presence of WMLs by means of the Fazekas scale can be highly dependent on expertise and experience. Also, the Fazekas scale is insensitive for subtle lesions and subtle changes over time. Other (semi-) quantitative measures, such as the rating scale for age-related white matter changes or computerized assessment of lesion volume, may be more reliable and sensitive to small changes over time. Future studies should include quantitative methods for determining white matter lesion volume so that subtle changes can be detected in an objective manner. Nevertheless, our method is easily translated to clinical practice and can straightforwardly be applied on frequently used MR sequences (FLAIR and T2). Little is known yet regarding the reliability of using MR for ECAA imaging compared to CT. Although high correlations between the two were found in studies on other type of aneurysms ${ }^{31}$, comparison of these two imaging techniques should be a topic for future research. The study sample is a reflection of clinical practice in ECAA disease, as a consequence various shapes and sizes of aneurysms with different blood flow characteristics were included. Slow blood flow within the aneurysm sac could cause 'pseudo-enhancement' which potentially affected the validity and clinical applicability of our results. Future computational hemodynamic and histological studies of aneurysm wall tissue should elucidate the relation of Gd-enhancement and aneurysm wall instability further. Lastly, one might argue that conventional diameter measurements fail to indicate ECAA growth. Since appropriate imaging tools to measure geometrical differences are currently lacking, we might have missed cases that expanded in other directions than obliquely.

\section{Conclusions}

In this small explorative study we demonstrated that extracranial carotid artery aneurysm wall imaging was feasible and Gd wall-enhancement was identified in the majority of aneurysms. Aneurysm growth was relatively common in a follow-up period of one year. Larger prospective studies are warranted to investigate the relation between ECAA wallenhancement and aneurysm growth, (silent) brain infarcts and white matter lesions. 


\section{References}

1. Pourier VEC, Welleweerd JC, Kappelle LJ, et al. Experience of a single center in the conservative approach of 20 consecutive cases of asymptomatic extracranial carotid artery aneurysms. Eur J Neurol. 2018;25(10):12851289.

2. Welleweerd JC, Moll FL, De Borst GJ. Technical options for the treatment of extracranial carotid aneurysms. Expert Rev Cardiovasc Ther. 2012;10(7):925-931.

3. Welleweerd JC, Den Ruijter HM, Nelissen BGL, et al. Management of Extracranial Carotid Artery Aneurysm. Eur J Vasc Endovasc Surg. 2015;50(2):141-147.

4. Welleweerd JC, Nelissen BGL, Koole D, et al. Histological analysis of extracranial carotid artery aneurysms. PLoS One. 2015;10(1):1-9.

5. Backes D, Hendrikse J, van der Schaaf I, et al. Determinants of Gadolinium-Enhancement of the Aneurysm Wall in Unruptured Intracranial Aneurysms. Neurosurgery. 2017;83(4):719-725.

6. Catherine Oppenheim, Naggara O, Touzé E, et al. High-Resolution MR Imaging of the Cer- vical Arterial Wall: What the Radiologist Needs to Know. RadioGraphics. 2009;29:1413-1431.

7. Jayasooriya G, Thapar A, Shalhoub J, et al. Silent cerebral events in asymptomatic carotid stenosis. J Vasc Surg. 2011;54(1):227-236.

8. Bouwhuijsen QJA Van Den, Vernooij MW, Verhaaren BFJ, et al. Carotid plaque morphology and ischemic vascular brain disease on MRI. Am J Neuroradiol. 2017;38(9):1776-1782.

9. Altaf N, Ed M, Morgan PS, et al. Hyperintensities Are Associated with Carotid Intraplaque Hemorrhage. Radiology. 2008;248(1).

10. Gensicke H, Van Der Worp HB, Nederkoorn PJ, et al. Ischemic brain lesions after carotid artery stenting increase future cerebrovascular risk. J Am Coll Cardiol. 2015;65(6):521-529.

11. Streifler JY, den Hartog AG, Pan S, et al. Ten-year risk of stroke in patients with previous cerebral infarction and the impact of carotid surgery in the Asymptomatic Carotid Surgery Trial. Int J Stroke. 2016;11(9):10201027.

12. Vermeer SE, Prins ND, den Heijer T, et al. Silent Brain Infarcts and the Risk of Dementia and Cognitive Decline. N Engl J Med. 2003;348(13):1215-1222.

13. Welleweerd JC, Bots ML, Kappelle LJ, et al. Rationale and design of the extracranial Carotid artery Aneurysm Registry (CAR). J Cardiovasc Surg (Torino). 2018;59(5):692-698.

14. Dieleman N, Yang W, Van Der Kolk AG, et al. Qualitative evaluation of a high-resolution 3D multi-sequence intracranial vessel wall protocol at 3 tesla MRI. PLoS One. 2016;11(8):1-11.

15. Yang H, Zhang X, Qin Q, et al. Improved cerebrospinal fluid suppression for intracranial vessel wall MRI. J Magn Reson Imaging. 2016;44(3):665-672.

16. de Leeuw F, de Groot J, Bots M, et al. Carotid atherosclerosis and cerebral white matter lesions in a population based magnetic resonance imaging study. J Neurol. 2000;247(4):291-296.

17. Fazekas F, Chawluk JB, Alavi A, et al. Mr Signal Abnormalities At 1.5-T in Alzheimer Dementia and Normal Aging. Am J Roentgenol. 1987;149(2):351-356.

18. Kim KW, MacFall JR, Payne ME. Classification of white matter lesions on magnetic resonance imaging in the elderly. Biol Psychiatry. 2008;64(4):273-280.

19. Kottner J, Audige L, Brorson S, et al. Guidelines for Reporting Reliability and Agreement Studies (GRRAS) were proposed. Int J Nurs Stud. 2011;48(6):661-671.

20. de Vet HCW, Terwee CB, Knol DL, et al. When to use agreement versus reliability measures. J Clin Epidemiol. 2006;59(10):1033-1039.

21. Aoki S, Aoki K, Ohsawa S, et al. Dynamic MR imaging of the carotid wall. J Magn Reson Imaging. 1999;9(3):420-427.

22. Qiao Y, Etesami M, Astor BC, et al. Carotid plaque neovascularization and hemorrhage detected by MR imaging are associated with recent cerebrovascular ischemic events. Am J Neuroradiol. 2012;33(4):755-760.

23. Kerwin WS, Brien KDO, Ferguson MS, et al. Inflammation in Carotid Atherosclerotic Plaque: A Dynamic Contrast-enhanced MR Imaging Study. Radiology. 2006;241(2):459-468.

24. Vergouwen MDI, Backes D, van der Schaaf IC, et al. Gadolinium Enhancement of the Aneurysm Wall in Unruptured Intracranial Aneurysms Is Associated with an Increased Risk of Aneurysm Instability: A FollowUp Study. Am J Neuroradiol. 2019:1-5. 
25. Krings T, Mandell DM, Kiehl TR, et al. Intracranial aneurysms: From vessel wall pathology to therapeutic approach. Nat Rev Neurol. 2011;7(10):547-559.

26. Portanova A, Hakakian N, Mikulis DJ, et al. Vasa vasorum 2013 insights and implications for imaging. Radiology. 2013;267(3):667-679.

27. Potter GM, Doubal FN, Jackson CA, et al. Lack of association of white matter lesions with ipsilateral carotid artery stenosis. Cerebrovasc Dis. 2012;33(4):378-384.

28. Leeuw F-E De, Groot JC De, Achten E, et al. Prevalence of cerebral white matter lesions in elderly people: A population based magnetic resonance imaging study. The Rotterdam Scan Study. J Neurol Neurosurg Psychiatry. 2001;70(1):9-14.

29. Van Dijk EJ, Prins ND, Vrooman HA, et al. Progression of cerebral small vessel disease in relation to risk factors and cognitive consequences: Rotterdam scan study. Stroke. 2008;39(10):2712-2719.

30. Streifler JY, Eliasziw M, Benavente OR, et al. Prognostic importance of leukoaraiosis in patients with symptomatic internal carotid artery stenosis. Stroke. 2002;33(6):1651-1655.

31. Acar T, Karakas AB, Ozer MA, et al. Building Three-Dimensional Intracranial Aneurysm Models from 3D-TOF MRA: a Validation Study. J Digit Imaging. 2019:963-970. 


\section{Supplemental Material}

Supplemental Table I. Patient characteristics

\begin{tabular}{lrrr}
\hline & $n=15$ & N/A \\
\hline Age at presentation, median in years (range) & 55 & $(40-69)$ & \\
\hline Men & 8 & $(53)$ & \\
\hline Medical history & & & 1 \\
$\quad$ Neurologic & 8 & $(57)$ & 2 \\
\hline Vascular & 6 & $(46)$ & 1 \\
\hline Trauma or surgery in cervical region & 4 & $(29)$ & 2 \\
\hline Cardiac & 1 & $(8)$ & 2
\end{tabular}

Family history

$\begin{array}{llll}\text { Cardiovascular } & 5 & (56) & 6 \\ \text { Aneurysm } & 2 & (25) & 7 \\ \text { history } & & & 5 \\ \text { No } & 7 & (70) \\ \text { Quit smoking } & 2 & (20) & \\ \text { Current smoker } & 1 & (10)\end{array}$

Medication use

2

$\begin{array}{lcc}\text { Anticoagulant } & 5 & (38) \\ \text { Lipid-lowering drugs } & 5 & (38)\end{array}$

\section{Presentation}

$\begin{array}{lll}\text { Coincidental finding } & 9 & (60) \\ \text { Referred by other specialist } & 5 & (33) \\ \text { Referred through GP } & 1 & (7)\end{array}$

Admission symptom

$\begin{array}{lcc}\text { Asymptomatic } & 11 & (73) \\ \text { Mass } & 2 & (13) \\ \text { Horner's syndrome } & 1 & (7) \\ \text { TIA in catchment area } & 1 & (7)\end{array}$

Presumed etiology

$\begin{array}{lcc}\text { Spontaneous, dissection } & 6 & (60) \\ \text { Trauma, dissection } & 2 & (20) \\ \text { Atherosclerosis } & 1 & (10) \\ \text { CTD } & 1 & (10)\end{array}$

Data are presented as number (\%) unless stated otherwise.

Abbreviations: $\mathrm{N} / \mathrm{A}=$ not available, $\mathrm{GP}=$ general practitioner, $\mathrm{TIA}=$ transient ischemic attack, $\mathrm{CTD}=$ connective tissue disorder. 

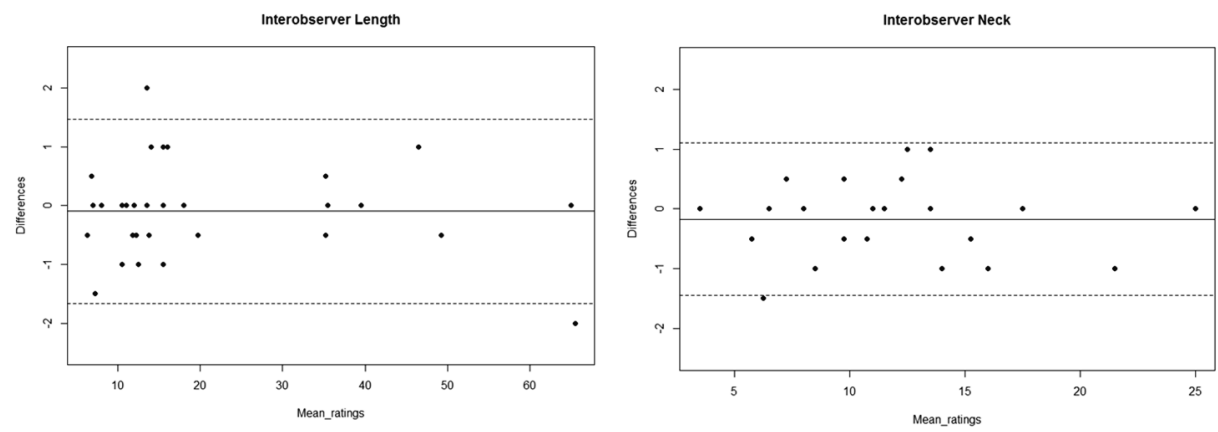

Supplemental Figure I \& II. Bland-Altman plots showing agreement of two observers on the maximum diameter measurement of 32 aneurysms ( 14 unilateral, 1 bilateral) for length and neck of the aneurysm sac. The line in the middle represents the mean difference of the volume between the two observers, and the dashed lines represent the upper and lower limits of agreement (mean difference $\pm 1.96 \times$ standard deviation).
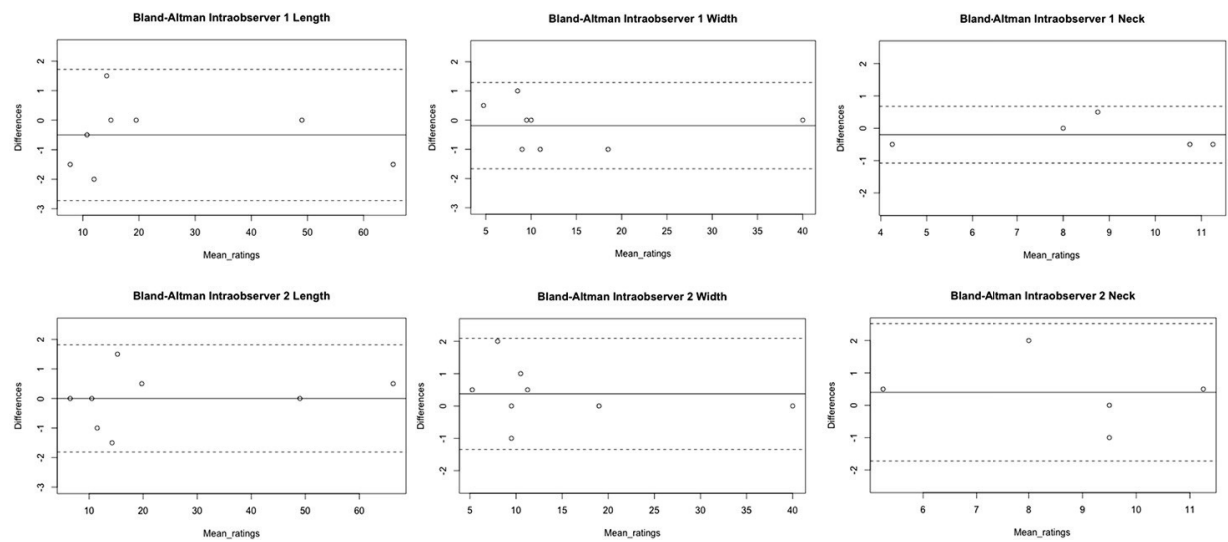

Supplemental Figure III-VIII. Bland-Altman plots showing intraobserver agreement on the maximum diameter measurement of eight unilateral aneurysms for length, width and neck of the aneurysm sac. The line in the middle represents the mean difference of the volume between the two observers, and the dashed lines represent the upper and lower limits of agreement (mean difference $\pm 1.96 \times$ standard deviation). 
Supplemental Table II. Inter- and intraobserver reliability and proportion of agreement

\begin{tabular}{|c|c|c|c|c|}
\hline Aneurysm size & \multicolumn{2}{|c|}{$\begin{array}{l}\text { Inter observer } \\
\text { ICC }(95 \% \mathrm{CI})\end{array}$} & \multicolumn{2}{|c|}{$\begin{array}{l}\text { Intra observer } \\
\text { ICC }(95 \% \mathrm{CI})\end{array}$} \\
\hline Length & \multicolumn{2}{|c|}{$0.999(0.998-0.999)$} & \multicolumn{2}{|c|}{$0.998(0.993-1.000)$} \\
\hline Width & \multicolumn{2}{|c|}{$0.995(0.980-0.998)$} & \multicolumn{2}{|c|}{$0.997(0.985-0.999)$} \\
\hline Neck & \multicolumn{2}{|c|}{$0.992(0.980-0.996)$} & \multicolumn{2}{|c|}{$0.965(0.827-0.998)$} \\
\hline Gadolinium uptake & Kappa (95\% CI $\left.{ }^{a}\right)$ & $\begin{array}{c}\text { Proportion of } \\
\text { agreement }\end{array}$ & Kappa (95\% CI a) & $\begin{array}{c}\text { Proportion of } \\
\text { agreement }\end{array}$ \\
\hline Ipsilateral & $0.913(0.698-1.000)$ & 0.960 & 1.000 & 1.000 \\
\hline Contralateral & $0.900(0.650-1.000)$ & 1.000 & 1.000 & 1.000 \\
\hline White matter lesions & $\begin{array}{l}\text { Weighted Kappa } \\
\qquad\left(95 \% \mathrm{CI}^{\mathrm{a}}\right)\end{array}$ & $\begin{array}{c}\text { Proportion of } \\
\text { agreement }\end{array}$ & $\begin{array}{l}\text { Weighted Kappa } \\
\left(95 \% \mathrm{CI}^{\mathrm{a}}\right)\end{array}$ & $\begin{array}{c}\text { Proportion of } \\
\text { agreement }\end{array}$ \\
\hline \multicolumn{5}{|l|}{ Periventricular } \\
\hline Ipsilateral & $0.911(0.757-0.979)$ & 0.828 & $0.818(0.231-1.000)$ & 0.625 \\
\hline Contralateral & $0.845(0.639-0.981)$ & 0.862 & $0.500(0.000-1.000)$ & 0.500 \\
\hline \multicolumn{5}{|l|}{ Deep } \\
\hline Ipsilateral & $0.899(0.690-1.000)$ & 0.900 & $0.946(0.250-1.000)$ & 0.875 \\
\hline Contralateral & $0.931(0.766-1.000)$ & 0.931 & 1.000 & 1.000 \\
\hline Infarction & Kappa $\left(95 \%\right.$ CI $\left.^{a}\right)$ & $\begin{array}{l}\text { Proportion of } \\
\text { agreement }\end{array}$ & Kappa $\left(95 \%\right.$ CI $\left.^{\mathrm{a}}\right)$ & $\begin{array}{c}\text { Proportion of } \\
\text { agreement }\end{array}$ \\
\hline \multicolumn{5}{|l|}{ Lacunar } \\
\hline Ipsilateral & 1.000 & 1.000 & $\mathrm{~N} / \mathrm{A}^{\mathrm{b}}$ & 0.875 \\
\hline Contralateral & 1.000 & 1.000 & $\mathrm{~N} / \mathrm{A}^{\mathrm{b}}$ & 0.875 \\
\hline \multicolumn{5}{|l|}{ Subcortical } \\
\hline Ipsilateral & \multicolumn{2}{|c|}{0 observations } & \multicolumn{2}{|c|}{0 observations } \\
\hline Contralateral & \multicolumn{2}{|c|}{0 observations } & \multicolumn{2}{|c|}{0 observations } \\
\hline \multicolumn{5}{|l|}{ Cortical } \\
\hline Ipsilateral & 1.000 & 1.000 & \multicolumn{2}{|c|}{0 observations } \\
\hline Contralateral & 1.000 & 1.000 & 1.000 & 1.000 \\
\hline
\end{tabular}

Abbreviations: $\mathrm{ICC}=$ intraclass correlation coefficient, $\mathrm{CI}=$ confidence interval, $\mathrm{N} / \mathrm{A}=$ not available. ${ }^{\mathrm{a}} 95 \% \mathrm{CI}$ for (weighted) kappa were obtained by bootstrapping with $n=1000 .{ }^{b}$ Not available, since only one observation was $\neq 0$. 



\title{
Chapter 9
}

\section{Carotid tortuosity is associated with the presence of an extracranial carotid artery aneurysm}

\author{
Submitted
}

C.J.H.C.M. van Laarhoven

J. Klaassen

E.E. de Vries

Q.M.J. van der Vliet

C.E.V.B. Hazenberg

M.L. Bots

G.J. de Borst 


\section{Abstract}

\section{Background and purpose}

Tortuous arteries may be associated with cervical dissection, and this intima disruption of the carotid artery is a common cause of extracranial carotid artery aneurysms (ECAA). In the present study we aimed to investigate if carotid tortuosity is associated with ECAA, in patients without presence or history of carotid artery dissection.

\section{Methods}

A cross-sectional case-control study was performed including 35 unilateral ECAA patients (cases) and 105 age- and sex-matched controls, all without presence or history of carotid dissection. Tortuosity was expressed as tortuosity-index (TI), curvature, and torsion measured on CTA data in 3Mensio Vascular and MATLAB by two independent operators. Primary comparison was tortuosity in ipsi- versus contralateral carotid artery within the ECAA patient, and secondary comparison was with ipsilateral carotid arteries in control patients. All observations were assessed on inter- and intraoperator reproducibility.

\section{Results}

Carotid tortuosity was comparable within the ECAA patient (Spearman correlation 0.758, $p<0.001$ ), yet distinctively higher in comparison with unilateral controls. After adjustment for patient characteristics, presence of ECAA ( $\beta 0.146,95 \%$ CI 0.100-0.192) was associated with TI. All tortuosity observations showed excellent inter- and intraoperator reproducibility.

\section{Conclusion}

This cross-sectional case-control analysis reveals that carotid tortuosity is similar in both carotids within ECAA patients, though significantly higher in comparison with age- and sex-matched controls. 


\section{Introduction}

The extracranial carotid artery aneurysm (ECAA) is a rare vascular disease, which clinical course remains not fully understood ${ }^{1}$. The clinical presentation is highly dependent on the etiology, carotid site, and size of the carotid aneurysm ${ }^{2}$. Registry data from the ongoing international web-based Carotid Aneurysm Registry ${ }^{3}$ (CAR; www. carotidaneurysmregistry.com) indicate that approximately half of patients presents with local symptoms (e.g. pulsatile mass), of which one sixth is affected by cerebral ischemia (stroke or transient ischemic attack). It has been suggested that arterial tortuosity in the course of the carotid artery such as sharp bends and kinks may affect the hemodynamics, resulting in either dilated or stenotic lesions as a consequence ${ }^{4-6}$. Moreover, tortuosity of the cervical arteries (vertebral and carotid) has been suggested to be associated with arterial dissection, and this intima disruption of the carotid artery at its turn is a common cause of ECAA ${ }^{1,7-9}$. Earlier research geometrically differentiated between carotid loops, S- and C-bends $s^{710-13}$, which were interpreted from angiographies from different modalities. As 2D images for evaluation of geometry are highly subjective to visual projection of the angiography (e.g. derived from DSA $)^{11,14,15}$, more reliable quantitative estimates indicating severity of tortuosity can be obtained by $3 \mathrm{D}$ reconstruction, such as tortuosity index (TI), curvature, and torsion ${ }^{8,9,16-19}$. TI is the ratio of the length of curved, or central luminal line (CLL) and the straight line length between two anatomical landmarks ${ }^{9,16,17,20}$. Besides differences in length, curvature and torsion values may further indicate remarkable geometry ${ }^{5,21,22}$. Curvature is defined as the degree a line deviates from being straight, and torsion expresses the direction and degree of turns of a line $e^{21}$. If carotid tortuosity is associated with carotid aneurysms without arterial dissection, this may be integrated in a screening algorithm for ECAA.

The objective of this cross-sectional case-control study was to investigate if a high degree of carotid tortuosity is associated with ECAAs, in patients without presence or history of carotid dissection. Carotid tortuosity was compared within cases, and between cases and age- and sex-matched controls.

\section{Methods}

\section{Participants}

The article was written in accordance with the STROBE ${ }^{23}$ statements. Local ethical approval for this study was obtained, and all research was conducted according to the principles of the Declaration of Helsinki (59th amendment, Seoul 2008) and in accordance with the Dutch Medical Research Involving Human Subjects Act (WMO). A crosssectional case-control study was conducted from single-center data from the University Medical Center ${ }^{\star}$ blinded $^{\star}$ included in the CAR (www.carotidaneurysmregistry.com). 
The registry protocol has been published previously ${ }^{3}$. In short, any patient aged 18 -years or older diagnosed with an ECAA is included in this ongoing registry, independent of etiology or treatment strategy. Baseline characteristics and imaging follow-up data were collected in a prospective manner. All patients provided informed consent. For the present study, a case was defined as a diagnosed ECAA patient included in the CAR. Cases were equally selected from the registry based on sex, age (younger and $\geq 55$ years old), shape of aneurysm (fusiform or saccular), and both small (maximum diameter $\leq 10.0 \mathrm{~mm})$ and large $(>10.0 \mathrm{~mm})$ ECAAs. The primary thin-slice $(<1.0 \mathrm{~mm})$ CTA carotids to confirm the ECAA diagnosis was used for this study. Age- and sex-matched controls were enrolled from subjects whom underwent a thin-slice CTA carotids as part of a trauma screening in the UMCU during the same study period. Cases and controls were matched in a ratio 1:3, with similar sex and age (range of 1 year). Both cases and controls were excluded and replaced if the arterial phase of the CTA carotids was poor. Additionally, cases were excluded in case of presence or history of carotid arterial dissection, mycotic, iatrogenic, dissecting, or bilateral ECAA. Controls were excluded in case of a trauma with direct impact on the neck region and/or diagnosis of traumatic cervical artery dissection.

\section{Clinical data}

Vascular risk factors, including hypertension, diabetes mellitus, smoking, connective tissue disease (CTD), rheumatoid arthritis, and statin use of the cases were obtained from the CAR database. ECAA was defined as $\geq 150 \%$ fusiform dilatation of the carotid artery in comparison with the contralateral side, and saccular aneurysms of any size were accepted $^{24}$. Similar patient characteristics of the controls were obtained by patient chart review. The following definitions were used throughout the study: hypertension was defined as the use of any blood pressure lowering medication, or blood pressure $>140 / 90$ $\mathrm{mmHg}$ on repeated measurement. Diabetes was defined as usage of any blood glucose lowering medication. Current smoking was considered as tobacco usage within last six months. CTD was defined as any genetically proven disorder, e.g. Marfan, Loeys-Dietz, Ehlers Danlos syndrome, and rheumatoid arthritis was scored if diagnosed by a rheumatologist. Any prescribed statin was scored as statin use.

\section{Imaging protocol}

All CTA studies were performed using a 64- or 128-slice CT scanner (Philips Brilliance; Philips Medical Systems, Best, The Netherlands). Median slice thickness was $0.67 \mathrm{~mm}$ (range $0.62-0.90 \mathrm{~mm}$ ), increment 0.33 , collimation $64 \mathrm{x} 0.625$ and pitch 1.2 . Radiation exposure parameters were 100-120 kilovolt and 150-300 milliampere second. The field of view was set per patient. Injection of 65 milliliter intravascular contrast (Lopromide, Schering, Berlin, Germany) was followed by a saline bolus of 40 milliliter, both at a flow rate of 6.0 milliliter per second. 


\section{Imaging analysis}

Every CTA scan was analysed according to a pre-defined protocol with software of 3mensio Vascular (version 9.2, Pie Medical Imaging B.V., Maastricht, the Netherlands). Using the vessel tool, a 3D image of the carotid arteries was generated. The central luminal line (CLL) of the carotid artery was automatically created and manually corrected. The carotid arteries were segmented in the curved planar reformation (CPR) of the vessel, measured from skull base to carotid bifurcation (internal carotid artery; ICA), and to the origin of the common carotid artery (CCA). The origin of the CCA was defined as the first slice in perpendicular plane in which the CCA was visible. The CLL length of the total carotid artery was calculated by adding lengths of ICA and CCA (Figure 1). The TI was measured on the CPR image as a ratio of the CLL length of the vessel to the straight line length between the two endpoints:

$$
T I=\frac{(\text { CLL length) }}{\text { (Straight line length) }}
$$

Additionally, coordinates of the $1.0 \mathrm{~mm}$ interpolated CLL were exported from 3 mensio Vascular, and analysed with an in-house MATLAB (vR2019b, MathWorks', Natick, Massachusetts, USA) based script to calculate the curvature and torsion of the total carotid artery. Applied equations for calculation of the extrinsic linear curvature and torsion are described in Supplemental Material I. Based on literature, the following cutoff values were applied; low $(\leq 0.15)$, medium $(>0.15$ and $\leq 0.3)$, high $(>0.3)$ curvature, and low $(\leq 5.0)$, and high $(>5.0)$ torsion values across the CLL of the entire carotid artery from CCA origin up to skull base. Total number of curvature and torsion categories per study subject were reported (Figure 2). All measurements were obtained by two independent operators, of which one was blinded for outcome. Case or control status of the study subjects was blinded for both operators while measuring carotid tortuosity. To assess intraoperator variability, 35 scans (20\%) were randomly selected and measured twice with a minimum wash-out period of two weeks. Both the operators were trained with five test patients, and the sequence of study patient measurements was determined by randomization to overcome a potential effect from learning.

\section{Sample size calculation}

The literature was reviewed for previous TI measurements of the extracranial carotid arteries for an accurate sample size calculation ${ }^{8,9,16,19,20}$. For the healthy carotid artery, a mean TI of 1.2 was used, for the ECAA-affected carotid artery mean TI of 1.35, with standard deviation of 0.25 . As a result, we obtained a sample size of 35 cases and 105 1:3 matched controls, 140 patients in total, one-sided with a power of $80 \%$ and type I error of $5 \%$, calculated by use of the package ' $e p i R^{\prime 25}$. 


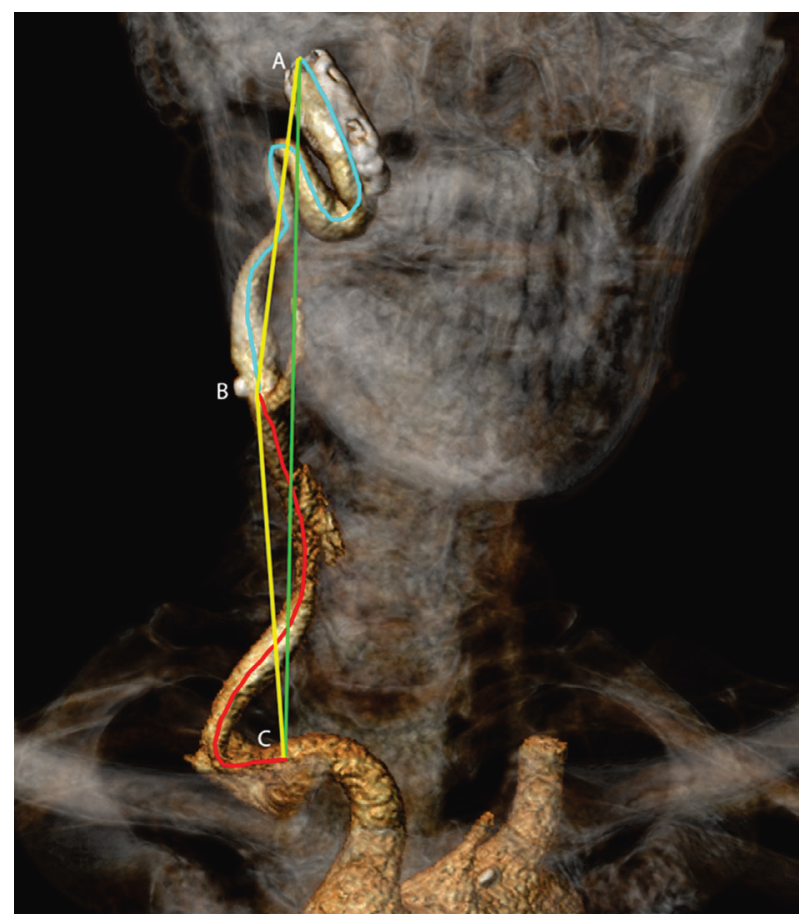

Figure 1. Segmentation example of $69 y$-o male with a extracranial carotid aneurysm of the right internal carotid artery (ICA). The carotid tortuosity index was calculated for every study patient as ratio of the length of the central luminal line (CLL) and the straight line length. The carotid artery was measured from skull base (A) until carotid bifurcation (B), and origin of the CCA (C). The CLL for the ICA is indicated in blue, for the common carotid artery (CCA) in red. The CLL of the total carotid artery was calculated by adding ICA and CCA CLL length. The straight lines are indicated in yellow and green.

\section{Outcome and statistical analysis}

The primary parameter of interest was the difference of TI of the affected ipsilateral ECAA carotid artery compared with the contralateral artery within one ECAA patient, in order to correct for all confounding factors. The secondary parameter of interest was defined as the difference in TI in the affected carotid artery of ECAA patients compared with ipsilateral control carotids, adjusted for potential confounding factors. Additionally, number of curvature and torsion values of the carotid artery were analysed in these groups. All observations were assessed in terms of inter- and intraoperator reliability and agreement. Reliability was assessed by use of Intraclass correlation coefficient (ICC; model: two-way mixed, type: absolute agreement) with 95\% confidence intervals (CI). Bland-Altman analysis was used to assess agreement for $\mathrm{TI}^{26,27}$. Non-parametrically distributed continuous and categorical baseline differences were compared with Mann Whitney $\mathrm{U}$ test, and $\chi$ z test respectively. 
A
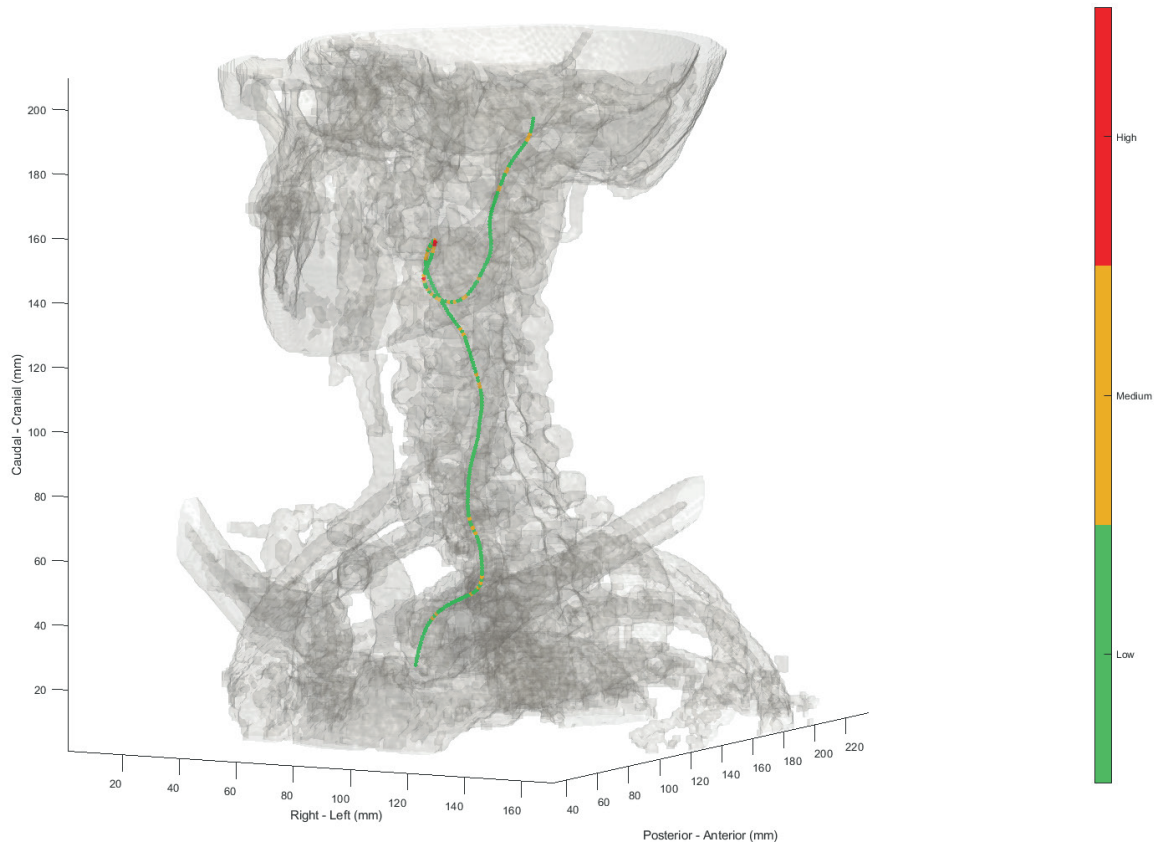

\section{B}

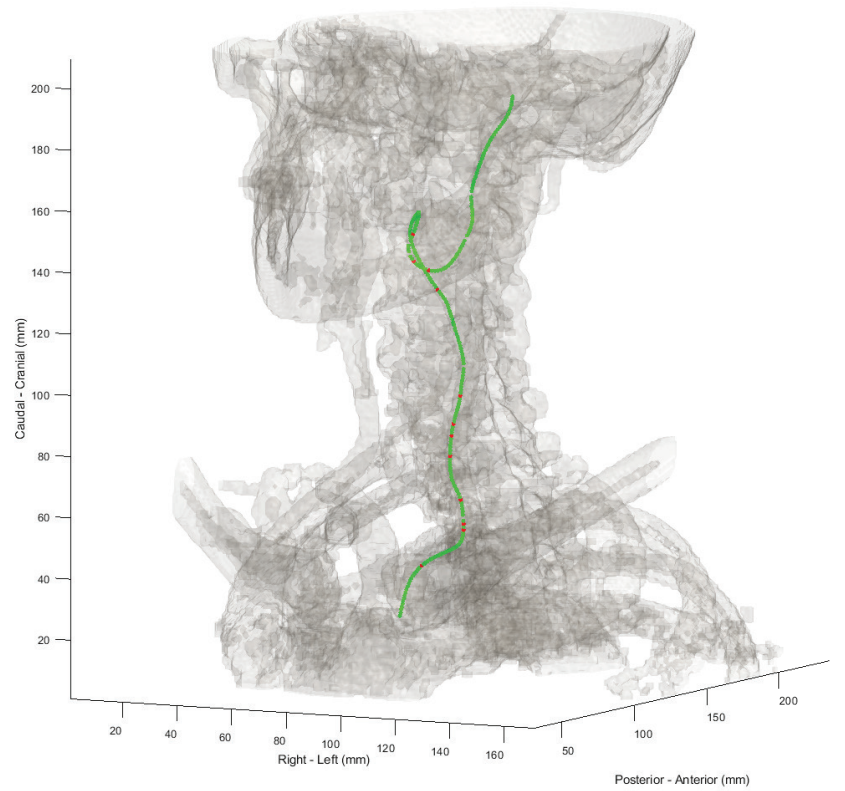

Figure 2. Example of curvature and torsion measures of the left carotid artery of a $62 \mathrm{y}-\mathrm{o}$ female. The colored line represents the central luminal line of the total carotid artery (CA), $A$ : curvature across the total CA divided in low (green; $\leq 0.15$ ), medium (orange; $>0.15$ and $\leq 0.30$ ), and high curvature (red; $>0.30$ ), $B$ : torsion divided in low torsion (green; $\leq 5.0$ ), and high torsion $($ red; $>0.5)$. Applied equations are summarized in Supplemental Material I. 


\section{Carotid tortuosity within the ECAA patient}

Continuous measures within one ECAA patient were compared by use of the paired T-test (parametrical), or Wilcoxon signed rank test (non-parametrical data). The correlation between continuous values was assessed with Pearson (parametrical) or Spearman correlation (non-parametrical data).

\section{Carotid tortuosity compared in ECAA patients and controls}

Cases and controls were compared by use of the independent T-test, or Mann-Whitney U-test. Multiple linear regression analysis was used to correct for confounding. Potential confounders were selected based on a univariate analysis $(p<0.1)$ and literature $e^{5,7,10,14,20,28}$. Regression coefficients with $95 \% \mathrm{CI}$ were reported, and $P$-values $<0.05$ were considered statistically significant. All statistical analyses were conducted using SPSS v25.0 (IBM SPSS Statistics for Windows, Version 25.0. Armonk, NY: IBM Corp.) and Rstudio v3.4.1 (RStudio Team (2016). RStudio: Integrated Development for R. RStudio, Inc., Boston, MA, www.rstudio.com).

\section{Results}

From the CAR database, thirty-five unilateral ECAA cases were selected based on sex, age, shape and size of the ECAA. Out of $>3,000$ trauma patients, 105 eligible control patients were included. Baseline characteristics of the separate patient groups are summarized in Table I. Half of the patients (51\%) were men with a median age of 62 years-old (range 25-82). No differences in cases and controls were observed in terms of cardiovascular medical history. Reported maximum diameters of ECAAs were median $13.5 \mathrm{~mm}$ (range 4.5-40.5).

\section{Inter- and intraoperator reproducibility}

In total, 210 TI measurements were performed in 140 unique study subjects. Excellent inter- and intraoperator reliability was observed with ICCs $\geq 0.9$ (Supplemental Table I). Bland-Altman plots showed no systematic bias and limits of agreement were exceeded by less than $10 \%$ of the total dataset, indicating normal differences (Supplemental Figure I and II). There were no significant differences in TI observed in blinded and non-blinded operators (data not shown).

\section{Carotid tortuosity within the ECAA patient}

Measures of TI, curvature and torsion of the different subfields of the carotid arteries of included ECAA patients are shown in Table 2. No distinctive differences in the unilateral ECAA artery and contralateral non-affected extracranial carotid artery in any of the 
Table 1. Baseline characteristics of study population.

\begin{tabular}{lccccc}
\hline & $\begin{array}{c}\text { ECAA patients } \\
\mathbf{n}=\mathbf{3 5}^{*}\end{array}$ & $\begin{array}{c}\text { Control patients } \\
\mathbf{n}=\mathbf{1 0 5}\end{array}$ & $\boldsymbol{p}$-value \\
\hline Male & $\mathrm{n}$ & $\%$ & $\mathrm{n}$ & $\%$ & \\
\hline Age at scan, median in years (range) & 18 & 51 & 54 & 51 & 1 \\
\hline Carotid side & 62 & $(25-82)$ & 62 & $(25-82)$ & 0.983 \\
\hline \multicolumn{1}{c}{ Right } & 18 & 51 & & & 1 \\
\hline Hypertension & 16 & 47 & 54 & 51 & \\
\hline Rheumatoid arthritis & 3 & 9 & 34 & 33 & 0.179 \\
\hline Diabetes mellitus & 3 & 9 & 6 & 6 & 0.688 \\
\hline Connective tissue disorder & 2 & 6 & 7 & 7 & 0.706 \\
\hline Statin use & 14 & 41 & 0 & 0 & 0.058 \\
\hline
\end{tabular}

Abbreviations: ECAA = extracranial carotid artery aneurysm, ${ }^{\star}$ : for 1 case no medical history was available.

Table 2. Tortuosity measures in cases, compared with own contralateral carotid artery.

\begin{tabular}{|c|c|c|c|c|c|c|}
\hline & & \multicolumn{2}{|c|}{$\begin{array}{l}\text { Ipsilateral ECAA carotid artery } \\
\qquad \mathbf{n}=35\end{array}$} & \multicolumn{2}{|c|}{$\begin{array}{l}\text { Contralateral carotid artery } \\
\qquad \mathbf{n}=\mathbf{3 4}^{*}\end{array}$} & $p$-value \\
\hline \multirow[t]{3}{*}{ ICA } & & 1.387 & $(1.066-2.145)$ & 1.346 & $(1.022-2.028)$ & 0.369 \\
\hline & Left carotid & 1.398 & $(1.072-1.840)$ & 1.357 & $(1.022-1.681)$ & 0.163 \\
\hline & Right carotid & 1.335 & $(1.066-2.145)$ & 1.287 & $(1.026-2.028)$ & 0.795 \\
\hline \multirow[t]{3}{*}{ CCA } & & 1.108 & $(1.016-1.694)$ & 1.089 & $(1.010-1.864)$ & 0.573 \\
\hline & Left carotid & 1.108 & $(1.021-1.424)$ & 1.084 & $(1.014-1.406)$ & 0.055 \\
\hline & Right carotid & 1.105 & $(1.016-1.694)$ & 1.143 & $(1.010-1.864)$ & 0.344 \\
\hline \multirow[t]{3}{*}{ Total CA } & & 1.245 & $(1.046-1.793)$ & 1.229 & $(1.023-1.943)$ & 0.726 \\
\hline & Left carotid & 1.249 & $(1.046-1.553)$ & 1.227 & $(1.023-1.491)$ & 0.381 \\
\hline & Right carotid & 1.241 & $(1.080-1.793)$ & 1.233 & $(1.028-1.943)$ & 0.586 \\
\hline \multicolumn{7}{|c|}{ Curvature (counts) ${ }^{* *}$} \\
\hline Low & $\leq 0.15$ & 186 & $(147-230)$ & 195 & $(148-240)$ & 0.565 \\
\hline Medium & $0.16-0.30$ & 40 & $(23-60)$ & 39 & $(5-360)$ & 0.280 \\
\hline High & $>0.30$ & 5 & $(0-15)$ & 5 & $(0-20)$ & 0.312 \\
\hline \multicolumn{7}{|c|}{ Torsion (counts) ${ }^{\star *}$} \\
\hline Low & $\leq 5.0$ & 227 & $(177-525)$ & 229 & $(177-259)$ & 0.627 \\
\hline High & $>5.0$ & 7 & $(1-13)$ & 7 & $(1-16)$ & 0.599 \\
\hline
\end{tabular}

All values are presented as median (range). Abbreviations: $\mathrm{ECAA}=$ extracranial carotid artery aneurysm, ICA $=$ internal carotid artery, $\mathrm{CCA}=$ common carotid artery, $\mathrm{CA}=$ carotid artery. ${ }^{*}$ One patient with contralateral occlusion, ${ }^{*}$ Only 33 vs 30 segmentations available for curvature and torsion calculation across total CA. 
subfields (ICA, CCA, or total CA) were seen. The highest TIs were observed in the ICA throughout. Medium and high curvature numbers were rare across both carotids, indicating similar counts of sharp bends in both carotids. Lastly, nearly similar numbers of torsion were observed. Spearman correlation between the ipsi- and contralateral TI of the total carotid artery was $0.758(p<0.001)$.

\section{Carotid tortuosity compared in ECAA patients and controls}

Significant differences in TI were observed when comparing the ECAA affected extracranial carotid artery with unilateral controls (Table 3). Also, TI was higher in the ICA than CCA or total carotid artery. Both medium and high curvature counts across the total CA were higher within cases compared to controls $(p<0.000)$. In contrast, for high torsion counts, no differences were observed.

Multiple linear regression was performed on TI measures of total carotid artery in unilateral cases and controls. Covariates were age $\geq 65$ years-old, sex, carotid side, hypertension, CTD, and lastly presence of ECAA. Presence of ECAA ( $\beta$ 0.146, 95\% CI $0.100-0.192, p<0.001)$ was independently associated with an increased TI.

Table 3. Tortuosity measures of cases and sex- and age-matched controls.

\begin{tabular}{|c|c|c|c|c|c|c|}
\hline & & \multicolumn{2}{|c|}{$\begin{array}{l}\text { Ipsilateral ECAA carotid artery } \\
\qquad \mathbf{n}=\mathbf{3 5}\end{array}$} & \multicolumn{2}{|c|}{$\begin{array}{l}\text { Control carotid artery } \\
\qquad n=105\end{array}$} & \multirow[t]{2}{*}{ p-value } \\
\hline \multicolumn{6}{|l|}{ Tortuosity index } & \\
\hline \multirow[t]{3}{*}{ ICA } & & 1.387 & $(1.066-2.145)$ & 1.122 & $(1.009-2.048)$ & $<0.001$ \\
\hline & Left carotid & 1.398 & $(1.072-1.840)$ & 1.150 & $(1.017-2.048)$ & $<0.001$ \\
\hline & Right carotid & 1.335 & $(1.066-2.145)$ & 1.103 & $(1.018-1.501)$ & $<0.001$ \\
\hline \multirow[t]{3}{*}{ CCA } & & 1.108 & $(1.016-1.694)$ & 1.051 & $(1.008-1.373)$ & 0.010 \\
\hline & Left carotid & 1.108 & $(1.021-1.424)$ & 1.050 & $(1.008-1.287)$ & 0.029 \\
\hline & Right carotid & 1.105 & $(1.016-1.694)$ & 1.056 & $(1.010-1.373)$ & 0.182 \\
\hline \multirow[t]{3}{*}{ Total CA } & & 1.245 & (1.046-1.793) & 1.105 & $(1.018-1.570)$ & $<0.001$ \\
\hline & Left carotid & 1.249 & $(1.046-1.553)$ & 1.109 & $(1.021-1.570)$ & $<0.001$ \\
\hline & Right carotid & 1.241 & $(1.080-1.793)$ & 1.103 & $(1.018-1.501)$ & $<0.001$ \\
\hline \multicolumn{7}{|c|}{ Curvature (counts)* } \\
\hline Low & $\leq 0.15$ & 186 & $(147-230)$ & 189 & $(139-234)$ & 0.716 \\
\hline Medium & $0.16-0.30$ & 40 & $(23-60)$ & 24 & $(2-52)$ & $<0.001$ \\
\hline High & $>0.30$ & 5 & $(0-15)$ & 0 & $(0-11)$ & $<0.001$ \\
\hline \multicolumn{7}{|c|}{ Torsion (counts) ${ }^{*}$} \\
\hline Low & $\leq 5.0$ & 227 & $(177-525)$ & 200 & $(159-252)$ & $<0.001$ \\
\hline High & $>5.0$ & 7 & $(1-13)$ & 6 & $(2-12)$ & 0.203 \\
\hline
\end{tabular}

All values are presented as median (range). Bold indicates $p<0.05$. Abbreviations: ECAA $=$ extracranial carotid artery aneurysm, $\mathrm{ICA}=$ internal carotid artery, $\mathrm{CCA}=$ common carotid artery, $\mathrm{CA}=$ carotid artery. ${ }^{*}$ Only 33 vs 92 segmentations available for curvature and torsion calculation across total CA. 


\section{Discussion}

The present cross-sectional case-control study showed that carotid tortuosity is similar in the affected ipsi- versus non-affected contralateral carotid artery within ECAA patients, but distinctively higher in comparison with control patients. The difference between cases and controls remained after adjustment for potential confounding factors. Both excellent inter- and intraoperator reproducibility was observed in all TI measurements.

Both $\mathrm{TI}^{9,14}$ and curvature ${ }^{5,14,29}$ of the carotids were investigated in patients with intracranial aneurysms, and even though the methods used to assess tortuosity varied, associations of high tortuosity with aneurysm formation were reported. The finding that arterial bends affect hemodynamic forces, was confirmed by several computational hemodynamic studies $^{5,29,30}$. Fluctuating wall shear stresses caused slow flow within aneurysms, and areas with highest curvature were prone to aneurysm formation ${ }^{4,29,30}$. Though, the question remains which pathophysiological process or processes initiated vascular remodeling, and with that dynamic hemodynamic forces. Identification of these initiating factors could elucidate the core of aneurysmal disease, and may point out new targets for therapeutic options.

The present study confirms the association of high arterial tortuosity and aneurysm presence for the extracranial carotid artery. Available literature reports most ECAAs to be located within the ICA ${ }^{31-33}$, and in this subfield the highest values of TI were measured (Table 2,3). Since arterial tortuosity is high in ECAA patients, this may hamper endovascular treatment options due to hostile landing zones of the stent graft. At this point, optimal treatment and follow-up of ECAAs is largely unknown ${ }^{2,34}$. Invasive endovascular or surgical treatment seems to be indicated in ECAA patients with symptoms and/or growing aneurysms ${ }^{34}$. The effect of tortuosity on endovascular stenting was recently studied in thoracic aneurysm patients. By use of computational hemodynamics studies, impressive displacement forces after TEVAR were observed in patients with thoracic tortuosity ${ }^{35}$. Even though the blood pressure is likely to be lower within the carotids, the tortuous anatomy could mimic displacement forces after carotid stenting and cause migration of the stent. Defining a quantitative tortuosity threshold for endovascular therapy, will aid in selecting eligible patients for endovascular stenting of ECAA. Moreover, future computational hemodynamics studies in ECAA patients could also enhance optimizing stent graft design for tortuous carotids. Although our study was powered on the difference in TI for case and control patients, we have additionally analyzed the ipsi- and contralateral artery within ECAA patients in contrast to other studies ${ }^{5,11,14}$. All visible and invisible confounding factors for tortuosity were corrected in this analysis. The affected ECAA carotid artery showed overall higher TI 
values than the contralateral side, but somewhat surprisingly no significant differences were observed, nor in curvature or torsion values (Table 2). Similar bilateral TI was also observed in a sample of extracranial artery dissection patients ${ }^{9}$, though only a small number developed multiple dissections. Bilateral tortuosity may indicate that these patients have a generalized tortuous vascular subtype, although it remains unclear if this predisposes for bilateral ECAA disease. Future longitudinal research is warranted to overcome antecedent-consequent bias, and further elucidate the etiology of aneurysmal and tortuous carotids. The extensiveness of tortuosity and aneurysmal disease in patients with ECAA could be assessed additionally ${ }^{33}$.Ideally, arterial tortuosity could be used as surrogate marker of a vascular subtype prone to aneurysm formation. Further, it is imaginable that ECAAs with large diameters affect the carotid geometry more, and growth of the aneurysm sac could potentially alter future endovascular options. As the present study was not powered on the difference in tortuosity for aneurysm size, stratification into large $(>10 \mathrm{~mm})$ and small $(\leq 10 \mathrm{~mm})$ ECAA did not influence the results within the ECAA patient ( $p>0.338$, data not shown). Our study results can be used as baseline values for future research to investigate the difference in arterial tortuosity stratified in ECAA size. Besides age $\mathrm{e}^{7,20,28}$ and aneurysm presence $\mathrm{e}^{5,14,15}$, male sex, hypertension ${ }^{10}$, and CTDs ${ }^{13}$ were suggested to be associated with tortuosity. In the present analysis, we confirm these findings for age and aneurysm presence.

\section{Limitations}

Some limitations need to be taken into account while interpreting the present results. Although we opted to exclude both case and control patients with presence or history of carotid dissection, selection of a dissection-free study sample is challenging. Intima ruptures may be asymptomatic, and radiological identification of the characteristic intima flap or mural hematoma is hampered by time-dependent vascular remodeling. The potential influence of dissection on carotid tortuosity and ECAA, is therefore not completely ruled out. Secondly, assessment of curvature and torsion is rarely investigated for the extracranial carotid arteries. In order to report our results accessibly, we arbitrarily defined cut-off values to indicate degree of curvature and torsion (Figure 2). Suitable thresholds and their clinical applicability remain to be investigated. In addition, curvature and torsion values were solely available for the total CLL and thus entire carotid artery. Further optimization of software should be performed to discriminate and assess curvature and torsion in the ICA and CCA separately. As in every medical imaging derived study, the present results are highly dependent on quality of the scan. Deviating head- and neck-postures while scanning could influence carotid geometry, in particular the length of the straight line of the carotid artery. Since we observed angulation in only a few subjects, both cases and controls, we consider this influence neglectable. In addition, despite manual correction of the automatically generated CLL by the 3 mensio Vascular 
software was necessary in every study subject causing potential variability of study results, reproducibility of the present tortuosity measures between operators was excellent. Quantitative 3D tortuosity measures are therefore recommended in future tortuosity research.

\section{Conclusions}

This cross-sectional case-control study showed that carotid tortuosity is similar in both carotids within ECAA patients, though significantly higher in comparison with age- and sex-matched controls. 


\section{References}

1. Welleweerd JC, Nelissen BGL, Koole D, et al. Histological analysis of extracranial carotid artery aneurysms. PLoS One 2015;10:1-9.

2. Welleweerd JC, Den Ruijter HM, Nelissen BGL, et al. Management of Extracranial Carotid Artery Aneurysm. Eur J Vasc Endovasc Surg 2015;50:141-7.

3. Welleweerd JC, Bots ML, Kappelle LJ, et al. Rationale and design of the extracranial Carotid artery Aneurysm Registry (CAR). J Cardiovasc Surg (Torino) 2018;59:692-8.

4. Dolan JM, Kolega J, Meng H. High wall shear stress and spatial gradients in vascular pathology: A review. Ann Biomed Eng 2013;41:1411-27.

5. Lauric A, Safain MG, Hippelheuser J, et al. High curvature of the internal carotid artery is associated with the presence of intracranial aneurysms. J Neurointerv Surg 2014;6:733-9.

6. Norman PE, Powell JT. Site specificity of aneurysmal disease. Circulation 2010;121:560-8.

7. Saba L, Argiolas GM, Sumer S, et al. Association between internal carotid artery dissection and arterial tortuosity. Neuroradiology 2015;57:149-53.

8. Giossi A, Mardighian D, Caria F, et al. Arterial tortuosity in patients with spontaneous cervical artery dissection. Neuroradiology 2017;59:571-5.

9. Kim BJ, Yang E, Kim NY, et al. Vascular Tortuosity May Be Associated with Cervical Artery Dissection. Stroke 2016;47:2548-52.

10. Zenteno M, Viñuela F, Moscote-Salazar LR, et al. Clinical implications of internal carotid artery tortuosity, kinking and coiling: a systematic review. Rom Neurosurg 2014;21:51-60.

11. Labeyrie PE, Braud F, Gakuba C, et al. Cervical artery tortuosity is associated with intracranial aneurysm. Int J Stroke 2017;12:549-52.

12. Dittrich R, Nassenstein I, Harms S, et al. Arterial elongation ("redundancy") is not a feature of spontaneous cervical artery dissection. J Neurol 2011;258:250-4.

13. Welby JP, Kim ST, Carr CM, et al. Carotid artery tortuosity is associated with connective tissue diseases. Am J Neuroradiol 2019;40:1738-43.

14. Kliś K, Krzyżewski R, Kwinta B, et al. Tortuosity of the Internal Carotid Artery and Its Clinical Significance in the Development of Aneurysms. J Clin Med 2019;8:237.

15. Kim BJ, Lee SH, Kwun BD, et al. Intracranial Aneurysm Is Associated with High Intracranial Artery Tortuosity. World Neurosurg 2018;112:e876-80.

16. de Vries EE, Pourier VEC, van Laarhoven CJHCM, et al. Comparability of semiautomatic tortuosity measurements in the carotid artery. Neuroradiology 2019;61:147-53.

17. Chen CK, Chou HP, Guo CY, et al. Interobserver and intraobserver variability in measuring the tortuosity of the thoracic aorta on computed tomography. J Vasc Surg 2018;68:1183-1192.e1.

18. Belvroy VM, de Beaufort HWL, van Herwaarden JA, et al. Tortuosity of the Descending Thoracic Aorta in Patients with Aneurysm and Type B Dissection. World J Surg https://doi.org/10.1007/s00268-019-05328-w.

19. McNamara JR, Fulton GJ, Manning BJ. Three-dimensional computed tomographic reconstruction of the carotid artery: Identifying high bifurcation. Eur J Vasc Endovasc Surg 2015;49:147-53.

20. Choudhry FA, Grantham JT, Rai AT, et al. Vascular geometry of the extracranial carotid arteries: An analysis of length, diameter, and tortuosity. J Neurointerv Surg 2016;8:536-40.

21. Pressley A. Elementary differential geometry. Second edi. London: Springer; 2010.

22. Schuurmann RCL, Kuster L, Slump CH, et al. Aortic curvature instead of angulation allows improved estimation of the true aorto-iliac trajectory. Eur J Vasc Endovasc Surg 2016;51:216-24.

23. Sharp SJ, Poulaliou M, Thompson SG, et al. A review of published analyses of case-cohort studies and recommendations for future reporting. PLoS One 2014;9.

24. Pourier VEC, Welleweerd JC, Kappelle LJ, et al. Experience of a single center in the conservative approach of 20 consecutive cases of asymptomatic extracranial carotid artery aneurysms. Eur J Neurol 2018;25:1285-9.

25. Nunes T, Heuer C, Marshall J, et al. Package 'epiR '. 2018. [Epub ahead of print].

26. de Vet HCW, Terwee CB, Knol DL, et al. When to use agreement versus reliability measures. J Clin Epidemiol 2006;59:1033-9.

27. Kottner J, Audigé L, Brorson S, et al. Guidelines for reporting reliability and agreement studies (GRRAS) were proposed. J Clin Epidemiol 2011;64:96-106. 
28. Tawfik AM, Sobh DM, Gadelhak B, et al. The effect of age and gender on tortuosity of the descending thoracic Aorta. Eur J Radiol 2019;110:54-9.

29. Lauric A, Hippelheuser J, Safain MG, et al. Curvature effect on hemodynamic conditions at the inner bend of the carotid siphon and its relation to aneurysm formation. J Biomech 2014;47:3018-27.

30. Lee AY, Sanyal A, Xiao Y, et al. Mechanical Instability of Normal and Aneurysmal Arteries. J Biomech 2014;47:3868-75.

31. Attigah N, Külkens S, Zausig N, et al. Surgical Therapy of Extracranial Carotid Artery Aneurysms: Long-Term Results over a 24-Year Period. Eur J Vasc Endovasc Surg 2009;37:127-33.

32. Nordanstig J, Gelin J, Jensen N, et al. National experience with extracranial carotid artery aneurysms: Epidemiology, surgical treatment strategy, and treatment outcome. Ann Vasc Surg 2014;28:882-6.

33. van Laarhoven CJHCM, Pourier VEC, Lindgren AE, et al. Co-prevalence of extracranial carotid aneurysms differs between European intracranial aneurysm cohorts. PLoS One 2020;15:1-10.

34. Pourier VEC, de Borst GJ. Which carotid artery aneurysms need to be treated (and how)? J Cardiovasc Surg (Torino) 2016;57:152-7.

35. Belvroy VM, Romarowski RM, van Bakel TMJ, et al. Impact of Aortic Tortuosity on Displacement Forces in Descending Thoracic Aortic Aneurysms. Eur J Vasc Endovasc Surg 2020:1-8. 


\section{Supplemental Material}

\section{Supplemental Material I}

For calculation of the curvature of the central luminal line (CLL) of the carotid artery, the following equation of the extrinsic linear curvature was used ${ }^{1}$ :

$$
\kappa=\frac{\sqrt{\left(z^{\prime \prime} y^{\prime}-y^{\prime \prime} z^{\prime}\right)^{2}+\left(x^{\prime \prime} z^{\prime}-z^{\prime \prime} x^{\prime}\right)^{2}+\left(y^{\prime \prime} x^{\prime}-x^{\prime \prime} y^{\prime}\right)^{2}}}{\left(x^{\prime 2}+y^{\prime 2}+z^{\prime 2}\right)^{\frac{3}{2}}}
$$

To calculate torsion of the CLL, the following equation derived from the theory described by Pressley² was used:

$$
\tau=\frac{x^{\prime \prime \prime}\left(y^{\prime} z^{\prime \prime}-y^{\prime \prime} z^{\prime}\right)+y^{\prime \prime \prime}\left(x^{\prime \prime} z^{\prime}-x^{\prime} z^{\prime \prime}\right)+z^{\prime \prime \prime}\left(x^{\prime} y^{\prime \prime}-x^{\prime \prime} y^{\prime}\right)}{\left(y^{\prime} z^{\prime \prime}-y^{\prime \prime} z^{\prime}\right)^{2}+\left(x^{\prime \prime} z^{\prime}-x^{\prime} z^{\prime \prime}\right)^{2}+\left(x^{\prime} y^{\prime \prime}-x^{\prime \prime} y^{\prime}\right)^{2}}
$$

In which $[x, y, z]$ are the CLL cartesian coordinates, 'is the first derivative, "is the second derivative, and "' is the third derivative.

\section{References}

1. Schuurmann RCL, Kuster L, Slump CH, et al. Aortic curvature instead of angulation allows improved estimation of the true aorto-iliac trajectory. Eur J Vasc Endovasc Surg 2016;51:216-24.

2. Pressley A. Elementary differential geometry. Second edition. London: Springer; 2010. 

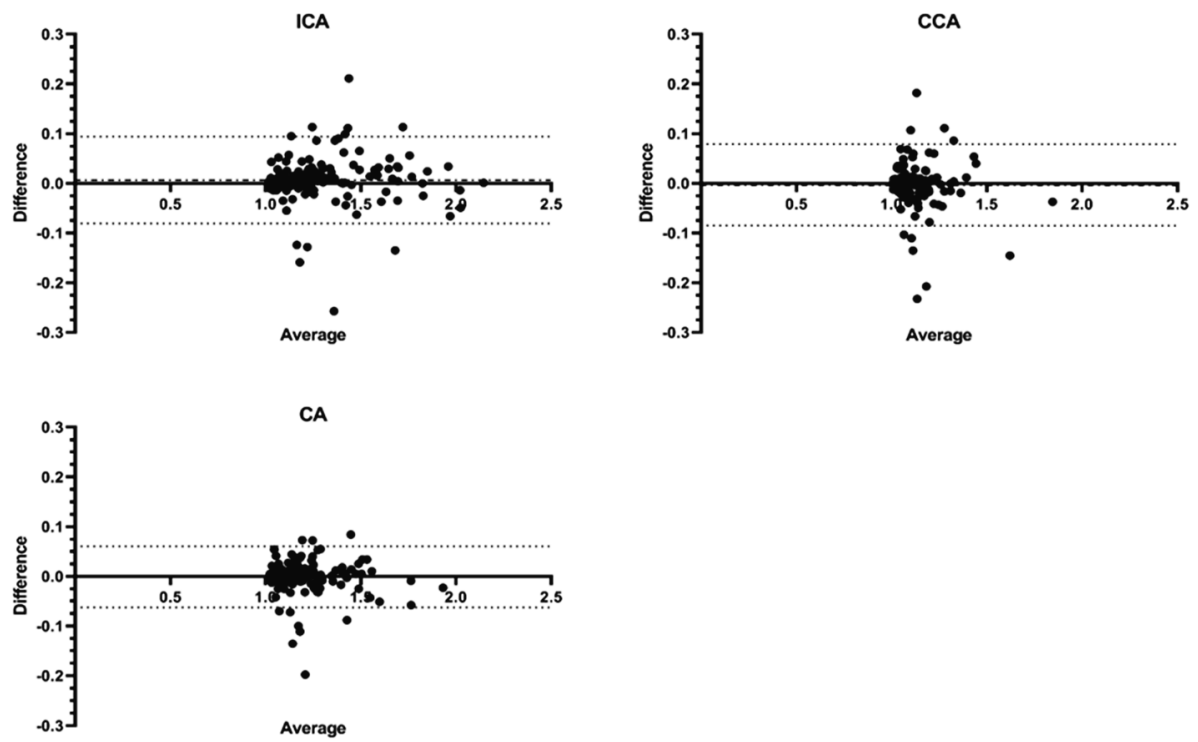

Supplemental Figure I. Bland-Altman plots showing agreement of two operators on tortuosity index (TI) measurements of 175 carotids according to the three subfields (internal carotid artery; ICA, common carotid artery; CCA, total carotid artery; CA). The dash dotted line in the middle represents the mean difference of the TI between the two operators, and the dotted lines represent the upper and lower limits of agreement (mean difference $\pm 1.96 \times$ standard deviation). 

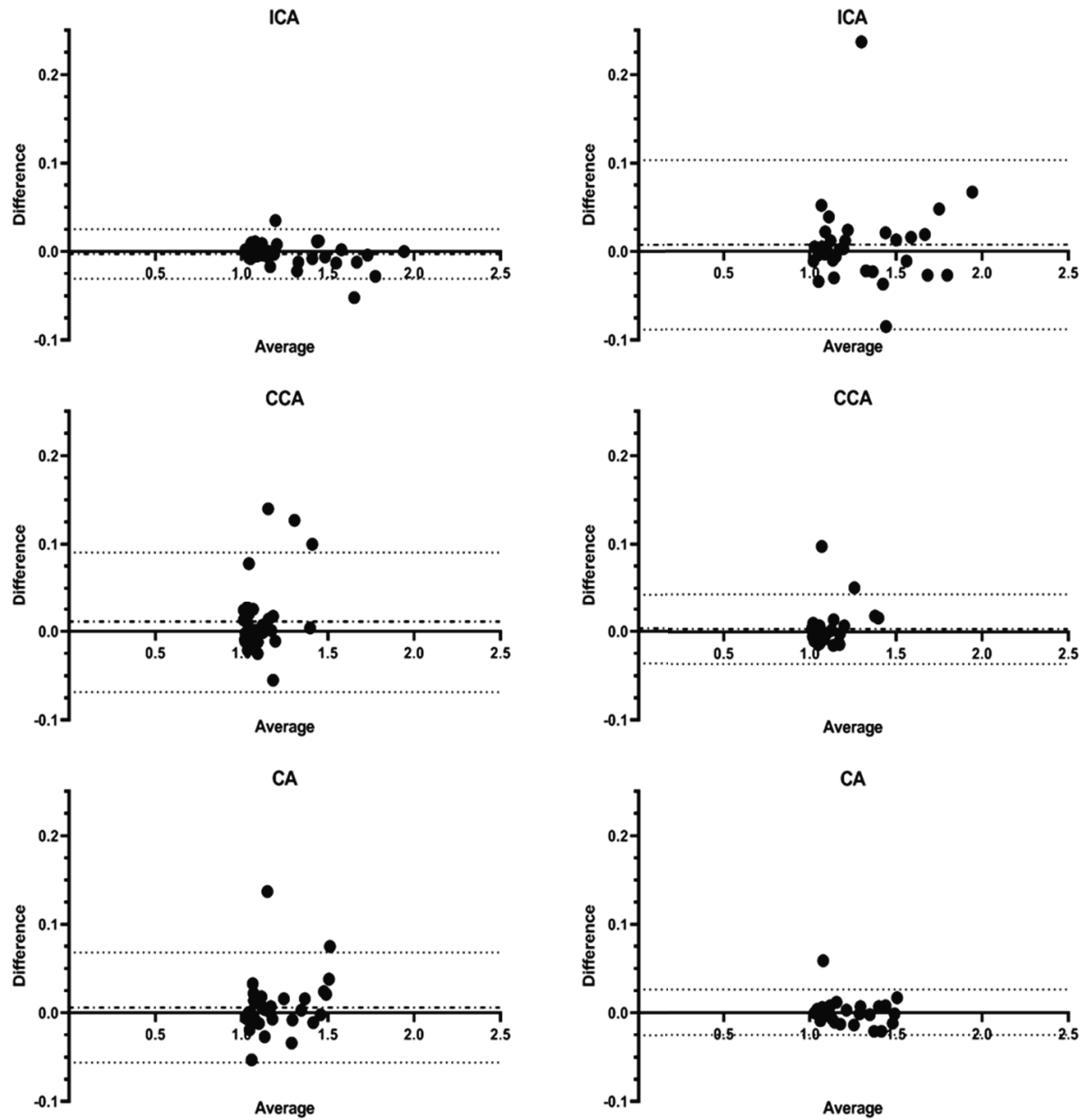

Supplemental Figure II. Bland-Altman plots showing intraoperator agreement of operator 1 (blinded, left panel), and operator 2 (right panel) of the tortuosity index (TI) measurements of 35 carotids according to the three subfields (internal carotid artery; ICA, common carotid artery; CCA, total carotid artery; CA). The dash dotted line in the middle represents the mean difference of the TI between the two operators, and the dotted lines represent the upper and lower limits of agreement (mean difference $\pm 1.96 \times$ standard deviation). 
Supplemental Table I. Intraclass correlation coefficients for both inter- and intraoperator reliability.

\begin{tabular}{|c|c|c|c|c|c|c|}
\hline & \multicolumn{2}{|c|}{$\begin{array}{c}\text { Interoperator } \\
n=175\end{array}$} & \multicolumn{4}{|c|}{$\begin{array}{c}\text { Intraoperator } \\
\quad \mathbf{n}=35\end{array}$} \\
\hline & \multirow[b]{2}{*}{ ICC } & \multirow[b]{2}{*}{$(95 \% \mathrm{CI})$} & \multicolumn{2}{|c|}{ Operator $1^{\star}$} & \multicolumn{2}{|c|}{ Operator 2} \\
\hline & & & ICC & $(95 \% \mathrm{CI})$ & ICC & $(95 \% \mathrm{CI})$ \\
\hline ICA & 0.983 & $(0.977-0.988)$ & 0.998 & $(0.997-0.999)$ & 0.982 & $(0.965-0.991)$ \\
\hline CCA & 0.921 & $(0.849-0.959)$ & 0.978 & $(0.956-0.994)$ & 0.921 & $(0.849-0.959)$ \\
\hline Total CA & 0.980 & $(0.973-0.985)$ & 0.996 & $(0.993-0.998)$ & 0.980 & $(0.962-0.990)$ \\
\hline
\end{tabular}

ICC model: two-way mixed, type: absolute agreement. Abbreviations: $\mathrm{ICC}=$ intraclass correlation coefficient, $\mathrm{CI}=$ confidence interval, $\mathrm{ICA}=$ internal carotid artery, $\mathrm{CCA}=$ common carotid artery, $\mathrm{CA}=$ carotid artery. ${ }^{*}$ indicates blinded operator. 


\section{Part III}

\section{Discussion and summary}

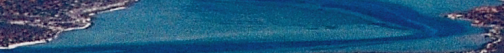
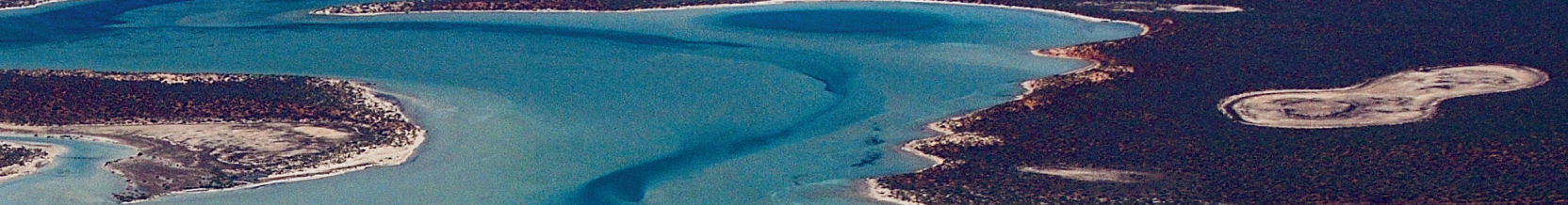

siones

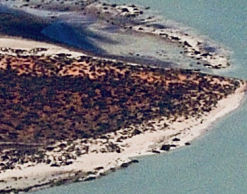

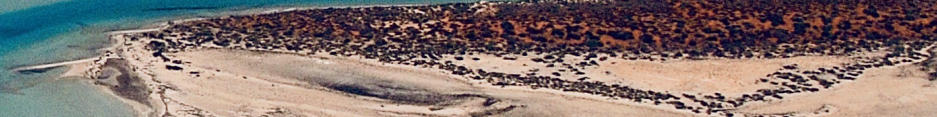

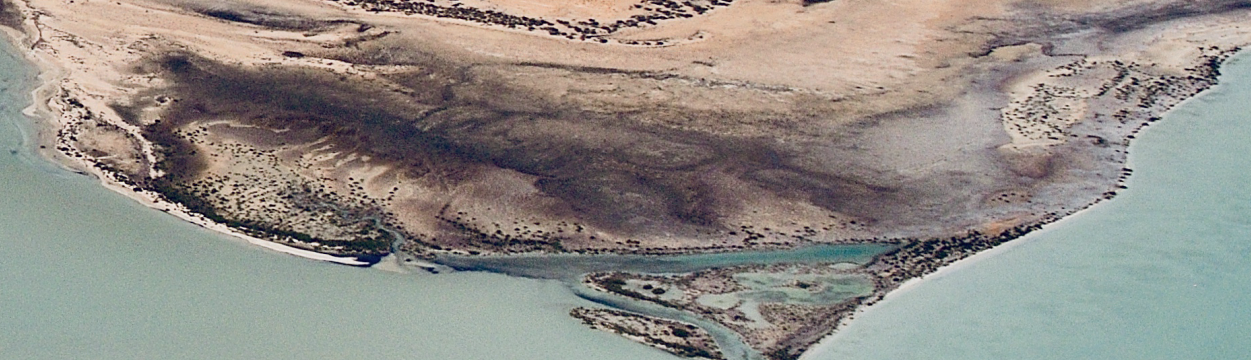





\section{Chapter 10}

Summarizing discussion, future perspectives, and conclusions 


\section{Summarizing discussion}

The present thesis described projects designed to provide insight in contributing etiological factors of aneurysmal disease of the extracranial carotid artery (ECAA). Secondly, novel imaging techniques to monitor patients in the course of ECAA disease were assessed. The ultimate goal of the research discussed in this thesis was to identify clinical and radiological risk factors, and with that improve individualized treatment strategies for ECAA patients in the future. This chapter provides a general discussion and summary of all presented chapters, and subsequent future perspectives and conclusions.

\section{From vascular injury to arterial aneurysm}

Previous histopathological research showed two distinct etiologies of ECAA, postdissection and degenerative alterations of the vascular wall. At this point however, it remains unknown if these categories could be different stages of the same disease ${ }^{1}$. It is estimated that $13-49 \%$ of patients with cervical artery dissection will also develop a dissecting aneurysm ${ }^{2-5}$, predominately in case of a carotid artery dissection ${ }^{6}$. Multi-center data indicated that time of onset varies among dissecting aneurysm patients ${ }^{2}$. As separate analyses for carotid dissecting aneurysms are lacking, we analyzed multi-national data of four European stroke centers that participate in the largest registry available of patients with a carotid artery dissection (the Cervical Artery Dissection and Ischemic Stroke Patients, CADISP, consortium $)^{6,7}$ in chapter 2 . This study showed that approximately one out of five patients with a carotid artery dissection developed a ipsilateral dissecting ECAA. In 52/70 patients, the aneurysm was diagnosed at initial diagnostic work-up after median 6 days (range $0-130$ ). In the remaining eighteen patients (26\%), one quarter of all dissecting ECAA, was shown that ECAA development occurred several months after initial work-up, though the time of diagnosis of ECAA was highly subjective to applied follow-up schemes according to local clinical practice. As prospective long-term followup imaging data of cervical artery dissection patients is lacking, future studies are warranted to monitor future post-dissection cerebral outcome. Prolonged dynamic wall changes were also observed in a prospective MRA study of ten carotid artery dissection patients ${ }^{8}$, in which the mural hematoma in all participants was resolved six months after symptom onset. Chapter 2 showed that an additional intracranial artery dissection was negatively associated, and multiple artery dissections and arterial tortuosity were positively associated with presence of dissecting ECAA. In a previous large CADISP study, multiple artery dissections were associated with an increased risk of cerebral ischemic events at 3 to 6 months after dissection symptom onset, and were suggested as a transient vasculopathy ${ }^{9}$. Taken together, this indicates that carotid artery dissection is a dynamic process with both stenotic and aneurysmal arterial wall changes in following 
days and months after dissection. This process may require more intensive standardized cerebrovascular imaging in the first six months after dissection onset to detect subclinical ischemic events and dynamic configuration changes of the carotids. In this manner, patients at risk - i.e. those who may need more aggressive therapy - can be identified, whereas those without cerebral complications can be dismissed safely from follow-up.

\section{Multiple aneurysms of the cerebrovascular tree}

Arterial aneurysms are known to have common clinical risk- and genetic risk factors. Due to the rarity of ECAA, literature lacks information about associations with aneurysms in other vascular territories. Intracranial aneurysms have been reported to present concomitantly with both carotid stenotic disease ${ }^{10}$ and e.g. aortic aneurysms ${ }^{11,12}$. Therefore, we aimed to analyze co-prevalence of ECAA in intracranial aneurysm patients to gain knowledge about underlying etiology and patient characteristics with concurrent ECAA. As the cerebrovascular tree forms the meeting point of neurology and vascular surgical specialties, we had the unique opportunity to investigate a large cohort of, both ruptured and unruptured, intracranial aneurysm patients from a Dutch tertiary referral center. All cerebrovascular imaging of included patients was reviewed for the presence of a concurrent ECAA. Since no standardized imaging protocol was applied during the study period, we anticipated on this by categorizing cerebrovascular imaging in complete (e.g. CTA carotids, scan levels from heart to top of the brain), and incomplete (e.g. CTA brain, in which scan levels were set from second cervical vertebra until top of the brain). Chapter 3 showed that the co-prevalence of ECAA was 1.9\% in intracranial aneurysm patients with complete cerebrovascular imaging, and only $0.7 \%$. for incompletely imaged patients. In addition, one quarter of identified ECAA patients had additional aneurysms located in the extradural cavernous or the vertebral artery. Since presumed etiology of the aneurysms was unknown in the majority of patients in this observational retrospective study, no further insight in shared risk factors for ECAA and intracranial aneurysms was obtained. In attempt to replicate our findings in another European intracranial aneurysm cohort operating with a standardized imaging protocol including complete cerebrovascular scan levels, we performed a similar co-prevalence study in a large tertiary referral center in Finland in chapter 4. Surprisingly, co-prevalence was reported to be twice as high, and showed that about one out of twenty Finnish intracranial aneurysm patients harbored an ECAA. After adjusting for age, sex, Finnish origin, hypertension, diabetes and statin use, Finnish origin and male sex remained as independent predictors of ECAA presence. The study also showed that at least $25 \%$ of ECAAs would be missed, if the carotid bifurcation was not imaged. Hence carotid imaging including the carotid bifurcation was proposed as reference standard technique to identify ECAA in intracranial aneurysms patients. Although the co-prevalence estimates of ECAA in intracranial patients is not remarkably high and screening seems unnecessary at this point, insights in clinical risk 
profiles of more prevalent aneurysms may be worthwhile to understand and extent knowledge of aneurysmal pathology in other arteries. Moreover, the question arises if co-prevalence estimates can be used reversibly, and if so, may indicate both aneurysms are part of the same underlying disease. Future imaging studies with preferably similar indication, scan levels, and modality should be carried out, in order to assess aneurysmal disease of the cerebrovascular tree. Multi-center research collaborations in the international Carotid Aneurysm Registry will give insight on natural history and prognosis of patients with these rare aneurysms, which can be implemented in daily clinical practice.

\section{Arterial aneurysms as systemic disease}

In general, arterial aneurysms are considered as focal manifestations of a systemic vascular condition, and various studies report co-prevalence of aneurysms in different vascular beds. The European and American Society for Vascular Surgery updated guidelines for management of abdominal aortic aneurysm (AAA) recommend additional AAA aneurysm screening in patients with a true peripheral aneurysm (IIb, level C) ${ }^{13}$, more especially femoral or popliteal artery aneurysm(I, level A $)^{14}$. The guidelines lack, however, a clear overview of the risk profile for patients at risk for concurrent aneurysms, and type and extensiveness of aneurysm evaluation is left to the readers' interpretation. The clinical implementation of these recommendations could therefore vary among treating physicians. The European guideline for acute limb ischemia is more directive, as in patients treated for a thrombosed popliteal aneurysm, duplex ultrasound imaging every three years of the treated and contralateral arteries, as well as of the aorta, iliac, and femoral arteries, should be considered (IIa, level C) ${ }^{15}$. In order to provide an overview of reported co-prevalence of different arterial aneurysms throughout the vasculature, and potentially indicate putative clinical risk factors for multiple aneurysms, the systematic review in chapter 5 was undertaken. The study showed that approximately one out of six patients with a primary aneurysm harbors a concurrent aneurysm, and one in four if the patient has a popliteal artery aneurysm. More than 19 combinations of different aneurysms were described. The clinical risk for concurrent aneurysms was higher in case of increased age, hypertension, stenotic disease, presence of $\geq 3$ aneurysms and size of the primary aneurysm. However, the supporting evidence was not ambiguous due to the observed heterogeneity in study properties. In addition, reversibility of aneurysm coprevalence estimates among two types of arteries was not convincing in chapter 5 . The observed differences in estimates may well have been caused by differences in aneurysm definition or included patient and their clinical characteristics. Ideally, prospective studies with a similar modality, e.g. duplex ultrasound, should be performed in which both regions of interest are screened in all participants, and time-to-diagnosis is included when crystalizing clinical risk. Radiation exposure should be minimized in selecting type 
of modality and time interval. Nevertheless, based on this review, treating physicians should consider additional aneurysm screening in typical vascular patients (elderly male with hypertension and stenotic disease), and in case of presence of ( $\geq 3)$ multiple aneurysms and large size of the primary aneurysm. In particular female AAA patients seem more susceptible for additional thoracic aortic aneurysm or intracranial aneurysm. Future analyses should focus on the health-related burden if the concurrent aneurysm diagnosed at screening is left untreated. Besides cost-benefits assessed by simulation studies ${ }^{16}$, health related quality of life assessment ${ }^{17}$ should be taken into account in order to develop well-considered screening algorithms.

\section{Genetic susceptibility as surrogate marker for aneurysm diameter}

Next to clinical risk factors, several genetic studies have been conducted to elucidate the strong heritability observed in particular AAA. In the past decade, genome-wide association studies (GWAS) have uncovered ten common genetic risk variants, i.e. singlenucleotide polymorphisms, associated to AAA susceptibility ${ }^{18}$. On the other hand, evidence is emerging that many complex diseases are suggested to be polygenic in origin, comprising small effects of hundreds or even thousands of common variants that in aggregate, explain a substantial proportion of disease susceptibility and heritability ${ }^{19-21}$. In chapter 6, we have analyzed currently available genetic knowledge for AAA with data of the Aneurysm-Express Biobank Study ${ }^{22}$. The biobank cohort consisted of patients that underwent open surgical treatment for clinically manifested AAA or peripheral aneurysms (i.e. popliteal, iliac, femoral and carotid). A best-fit polygenic risk score was modelled based on previous GWAS effect estimates ${ }^{23}$, and tested for association with clinical phenotypes. Although both currently available AAA-GWAS (4,972 cases and 99,858 controls $\left.^{23}\right)$ data, and sample size $(n=415)$ used in this genetic study were modest, chapter 6 demonstrates that polygenic scores of AAA susceptibility were associated with diameter of the aneurysm sac in patients with aneurysms in different vascular beds. The potential clinical utility of polygenic approaches in aneurysm patients should be further validated in larger cohorts with standardized sample collection. Future studies should therefore focus on biobank collaborations to facilitate the in-depth scrutiny and replication of genetic susceptibility on aneurysm and AAA initiation and progression, as well as the exploration of gender differences. In the future, polygenic risk scores could assist in selecting high-risk patients for adverse outcomes such as aneurysm rupture or aneurysm-related symptoms in addition to established clinical risk factors. Genetic data will aid in creating personalized medicine for aneurysmal disease as observed in coronary artery disease and PCSK9 (proprotein convertase subtilisin/kexin type 9) inhibition in secondary prevention ${ }^{24}$, and absolute risk stratification in breast cancer ${ }^{25}$. 


\section{Protocol for volumetric ECAA assessment}

Imaging software have become critical tools in the diagnosis and follow-up of aneurysms. Monitoring of aneurysm size is currently assessed by measuring the bi-directional maximum aneurysm diameter from outer to outer vessel wall on duplex ultrasound, CTA or MRA imaging techniques. In general, asymptomatic, non-growing ECAA are generally treated conservatively ${ }^{26}$, while surgical or endovascular aneurysm exclusion may be considered in case of symptomatic or growing aneurysms ${ }^{27}$. Also ECAA size is currently measured by outer-outer bi-directional maximum aneurysm diameter, extracted from the well-established approach for AAA ${ }^{13,14}$. Nevertheless, ECAAs may be present in a large variety of shapes and sizes, and maximum aneurysm diameters between 4 and $60 \mathrm{~mm}$ have been reported ${ }^{26,28}$. It seems reasonable that growth may also occur in other directions than just obliquely, and conventional diameter measurements might therefore be inadequate to indicate geometrical differences. Therefore, we proposed a protocol to assess volume of the aneurysm sac of the ECAA in chapter 7. An expert panel of vascular surgeons was consulted in the conceptualization of the volumetric protocol. Volumes were measured through manual carotid segmentations by two independent operators. The reproducibility and similarity measures of the CTA-based manual segmentations according to the volumetric protocol were excellent, and this study was a step-up to a future semi-automatic volumetric tool. The next step would entail programming an algorithm based on the manual segmentations of the studied 40 ECAA images, and further testing and fine-tuning the algorithm in a large and possibly augmented dataset. Future (semi-)automatic software will minimize the time required to perform volumetric assessment and bypass the need for skilled operators. The increasing scientific knowledge of neural networks and artificial intelligence will accelerate these developments. Semi- or fully automatic software are also being developed in other vascular territories, such as aortic $^{29}$ and intracranial arteries ${ }^{30}$. Next to aneurysm volume, vascular wall characteristics such as calcifications and thrombus, that may be surrogate markers for aneurysm wall instability, will be simultaneously generated. The ongoing challenge in fine-tuning volumetric software is to adapt the algorithm or so-called pipeline to the large variety in aneurysm morphology, and the discrimination of diseased arterial wall and surrounding tissue. This field is evolving rapidly, and will primarily compliment conventional diameter assessment. Indeed, automatic volumetric software will have the potential to replace therapy decision thresholds based on maximum diameter.

\section{Aneurysm wall imaging for prediction of outcome}

In chapter 83.0T MR imaging of the carotid wall in asymptomatic ECAA patients was used to see whether baseline imaging characteristics correlated with aneurysm growth or cerebral complications. In this explorative study, we investigated if ECAA wall imaging with MR was feasible, and if gadolinium $(\mathrm{Gd})$ contrast enhancement was related to 
outcome. It is believed that Gd-enhancement reflects both density of the vasa vasorum and endothelial permeability, and can indicate local inflammation of the vessel wall ${ }^{31-33}$. ECAA growth was defined as at least $2.0 \mathrm{~mm}$ increase of maximum diameter measured double oblique from outer to outer wall, and cerebral outcome was assessed in terms of infarcts and white matter lesions. The study showed that the majority of patients exhibited Gd-enhancement at baseline, while aneurysm growth occurred in approximately one quarter of all patients, both with and without wall-enhancement. Even though ECAA wall imaging is feasible with good inter- and intra-rater reproducibility, future wellpowered studies are needed to assess Gd-enhancement as surrogate marker for ECAA wall instability. However, it was striking that these relatively young (median age 55 years), and asymptomatic ECAA patients showed often a high (bilateral) white matter lesion score compared to an otherwise healthy population ${ }^{34}$. This finding does not suggest so much a causal relationship of ECAA and white matter lesions, but points to a shared etiology for the presence of both diseases. White matter lesions are used as a marker for small vessel disease ${ }^{35}$ and have been associated with an increased risk of future stroke and cognitive decline $e^{35,36}$. Future long-term cerebral outcome in ECAA patients should be assessed, and perhaps medical therapy should be optimized further, not only to prevent possible complications due to thrombo-emboli, but also to treat risk factors of underlying generalized vascular disease. The applied MR protocol in chapter $\mathbf{8}$ was based on intracranial aneurysms ${ }^{37}$ in which Gd-enhancement seemed predictive of aneurysm growth $^{38}$. Since healthy intracranial arteries lack adventitial vasa vasorum, Gdenhancement of intracranial arteries may indicate stronger local pathological neovascularization and thus inflammation ${ }^{39}$. Discrimination in enhancement of normal or pathological vasa vasorum of the extracranial carotid arteries remains challenging at this point in time. In addition, slow flow within relatively large ECAAs could mimic wall-enhancement, and thus affect the validity and clinical applicability of this study. Future optimization of the MR scanning protocol specified for the extracranial carotid artery and comparison with healthy arteries should be carried out. Next, well-powered studies are needed to indicate if Gd-enhancement identifies patients at risk for aneurysm growth or cerebral complications. Ideally Gd-enhancement can aid the selection of highrisk ECAA patients possibly benefit from invasive treatment whereas follow-up imaging can be scaled back in conservatively treated low-risk patients.

\section{Quantitative assessment of arterial tortuosity}

Previous literature has suggested that arterial tortuosity is associated with arterial dissection, and that intima disruption of the carotid artery at its turn is a common cause of ECAA ${ }^{1}$ as is described in chapter 2. If arterial tortuosity is also associated with ECAA, independent of presence of carotid artery dissection, this may be integrated in a screening algorithm for ECAA. Previous research geometrically differentiated between carotid 
loops, S- and C-bends ${ }^{43}$, which were interpreted from angiographies from different modalities. Since 2D images for evaluation of arterial geometry are highly subjective to visual projection of the angiography (e.g. derived from digital subtraction angiography), more reliable quantitative estimates indicating severity of tortuosity can be obtained by 3D reconstruction, such as tortuosity index (TI) $)^{41,42,44-47}$. TI was defined as the ratio of the length of curved, or central luminal line (CLL), and the straight-line length between two anatomical landmarks ${ }^{44,45,48}$. Besides differences in length, we also included curvature and torsion to indicate geometry ${ }^{49-51}$. Curvature was defined as the degree a line deviates from being straight, and torsion expresses the direction and degree of turns of a line. In chapter 9, we conducted a cross-sectional case-control study to investigate if a high degree of carotid tortuosity was associated with ECAA, and excluded patient with presence or history of carotid dissection. Thin-slice CTA scans were analyzed according to a predefined protocol with software of 3mensio Vascular and an in-house MATLAB based script. In different subfields the TI was measured: the internal carotid artery (ICA), common carotid artery (CCA), and entire carotid artery from CCA origin in the aortic arch up to skull base. Curvature and torsion were categorized in low, medium, and high, and expressed in counts of these categories across the CLL of the entire carotid artery. Chapter 9 showed also that carotid tortuosity was similar in both carotids within ECAA patients, though significantly higher in comparison with age- and sex-matched controls. Presence of ECAA and higher age were independently associated with arterial tortuosity. Ideally, carotid tortuosity could be used as an easy-assessable screening marker for ECAA, independently of presence of carotid arterial dissection. Confounding by previous arterial dissection is however not completely ruled out, since obtaining a complete dissection-free cohort is challenging as patients can be asymptomatic and radiological identification is hampered by time-dependent vascular remodeling. Nevertheless, this study showed that carotid tortuosity may be high in ECAA patients and it is imaginable that carotid tortuosity hampers endovascular treatment options due to hostile landing zones of the stent graft. The effect of tortuosity on endovascular stenting was earlier studied in thoracic aneurysm patients. By use of computational hemodynamics studies, impressive displacement forces after TEVAR were observed in patients with thoracic tortuosity ${ }^{52}$. Even though the blood pressure is likely to be lower within the carotids, prior tortuous anatomy could relatively mimic displacement forces after carotid stenting and cause migration of the stent. Future studies in which arterial tortuosity is quantitatively assessed in patients treated with endovascular stenting with and without technical success, and in patients regarded suitable and unsuitable for endovascular approaches need to be performed in order to define a quantitative tortuosity cut-off value for endovascular therapy. In this way ECAA patients suitable for endovascular techniques are being identified, and iatrogenic complications and re-interventions may be prevented. Moreover, future computational hemodynamics studies in ECAA patients could also 
optimize stent graft design for tortuous carotids, and may elucidate altered blood flow patterns by ECAA presence susceptible for e.g. clotting or peak pressure areas with expanding vascular remodeling as a consequence.

\section{Future perspectives for ECAA}

The following paragraph illustrates my personal perspectives for the future diagnostic work-up, follow-up and treatment of ECAA patients, derived from acquired knowledge during my PhD.

From my point of view, ECAA can be seen as a marker for experienced carotid injury at a certain point and like any other process in the body, inflammatory responses opted to restore the vascular hemostasis. One may argue the healing response was either exaggerated or (still is) imbalanced while the ECAA is present. The clinical presentation of patients with ECAA ranges from small to relatively large size, and asymptomatic to symptomatic ECAA. It is likely that size is related to symptoms, though (sub)clinical cerebral events are also observed in small (i.e. below $12 \mathrm{~mm}$ ) ECAA. The ultimate goal of ECAA treatment is the prevention of cerebral sequelae, but related cerebral outcomes such as infarcts and multifactorial white matter lesions seem in many cases more subtle than thromboembolic events observed from instable stenotic plaques. Moreover, as thrombus is very often absent in the aneurysm, the direct causal relation with major cerebral presenting events seems hard to justify and further source examination should be carried out. Long-term assessment of ECAA related cerebral outcome from patients included in the observational Carotid Aneurysm Registry is needed in order to comprehend its clinical relevance.

The focus of future ECAA research should therefore be the identification of patients with a high- and low-risk ECAA with dedicated follow-up tools, as risk stratification is crucial in the development of future clinical algorithms. Substantial insight in different treatment strategies and associated clinical outcome can be provided by future analyses from the international Carotid Aneurysm Registry. In addition, innovations in imaging software are warranted in order to quantitatively monitor vascular wall and associated cerebral outcome. Ideally, outpatient follow-up would be performed following standardized CT or MR imaging protocols complimented by software in which suitable 3D parameters are measured, such as aneurysm growth based on volume, location of expansion, altered aneurysm wall components, flow patterns to visualize pro-thrombotic areas and peak wall stresses, and lastly monitor cerebral ischemic changes. These applications could additionally be translated to other vascular territories, to overcome the costs of 
development. Type of modality and time intervals should be well-considered in order to minimize cumulative radiation exposure. Subsequently, intensity of follow-up imaging can be scaled back in patients that benefit most from a conservative approach, and sophisticated follow-up tools will assist in determining treatment options in patients that warrant surgical therapy.

Besides radiological characterization, genetic profiling could elucidate if all ECAA patients are reflections of different phases from the same underlying vasculopathy. Common pathophysiological pathways will ideally offer targets for preventive therapy next to available symptomatic therapy. In addition, polygenic susceptibility for clinical endpoints such as aneurysm growth or cerebral ischemia could assist in further identification of high-risk patient groups. Outpatient clinic biobank sampling should also be performed at international level to obtain a sufficient sample size within the near future.

\section{Conclusions}

- Insight in clinical risk profile of aneurysms seems worthwhile to understand and extent knowledge of aneurysmal pathology in other arteries. Future clinical implementation of aneurysm screening algorithms should be based on the health-related burden when the concurrent aneurysm at screening is left untreated.

- Clinical, radiological, and genetic risk features of ECAA can be deployed to identify those at high-risk for aneurysm deterioration and related cerebral outcome. Risk stratification models using these features are essential in determining optimal individualized medicine.

- Further optimization of (semi-)automatic imaging software are warranted in order to quantitatively assess aneurysm wall features and associated cerebral outcome.

- The future focus of the international Carotid Aneurysm Registry should be the identification of patients with an ECAA with high- and low-risk for adverse outcome. Insight in long-term follow-up by use of dedicated imaging tools in addition to clinical and genetic risk profile will result in future tailored therapy for ECAA patients. 


\section{References}

1. Welleweerd JC, Nelissen BGL, Koole D, et al. Histological analysis of extracranial carotid artery aneurysms. PLoS One 2015;10:1-9.

2. Larsson SC, King A, Madigan J, et al. Prognosis of carotid dissecting aneurysms. Neurology 2017;88:646-52.

3. Daou B, Hammer C, Chalouhi N, et al. Dissecting pseudoaneurysms: Predictors of symptom occurrence, enlargement, clinical outcome, and treatment. J Neurosurg 2016;125:936-42.

4. Guillon B, Brunereau L, Biousse V, et al. Long-term follow-up of aneurysms developed during extracranial internal carotid artery dissection. Neurology 1999;53:117-22.

5. Touzé E, Randoux B, Méary E, et al. Aneurysmal Forms of Cervical Artery Dissection, associated factors and outcome. Stroke 2001;32:418-23.

6. Debette S, Grond-Ginsbach C, Bodenant M, et al. Differential features of carotid and vertebral artery dissections The CADISP study. Neurology 2011;77:1174-81.

7. Debette S, Metso TM, Pezzini A, et al. CADISP-genetics: An International project searching for genetic risk factors of cervical artery dissections. Int J Stroke 2009;4:224-30.

8. Heldner MR, Nedelcheva M, Yan X, et al. Dynamic changes of intramural hematoma in patients with acute spontaneous internal carotid artery dissection. Int J Stroke 2015;10:887-92.

9. Compter A, Schilling S, Vaineau CJ, et al. Determinants and outcome of multiple and early recurrent cervical artery dissections. Neurology 2018;91:e769-80.

10. Radak D, Sotirovic $V$, Tanaskovic $S$, et al. Intracranial aneurysms in patients with carotid disease: Not so rare as we think. Angiology 2014;65:12-6.

11. Kurtelius A, Väntti N, Rezai Jahromi B, et al. Association of Intracranial Aneurysms With Aortic Aneurysms in 125 Patients With Fusiform and 4253 Patients With Saccular Intracranial Aneurysms and Their Family Members and Population Controls. J Am Heart Assoc 2019;8:e13277.

12. Rouchaud A, Brandt MD, Rydberg AM, et al. Prevalence of intracranial aneurysms in patients with aortic aneurysms. Am J Neuroradiol 2016;37:1664-8.

13. Wanhainen A, Verzini F, Van Herzeele I, et al. Editor's Choice - European Society for Vascular Surgery (ESVS) 2019 Clinical Practice Guidelines on the Management of Abdominal Aorto-iliac Artery Aneurysms. Eur J Vasc Endovasc Surg 2019;57:8-93.

14. Chaikof EL, Dalman RL, Eskandari MK, et al. The Society for Vascular Surgery practice guidelines on the care of patients with an abdominal aortic aneurysm. J Vasc Surg 2018;67:2-77.e2.

15. Björck M, Earnshaw JJ, Acosta S, et al. Editor's Choice - European Society for Vascular Surgery (ESVS) 2020 Clinical Practice Guidelines on the Management of Acute Limb Ischaemia. Eur J Vasc Endovasc Surg 2020;59:173-218.

16. Ball BZ, Jiang B, Mehndiratta $\mathrm{P}$, et al. Screening individuals with intracranial aneurysms for abdominal aortic aneurysms is cost-effective based on estimated coprevalence. J Vasc Surg 2016;64:811-818.e3.

17. Lyttkens L, Wanhainen A, Svensjö S, et al. Systematic Review and Meta-Analysis of Health Related Quality of Life and Reported Experiences in Patients With Abdominal Aortic Aneurysm Under Ultrasound Surveillance. Eur J Vasc Endovasc Surg 2020;59:420-7.

18. Jones GT, Tromp G, Kuivaniemi H, et al. Meta-Analysis of Genome-Wide Association Studies for Abdominal Aortic Aneurysm Identifies Four New Disease-Specific Risk Loci. Circ Res 2017;120:341-53.

19. Euesden J, Lewis CM, Reilly PFO. Genome analysis PRSice : Polygenic Risk Score software. 2015;31:1466-8.

20. Torkamani A. The personal and clinical utility of polygenic risk scores. Nat Rev Genet 2018;19:581-90.

21. Chatterjee N, Shi J, García-Closas M. Developing and evaluating polygenic risk prediction models for stratified disease prevention. Nat Rev Genet 2016;17:392-406.

22. Hurks R, Hoefer IE, Vink A, et al. Aneurysm-express: Human abdominal aortic aneurysm wall expression in relation to heterogeneity and vascular events-rationale and design. Eur Surg Res 2010;45:34-40.

23. Loci NDR, Jones GT, Tromp G, et al. Meta-Analysis of Genome-Wide Association Studies for Abdominal Aortic Aneurysm Identifies Four. https://doi.org/10.1161/CIRCRESAHA.116.308765.

24. Damask A, Steg PG, Schwartz GG, et al. Patients With High Genome-Wide Polygenic Risk Scores for Coronary Artery Disease May Receive Greater Clinical Benefit From Alirocumab Treatment in the ODYSSEY OUTCOMES Trial. Circulation 2020;141:624-36.

25. Maas P, Barrdahl M, Joshi AD, et al. Breast Cancer Risk From Modifiable and Nonmodifiable Risk Factors Among White Women in the United States. JAMA Oncol 2016;2:1295-302.

26. Pourier VEC, Welleweerd JC, Kappelle LJ, et al. Experience of a single center in the conservative approach of 20 consecutive cases of asymptomatic extracranial carotid artery aneurysms. Eur J Neurol 2018;25:1285-9. 
27. Pourier VEC, de Borst GJ. Which carotid artery aneurysms need to be treated (and how)? J Cardiovasc Surg (Torino) 2016;57:152-7.

28. Nordanstig J, Gelin J, Jensen N, et al. National experience with extracranial carotid artery aneurysms: Epidemiology, surgical treatment strategy, and treatment outcome. Ann Vasc Surg 2014;28:882-6.

29. Lareyre F, Adam C, Carrier M, et al. A fully automated pipeline for mining abdominal aortic aneurysm using image segmentation. Sci Rep 2019;9:1-14.

30. Park A, Chute C, Rajpurkar P, et al. Deep Learning-Assisted Diagnosis of Cerebral Aneurysms Using the HeadXNet Model. JAMA Netw open 2019;2:e195600.

31. Aoki S, Aoki K, Ohsawa S, et al. Dynamic MR imaging of the carotid wall. J Magn Reson Imaging 1999;9:420-7.

32. Qiao Y, Etesami M, Astor BC, et al. Carotid plaque neovascularization and hemorrhage detected by MR imaging are associated with recent cerebrovascular ischemic events. Am J Neuroradiol 2012;33:755-60.

33. Kerwin WS, O'Brien KD, Ferguson MS, et al. Plaque enhancement 2006 Carotid_plaque inflammation in carotid artery. Radiology 2006;241:459-68.

34. Leeuw F de, Groot J de, Achten E, et al. Prevalence of cerebral white matter lesions in elderly people: A population based magnetic resonance imaging study: The Rotterdam Scan Study. J Neurol Neurosurg Psychiatry 2001;70:9-14.

35. Van Dijk EJ, Prins ND, Vrooman HA, et al. Progression of cerebral small vessel disease in relation to risk factors and cognitive consequences: Rotterdam scan study. Stroke 2008;39:2712-9.

36. Debette S, Schilling S, Duperron MG, et al. Clinical Significance of Magnetic Resonance Imaging Markers of Vascular Brain Injury: A Systematic Review and Meta-analysis. JAMA Neurol 2019;76:81-94.

37. Dieleman N, Yang W, Van Der Kolk AG, et al. Qualitative evaluation of a high-resolution 3D multi-sequence intracranial vessel wall protocol at 3 tesla MRI. PLoS One 2016;11:1-11.

38. Vergouwen MDI, Backes D, Van Der Schaaf IC, et al. Gadolinium enhancement of the aneurysm wall in unruptured intracranial aneurysms is associated with an increased risk of aneurysm instability: A follow-up study. Am J Neuroradiol 2019;40:1112-6.

39. Portanova A, Hakakian N, Mikulis DJ, et al. Intracranial vasa vasorum: Insights and implications for imaging. Radiology 2013;267:667-79.

40. Saba L, Argiolas GM, Sumer S, et al. Association between internal carotid artery dissection and arterial tortuosity. Neuroradiology 2015;57:149-53.

41. Giossi A, Mardighian D, Caria F, et al. Arterial tortuosity in patients with spontaneous cervical artery dissection. Neuroradiology 2017;59:571-5.

42. Kim BJ, Yang E, Kim NY, et al. Vascular Tortuosity May Be Associated with Cervical Artery Dissection. Stroke 2016;47:2548-52.

43. Zenteno M, Viñuela F, Moscote-Salazar LR, et al. Clinical implications of internal carotid artery tortuosity, kinking and coiling: a systematic review. Rom Neurosurg 2014;21:51-60.

44. de Vries EE, Pourier VEC, van Laarhoven CJHCM, et al. Comparability of semiautomatic tortuosity measurements in the carotid artery. Neuroradiology 2019;61:147-53.

45. Chen CK, Chou HP, Guo CY, et al. Interobserver and intraobserver variability in measuring the tortuosity of the thoracic aorta on computed tomography. J Vasc Surg 2018;68:1183-1192.e1.

46. McNamara JR, Fulton GJ, Manning BJ. Three-dimensional computed tomographic reconstruction of the carotid artery: Identifying high bifurcation. Eur J Vasc Endovasc Surg 2015;49:147-53.

47. Belvroy VM, de Beaufort HWL, van Herwaarden JA, et al. Tortuosity of the Descending Thoracic Aorta in Patients with Aneurysm and Type B Dissection. World J Surg https://doi.org/10.1007/s00268-019-05328-w.

48. Choudhry FA, Grantham JT, Rai AT, et al. Vascular geometry of the extracranial carotid arteries: An analysis of length, diameter, and tortuosity. J Neurointerv Surg 2016;8:536-40.

49. Lauric A, Safain MG, Hippelheuser J, et al. High curvature of the internal carotid artery is associated with the presence of intracranial aneurysms. J Neurointerv Surg 2014;6:733-9.

50. Pressley A. Elementary differential geometry. Second edi. London: Springer; 2010.

51. Schuurmann RCL, Kuster L, Slump CH, et al. Aortic curvature instead of angulation allows improved estimation of the true aorto-iliac trajectory. Eur J Vasc Endovasc Surg 2016;51:216-24.

52. Belvroy VM, Romarowski RM, van Bakel TMJ, et al. Impact of Aortic Tortuosity on Displacement Forces in Descending Thoracic Aortic Aneurysms. Eur J Vasc Endovasc Surg 2020:1-8. 



\section{Chapter 11}

Summary in Dutch | Nederlandse samenvatting 


\section{Inleiding}

Een vaatverwijding (aneurysma) van de halsslagader (arteria carotis) is een zeldzame aandoening. Slechts $2 \%$ van alle halsslagaderoperaties die jaarlijks in een ziekenhuis plaatsvinden, wordt verricht vanwege een extracranieel (buiten de schedel) carotis aneurysma. Normaal gesproken heeft ieder mens twee halsslagaders welke de hersenen van bloed voorzien, aan de linker- en rechterkant. De diameter van het bloedvat bij vrouwen bedraagt $6.0 \mathrm{~mm}$ en bij mannen $6.5 \mathrm{~mm}$. De tak die richting de hersenen gaat (arteria carotis interna) is smaller, namelijk respectievelijk $4.5 \mathrm{~mm}$ en $6.0 \mathrm{~mm}$. Zodra zich een lokale verzwakking in de binnenwand van de halsslagader ontwikkelt, kan hier een vaatverwijding of aneurysma ontstaan. In de literatuur staan diameters van extracraniële carotis aneurysmata beschreven tussen de $4.0-60.0 \mathrm{~mm}$ en deze aneurysmata zijn veelal gelokaliseerd in de arteria carotis interna (Figuur 1). De precieze oorzaak van een extracranieel carotis aneurysma is vaak onbekend, maar door histologisch onderzoek weten we dat onderliggende bloedvatziekten een grote rol spelen, bijvoorbeeld slagaderverkalking (atherosclerose), bindweefselziekten en een spontane of traumatische scheur (dissectie) in de binnenste laag van de vaatwand.

De klachten die patiënten met een extracranieel carotis aneurysma ervaren lopen ver uiteen. Ongeveer de helft van de patiënten op de polikliniek van het Hart \& vaatcentrum ervaart geen klachten. De andere helft ervaart een vorm van lokale symptomen, zoals het gevoel van een massa in de hals, slik- of stemproblemen. Een nog kleiner gedeelte van de patiënten ervaart tijdelijk of langdurig functieverlies van de hersenen. De locatie, grootte en oorzaak van het extracraniële carotis aneurysma bepalen of een patiënt klachten ontwikkelt. Er zijn verschillende manieren om een extracranieel carotis aneurysma te behandelen. Globaal bestaat de keuze uit afwachten, conservatief of invasief behandelen. Bij een afwachtend beleid worden patiënten gecontroleerd met behulp van beeldvorming. De conservatieve behandeling bestaat uit een combinatie van controle door beeldvorming en medicijnen om de bloedvaten zo gezond mogelijk te houden en stolling van bloed te voorkomen. Daarnaast zijn er verschillende invasieve of operatieve behandelingen om het aneurysma te verwijderen of uit te sluiten van de bloedbaan. Vanwege de zeldzaamheid van de ziekte bestaat er op dit moment geen richtlijn die aangeeft welke patiënt welke behandeling moet ondergaan. Ondanks het ontbreken van een optimale behandelingsstrategie, wordt de groei van het aneurysma of symptomen gezien als indicatie voor een invasieve behandeling. De afdeling Vaatchirurgie van het Universitair Medisch Centrum Utrecht heeft in 2014 de internationale Carotis Aneurysma Registratie opgezet om patiënten met een extracranieel carotis aneurysma en de daarbij gekozen behandeling, te volgen in de tijd. Op dit moment zijn er 415 patiënten geïncludeerd afkomstig uit meer dan 30 (inter)nationale ziekenhuizen (d.d. april 2020). 
In de toekomst zal deze registratie duidelijkheid geven over de verschillende patiënt- en aneurysmakarakteristieken, de meest geschikte behandeling en de resultaten hiervan. Daarnaast vormt deze registratie de basis voor dit proefschrift waarin verschillende aspecten worden belicht om de zorg voor patiënten met een extracranieel carotis aneurysma te verbeteren. De focus van het proefschrift is de identificatie van zowel klinische als radiologische risicofactoren, die in de toekomst gebruikt kunnen worden om te bepalen welke patiënten een behandeling nodig hebben.

\section{Risicofactoren voor het ontstaan van aneurysmata}

Het tijdspunt waarop een extracranieel carotis aneurysma gediagnosticeerd wordt, in patiënten die een carotis dissectie hebben doorgemaakt, is in hoofdstuk $\mathbf{2}$ beschreven. De studie toont aan dat het moment van ontstaan van extracraniële carotis aneurysmata na dissectie van de halsslagader, verschilt van dagen tot maanden nadat de eerste dissectie gerelateerde symptomen aanwezig waren. In ongeveer $25 \%$ van de gevallen wordt het extracraniële carotis aneurysma pas bij herhaalde follow-up beeldvorming gedetecteerd. Daarnaast blijkt dat zowel arteriële tortuositeit (vele bochten) als meerdere dissecties in de cervicale slagaders geassocieerd zijn met het ontstaan van een extracranieel carotis aneurysma. Het belang van het monitoren van de (stille) cerebrale gevolgen wordt aangegeven, die ontstaan door het dynamische herstelproces van de arteria carotis in het eerste half jaar na start van de dissectie-symptomen.

De cellulaire samenstelling van het vaatstelsel is min of meer gelijk en als gevolg delen aneurysmata verschillende klinische en genetische risicofactoren. In hoofdstuk 3 is bekeken, in een retrospectieve studie, of zowel extracraniële carotis als intracraniële (binnen de schedel) aneurysmata gelijktijdig voorkomen in een Nederlands cohort van patiënten met een bewezen intracranieel aneurysma. De zogeheten co-prevalentie bleek $1.9 \%$ indien de halsslagaders volledig (van aortaboog tot aan schedeldak) waren afgebeeld. Om deze resultaten te repliceren is in hoofdstuk 4 een soortgelijke studie verricht in een Fins cohort van patiënten met een intracranieel aneurysma, waarbij het volledig afbeelden van de halsslagaders opgenomen is in een standaard beeldvormingsprotocol voor deze patiënten. De co-prevalentie van extracraniële carotis aneurysmata in Finse patiënten met een intracranieel aneurysma blijkt opvallend dubbel zo groot te zijn. In de analyse van zowel Nederlandse en Finse patiënten, waarin gecorrigeerd is voor onderlinge verschillen in patiëntkarakteristieken, blijken het mannelijk geslacht en de Finse nationaliteit de meest voorspellende factoren voor de aanwezigheid van een extracranieel carotis aneurysma. Hoofdstuk 4 toont daarnaast aan dat ten minste $25 \%$ van alle extracraniële carotis aneurysmata wordt gemist, indien de halsslagaders maar gedeeltelijk worden afgebeeld. Dit laatste is het geval in bijvoorbeeld een computer tomografie (CT) scanprotocol brein, waarbij het scanniveau van de kaaklijn tot en met het schedeldak 
bedraagt. Beeldvorming inclusief de volledige arteria carotis interna wordt zodoende voorgesteld als referentie beeldvorming. Concluderend lijkt screenen op extracraniële carotis aneurysmata in patiënten met een intracranieel aneurysma, gezien de lage coprevalentie, niet nodig. De aanwezigheid van beide aneurysmata bij één patiënt, zou echter kunnen duiden op een uniek vaattype.

In hoofdstuk 5 is een systematisch review beschreven van alle, voornamelijk retrospectieve, literatuur die de co-prevalentie van aneurysmata in verschillende slagaders beschrijft. Ongeveer één op de zes patiënten met een primair aneurysma blijkt een tweede aneurysma te hebben, en één uit vier als het een patiënt met een popliteaal aneurysma (slagader in de knieholte) betreft. Naast klassieke vasculaire risicofactoren (hoge leeftijd, hypertensie en atherosclerose), blijken de aanwezigheid van meer dan twee aneurysmata en de grootte van het primaire aneurysma voorspellend voor co-prevalentie van aneurysmata in verschillende vaatstelsels. Toekomstige studies zijn nodig om de ziektelast te bepalen wanneer het bijkomstige aneurysma niet behandeld wordt, alvorens de klinische implementatie uit te voeren van additionele screening in patiënten met een primair aneurysma.

Naast het bepalen van klinische risicofactoren zijn in de afgelopen jaren ook vele genetische studies verricht om de overerfbaarheid, vooral bij patiënten met een abdominaal aorta aneurysma (buikslagader), te begrijpen. Naast bewijs voor individuele genetische varianten die geassocieerd zijn met het voorkomen van aneurysmata, blijken deze complexe multifactoriële ziekten vaak mede veroorzaakt te worden door heel veel verschillende genetische varianten samen (ook wel polygeen). In hoofdstuk 6 zijn de bekende genetische varianten voor aneurysmata van de buikslagader getest voor associatie met klinische eindpunten, zoals aneurysma diameter, aneurysma gerelateerde symptomen (drempelwaarden voor chirurgische behandeling) en arterie type. Voor deze studie zijn gegevens van patiënten geanalyseerd met of een aneurysma gelegen in de buikslagader, arteria poplitea (knieholte), arteria iliaca (lies), arteria femoralis (bovenbeen) of arteria carotis (halsslagader). De polygene risico score, gemodelleerd op basis van eerdere genetische studies, blijkt in deze studie geassocieerd te zijn met de maximale diameter van de aneurysmazak. Zodoende toont hoofdstuk $\mathbf{6}$ de potentiële klinische toepasbaarheid van polygene analyses in patiënten met een aneurysma. Genetische risicostratificatie aanvullend aan klinische risicofactoren kunnen toekomstige individuele behandelingsstrategieën, ofwel personalised medicine, mogelijk maken.

\section{Nieuwe follow-up beeldvormingstechnieken}

Tegenwoordig is beeldvormende software onmisbaar in de behandeling en follow-up van aneurysmata. Het monitoren van de grootte van aneurysma gebeurt door de maximale 


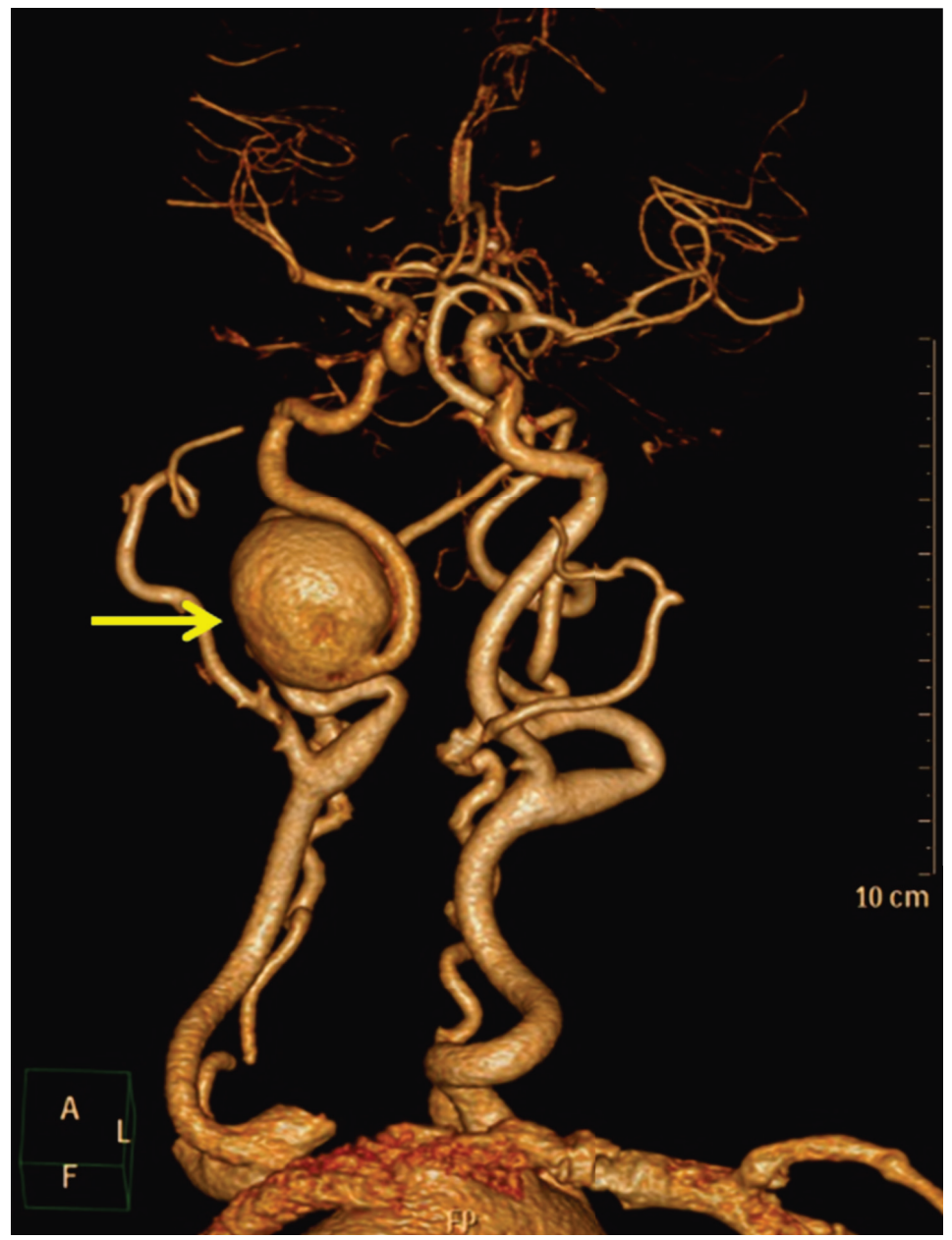

Figuur 1. CTA 3D reconstructie van een patiënt met een extracranieel carotis aneurysma (gele pijl) van de rechter arteria carotis interna. Referentie: Carotis Aneurysma Registratie.

diameter van de aneurysmazak te meten door middel van duplex ultrasonografie, CT of magnetische resonantie (MR) scans. Door de grote verscheidenheid in vorm en grootte van extracraniële carotis aneurysmata is het waarschijnlijk dat de aneurysmazak ook op andere doorsnedes, dan alleen ter plaatse van de maximale diameter, kan groeien. Conventionele maximale diameter metingen worden onvoldoende geacht in het detecteren van dergelijke groei van extracraniële carotis aneurysmata. In hoofdstuk 7 wordt derhalve een protocol voorgesteld op basis van volumemetingen van extracraniële carotis aneurysmata. Het protocol is getest in 40 CT-scans van twintig patiënten met een extracranieel carotis aneurysma, op basis van handsegmentaties, verricht door twee 


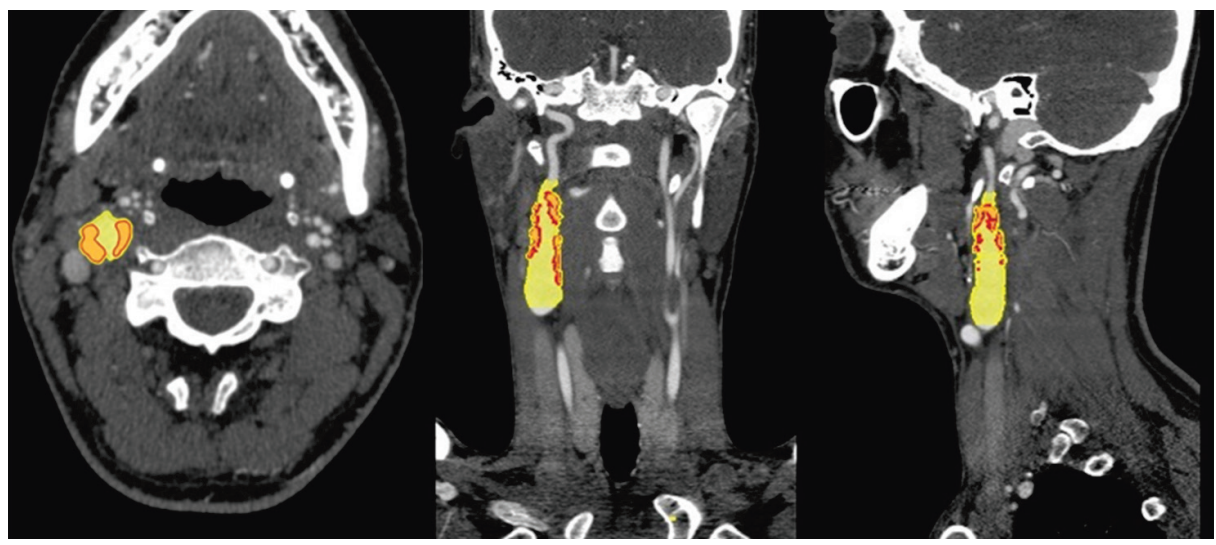

Figuur 2. CT-scan met contrast van een patiënt met een extracranieel carotis aneurysma van de rechter arteria carotis interna, waar door middel van gekleurde handsegmentatie verschillende vaatwand componenten zijn aangegeven. Referentie: Carotis Aneurysma Registratie.

beoordelaars onafhankelijk van elkaar. De reproduceerbaarheid en overeenkomsten van de handsegmentaties blijken uitstekend, en zodoende is deze studie een opstap naar toekomstige vol- of semiautomatische software voor volumebepalingen van extracraniële carotis aneurysmata. In andere vaatbedden, zoals de aorta en intracraniële slagaders, worden met behulp van neurale netwerken en kunstmatige intelligentietechnieken, software ontwikkeld om het aneurysma en de vaatwand beter in kaart te brengen. Naar verwachting zullen deze ontwikkelingen in de toekomst conventionele diameter bepalingen completeren. Potentieel kunnen de gegevens verkregen met deze automatische software, zoals aneurysmavolume, locatie van groei, opbouw en dikte van de vaatwand (Figuur 2), de drempelwaarde voor operatieve behandeling op basis van aneurysma diameter uiteindelijk vervangen.

In hoofdstuk $\mathbf{8}$ is bij vijftien asymptomatische (zonder klachten) patiënten met een extracranieel carotis aneurysma onderzocht of MR beeldvorming met gadolinium (Gd)-contrast van de aneurysmatische vaatwand uitvoerbaar was. Gd-opname in de vaatwand kan duiden op lokale inflammatie en wordt bij intracraniële aneurysmata gesuggereerd als radiologische marker voor groei. De Gd-opname in de vaatwand is onderzocht in relatie tot groei van extracraniële carotis aneurysmata en de geassocieerde cerebrale uitkomst. Alle MR scans zijn door twee beoordelaars onafhankelijk van elkaar gescoord. Bij de nulmeting blijkt dat bijna alle patiënten Gd-opname van de aneurysmatische vaatwand tonen. Bij de controlescan na een jaar, zijn maar vier aneurysmata in diameter toegenomen (twee met en twee zonder Gdopname bij de nulmeting). Hoewel de reproduceerbaarheid van de resultaten in deze studie goed is, zal een groter aanvullend 
onderzoek moeten plaatsvinden om de relatie van Gd-opname met groei van extracraniële carotis aneurysmata te kunnen verhelderen. Een specifiek MR-scanprotocol voor de extracraniële arteria carotis is hierbij cruciaal. Een ander opvallend resultaat is dat deze overwegend jonge en asymptomatische patiëntengroep (mediaan leeftijd 55 jaar) veelal een dubbelzijdig hoge score aan witte stofafwijkingen in het brein hebben in vergelijking met anderszins gezonde leeftijdsgenoten. Witte stofafwijkingen zijn geassocieerd met een verhoogd risico op toekomstige beroertes en cognitieve achteruitgang. De causale relatie tussen extracraniële carotis aneurysmata en witte stofafwijkingen is hiermee niet aangetoond, maar kan duiden op een gemeenschappelijke onderliggende vaataandoening. Cerebrale gevolgen op de langere termijn voor patiënten met een extracranieel carotis aneurysma moeten verder worden bepaald. Daarnaast kan de conservatieve behandeling met medicatie verder geoptimaliseerd worden, door niet alleen te focussen op thromboembolische events vanuit het aneurysma, maar ook om risicofactoren van een onderliggende vaatziekte te behandelen. Idealiter kan Gd-opname in de toekomst gebruikt worden als radiologische marker voor aneurysma instabiliteit en de daarmee geassocieerde cerebrale gevolgen. Zodat enerzijds hoog-risico patiënten geïdentificeerd en invasief behandeld worden en anderzijds conservatief behandelde laag-risico patiënten minder vaak controle beeldvorming moeten ondergaan.

Tot slot beschrijft hoofdstuk 9 een studie waarin arteriële tortuositeit (vele bochten in de slagaders) wordt gemeten met behulp van gespecialiseerde software voor CT angiografie. Arteriële tortuositeit blijkt geassocieerd met dissectie van de halsslagaders, en zoals eerder aangegeven is dissectie een belangrijke oorzaak van extracraniële carotis aneurysmata. Wanneer arteriële tortuositeit ook geassocieerd is met extracraniële carotis aneurysmata, onafhankelijk van arteriële dissectie, kan tortuositeit van de halsslagader mogelijk als screeningsmarker voor aneurysmata gebruikt worden. Zodoende beschrijft hoofdstuk 9 een cross-sectionele case-controle studie waarin bekeken wordt of een hoge carotis tortuositeit geassocieerd is met een extracranieel carotis aneurysma. Patiënten met een carotis dissectie in hun medische voorgeschiedenis zijn uitgesloten van het onderzoek. De studie toont dat carotis tortuositeit binnen de patiënt met een extracranieel carotis aneurysma dubbelzijdig (linker en rechter a. carotis) vrijwel gelijk is, maar onderscheidend hoger is in vergelijking met controle patiënten met een identiek geslacht en leeftijd. Na correctie voor kenmerken die mogelijk invloed hebben op arteriële tortuositeit (zoals leeftijd, geslacht, linker of rechter arteria carotis, hypertensie en de aanwezigheid van een aneurysma), blijkt leeftijd boven de 55 jaar en de aanwezigheid van een extracranieel carotis aneurysma het meest voorspellend voor een verhoogde arteriële tortuositeit. Samenvattend blijkt arteriële tortuositeit geassocieerd met extracraniële carotis aneurysmata en kan idealiter in de toekomst gebruikt worden als screeningsmarker. De invloed van een halsslagader dissectie op deze resultaten kan echter 
niet volledig worden uitgesloten. Het verkrijgen van een compleet dissectie-vrij cohort is uitdagend, doordat de radiologische identificatie van een carotis dissectie bemoeilijkt wordt door het tijdsafhankelijke vasculaire herstel. Desalniettemin onderstreept de studie de hoge tortuositeit bij patiënten met een extracranieel carotis aneurysma, en arteriële tortuositeit kan negatieve gevolgen hebben voor een potentiële endovasculaire behandeling. Aanvullende studies zijn nodig die de kwantitatieve drempelwaarde van carotis tortuositeit aangeven voor een technisch ongecompliceerde endovasculaire behandeling in deze patiëntengroep. Op deze manier worden patiënten met de meest geschikte vasculaire anatomie behandeld met endovasculaire therapie, met mogelijk minder complicaties en re-operaties tot gevolg.

\section{Conclusies}

Aneurysmata van de extracraniële arteria carotis zijn zeldzaam en daardoor is er nog geen eenduidig inzicht in het natuurlijk beloop, de risicofactoren voor ongewenste cerebrale uitkomst, de optimale behandeling en/of follow-up strategie. Toekomstige analyses van de internationale Carotis Aneurysma Registratie gaan hier meer duidelijkheid ingeven. Inzicht in het klinisch risicoprofiel van aneurysmata is nuttig voor het begrijpen van de ziekte in andere vaatbedden. Aanvullende studies zijn echter nodig om de ziektelast te bepalen wanneer het additionele aneurysma, waarop gescreend wordt, onbehandeld blijft. Verdere ontwikkelingen in automatische beeldvormingssoftware zijn nodig om kwantitatief de aneurysma vaatwand en cerebrale uitkomst te monitoren. Klinische, radiologische en genetische risicofactoren kunnen worden ingezet voor de identificatie van patiënten met een extracranieel carotis aneurysma, die een hoog-risico voor aneurysma instabiliteit en geassocieerde cerebrale uitkomst hebben. Risicostratificatie op basis van deze factoren is essentieel in het bepalen van optimale individuele behandelingsstrategie voor patiënten met een extracranieel carotis aneurysma. 



\section{Appendices}

Review Committee Authors and affiliations

List of publications Acknowledgements | Dankwoord About the author 


\section{Review Committee}

Prof. dr. M.L. Bots

Professor of Epidemiology of Cardiovascular Disease, Julius Center for Health Sciences and Primary Care, University Medical Center Utrecht, Utrecht, The Netherlands

Prof. dr. J. Hendrikse

Professor of Radiology, University Medical Center Utrecht, Utrecht, The Netherlands

Prof. dr. G.J.E. Rinkel

Professor of Neurology, University Medical Center Utrecht, Utrecht, The Netherlands

Prof. dr. R.E.G. Schutgens

Professor of Hematology, van Creveldkliniek, University Medical Center Utrecht, Utrecht, The Netherlands

Prof. dr. C.J.A.M. Zeebregts

Professor of Vascular Surgery, University Medical Center Groningen, Groningen, The Netherlands 


\section{Authors and affiliations}

University Medical Center Utrecht, Utrecht, The Netherlands

Department of Vascular Surgery

Prof. Gert J. de Borst, MD

Prof. Dominique P.V. de Kleijn

Constantijn E.V.B. Hazenberg, MD PhD

Joost A. van Herwaarden, MD PhD

Jurre Klaassen, MSc

Vanessa E.C. Pourier, MD PhD

Marjolijn L. Rots, MD PhD

Evelien E. de Vries, MD PhD

Department of Cardiology

Jessica van Setten, MD PhD

Image Sciences Institute

Prof. Max Viergever, MD

Hugo J. Kuijf, $\mathrm{PhD}$

Julius Center for Health Sciences and Primary Care, Epidemiology of Cardiovascular Disease Prof. Michiel L. Bots, MD

Laboratory of Clinical Chemistry and Hematology

Prof. Gerard Pasterkamp, MD

Sander W. van der Laan, PhD

Department of Neurology

Prof. Gabriel J.E. Rinkel, MD

Mervyn D.I. Vergouwen, MD PhD

Department of Radiology

Prof. Jeroen Hendrikse, MD

Prof. Tim Leiner, MD

Department of Traumatology

Quirine M.J. van der Vliet, MD PhD 


\section{Utrecht University, Utrecht, The Netherlands}

Medicine

Nikita K.N. Jorritsma, BSc

\section{Helsinki University Central Hospital, Helsinki, Finland}

Department of Neurology

Prof. Turgut Tatlisumak, MD

Tiina M. Metso, MD PhD

Daniel Strbian, MD PhD

\section{Kuopio University Hospital, Kuopio, Finland}

Department of Neurology and Neurosurgery

Prof. Juha E. Jääskeläinen, MD

Antti E. Lindgren, MD PhD

Mayo Clinic, Rochester, Minnesota, United States of America

Department of Radiology

Waleed Brinjikji, MD PhD

Neurological Institute, University of Pisa, Pisa, Italy

Department of Clinical and Experimental Medicine

Prof. Michelangelo Mancuso, MD

Elena Ferrari, MD PhD

\section{University Hospital Bern, Bern, Switzerland}

Department of Neurology

Prof. Marcel Arnold, MD

Mirjan Heldner, MD PhD

Barbara Simonetti Goeggel, MD PhD

Valentin K. Steinsiepe, MD

Institute of Diagnostic and Interventional Neuroradiology

Prof. Jan Gralla, MD

University Hospital Moscow, Moscow, Russia

Research Center of Neurology

Prof. Ludmilla Kalashnikova, MD

Maria Danilova, MD PhD

Marina Dreval, MD PhD 


\section{Uppsala University, Uppsala, Sweden}

Department of Surgical Sciences, Vascular Surgery

Prof. Martin Björck, MD

Virginia Commonwealth University Medical Center, Richmond, Virginia, United States of America

Department of Emergency Medicine

Jessica Balderston, MD PhD 


\section{List of publications}

Timmerman N, Rots M, van Koeverden I, Haitjema S, van Laarhoven CJHCM, Vuurens AM, den Ruijter HM, Pasterkamp G, Kappelle LJ, de Kleijn DPV, de Borst GJ. Cerebral small vessel disease in standard preoperative imaging reports is independently associated with poor survival following carotid endarterectomy. Eur J Vasc Endovasc Surg 2020; In press.

van Laarhoven CJHCM*, Rots $\mathrm{ML}^{\star}$, Pourier VEC, Jorritsma NKN, Leiner T, Hendrikse J, Vergouwen MDI, de Borst GJ. Gadolinium enhancement of the aneurysm wall in extracranial carotid artery aneurysms. AJNR 2020;41:501-507.

van Laarhoven CJHCM, Pourier VEC, Lindgren AE, Vergouwen MDI, Jääskelaïnen JE, Rinkel GJE, de Kleijn DPV, de Borst GJ. Co-prevalence of extracranial carotid aneurysms differs between European intracranial aneurysm cohorts. PLoS One 2020,15:e0228041.

van Laarhoven CJHCM, van Setten J, van Herwaarden JA, Pasterkamp G, de Kleijn DPV, de Borst GJ, van der Laan SW. Polygenic susceptibility of aortic aneurysms associates to the diameter of the aneurysm sac: the Aneurysm-Express biobank cohort. Sci Rep 2019;27:19844.

Wallis de Vries BM, Timmerman N, van Laarhoven CJHCM, Visser L, Pol RA, El Moumni M, Pasterkamp G, de Borst GJ, Zeebregts CJ. The effect of metabolic syndrome on the occurrence of restenosis after carotid endarterectomy. Eur J Vasc Endovasc Surg 2019;58:805-812.

van Laarhoven CJHCM${ }^{\star}$, de Vries EE*, Kuijf HJ, Hazenberg CEVB, van Herwaarden JA, Viergever MA, de Borst GJ. Volumetric assessment of extracranial carotid artery aneurysms. Sci Rep 2019;30:8108.

de Vries EE, Pourier VEC, van Laarhoven CJHCM, Vonken EJ, van Herwaarden JA, de Borst GJ. Comparability of semiautomatic tortuosity measurements in the carotid artery. Neuroradiology 2019;61:147-153.

Pourier VEC, van Laarhoven CJHCM, Vergouwen MDI, Rinkel GJE, de Borst GJ. Prevalence of extracranial carotid artery aneurysms in patients with an intracranial aneurysm. PLoS One 2017;12:e187479.

van Laarhoven JJEM, van Lammeren GW, Houwert RM, van Laarhoven CJHCM, Hietbrink F, Leenen LP, Verleisdonk EJ. Isolated hip fracture care in an inclusive trauma system: a trauma system wide evaluation. Injury 2015;46:1042-6 


\section{About the author}

Constantia Josepha Henrina Christina Maria (Constance) van Laarhoven was born on the $24^{\text {th }}$ of September 1991 in Tilburg, The Netherlands, as youngest daughter of Rien and José. Together with three sisters and many farm animals, Constance grew up in the southern Brabant region. After graduating from Theresialyceum Tilburg in 2009 and Luzac College Breda in 2010 (cum laude), she started her medical study at Utrecht University, The Netherlands. During the medical curriculum, her surgical interests were emphasized and this resulted in the start of a scientific career at the Vascular Surgery department of the University Medical Center Utrecht under supervision of prof. dr. G.J. de Borst. After working as a research graduate student at the Neurosurgery department of the Kuopio University Hospital in Kuopio, Finland (prof. dr. J.E. Jääskelainen and dr. A.E. Lindgren), and Neurology department of the University Hospital Bern in Bern, Switzerland (prof. dr. M. Arnold), she obtained her medical degree and started with her $\mathrm{PhD}$ focused on extracranial carotid artery aneurysms in September 2017 (prof. dr. G.J. de Borst and prof. dr. D.P.V. de Kleijn). The results of her studies are presented in this thesis and were discussed at several international conferences within the neurovascular field. In August 2020 she will start her clinical career as a surgical resident not in training (ANIOS) in the Meander Medical Center, Amersfoort, The Netherlands, under supervision of prof. dr. E.C.J. Consten and dr. V. van Weel.

Constance aspires to become a surgical specialist while maintaining her passion for clinical research, sports, and spending time with friends and family. 



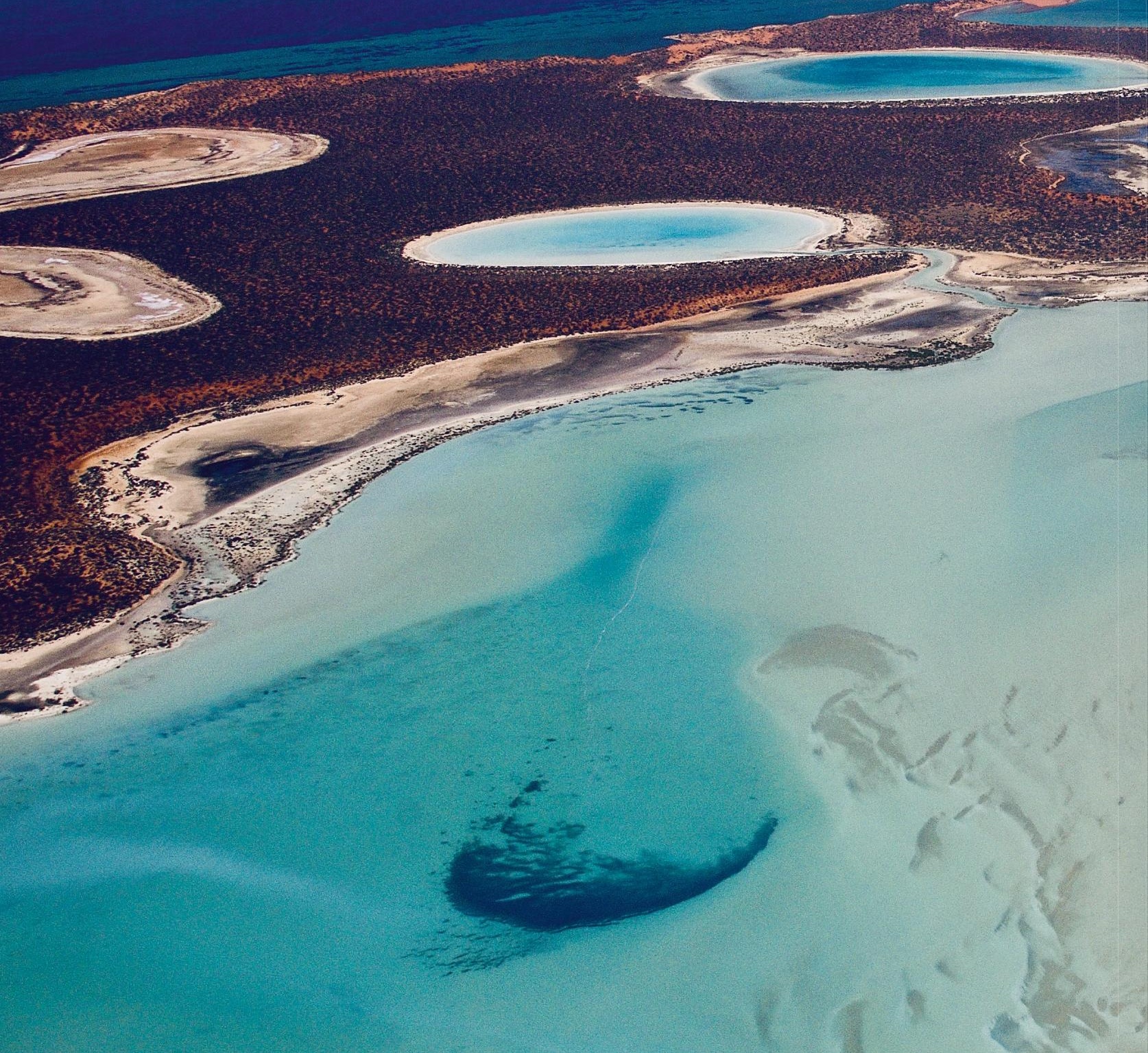

